The Effect of Sugar on the Processing Behaviour of Palm Oil:

From Confectionery Fundamentals to Predictive Regression Models

by

\begin{abstract}
Ryan West
M.Sc., University of Guelph, Guelph, Canada, 2012

M.Sc., Brock University, St. Catharines, Canada, 2009

B.Sc., Brock University, St. Catharines, Canada, 2007
\end{abstract}

A dissertation presented to Ryerson University

in partial fulfillment of the

requirements for the degree of

Doctor of Philosophy

in the program of

Molecular Science

Toronto, Ontario, Canada

(C) Ryan West, 2018 


\section{Author's Declaration}

I hereby declare that I am the sole author of this dissertation. This is a true copy of the dissertation, including any required final revisions, as accepted by my examiners.

I authorize Ryerson University to lend this dissertation to other institutions or individuals for scholarly research.

I further authorize Ryerson University to reproduce this dissertation by photocopying or by other means, in total or in part, at the request of other institutions or individuals for scholarly research.

I understand that my dissertation may be made electronically available to the public. 


\title{
The Effect of Sugar on the Processing Behaviour of Palm Oil: \\ From Confectionery Fundamentals to Predictive Regression Models
}

\author{
Ryan West \\ Ryerson University \\ Doctor of Philosophy \\ Molecular Science
}

(C)2018

\begin{abstract}
This dissertation explores the impact of confectioner's sugar and processing on key physical characteristics of palm oil. Though bulk oils such as palm oil have been well-studied, fatcontinuous dispersions that include other ingredients such as sugar, cocoa solids, or milk powder are much more complex due to ingredient interactions and the resulting changes in properties such as fat crystal morphology and crystallization pathway. These implications restrict the ability to extrapolate responses from bulk oils towards multi-ingredient systems. This is particularly important given that palm oil in foods rarely exists in bulk but is usually mixed with multiple ingredients.
\end{abstract}

The effects of processing on both bulk oil and oil-sugar blends were explored over four weeks of storage and clearly demonstrated that the existence of a dispersion resulted in large differences in rheology and texture compared to the bulk. Adding sugar significantly increased storage modulus and firmness of the oils while exhibiting increased sensitivity towards processing conditions. Predictive models were generated through multiple regression analysis that overcame limitations derived from extrapolation and corrected for 
these behavioural differences using a single binary variable accounting for the presence of confectioner's sugar. As a result, the contributions of sugar to the rheology and texture of oil-sugar blends were modelled, a first in such systems.

The results contained within this dissertation are of great industrial relevance as they:

i) limit the dependence on anecdote and empiricism to explain results,

ii) generate detailed process maps,

iii) identify optimal process values to attain desirable rheological and textural responses, and

iv) open the door for further investigation into dispersion effects. 


\section{Acknowledgements}

There are many people I must acknowledge who have helped me in some way during my time at Ryerson University. I must first thank my advisor, Professor Dérick Rousseau, for his insight and guidance throughout the years. I would also like to thank Professors Darrick Heyd and Stephen Wylie for their contributions as members of my supervisory committee.

I have had the pleasure of working with an amazing research group spanning four years. Particular thanks go to Nicole Green, Ruby Rafanan, and Tu Tran who showed me the ropes when I first joined the lab. Our frequent cheese breaks and one-way coffees have helped in more ways than I initially realized.

I must also thank my large team of undergraduate helpers, without whom would have resulted in a much lengthier project. These people include — in alphabetical order - Adrian Argudo, Iselina Cascone, Majd Ghadban, Kristy Halnan, Veronica Hislop, Nick Jamkhou, Anna Okolnikova, Vera Vece, and Kyle Vollett.

Finally, I must acknowledge my gratitude towards both the Natural Sciences and Engineering Research Council of Canada and the Professional Manufacturing Confectioners' Association for making this research possible through their financial support. 
To Harvey 


\section{Table of Contents}

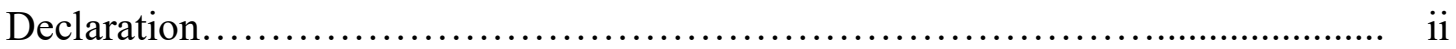

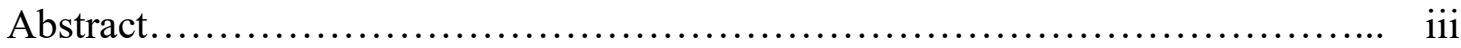

List of Nomenclature........................................................

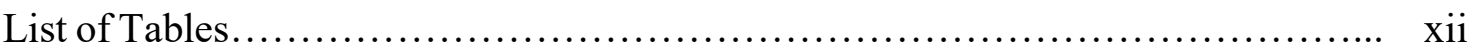

List of Figures......................................................... xiii

List of Appendices.................................................. XV

General Overview...................................................... 1

Chapter 1: The Role of Non-Fat Ingredients on Fat Crystallization................ 4

1.1 Fat-Based Confections............................................. 5

1.1.1 Main Components......................................... 5

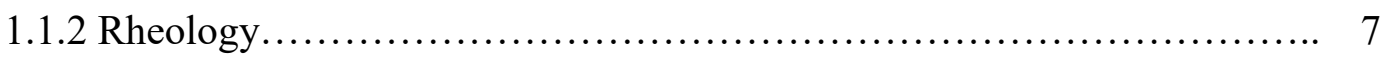

1.2 Fats and Oils....................................................... 13

1.2.1 Molecular Structure .............................................. 13

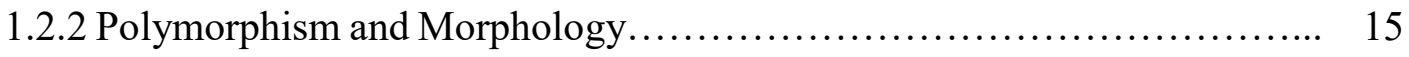

1.2.3 Crystallization............................................... 19

1.2.4 Compositional and Processing Effects............................... 26

1.3 Palm Oil in Application......................................... 28

1.3.1 Production and Use........................................... 29

1.3.2 Composition................................................ 30

1.3.3 Processing.................................................. 32

1.3.4 Recrystallization............................................ 34

1.4 Characterization of Particle Interactions in Confections.................. 39

1.4.1 Force Spectroscopy....................................... 40

1.4.2 Cantilever Functionalization................................... 44

1.5 Conclusions..................................................... 45 
Chapter 2: The Effect of Sugar on the Physical Properties of Palm Oil.

2.1 Introduction.................................................... 48

2.2 Materials and Methods........................................... 50

2.2.1 Sample Preparation............................................ 50

2.2.2 Compositional Analysis...................................... 52

2.2.3 Pulse Nuclear Magnetic Resonance.............................. 52

2.2.4 Differential Scanning Calorimetry............................... 53

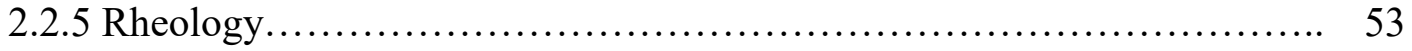

2.2.6 Microscopy............................................... 54

2.2.7 Statistical Analysis.......................................... 54

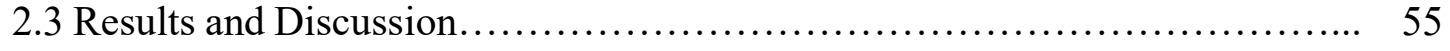

2.3.1 Solid Fat Content and Total Enthalpy............................ 55

2.3 .2 Viscoelasticity........................................... 58

2.3.3 Fat Crystal Morphology......................................... 61

2.3.4 Composition..................................................... 64

2.3.5 Mechanistic Considerations....................................... 65

2.4 Conclusions................................................... 70

Chapter 3: The Effect of Sugar on Response towards Cooling Endpoint in Palm Oil.. 71

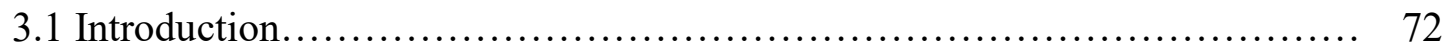

3.2 Materials and Methods........................................... 73

3.2.1 Sample Preparation......................................... 73

3.2.2 Pulsed Nuclear Magnetic Resonance.............................. 75

3.2.3 Differential Scanning Calorimetry.............................. 75

3.2 .4 Microscopy ............................................. 75

3.2 .5 Rheology................................................ 76

3.2.6 Texture Analysis............................................... 76

3.2.7 Statistical Analysis and Multiple Regression........................ 77

3.3 Results and Discussion......................................... 78

3.3.1 Solid Fat Content and Polymorphism............................... 78

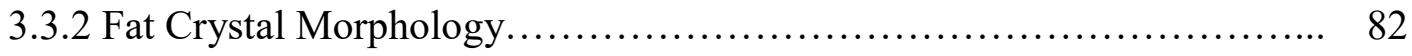




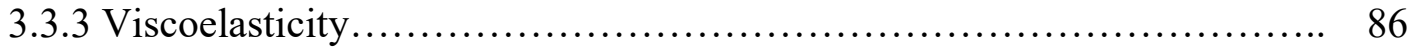

3.3.4 Firmness ............................................................. 90

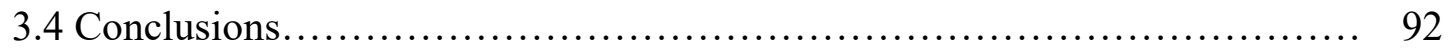

Chapter 4: The Effect of Sugar on Processing Interactions and Optical Process Values in Palm Oil................................................................. 93

4.1 Introduction............................................................. 94

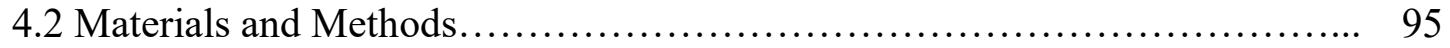

4.2.1 Sample Preparation................................................ 95

4.2.2 Differential Scanning Calorimetry.................................. 96

4.2.3 Microscopy ........................................................ 96

4.2.4 Rheology ........................................................... 97

4.2.5 Texture Analysis..................................................... 97

4.2.6 Statistical Analysis and Multiple Regression........................... 97

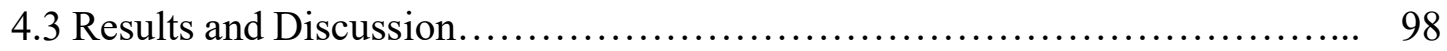

4.3.1 Total Enthalpy and Polymorphism.................................. 98

4.3.2 Storage Modulus and Fat Crystal Morphology............................ 103

4.3.3 Firmness....................................................... 111

4.3.4 Further Considerations with Multiple Regression...................... 115

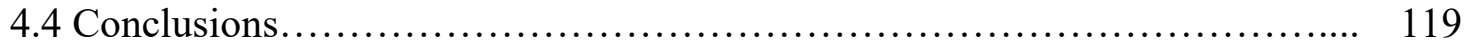

Chapter 5: General Conclusions and Future Work............................ 121

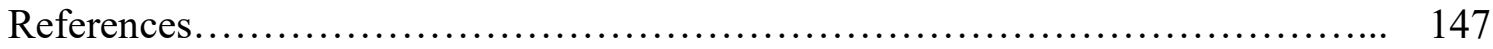




\section{List of Nomenclature}

$\delta$ Phase angle

$\zeta$ Confectioner's sugar

$\eta$ Apparent viscosity

$\eta *$ Complex viscosity

$\boldsymbol{\eta}_{\boldsymbol{\mu}}$ Micro-viscosity

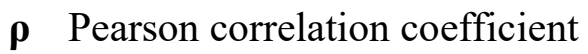

$\tau_{0} \quad$ Yield stress

( Angular frequency

ANOVA Analysis of variance

AFM Atomic force microscopy

$C_{P}$ Mallows' statistic

CB Cocoa butter

CLSM Confocal laser scanning microscopy

CO Canola oil

CVD Cardiovascular disease

DAG Diacylglycerol

DSC Differential scanning calorimetry

F Firmness

FA Fatty acid

FFA Free fatty acid

FID Free induction decay

$\mathbf{G}^{\prime}$ Storage modulus

$\mathbf{G}^{\prime \prime}$ Loss modulus

$\left|\mathbf{G}^{*}\right|$ Complex modulus

$\Delta \mathbf{H}_{\mathbf{T}}$ Total enthalpy of melting

MAG Monoacylglycerol

MF Milkfat

NMR Nuclear magnetic resonance

PCA Principal component analysis 


$\begin{aligned} \text { PGPR } & \text { Polyglycerol polyricinoleate } \\ \text { PHO } & \text { Partially hydrogenated oil } \\ \text { PLM } & \text { Polarized light microscopy } \\ \text { PMF } & \text { Palm mid-fraction } \\ \text { PO } & \text { Palm oil } \\ \boldsymbol{q} & \text { Cooling rate } \\ \boldsymbol{q}_{\boldsymbol{0}} & \text { Optimal cooling rate } \\ \text { SFC } & \text { Solid fat content } \\ \boldsymbol{t} & \text { Storage time } \\ \boldsymbol{\Delta T} & \text { Cooling endpoint deviation } \\ \boldsymbol{\Delta T} \boldsymbol{T}_{\boldsymbol{0}} & \text { Optimal cooling endpoint deviation } \\ \text { TAG } & \text { Triacylglycerol } \\ \text { XRD } & \text { X-ray diffraction }\end{aligned}$




\section{List of Tables}

Table 1.1 Effect of soy lecithin concentration on $\tau_{0}$ and $\eta$ of milk chocolate....... 10

Table 1.2 Effect of CB origin on TAG composition......................... 15

Table 1.3 Melting point and chain-length packing of CB polymorphs.............. 18

Table 1.4 Typical FA composition of PO................................ 31

Table 1.5 DAG and TAG composition of PO............................ 32

Table 1.6 Physical properties of PO and its fractions....................... 34

Table 1.7 Effect of temperature cycles on fat bloom formation................... 37

Table 2.1 Acylglycerol composition of PO, PMF, and control.................. 56

Table 3.1 Effects of sugar and endpoint on $f_{\beta^{\prime}}$ for PO and PMF over time.......... 80

Table 4.1 Optimal process values and associated maximum response at week $4 \ldots \ldots 102$ 


\section{List of Figures}

Figure 1.1 Multiscale interpretation of chocolate from acylglycerol assembly to fat bloom

Figure 1.2 Molecular structure of 1,3-dipalmitoyl-2-oleoylglycerol, 1-palmitoyl-

2,3-dioleoylglycerol, and 1-palmitoyl-2-oleoyl-3-linoleoylglycerol.

Figure 1.3 Polymorphic forms of TAGs

Figure 1.4 Diffractograms of different tristearin polymorphs.

Figure 1.5 Timeline of events during fat crystallization and storage.............. 20

Figure 1.6 Effect of sugar volume fraction on PO crystallization during cooling..... 24

Figure 1.7 Effect of additive on lamellar orientation of LLL................... 25

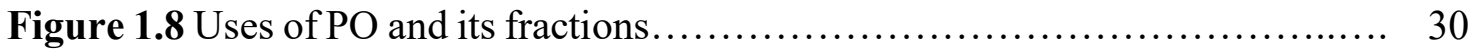

Figure 1.9 Schematic diagram of AFM................................... 41

Figure 1.10 Interpretation of force-distance curves............................ 42

Figure 1.11 Dimension-dependent conformational analysis of a protein depicting the unfolding of conformation II.......................................... 45

Figure 2.1 Melting profiles of bulk PO, PMF, and control..................... 51

Figure 2.2 Effect of sugar on SFC of PO and PMF over time................... 55

Figure 2.3 Effect of sugar on $\Delta \mathrm{H}_{\mathrm{T}}$ of $\mathrm{PO}$ and $\mathrm{PMF}$ over time.................... 57

Figure 2.4 Effect of sugar on $\left|\mathrm{G}^{*}\right|$ and $\delta$ of PO and PMF immediately after processing.

Figure 2.5 Effect of sugar on $\mathrm{G}^{\prime}$ of $\mathrm{PO}$ and $\mathrm{PMF}$ over time..................... 60

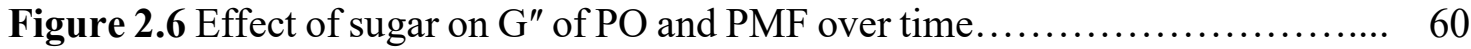

Figure 2.7 Fat crystal morphology of bulk PO and PMF over time............... 62

Figure 2.8 Fat crystal morphology of PO- and PMF-sugar blends over time.......... 63

Figure 2.9 PCA biplot of 'continuous phase' vs. 'dispersion' ...................... 65

Figure 2.10 Effect of sugar on $\eta$ * of control over time....................... 69

Figure 3.1 Effects of sugar and endpoint on SFC of PO and PMF over time.......... 79

Figure 3.2 Effects of sugar and endpoint on PO crystal morphology at week $0 \ldots \ldots \ldots .83$

Figure 3.3 Effects of sugar and endpoint on PMF crystal morphology at week $0 \ldots \ldots \quad 83$

Figure 3.4 Effects of sugar and endpoint on PO crystal morphology at week $4 \ldots \ldots \ldots .85$ 
Figure 3.5 Effects of sugar and endpoint on PMF crystal morphology at week 4.... 85

Figure 3.6 Effects of sugar and endpoint on $\mathrm{G}^{\prime}$ of $\mathrm{PO}$ and $\mathrm{PMF}$ over time............ 87

Figure 3.7 Effects of sugar and endpoint on $\mathrm{G}^{\prime \prime}$ of $\mathrm{PO}$ and PMF over time.......... 87

Figure 3.8 Effects of sugar and endpoint on F of PO and PMF over time........... 91

Figure 4.1 Effects of sugar and processing conditions on $\Delta \mathrm{H}_{\mathrm{T}}$ of $\mathrm{PO}$ and $\mathrm{PMF}$ over time.

Figure 4.2 Impact of sugar and endpoint on polymorphic composition of PO and PMF cooled at $10^{\circ} \mathrm{C} \cdot \mathrm{min}^{-1}$

Figure 4.3 Predicted process maps of $\Delta \mathrm{H}_{\mathrm{T}}$ according to models $4.1 \mathrm{a}$ and $4.1 \mathrm{~b} .$.

Figure 4.4 Effects of sugar and processing conditions on $\mathrm{G}^{\prime}$ of PO and PMF over time.

Figure 4.5 Effects of sugar and storage on PO crystal morphology processed under standard conditions

Figure 4.6 Effects of sugar and cooling rate on final PO crystal morphology (e.g., week 4) cooled to $20^{\circ} \mathrm{C}$.

Figure 4.7 Effects of processing on initial crystal morphology (e.g., week 0) of POsugar blends.

Figure 4.8 Predicted process maps of $\mathrm{G}^{\prime}$ according to models $4.2 \mathrm{a}$ and $4.2 \mathrm{~b}$.

Figure 4.9 Effects of sugar and processing conditions on F of PO and PMF over time.

Figure 4.10 Predicted process maps of $F$ according to models $4.3 \mathrm{a}$ and $4.3 \mathrm{~b}$.

Figure 4.11 Effect of sugar and endpoint on the correlation between small and large deformation tests.

Figure 4.12 Comparison between observed and predicted responses from bulk (extrapolated) and full datasets.

Figure 4.13 Comparison between maximum response of oil-sugar blends against response predicted from process values optimized for bulk systems 


\section{List of Appendices}

A1 Frequency Sweep Tests............................................ 123

A2 ANOVA Tables.................................................... 128

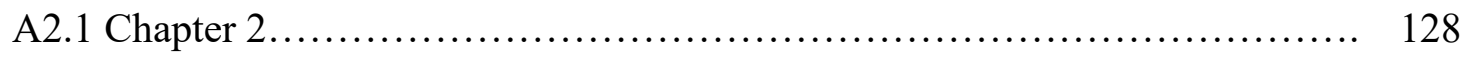

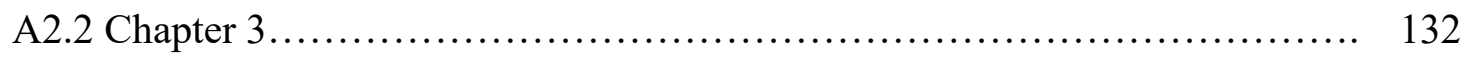

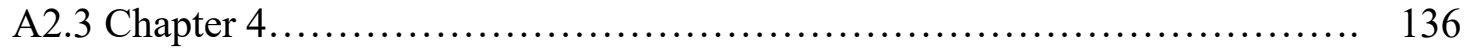

A3 Multiple Regression Analysis........................................ 139

A3.1 Sample Calculation............................................. 139

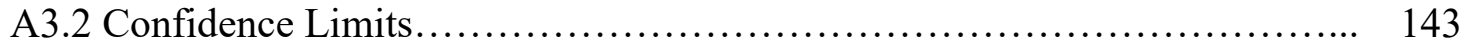

A4 Crystal Morphology of Palm Mid-Fraction.............................. 146 


\section{Introduction}

\section{General Overview}

Confectionery fillings and coatings are complex materials consisting of multiple interacting ingredients. Though their production methods have been known for decades, there remains a dearth of knowledge regarding how sugar dispersions impact fat crystallization in such materials. To this day, many opportunities to improve process efficiency while maintaining shelf life and consumer acceptability of such products exist. The content within this dissertation is exceedingly relevant to the confectionery industry as it pertains to process optimization in confectionery product lines and product quality and stability.

Chapter 1 of this dissertation reviews the role that non-fat ingredients have on fat crystallization with an emphasis on confectionery applications. This is followed by the establishment of a baseline effect that confectioner's sugar has on the physical properties of palm oil processed under standard conditions (Chapter 2). From there, the effect that sugar has on processing (Chapter 3) as well as processing interactions (Chapter 4) are explored. In this last chapter, multiple regression analysis is utilized to create process maps and determine optimal process values that yield maximum predicted response of these physical properties.

\section{Dissertation Objectives}

The overall objective of this dissertation is to understand the effect that confectioner's sugar has on palm oil crystallization with the goal of providing predictive tools to reduce saturated fat content and improve production efficiency. The specific research objectives were: 
i. to establish a baseline effect that confectioner's sugar has on palm oil crystallization processed under standard conditions (Chapter 2),

ii. to determine how confectioner's sugar alters the response of palm oil towards cooling endpoint temperature while introducing the concept of multiple regression analysis (Chapter 3), and

iii. to generate process maps and optimal process values from predictive models that correct for the effect of confectioner's sugar on processing interactions (Chapter 4).

\section{Hypotheses}

The proposed hypotheses were that:

i. the addition of confectioner's sugar would facilitate fat crystallization, thereby increasing viscoelasticity and textural properties,

ii. higher cooling rates from the melt would enhance crystallization (rate and extent), storage modulus, and firmness while reducing crystal size and polymorphic stability,

iii. higher endpoint temperatures would restrict crystallization as processing ends prematurely, ultimately reducing storage modulus and firmness, and

iv. the general presence of a dispersed phase would alter processing response in palm oil limiting the ability to predict trends in oil-sugar blends based on the behaviour of bulk systems without the aid of statistical modelling.

\section{Contribution of Authors}

All experimental work enclosed within this dissertation was performed by myself. Furthermore, I am the primary author of all publications produced from this dissertation. These publications include: 
i. West, R. and Rousseau, D. (2018). The role of non-fat ingredients on confectionery fat crystallization. Crit. Rev. Food Sci. Nutr. 58(11):1917-1936 (Chapter 1).

ii. West, R. and Rousseau, D. (2016). Crystallization and rheology of palm oil in the presence of sugar. Food Res. Int. 85:224-234 (Chapter 2).

iii. West, R. and Rousseau, D. (2017). Modelling sugar, processing, and storage effects on palm oil crystallization and rheology. LWT - Food Sci. Technol. 83:201-212 (Chapter 3).

iv. West, R. and Rousseau, D. (2018). Regression modelling of the impact of sugar and temperature on palm oil crystallization and rheology. Food Chem. (under review, Chapter 4). 


\section{Chapter 1:1.1}

\section{THE ROLE OF NON-FAT INGREDIENTS ON FAT CRYSTALLIZATION}

Confections such as chocolate and biscuit fillings are composed of a continuous fat phase that contains dispersed non-fat ingredients such as sugar and cocoa powder. Research on fat crystallization and rheology in confections often extrapolates crystallization and textural properties from bulk to mixed systems while overlooking the important role of composition or particle interactions. For example, in chocolate processing the fat phase aids dispersed phase lubrication and fluidity whereas the dispersed particles assist in fat crystallization by providing many nucleation sites. In confections with a high dispersed phase volume fraction, fat crystallization may be hindered due to reduced triacylglycerol mobility, confinement, and increased tortuosity (i.e., twisting degree within a porous medium). This is further complicated in systems with slow crystallizing fats such as palm oil whose crystallization is exceptionally sensitive to composition and processing. This chapter breaks down the physical chemistry of fat-based confections and discusses the impact of different non-fat ingredients towards fat crystallization and rheology. The behaviour of palm oil is further highlighted as it is becoming increasingly popular as a confectionery ingredient. Lastly, ingredient-ingredient interactions and their role in fat crystallization are described along with force spectroscopy as a novel tool to characterize such phenomena. Force spectroscopy utilizes atomic force microscopy to measure intermolecular forces as a function of distance but remains largely unexplored in food science.

\footnotetext{
1.1 This chapter previously appeared as a published review article with following citation: West, R. and Rousseau, D. (2018). The role of non-fat ingredients on confectionery fat crystallization. Crit. Rev. Food Sci. Nutr. 58(11):1917-1936.
} 


\subsection{Fat-Based Confections}

\subsubsection{Main Components}

Processed foods are composed of numerous ingredients often existing in a multi-phasic environment with structures ranging from the nano- to the macro-scale (Walstra, 2003; Rao, 2007). In fat-based confections, non-fat ingredients such as sugar, salts, cocoa solids, and/or proteins are dispersed within a continuous semi-solid fat phase (Götz et al., 2005; Sokmen and Gunes, 2006; Afoakwa et al., 2009; Dahlenborg et al., 2011; Carvalho-da-Silva et al., 2013; Svanberg et al., 2013), whose fat crystal morphology, polymorphic behaviour, and interactions with non-fat ingredients determine processability, quality, and sensory properties of the final product (Figure 1.1). Chocolate, which is perhaps the most popular and well-studied of these confections, is solid-like at room temperature (e.g., $\left.\leq 25^{\circ} \mathrm{C}\right)$, but melts rapidly once in the mouth (e.g., 33-38 ${ }^{\circ} \mathrm{C}$ ) (Afoakwa et al., 2008a). The proper crystallization of cocoa butter (CB), which can account for up to $30-40 \%(\mathrm{w} / \mathrm{w})$ of the product, is largely responsible for the sensory behaviour that consumers have come to expect (Afoakwa et al., 2007; Rousseau, 2007; Fernandes et al., 2013).

Often, the main ingredient of fat-based confections is sucrose (Krüger, 1999; Beckett, 2000), which provides sweetness and mouthfeel (Jamieson, 2008) as well as energy $\left(4 \mathrm{kcal} \cdot \mathrm{g}^{-1}\right)$ (Aidoo et al., 2013). Adjusting the sugar content by as little as $5 \%$ (w/w) can significantly impact the perceived sweetness in some food applications (Beckett, 2008). In products such as chocolate, optimal sugar particle size is approximately $30 \mu \mathrm{m}$ in diameter to maintain proper textural properties (Jeffery, 1993). Coarser particles tend to confer grittiness while finer particles impact fluidity (Beckett, 2000). A particle size distribution of $60 \%$ coarse 


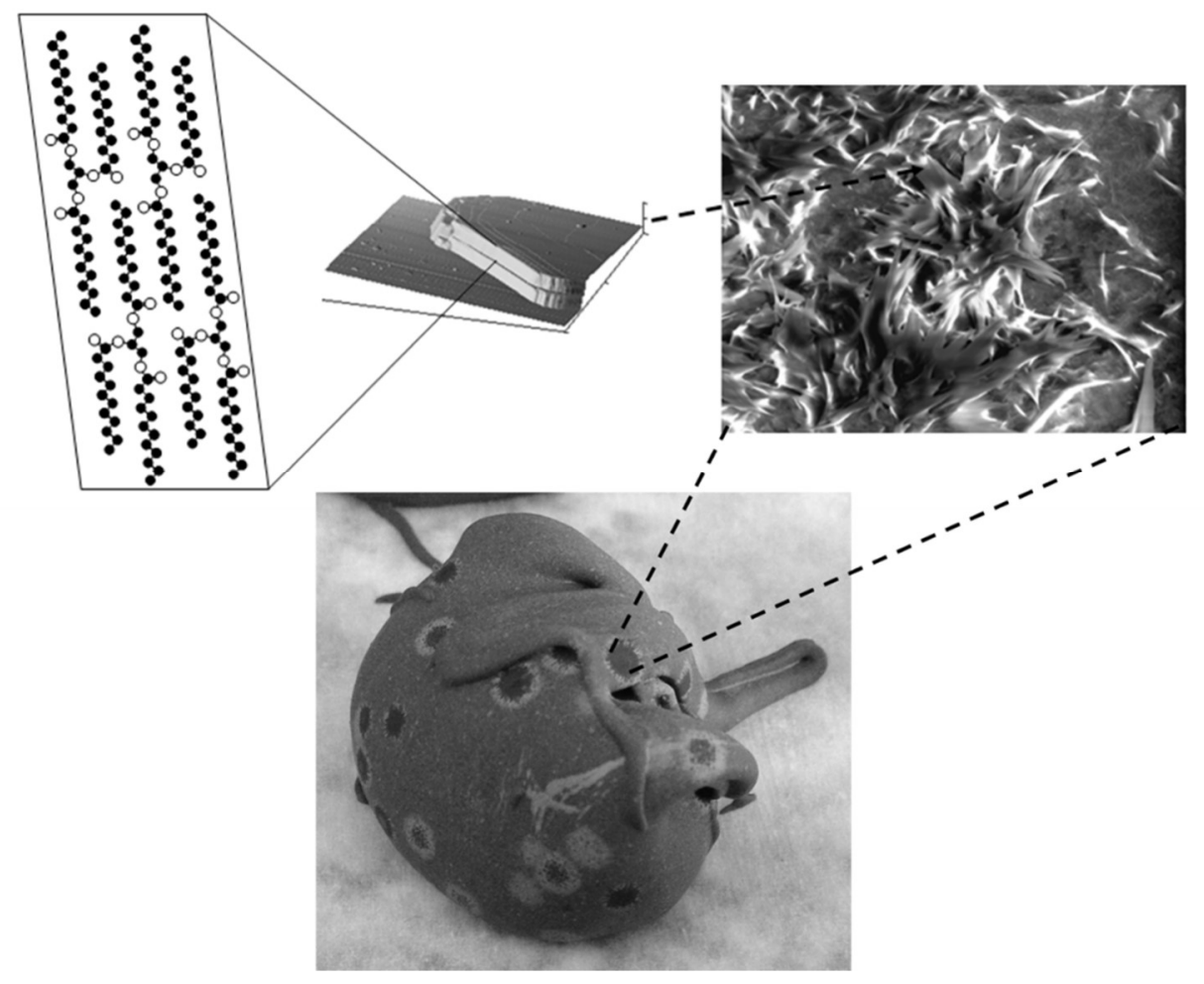

Figure 1.1 Multiscale interpretation of chocolate from acylglycerol assembly to fat bloom.

and $40 \%$ fine particles has been shown to minimize the relative viscosity of fluid chocolate (Servais et al., 2002).

Emulsifiers are added to confections to lubricate and aid in the dispersion of sugar and other non-fat ingredients within fat-continuous environments (Beckett, 2000; Götz et al., 2005; Afoakwa et al., 2007; Van Der Vaart et al., 2013). The most commonly used emulsifiers are soy lecithin, which contains phospholipids that contribute to its amphiphilic properties, and polyglycerol polyricinoleate (PGPR), a synthetic equivalent that shows a greater reduction in confectionery yield stress $\left(\tau_{0}\right)$ than soy lecithin (Vernier, 1998; Schantz et al., 2003). Other non-fat ingredients commonplace in confections includes milk solids, composed of casein and whey proteins, which have traditionally been added to provide a 'creamy' 
mouthfeel (Haylock and Dodds, 1999), and cocoa solids, which contribute to flavour, texture, and are a source of antioxidants (Doká et al., 2013).

\subsubsection{Rheology}

This section summarizes the role of key ingredients found in confections on rheology, including the fat phase, sucrose, milk proteins, cocoa powder, and emulsifiers. The rheological properties of foods are important given their close association with processing efficiency, e.g., extrusion, enrobing, etc., and organoleptic properties (Kilcast and Clegg, 2002; Servais et al., 2002, 2004; Engelen et al., 2005; Ahmed and Ramaswamy, 2006; Denker et al., 2006; Baixauli et al., 2007; Engelen and Van Der Bilt, 2008; Ziegleder, 2009; Liu et al., 2010; Zarić et al., 2012). Oscillatory rheology is used to explore confectionery fat crystallization in the absence of shear, i.e., under conditions where the material is not irreversibly deformed (Omar et al., 2005; De Graef et al., 2006). By contrast, rotational rheology explores the influence of both torque and rotational shear on material properties, such as the apparent viscosity $(\eta)$ of dark chocolate during conching (Vivar-Vera et al., 2008).

The rheological properties of confections depend on the physical properties of the continuous fat phase, e.g., solid fat content (SFC), crystal morphology, etc., the volume fraction of non-fat ingredients, and specific ingredient interactions (Tscheuschner and Wünsche, 1979; Van Den Tempel, 1979; Jeffery, 1993; Tscheuschner, 1994; Bouzas and Brown, 1995; Kulozik et al., 2003; Liang and Hartel, 2004; Schantz and Rohm, 2005; Sokmen and Gunes, 2006; Do et al., 2007; Pérez-Martínez et al., 2007; Rao, 2007; Tang and Marangoni, 2007). 
Fat-based confections are often shear-thinning, non-Newtonian pseudoplastic materials, possessing a measurable $\tau_{0}$ (Chevalley, 1999; Rector, 2000; Schantz and Rohm, 2005; Chhabra, 2006). Reduction of the continuous phase volume, as seen in fat-reduced products, results in a harder, more brittle texture (Beckett, 1999; Spearing, 2007). In fat-reduced dark chocolate, $\eta$ has been shown to increase because of the reduced lubrication of sugar and other non-fat ingredients (Afoakwa et al., 2008b). In contrast, the addition of the continuous phase results in a smoother, more fluid product (Aidoo et al., 2012) and plateaus at $32 \%$ (w/w), above which no continued effect is seen (Afoakwa et al., 2007). The proportion of fat used in confections must also be considered during processing because of its impact on pumping, moulding, and other unit operations (Do et al., 2008). For example, chocolate may be pumped within pipelines at a flow rate of $10 \mathrm{t} \cdot \mathrm{h}^{-1}$ across several dozens of metres within a production setting (Walker, 2009). Complications due to fat-reduced formulations, such as increased hardness and difficulty to swallow (Beckett, 1999; Spearing, 2007), have been circumvented by either increasing emulsifier content or the addition of $\mathrm{CB}$ replacers (Wang et al., 2010), oleogels (Kaiser et al., 1998; Marangoni, 2012), or limonene (Beckett, 1999; Do et al., 2008), all of which help to maintain proper rheology.

Both volume fraction and size distribution of a dispersion strongly impact rheology. Importantly, surface area and interactions with the continuous phase increase with a reduction in particulate size as this increases the energy barrier needed to overcome $\tau_{0}$ and results in a much higher $\eta$ (Beckett, 1994; Mongia and Ziegler, 2000; Ziegler et al., 2001; Walstra, 2003; De Graef et al., 2011; Fernandes et al., 2013). For the consumer, this is perceived as increased hardness and decreased spreadability (Chevalley, 1999; Beckett, 2000; Afoakwa et al., 2008b, 2008c). Control of particulate size is critical to maintain 
rheology and prevent undesirable texture attributes, such as coarseness and grittiness, because of this (Jeffery, 1993; Servais et al., 2002; Afoakwa et al., 2007).

Many commonplace ingredients will greatly contribute to the rheology of fat-based confections. Emulsifiers aid in processability and minimization of raw material costs by improving the flow properties of chocolate and vegetable fat-based suspensions (mostly used as coatings) through lowering the friction between the particulates suspended in the liquid fat phase. As a result, $\tau_{0}$ is reduced and ease of flow within these suspensions is permitted, even at low total fat content.

Using a sugar particle as an example, to coat dispersed particulates, the hydrophobic moiety of the emulsifier will protrude into the fat phase whereas its hydrophilic moiety will adsorb onto the surface of the sugar particle. In general terms, surface activity from an emulsifier will depend on its chemical structure, namely the balance in size between the hydrophilic headgroup and hydrophobic tailgroup. Those with larger headgroups will bind more strongly to sugar whereas others will be less strongly attached and may even be removed by the addition of different emulsifiers. Similarly, tailgroup length may affect the flow properties in different ways, implying that emulsifiers which are especially beneficial towards $\tau_{0}$ may be poor towards $\eta$ and vice versa (Beckett, 2008).

Lecithins are derivatives of sn-glycero-3-phosphates with one or two fatty acids (FAs) attached to a glycerol backbone and an alcohol esterified to a phosphate group at the third glycerol position. They are commonly used to stabilize particulates in oil-continuous dispersions (e.g., sugar particles in CB), as they align into layers at the solid surface to reduce 
Table 1.1 Effect of soy lecithin concentration on $\tau_{0}$ and $\eta$ of milk chocolate (Schantz and Rohm, 2005).

\begin{tabular}{ccc}
\hline Concentration $\left(\mathbf{g} \cdot \mathbf{k g}^{-\mathbf{1}}\right)$ & $\boldsymbol{\tau}_{\mathbf{0}}(\mathbf{P a})$ & $\boldsymbol{\eta}(\mathbf{P a} \cdot \mathbf{s})$ \\
\hline 0 & 79.60 & 12.30 \\
1 & 19.50 & 4.18 \\
2 & 11.80 & 3.63 \\
3 & 10.20 & 3.28 \\
4 & 9.00 & 3.04 \\
5 & 9.15 & 2.89 \\
6 & 9.59 & 2.79 \\
7 & 10.30 & 2.71 \\
\hline
\end{tabular}

$\eta$ stemming from particle aggregation (Johansson and Bergenståhl, 1992). For example, in chocolate manufacture, cocoa liquor (or mass) is mixed with sugar, additional $\mathrm{CB}$, milk powder, and soy lecithin. Through refining and conching, the action of shear and coating of sugar with fat and soy lecithin improves chocolate flow properties. The addition of soy lecithin decreases $\tau_{0}$ and $\eta$ in chocolate until a maximum concentration of $4 \mathrm{~g} \cdot \mathrm{kg}^{-1}$. Beyond this point, continued addition begins to increase $\tau_{0}$ (Table 1.1) (Chevalley, 1999; Schantz and Rohm, 2005). This is attributed to the micelles produced from lecithin phospholipids that disrupt particulate flow (Vernier, 1998).

PGPR is a synthetic food-grade emulsifier consisting of polyglycerol as the hydrophilic group and interesterified ricinoleic FAs as the hydrophobic group (molar mass between 1200-2000 $\mathrm{g} \cdot \mathrm{mol}^{-1}$ ) (Middendorf et al., 2015). It is increasingly being sought as a replacement given its $\eta$-reducing effects as with soy lecithin but at lower $\%(w / w)$ values. For example, Ghorbel et al. (2011) found PGPR decreased $\tau_{0}$ more significantly than either soy lecithin or total fat content in molten milk chocolate. Both emulsifiers had similarly influenced $\eta$. The interaction between emulsifier and fat on the one hand - and those between the emulsifier and solid particles on the other-play an important role not only in 
determining chocolate flow properties but also texture of the final product. Rousset et al. (2002) stated that PGPR enhanced the lipophilicity of sugar particles thereby decreasing sugar-sugar interactions and increasing the fluidity of the fat-based suspension. Finally, Middendorf et al. (2015) concluded that PGPR interacts with CB immobilized on the surface of sugar to form pillow-like deposits, which act as spacers to reduce interaction between sugar particles. In addition to providing creaminess, milk solids also impact rheology where casein, which possesses both polar and non-polar sites, behaves similar to an emulsifier (Haylock and Dodds, 1999).

An increase in demand of low-calorie confections has resulted in many sugar-free products (Zumbé and Brinkworth, 1993; Sokmen and Gunes, 2006). Sugar substitutes, which include bulk sweeteners such as sugar alcohols, e.g., sorbitol and xylitol, and saccharide alternatives, e.g., trehalose and isomaltulose (Olinger, 1994; Kroger et al., 2006; Aidoo et al., 2013), have a negative impact on rheology (Clayton and Conn, 2005). For example, sugar alcohols were found to reduce the quality of chocolate and result in poorer rheology (Zumbé and Brinkworth, 1993). A further study showed that maltitol produced a $\eta$ most similar to sucrose whereas isomalt significantly increased it (Sokmen and Gunes, 2006). In another study, the substitution of sucrose with steviol glycosides, which are natural sweet-tasting compounds isolated from the plant Stevia rebaudiana, in combination with inulin and dextrose as bulking agents was not found to significantly alter hardness of the chocolate (Shah et al., 2010).

Moisture often has a dire effect on fat-based confections. It causes dispersed particulates to cluster through attractive polar forces and capillary action, resulting in poor mixing and increased particle heterogeneity as well as deleterious effects on $\eta$ (Nováková et al., 2002). 
This is in large part due to the low affinity sugar has for the continuous phase. The effect of moisture has also restricted the usage of hygroscopic low-calorie ingredients such as glucose or fructose (Afoakwa et al., 2007). To counteract this effect, confections are formulated with a higher fat content, though this is clearly not cost-effective. For example, CB is adjusted by a three-fold addition for every one-fold addition of unwanted moisture to correct its rheology (Beckett, 2000; Aidoo et al., 2012). Approaches such as high intensity conching (Nováková et al., 2002) and ultrasound (Yucel and Coupland, 2011) may also remove these aggregates.

A nascent area is that of the role of micro-viscosity $\left(\eta_{\mu}\right)$ and rheology on particle networks. Characterizing the micro-rheological properties of liquid-state oil may improve our understanding of the role of confinement and non-fat ingredient interactions on fat crystallization. Therefore, it is important to isolate this behaviour from the bulk rheology as much as possible. It was previously reported that oil $\eta_{\mu}$ was influenced by the fractal dimension of fat-oil mixtures and not their SFC. Relying on the twisted intramolecular charge transfer properties of the molecular rotor citrus red, $\eta_{\mu}$ was determined to increase as oil confinement and fractal dimension increased (Du et al., 2014). While determination of oil $\eta_{\mu}$ may possibly give insight into its migration and impact on crystallization in systems containing non-fat ingredients, any existing study remains to be published.

Large deformation experiments, e.g., cone penetrometry and two-plate compression, are used to determine the textural properties of foods, e.g., mechanical firmness and adhesiveness, and are often used to corroborate sensory tests (Hayakawa and DeMan, 1982; Rousseau and Marangoni, 1999; Liang and Hartel, 2004; Omar et al., 2005). These experiments may also be used in conjunction with rheology (Beckett, 2000; Bourne, 2002). 
The main limitation with texture analysis is its inability to reproduce chewing and explore salivary effects, which can invert phases within fat-based confections, changing the way in which they are perceived (Lee and Pangborn, 1986; Carvalho-da-Silva et al., 2013). Tribology, which studies the interaction between food and oral epithelia, has been developed to minimize this challenge. The tribological properties of some fat-based confections have been studied, though much work remains to be done in this area (Carvalho-da-Silva et al., 2011; Lee et al., 2002).

\subsection{Fats and Oils}

\subsubsection{Molecular Structure}

Like all food fats, confectionery fats are mixtures of higher- and lower-melting triacylglycerols (TAGs) where FAs are esterified to a glycerol skeleton (Figure 1.2) (Timms, 1984). These molecules conform to an asymmetric tuning fork or chair geometry due to steric hindrance at the sn-2 position (Larsson et al., 2006). Hindrance is exacerbated with unsaturation at this position, e.g., oleic acid in CB TAGs, which is partially responsible for its rapid melting behaviour in the mouth (Smith and Dahlman, 2005; Afoakwa et al., 2007).

Mixtures of fats may be simple, consisting of just a few key TAGs, e.g., CB (Sato et al.,1989; Loisel et al., 1998; Beckett, 2000; Smith and Dahlman, 2005), or complex with several hundreds, e.g., milkfat (MF) (Gresti et al., 1993). As composition becomes more complex, the thermal profile of the fat becomes less defined and broader, e.g., MF melts between - 40 and $40^{\circ} \mathrm{C}$ (Larsson, 1994). Both origin and terroir, e.g., temperature and rainfall, can alter the composition of the source, influencing the physical properties of the fat (Table 1.2) (Timms, 2003; Marty and Marangoni, 2009). 


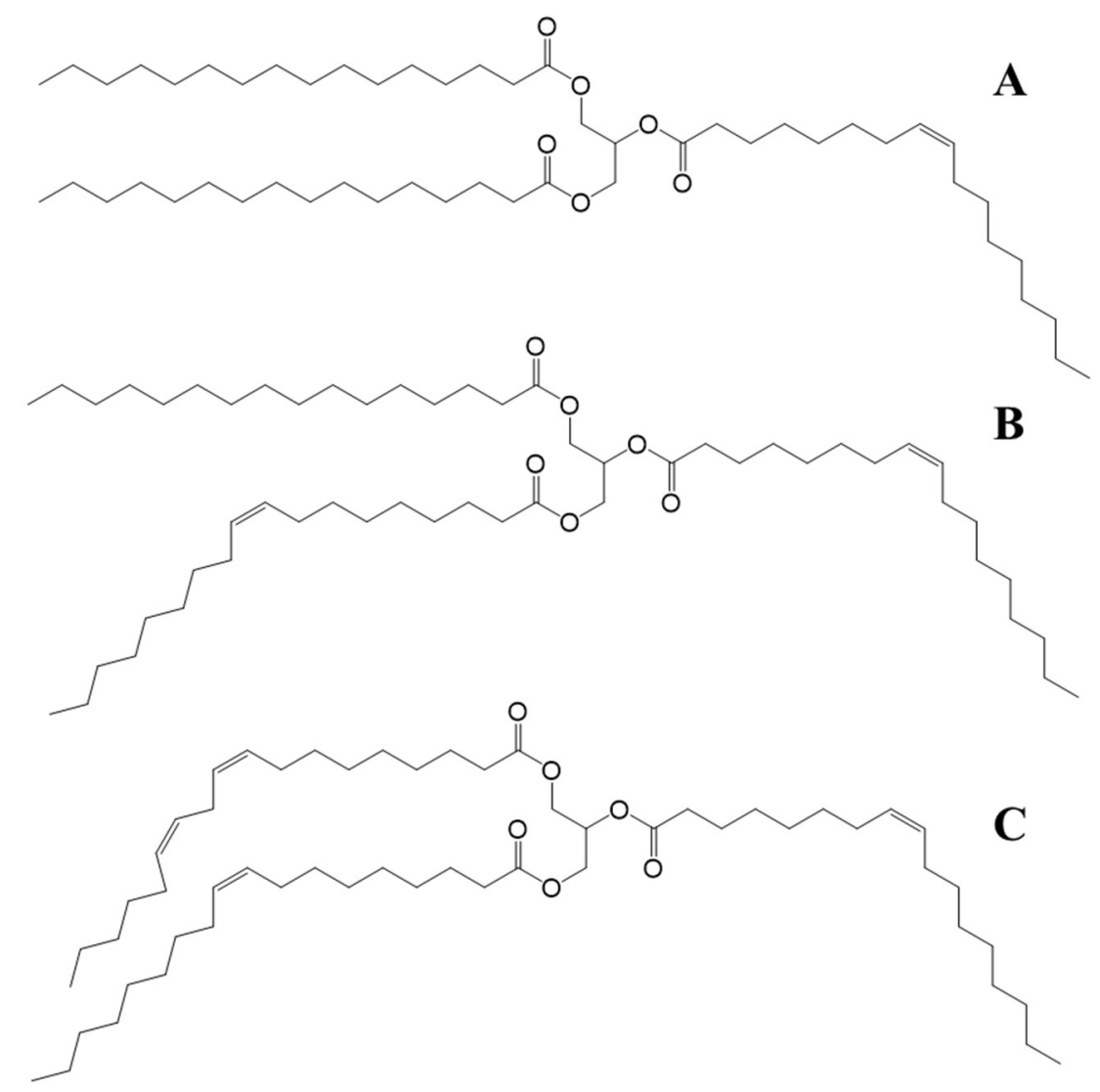

Figure 1.2 Molecular structure of (A) 1,3-dipalmitoyl-2-oleoylglycerol, (B) 1-palmitoyl2,3-dioleoylglycerol, and (C) 1-palmitoyl-2-oleoyl-3-linoleoylglycerol.

Common minor components include partial acylglycerols, e.g., monoacylglycerols (MAGs) and diacylglycerols (DAGs), free fatty acids (FFAs), and other components, e.g., carotene, tocopherol, etc. (Gee, 2007; Gibon et al., 2007; Kellens et al., 2007; Smith et al., 2011). DAGs have higher slip points than TAGs, which can negatively impact the intended application (Ab Latip et al., 2013). For example, in one study the slip points of commercial and DAG-enriched margarines were determined to be $30.5 \pm 0.2$ and $35.5 \pm 0.1{ }^{\circ} \mathrm{C}$, respectively (Saberi et al., 2012). With the consideration of oral temperature, a DAGenriched margarine would have a longer, waxier thermal profile. DAGs are also believed to slow fat crystallization, although this mechanism is not fully understood (Siew and $\mathrm{Ng}$, 
Table 1.2 Effect of CB origin on TAG composition (Marty and Marangoni, 2009). ${ }^{1.2}$

\begin{tabular}{ccccccc}
\hline TAG (\%) & Brazil & China & Ecuador & Ivory Coast & Malaysia & Nigeria \\
\hline OOL & 0.5 & 1.0 & 1.1 & 0.6 & 1.0 & 1.8 \\
PLP & 1.5 & 1.1 & 1.3 & 1.5 & 1.2 & 1.1 \\
OOO & 5.1 & 0.8 & 1.9 & 1.8 & 1.4 & 1.2 \\
POP & 22.8 & 23.5 & 24.1 & 24.3 & 22.7 & 23.1 \\
PPP & 0.0 & 0.1 & 0.0 & 0.0 & 0.0 & 0.1 \\
SOO & 7.0 & 1.7 & 2.1 & 2.1 & 1.8 & 1.2 \\
POS & 34.1 & 37.0 & 37.3 & 37.3 & 36.9 & 37.3 \\
PPS & 0.1 & 0.2 & 0.1 & 0.1 & 0.1 & 0.2 \\
SOS & 28.3 & 33.1 & 31.1 & 31.4 & 33.4 & 32.5 \\
SSP & 0.1 & 0.4 & 0.3 & 0.3 & 0.4 & 0.5 \\
SSS & 0.6 & 1.0 & 0.8 & 0.7 & 1.1 & 1.1 \\
\hline
\end{tabular}

1999; Tshiamala, 2013). Though emulsifiers are a mainstay of the fat and oil industry, there remains a dearth of information on their structure-function relationship and the mechanisms by which they influence TAG nucleation and growth. In this regard, they may significantly impact fat nucleation, crystal growth, or both, depending on their composition, added amount, and mechanism of action (Smith et al., 2011).

\subsubsection{Polymorphism and Morphology}

The key polymorphs identified in most fats and oils, in order of increasing stability, are the $\alpha$-hexagonal, $\beta^{\prime}$ - orthorhombic, and $\beta$-triclinic forms (Figure 1.3) (Talbot, 1999; Bresson et al., 2011; Tshiamala, 2013). These different configurations describe the lateral packing of molecules and provide the fingerprint short spacings. While polymorph subtypes have been reported in pure TAG systems, e.g., sub- $\alpha$, pseudo- $\beta^{\prime}$, sub- $\beta$, etc., they are not universally accepted (D’Souza et al., 1990).

\footnotetext{
1.2 Oleic $(\mathrm{O})$, linoleic $(\mathrm{L})$, palmitic $(\mathrm{P})$, and stearic $(\mathrm{S})$.
} 


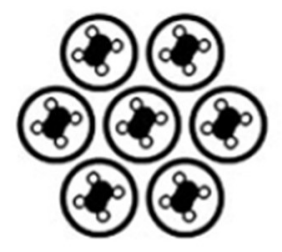

Hexagonal $(\mathrm{H})$ a-form

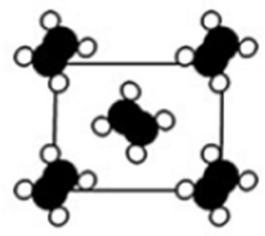

Orthorhombic $\left(\mathrm{O}^{\perp}\right)$

$\beta^{\prime}$-form

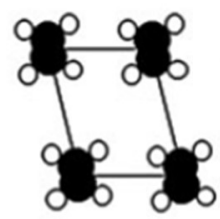

Triclinic (T/I)

$\beta$-form

Figure 1.3 Polymorphic forms of TAGs (Bresson et al., 2011).

X-ray diffraction (XRD) is used to identify fat crystal polymorphs (Figure 1.4) (D'Souza et al., 1990; Schenk and Peschar, 2004; Da Silva et al., 2009). With wide-angle XRD, the simplest definition is based on short spacings where one strong peak at $4.15 \AA$ is observed for $\alpha$ crystals, peaks at 4.2 and $3.8 \AA$ for $\beta^{\prime}$ crystals, and one strong peak at $4.6 \AA$ and two lesser peaks at 3.9 and $3.7 \AA$ for $\beta$ crystals (Larsson, 1966; Hernqvist, 1988a; DeMan, 1992; Verstringe et al., 2012). These forms are driven by van der Waals forces to further pack into bilayer or trilayer lamellae either two to three alkyl chains thick, respectively, which is interpreted as the long spacings of the crystal (Talbot, 1999; Larsson et al., 2006).

Other than XRD, many techniques can be used to characterize the crystallization behaviour of fats (Van Malssen et al., 1994, 1999; Loisel et al., 1998; Van Langevelde et al., 2001a, 2001b), including differential scanning calorimetry (DSC) (Foubert et al., 2008; De Cock, 2011; Tshiamala, 2013), Raman spectroscopy (Kobayashi, 1988; Bresson et al., 2005, 2006, 2011; $\mathrm{Hu}$ et al., 2005; Schoukens and De Clerck, 2005; Da Silva et al., 2009), and dilatometry (Wille and Lutton, 1966), although this final technique is rarely used nowadays.

Polymorphs are associated with specific fat crystal morphologies under static crystallization. For example, $\alpha$ crystals are least dense and form platelets $\sim 5 \mu \mathrm{m}$ in length (Rousseau et al., 

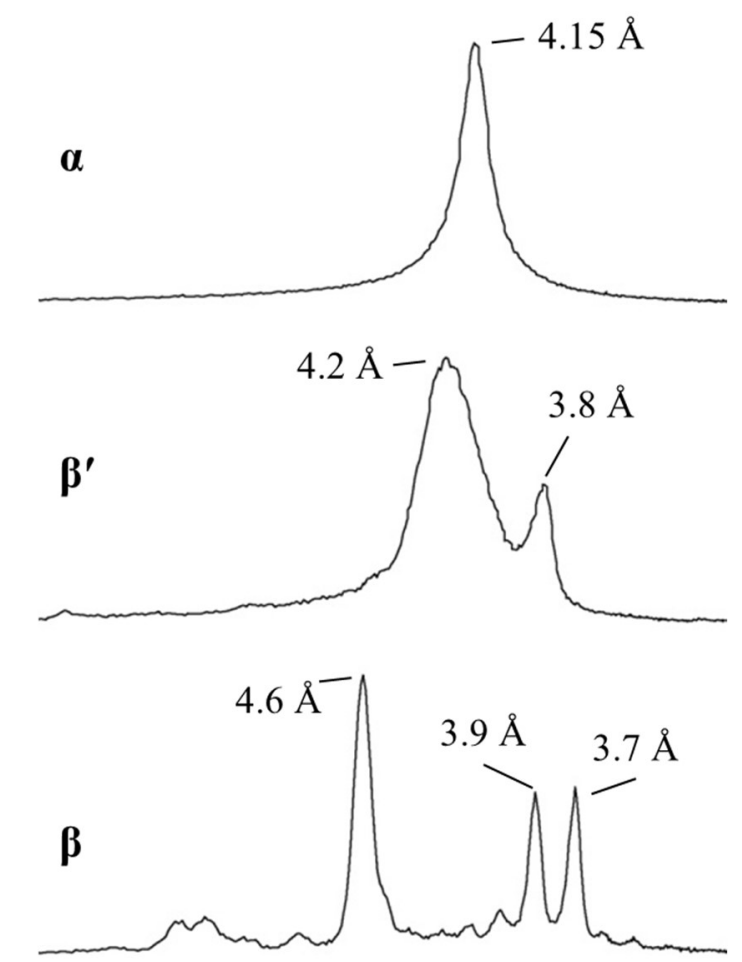

Figure 1.4 Diffractograms of different tristearin polymorphs (adapted from Da Silva et al., 2009).

2005). Both $\beta^{\prime}$ and $\beta$ crystals are acicular where growth is more energetically favoured along its length. These needle-like crystals are typically 1-2 and potentially upwards of $50 \mu \mathrm{m}$ in length for $\beta^{\prime}$ and $\beta$, respectively. Morphology may affect textural properties of bulk oils. Rounded crystals, such as those shaped by shear, will flow past each other more easily to produce a smoother texture whereas sharper, more angular crystals will fracture and become brittle. For example, the ideal textural properties for palm-based margarines, including proper smoothness and spreadability, are obtained if $\beta^{\prime}$ crystals are formed. In more complex foods, the effect of crystal morphology on texture is not fully understood because of factors such as multiple phases, ingredient interactions, and compartmentalization (i.e., the internal segregation within a colloidal by its dispersed phase) (Hartel, 2001). 
Table 1.3 Melting point and chain-length packing of CB polymorphs (Afoakwa et al., 2007).

\begin{tabular}{ccc}
\hline Polymorph & Melting point $\left({ }^{\circ} \mathbf{C}\right)$ & Chain-length \\
\hline$\gamma$ & $16-18$ & 2 \\
$\alpha$ & $21-22$ & 2 \\
$\beta^{\prime}{ }_{2}$ & 25.5 & 2 \\
$\beta^{\prime}{ }_{1}$ & $27-29$ & 2 \\
$\beta_{2}$ & $34-35$ & 3 \\
$\beta_{1}$ & 36 & 3 \\
\hline
\end{tabular}

As in other fats, polymorphism in confectionery fats is monotropic evolving from less to more stable polymorphs. CB polymorphism has been studied extensively because of its close association to organoleptic properties and shelf life. A previous XRD study (Wille and Lutton, 1966), later to be confirmed by DSC (Huyghebaert and Hendrickx, 1971), showed a total of six polymorphs to exist in CB, i.e., $\gamma, \alpha, \beta^{\prime}{ }_{2}, \beta^{\prime}{ }_{1}, \beta_{2}$, and $\beta_{1}$, melting over a range of 16 to $36^{\circ} \mathrm{C}$ (Table 1.3), with $\beta_{2}$ crystals exhibiting optimal macroscopic properties (Smith and Dahlman, 2005). The triple chain-length packing of higher order polymorphs further allows for higher thermodynamic stability and lower occupied volume compared to double chain-length packing (Talbot, 1999). With proper tempering, $\beta_{2}$ crystals initially form at 1 $3 \%(\mathrm{v} / \mathrm{v})$, establishing a template for further crystallization of the remaining melt (Svanberg et al., 2013). More contemporary methods use pre-made $\beta_{2}$ crystals to seed the melt and facilitate proper crystallization of the chocolate mass (Windhab, 1999; Zeng, 2000). Svanberg et al. (2011a) showed that seeded samples of molten chocolate formed multiple nucleation sites and increased in morphological homogeneity. In the absence of seeding, large spherical crystals were observed in some areas, but were absent in others.

Importantly, $\beta_{2}$ crystals contribute to a glossy finish, clean fracture, i.e., snap, quick melting at oral temperature, flavour release, and contraction for demoulding of shelled confections 
(Timms, 1984, 2003; Seguine, 1991; Stapley et al., 1999; Talbot, 1999; Beckett, 2000, 2008;

Khan and Rousseau, 2006; James and Smith, 2009). Shelf life is also prolonged because of the ability for $\beta_{2}$ crystals to restrict liquid oil from migrating to the surface and promote fat bloom, characterized by the onset of large $\beta_{1}$ crystals, i.e., $>5 \mu \mathrm{m}$, that have the ability to diffuse light, giving a dull, greyish appearance on the surface (Cebula and Ziegleder, 1993; Lohman and Hartel, 1994; Loisel et al., 1997; Ziegleder, 1997; Hartel, 1999; Sato, 2001; Timms, 2003; Dibildox-Alvarado et al., 2004; Lonchampt and Hartel, 2004; Smith et al., 2007; Nopens et al., 2008; Dahlenborg et al., 2011; Glicerina et al., 2013). Furthermore, with $\beta_{1}$ crystals, melting point is increased and the product adopts a grittier texture (Afoakwa et al., 2009). Internally, $\beta_{1}$ crystals may also form but are typically smaller and more irregularly shaped (Jewell, 1972; Hartel, 1999; Lonchampt and Hartel, 2004; James and Smith, 2009; Dahlenborg et al., 2011; Dahlenborg, 2014). With proper storage, it may take years for properly-tempered chocolate to produce enough bloom-inducing $\beta_{1}$ crystals (Bomba, 1993; Subramaniam, 2000; Rousseau et al., 2010). With under-tempering, this process occurs in just days (Vaeck, 1960; Campbell, 1967; Hernqvist, 1988b; Rousseau, 2007; Bresson et al., 2011), as $\beta^{\prime}{ }_{1}$ crystals rapidly form due to the lack of a proper template and undergo a $\beta^{\prime}{ }_{1} \rightarrow \beta_{2}$ transition on the surface of chocolate (Schlichter-Aronhime and Garti, 1988; Seguine, 1991; Hodge and Rousseau, 2002).

\subsubsection{Crystallization}

Fat crystallization may occur from either melts or solutions (Figure 1.5) (Himawan et al., 2006; Kellens et al., 2007). This process is driven by the reduction of activation free energy as the system is shifted from equilibrium and either the melt is cooled below its melting 


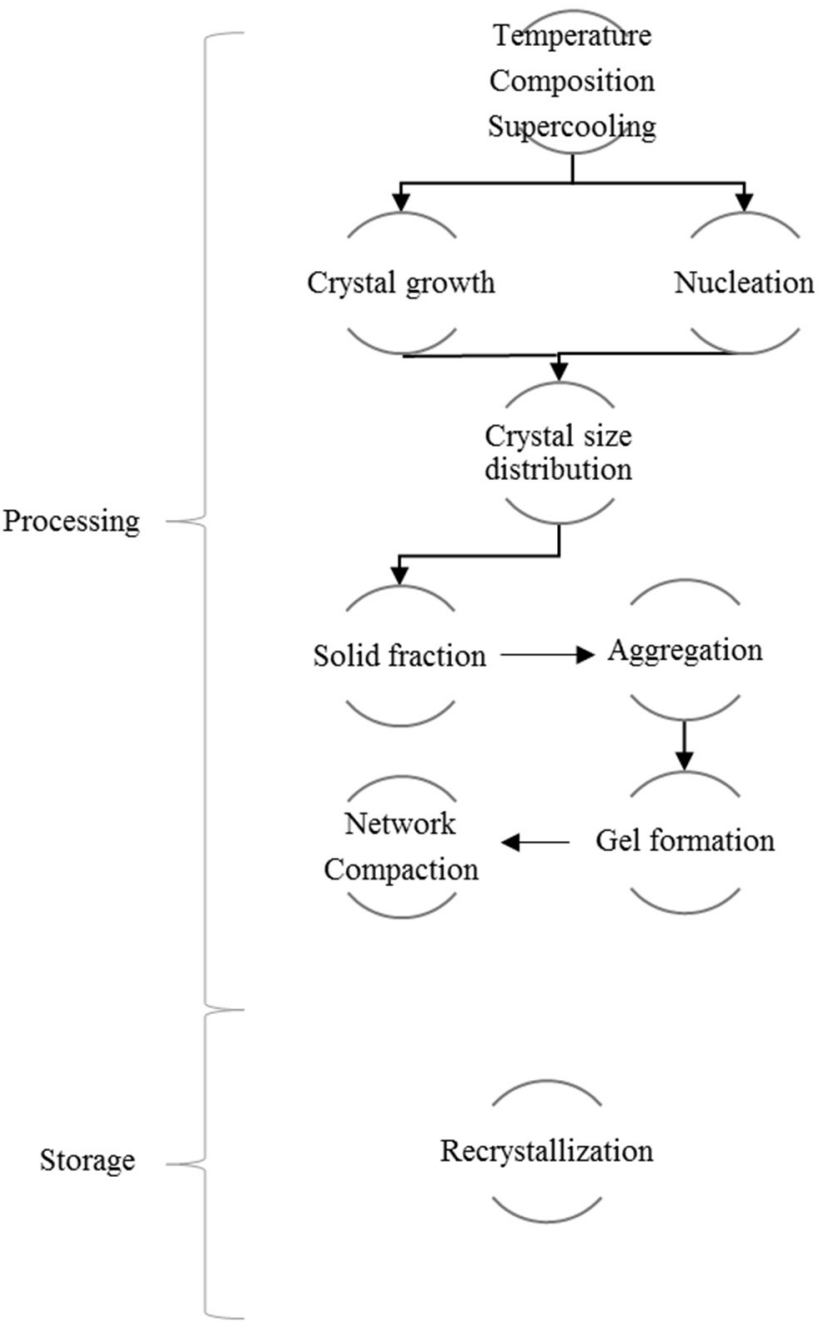

Figure 1.5 Timeline of events during fat crystallization and storage (adapted from Himawan et al., 2006).

point or the solution has reached saturation. For melts, the chemical affinity $(\Phi)$, which is the driving force promoting TAGs to associate, is expressed by:

$$
\Phi=\Delta H_{f} \frac{\left(T_{f}-T\right)}{T_{f}}
$$

where $\Delta H_{f}$ is the latent heat of fusion, $T_{f}$ is the melting point, and $T$ is the temperature of the system (Hartel, 2001). For solutions, this is expressed by: 


$$
\Phi=R T \ln \left(\frac{\gamma X}{\gamma_{s} X_{S}}\right) \approx R T \ln \left(\frac{C}{C_{S}}\right)
$$

where $R$ is the gas constant, $\gamma$ is the activity coefficient, $\gamma_{s}$ is the activity coefficient at equilibrium, $X$ is the mole fraction, $X_{S}$ is the mole fraction at equilibrium, $C$ is the concentration, and $C_{S}$ is the saturation concentration (Hartel, 2001). Obviously, as $T$ is reduced in either melts or solutions, so is $\Phi$. In systems of great TAG diversity, crystallization proceeds from both melt and solution. This is explained by the presence of lower-melting TAGs serving as solvent for fat crystals composed of higher melting TAGs. Butter is just one example of a mixed system where crystallizations from both melt and solution simultaneously occur (Hartel, 2001).

The first step in crystallization is primary nucleation where molecules either combine from the molten state, i.e., homogeneous, or form on the surfaces of impurities, i.e., heterogeneous (Metin and Hartel, 2005; Basso et al., 2010). Liquid-state TAGs assemble through local energy fluctuations into embryos under homogeneous nucleation (Walton, 1969; Zettlemoyer, 1969; Mullin, 1993). This process is reversible, but the rate of association eventually exceeds that of dissociation as the system continues to shift away from equilibrium. These embryos must form prior to the formation of stable nuclei of a critical size. For melts, this critical size $\left(r_{c}\right)$ is estimated by:

$$
r_{c}=\frac{2 \sigma_{s} v}{\Phi}
$$

where $\sigma_{s}$ is the interfacial tension and $v$ is the molecular volume (Hartel, 2001). The rate of homogeneous nucleation $\left(J_{h o m}\right)$ from the molten state is: 


$$
J_{\text {hom }}=A \exp \left\{-\frac{\Delta G_{c}}{k T}+\frac{\Delta G_{v}^{\prime}}{k T}\right\}
$$

where $k$ is Boltzmann's constant, $\Delta G_{c}$ is the critical free energy for a stable nucleus, and $\Delta G^{\prime}{ }_{v}$ is the free energy for molecular diffusion. Nucleation is further influenced by polymorphism where the rate increases with lower order (Hartel, 2001). A reduced $\sigma_{s}$ in $\alpha$ crystals is one suggestion behind their tendency to nucleate before either $\beta^{\prime}$ or $\beta$ crystals (Sato, 1988), where their hexagonal mobility may significantly reduce interfacial energy.

The theory behind the rate of heterogeneous nucleation $\left(J_{h e t}\right)$ is a modification of homogeneous nucleation, such that:

$$
J_{\text {het }}=A \exp \left\{-\frac{\Delta G_{c}^{\prime}}{k T}\right\}
$$

where $\Delta G^{\prime}{ }_{c}$ is the critical free energy for heterogeneous nucleation (Hartel, 2001). $\Delta G^{\prime}{ }_{c}$ in this case is the combination of tensions at the melt-foreign surface, crystal-foreign surface, and melt-crystal interfaces (Garside, 1987). $\Delta G^{\prime}$ may be explained by:

$$
\Delta G_{c}^{\prime}=\Delta G_{c}\left\{\frac{1}{4}(2+\cos \theta)\left(1-\cos ^{2} \theta\right)\right\}
$$

where $\theta$ represents the angle of wetting between crystal and nucleating solid. This angle may be calculated by:

$$
\cos \theta=\frac{\sigma_{s l}-\sigma_{c s}}{\sigma_{c l}}
$$

where interfacial tensions for melt-foreign surface, crystal-foreign surface, and melt-crystal are $\sigma_{s l}, \sigma_{c s}$, and $\sigma_{c l}$, respectively. The presence of a foreign surface, such as a sugar particle, should lower $\theta$, which in turn requires fewer liquid-state TAGs to assemble a nucleus of critical size. In return, $\Delta G_{c}^{\prime}$ is lowered and nucleation occurs more easily (Hartel, 2001). 
Furthermore, a greater degree of lattice matching, i.e., physical or chemical complementarity, between the foreign surface and fat crystal may impact nucleation of the system (Metin and Hartel, 2005).

Foreign surfaces that facilitate nucleation may be inherent to the system, e.g., dust, dirt, etc., or they may be intentionally added. For example, heterogeneous nucleation of fat crystals off the surface of sugar particles was observed in non-seeded CB-sugar systems (Svanberg et al., 2011a). It has been theorized that one catalytic impurity per cubic millimetre results in a nucleation large enough to ensure rapid phase transition at a given supersaturation (Walstra, 2003). Homogeneous nucleation rarely proceeds in food systems because of this (Land et al., 1999; Cacciuto et al., 2004; Eremina et al., 2005; Rak et al., 2005).

The deviation from equilibrium required for nucleation is reduced in the presence of foreign surfaces because they provide active sites that lower the energy barrier by facilitating proper orientation of liquid-state TAGs (Bowser, 2006; Rousseau and Sonwai, 2008; Svanberg et al., 2011a, 2011b; Fernandes et al., 2013; Hartel, 2013). For example, crystallization induction time is reduced in the presence of sugar for palm oil (PO) (Figure 1.6), cocoa solids for CB (Svanberg et al., 2011a), common minor components of fats and oils (Savage and Dimick, 1995), and solvents such as acetone (Hartel, 2001). Furthermore, local shear rates near non-fat ingredient surfaces increase nearly two-fold, providing enough energy to promote transition to higher ordered polymorphs (Mazzanti et al., 2003; Dhonsi and Stapley, 2006). The effect of various lipophilic molecules on nucleation has been previously reviewed (Smith et al., 2011; Yoshikawa et al., 2014). 


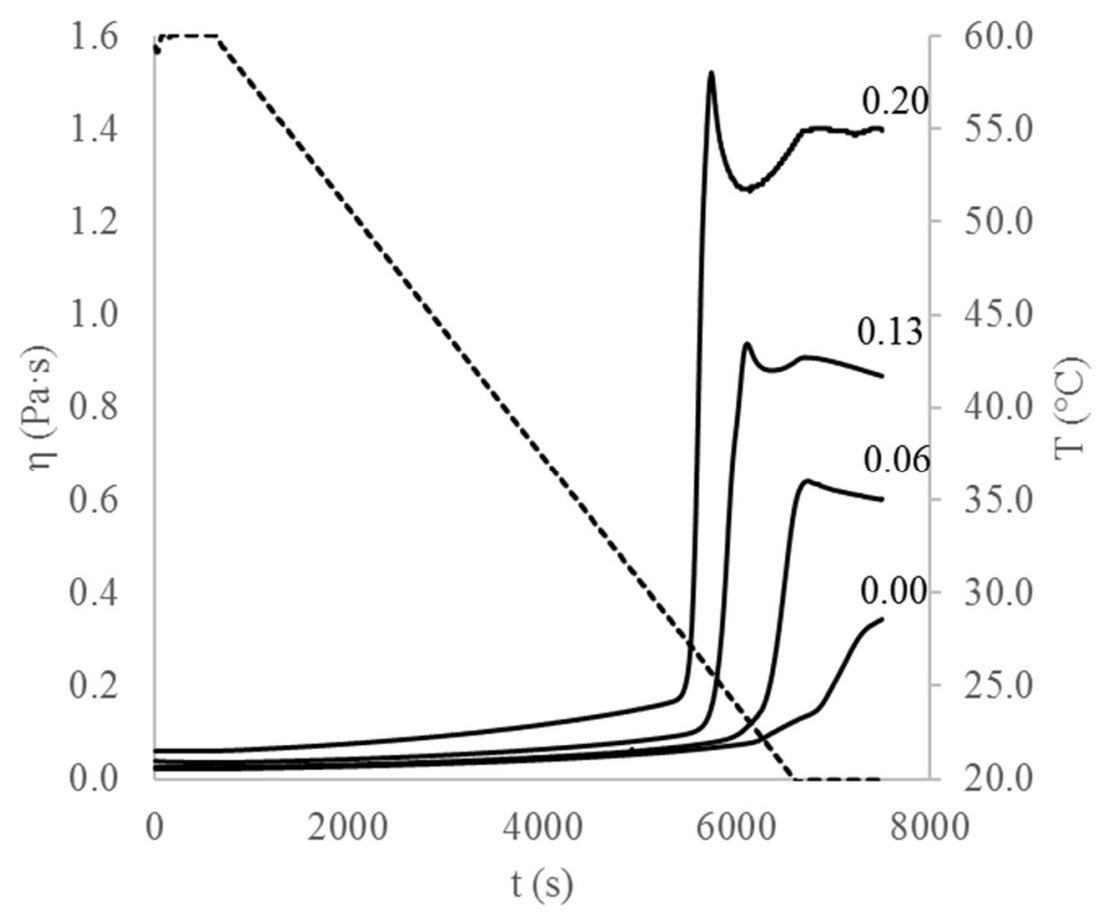

Figure 1.6 Effect of sugar volume fraction on PO crystallization during cooling (unpublished data).

The effect of inorganic additives on trilaurin (LLL) crystallization was recently studied by Yoshikawa et al. (2014). These additives appeared to enhance crystallization and the $\beta^{\prime} \rightarrow \beta$ transition. Furthermore, there existed an interaction between type of additive and TAG orientation on the surface of LLL crystals. XRD studies revealed greater peak intensities for long spacing over short spacing in the presence of talc, suggesting orientation where lamellar planes were parallel to additive surface. In contrast, these planes grew normal to the surface of graphite (Figure 1.7) (Yoshikawa et al., 2014). Crystallization suppression may sometimes be observed in the presence of an additive (Cacciuto et al., 2004). For example, soy lecithin has suppressed fat crystallization of CB-sugar models (Dhonsi and Stapley, 2006). 

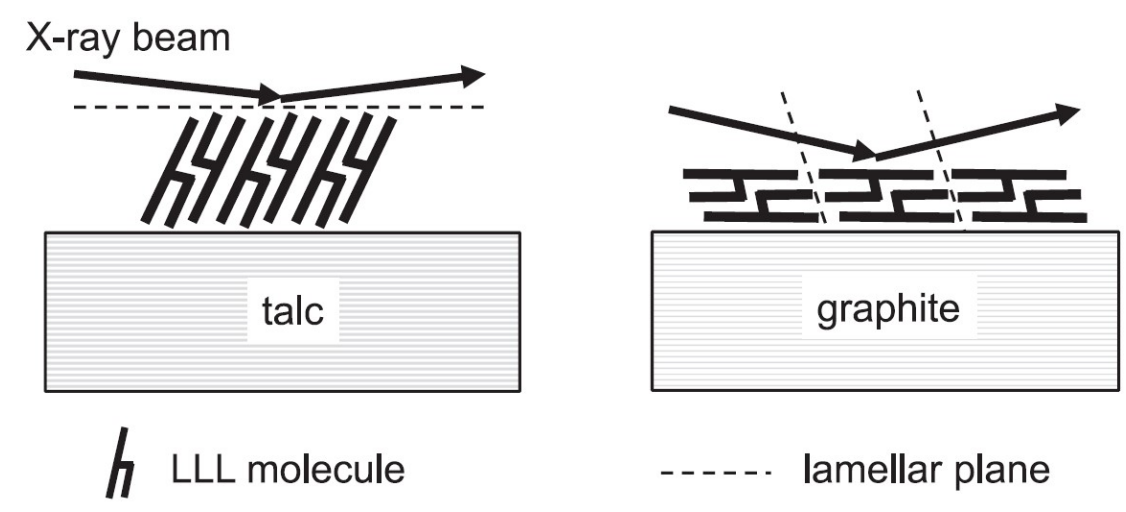

Figure 1.7 Effect of additive on lamellar orientation of LLL (Yoshikawa et al., 2014).

Crystals are fewer but larger in size if nucleation is slow, hindering their ability to flow across each other with minimal friction and causing products to become grainy or coarse in texture. For example, the textural quality of margarines is significantly reduced if crystals exceed $10 \mu \mathrm{m}$ in length (Walstra, 2003). In contrast, high nucleation rates result in many smaller crystals and a pasty consistency (Hartel, 2001). The rate of nucleation may be quantified by refractive index, turbidimetry, or light scattering although this has been met with challenges (James, 1982).

Secondary nucleation depends on the presence of existing fat crystals and can occur in parallel to primary nucleation (Larson, 1981; Metin and Hartel, 2005; Basso et al., 2010). While questions remain as to how exactly this occurs (Randolph and Larson, 1988), some mechanisms suggest secondary nucleation proceeds by micro-attrition where primary crystals fragment under high shear, feeding the secondary nuclei (Walstra, 2003). Following primary and secondary nucleation and crystal formation is crystal growth where liquid-state TAGs continue to incorporate onto crystal surfaces and heat of fusion is absorbed until an equilibrium is reached (Tavare, 1995; Hartel, 2001, 2013). The energy that is released from heat of fusion may also facilitate the transition of lower order polymorphs into higher states. 
Eventually these crystals agglomerate to form a network in which the remaining liquid phase is entrapped (Metin and Hartel, 2005; Kellens et al., 2007).

Following the formation of stable nuclei, growth and association of crystallized lamellae result in the formation of crystal nanoplatelets (Acevedo and Marangoni, 2010; Acevedo et al., 2011; Marangoni and Wesdorp, 2013). These nanoplatelets organize into stack-like structures by means of molecular van der Waals forces which then go on to assemble into crystallites that cluster via colloidal van der Waals forces to form fat crystals. Aggregation and sintering (i.e., formation of solid bridges) between crystals results in the formation of a three-dimensional fat crystal network (Pink et al., 2013; Tarabukina et al., 2009).

Fat crystal networks are easily characterized using polarized light microscopy in bulk systems (Chen et al., 2002; Tang and Marangoni, 2007). Microscopy is used to better understand food structure and interactions at the molecular level and how they impact macroscopic properties (Flint, 1994; Stanley et al., 1998; Aguilera and Stanley, 1999; Morris, 2007). For fat- and oil-based products, factors such as fat crystal morphology and size distribution of non-fat ingredients are important considerations for product developers (Rousseau, 2007). While this distribution may sometimes be difficult to obtain, sizing by either optical microscopy, gravimetry via sieve stacks, or laser diffraction is possible for objects larger than a few microns.

\subsubsection{Compositional and Processing Effects}

The crystallization pathway is heavily influenced by both composition and processing, e.g., cooling rate, shear, etc. (Hernqvist, 1984; Awua, 2002; Campos et al., 2002; Dhonsi and Stapley, 2006; Kellens et al., 2007; Pérez-Martínez et al., 2007). For example, TAG 
composition can impact polymorphism where larger diversity favours metastable $\beta^{\prime}$ crystal formation (Rousseau, 2007). Furthermore, while shear can complicate polymorphic assignment based on morphology, the rate of crystallization is increased through its ability to facilitate mass transfer and higher ordered polymorphs proliferate (Sato, 2001; Mazzanti et al., 2003; De Graef et al., 2006; Tarabukina et al., 2009). Mass transfer of TAGs during crystal growth is hypothesized to occur either through diffusion, convection, or a combination of the two (Mullin, 1993). The rate of crystal growth $\left(R_{c}\right)$ has been explained by the simple model:

$$
R_{c}=\frac{K(\sigma-1)}{\eta}
$$

where $K$ is a constant and $\sigma$ is the degree of supersaturation (Mathlouthi and Genotelle, 1995). Assuming $\sigma$ is constant, $R_{c}$ is reduced as $\eta$ of the system increases.

Large kinetic factors, e.g., large cooling rate, favour the formation of many small crystals composed of lower ordered polymorphs according to Ostwald's Step Rule (Bennett et al., 1965; Sato, 2001). DSC has been used to verify this where a small exotherm corresponding to the $\alpha$ polymorph was observed under a higher degree of supercooling (Hartel, 2001). Liquid-state TAGs quickly incorporate within growing crystals under these conditions, resulting in lamellar occlusions and disorganization (Bennett et al., 1965). Transition of these crystals to a higher ordered polymorph may be on the order of months because of steric hindrance and larger energy barrier (Sato, 1993; Hartel, 2001).

Larger but fewer crystals of higher polymorphic order form under smaller cooling rates (Litwinenko et al., 2002; Metin and Hartel, 2005). Furthermore, these conditions minimize thermal gradients (Kleinert, 1961) and allow the complete formation of a lamella before 
another layer begins (Marangoni, 2005). While this may seem favourable, one potential caveat to a small cooling rate is the coexistence of different polymorphs, which may weaken the overall crystal network (Fessas et al., 2005). The effect of cooling rate on fat crystallization has been studied further (Baldino et al., 2010).

Cooling endpoint temperature is also an important consideration in processing. For example, $\alpha$ crystals form readily in some oils, e.g., $\mathrm{PO}$, at an endpoint under $20^{\circ} \mathrm{C}$ due to the monotropic behaviour of TAGs. In addition to $\beta^{\prime}$ crystals forming directly from the melt, $\alpha$ crystals simultaneously transition into $\beta^{\prime}$ crystals (Fernandes et al., 2013). At endpoint temperatures above $20{ }^{\circ} \mathrm{C}, \beta^{\prime}$ crystals form exclusively from the melt (Litwinenko et al., 2002; De Graef et al., 2006; Foubert et al., 2006). A deep understanding of how processing methods may impact fat crystallization is necessary to industry to maintain final product quality (Herrera and Hartel, 2000; Altimiras et al., 2007; Pérez-Martínez et al., 2007; Afoakwa et al., 2008d; Debaste et al., 2008).

\subsection{Palm Oil in Application}

Confectionery fats may be split into either lauric fats, such as coconut oil and palm kernel oil that are high in lauric acid content, or non-lauric fats, such as CB, PO, and shea butter. While the handling of lauric fats is quite easy, they are very high in saturated fat content and may not be suitable for some applications, such as confectionery fillings. Furthermore, industry has been continuously seeking non-hydrogenated fats to improve the nutritional profile of their products in response to restricted usage of hydrogenated vegetable oils. PO has quickly risen to the top of fat alternatives in industry due to its natural semi-solid 
consistency and affordability, however, its physicochemistry is far lesser known than other fats. Here, the production and chemistry of PO is reviewed.

\subsubsection{Production and Use}

It has been highly recommended to replace saturated fats with poly- and monounsaturated fats to control lipoprotein levels (Hruby and $\mathrm{Hu}, 2016$ ). This is in part due to the association between cardiovascular disease (CVD) and saturated FAs found in animal-based fats (Neaton and Wentworth, 1992), however, positional distribution of these FAs within TAGs must also be considered (Karupaiah and Sundram, 2007). While this generally encourages consumption of plant-based oils, tropical oils are considerably higher in saturation (Edem, 2002; Gee, 2007). This prompted nutritional campaigns in the 1980s which resulted in much

of the present-day aversion to PO by consumers (Allen, 2002; Gratzer, 2005; McNamara, 2010).

Malaysia is currently the largest producer and exporter of PO and palm by-products, followed by Indonesia (Sambanthamurthi et al., 2000). It is cheaper to manufacture than any other plant-based oil (Edem, 2002) and has chemical and thermal properties ideal for applications including a natural semi-solid consistency, relatively slow melting properties, and low foaming properties (Okiy and Oke, 1984; Kheiri, 1987; Cottrell, 1991). Uses of PO and its fractions are listed in Figure 1.8.

$\mathrm{PO}$ is considered a viable substitute for partially hydrogenated oils (PHOs) because of its natural semi-solid consistency and low tendency to become rancid when compared to other plant-based oils (Maache-Rezzoug et al., 1998; Sambanthamurthi et al., 2000; Tarrago-Trani 


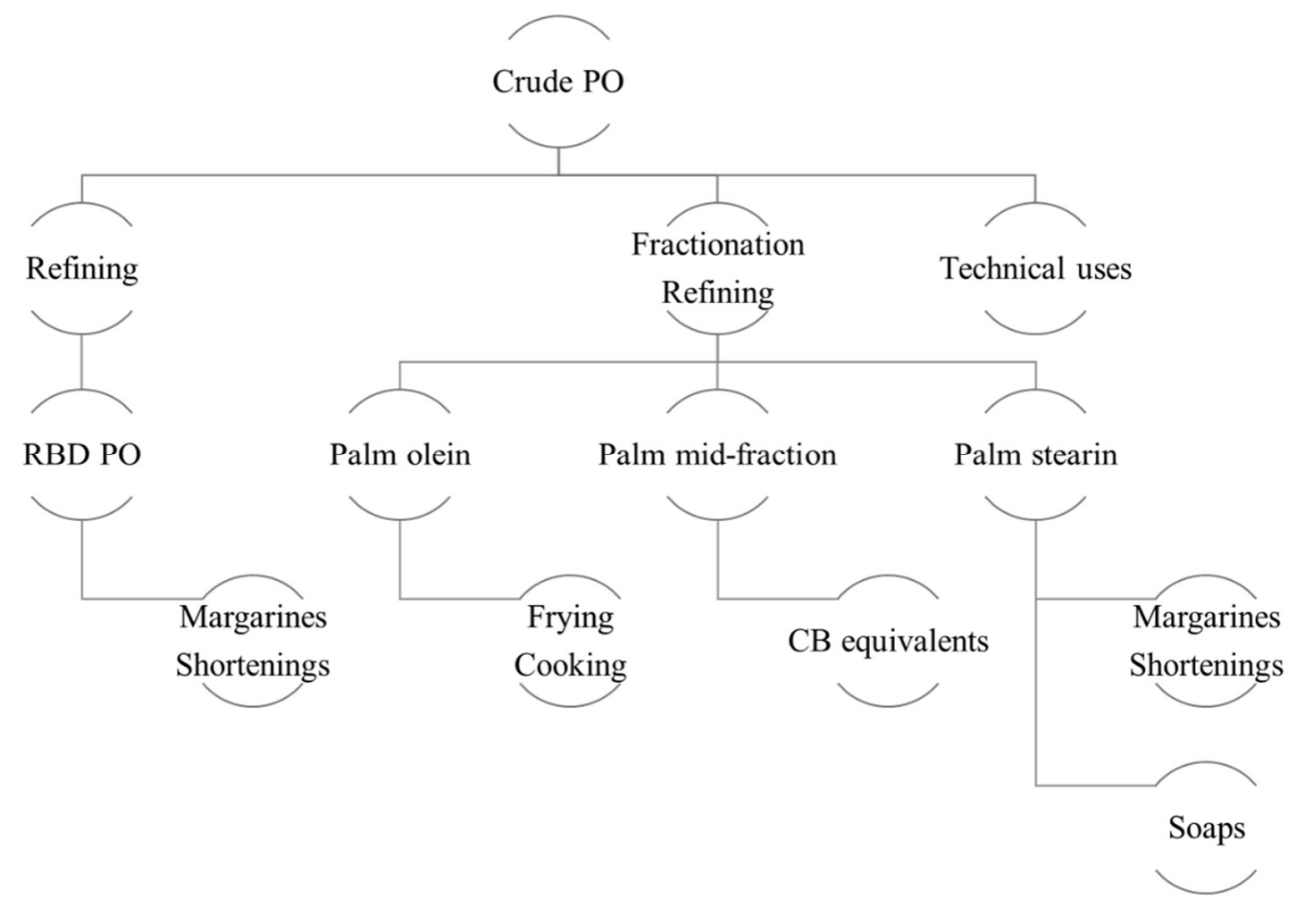

Figure 1.8 Uses of PO and its fractions (adapted from Basiron, 2001).

et al., 2006; Aftab et al., 2013). Several commercial products including biscuits, cream fillings, breads, and meat patties have already been successfully formulated with PO and do not contain the trans fats that are produced from either partial hydrogenation or high temperature deodorization (Fritsche and Steinhart, 1998; Basiron, 2001; Sherazi et al., 2009). Furthermore, consumption of a palm-based diet was recently shown to reduce lowdensity lipoprotein levels (Voon et al., 2011). Despite young consumers seeking fat-reduced options when purchasing food (Do et al., 2008), this has traditionally not been an issue with confections since they are typically consumed in small quantities as treats (Rössner, 1997).

\subsubsection{Composition}

Palmitic and oleic acid are the main FAs found in ripe drupe of oil palm, at 44 and 39-40 \% respectively, followed by linoleic, stearic, and myristic acid (Table 1.4) (Basiron, 2001; Gee, 
Table 1.4 Typical FA composition of PO (Basiron, 2001).

\begin{tabular}{cc}
\hline FA & Composition (\%) \\
\hline Palmitic & 44.0 \\
Oleic & 39.2 \\
Linoleic & 10.1 \\
Stearic & 4.5 \\
Myristic & 1.1 \\
Linolenic & 0.4 \\
Arachidic & 0.4 \\
Lauric & 0.2 \\
\hline
\end{tabular}

2007). To date, no other plant-based oil is found to be as rich in palmitic acid as palm (Sambanthamurthi et al., 2000). TAGs account for over $95 \%$ of PO content with POP being the most abundant (Sambanthamurthi et al., 2000; Gee, 2007). While there exists regioselectivity of unsaturated FAs at the sn-2 position, approximately 7-10 \% of TAGs in PO are trisaturated. Minor components of natural PO include DAGs, MAGs, FFAs, phosphatides, sterols, and trace metals (Siew and Ng, 1995; Sambanthamurthi et al., 2000). The DAG and TAG composition of commercial PO is listed in Table 1.5 and consists mainly of $\mathrm{P}(\mathrm{PO})$ and $\mathrm{O}(\mathrm{OP})$ at 27.6 and $22.5 \%$, respectively.

Carotenoids, e.g., $\beta$-carotene, aid with vision and are responsible for the dark amber colour in raw PO (Edem, 2002). These carotenoids are destroyed after the raw oil has been refined, bleached, and deodorized (Cottrell, 1991). PO is also one of the richest natural sources for tocotrienols (Goh et al., 1985; Sundram and Top, 1994), a subclass of vitamin E that has gained much interest in its role in the circulatory regulation of cholesterol (Sen et al., 2007; Stanley, 2008). 
Table 1.5 DAG and TAG composition of PO. ${ }^{1.3,1.4,1.5}$

\begin{tabular}{cccc}
\hline TAG & PO & TAG & PO \\
\hline P(PO) & 27.56 & $($ LnLO $)$ & 0.02 \\
O(PO) & 22.51 & LaLaLa & 0.02 \\
$(\mathrm{PLO})$ & 10.08 & S(SA) & 0.01 \\
P(PL) & 7.83 & S(SB) & 0.01 \\
PPP & 5.53 & DAG & PO \\
(POS) & 5.23 & $1,3-\mathrm{PO}$ & 1.98 \\
OOO & 4.43 & $1,2-\mathrm{PO}$ & 1.04 \\
O(SO) & 2.55 & $1,3-\mathrm{OO}$ & 0.79 \\
L(PL) & 2.22 & $1,3-\mathrm{PL}$ & 0.70 \\
O(LO) & 2.08 & $1,3-\mathrm{PP}$ & 0.57 \\
P(PS $)$ & 1.09 & $1,2-\mathrm{OO}$ & 0.49 \\
$(\mathrm{PLS})$ & 0.93 & $1,2-\mathrm{PP}$ & 0.25 \\
L(LO) & 0.54 & $1,3-\mathrm{SO}$ & 0.17 \\
(POA) & 0.34 & $1,3-\mathrm{PS}$ & 0.09 \\
L(SL) & 0.27 & $1,2-\mathrm{SO}$ & 0.09 \\
O(AO) & 0.19 & $1,2-\mathrm{PS}$ & 0.05 \\
S(PS) & 0.11 & $1,3-\mathrm{OL}$ & 0.04 \\
$(\mathrm{LnLP})$ & 0.09 & $1,2-\mathrm{OL}$ & 0.01 \\
LLL & 0.05 & $1,3-\mathrm{SS}$ & 0.01 \\
\hline
\end{tabular}

\subsubsection{Processing}

Unrefined PO is relatively unstable and must be processed for industrial use (Dian et al., 2006; Kellens et al., 2007). This instability comes from the relatively high FFA content of PO, i.e., $5 \%(\mathrm{w} / \mathrm{w})(\mathrm{Gee}, 2007$; Murphy, 2007). The mishandling of natural PO, such as exposure to high temperatures, propagates its acidification, darkening, and uncontrolled enzymatic peroxidation (Chong and Sambanthamurthi, 1993; Sambanthamurthi et al., 2000; Edem, 2002). Consumption of these peroxides may lead to health problems including hyperlipidemia, platelet aggregation, and tissue damage (Osim et al., 1992, 1994).

\footnotetext{
${ }^{1.3}$ Method for compositional analysis described in Section 2.2.2.

${ }^{1.4}$ Regioisomers are indicated by fatty acids within parentheses.

${ }^{1.5}$ Arachidic (A), behenic (B), linoleic (L), linolenic (Ln), lauric (La), oleic (O), palmitic (P), stearic (S).
} 
MAGs, FFAs, and other minor components are removed from natural PO following initial processing (Gee, 2007; Kellens et al., 2007). The oil may then be fractionated according to its saturation to produce oils that are functionally optimized for specific applications. For example, higher-melting TAGs can be separated from a melt by selective crystallization using a controlled temperature protocol (Hartel, 2001).

Based on temperature-controlled fractionation, the two main fractions of $\mathrm{PO}$ are palm stearin and palm olein (Tshiamala, 2013). Palm olein melts at 18 to $20{ }^{\circ} \mathrm{C}$ and accounts for twothirds of PO on a weight basis. This liquid fraction is formulated into dressings and cooking oils (Gunstone and Norris, 1983; Stanley, 2008). DAGs co-fractionate into palm olein and produce a eutectic mixture that is responsible for early onset crystallization and clouding (Siew and Ng, 1996a; Gee, 2007; Kellens et al., 2007). Palm stearin composes the remainder of PO and melts at 48 to $50{ }^{\circ} \mathrm{C}$. This fraction is often used in margarines and shortenings (Gunstone and Norris, 1983; Stanley, 2008). The higher-melting TAGs in palm stearin are responsible for its higher crystallization rate compared to palm olein and its ability to form $\beta$ crystals (Che Man et al., 1999). Palm olein and stearin may be further fractionated to produce either mid-fractions used for CB equivalents and other confectionery applications or double-fractionated oils such as super olein, which is used in tocotrienol-rich supplements (Kellens et al., 2007; Stanley, 2008). The physical properties of PO and its fractions are listed in Table 1.6 (Edem, 2002).

Interesterification is another processing method used to treat PO to change its physical properties without changing the FA profile. For example, melting profile, mouthfeel, and spreadability are changed using this technique (Gibon et al., 2007; Kellens 
Table 1.6 Physical properties of PO and its fractions (Edem, 2002).

\begin{tabular}{cccc}
\hline Property & PO & Palm olein & Palm stearin \\
\hline Melting point $\left({ }^{\circ} \mathrm{C}\right)$ & 34.2 & 21.6 & $44.5-56.2$ \\
Relative density & $0.89-0.92$ & $0.91-0.92$ & $0.88-0.89$ \\
$\left(50{ }^{\circ} \mathrm{C} /\right.$ water at $\left.25{ }^{\circ} \mathrm{C}\right)$ & 1.46 & 1.47 & 1.45 \\
Refractive index & 0.10 & 0.10 & $0.00-0.15$ \\
Moisture and impurities $(\%)$ & $47.00-55.83$ & $55.00-61.54$ & $21.60-49.40$ \\
$\quad$ Iodine value & $196.0-208.2$ & $189.0-198.0$ & $193.0-206.0$ \\
Saponification value & $0.010-0.500$ & $0.001-0.500$ & $0.100-1.000$ \\
$\quad\left(\right.$ mg KOH $\left.\cdot \mathrm{g}^{-1}\right)$ & & &
\end{tabular}

et al., 2007). Chemical interesterification completely randomizes the position of FAs along the glycerol skeleton whereas enzymatic interesterification exhibits different degrees of regioselectivity depending on the choice of enzyme (Costales-Rodríguez, et al., 2009). Crossbreeding of the African oil palm with trees naturally lower in saturated content, such as Elaeis oleifera, i.e., American oil palm, has also been pursued to generate PO with lower saturated FA content (Sambanthamurthi et al., 2000).

\subsubsection{Recrystallization}

$\mathrm{PO}$, in addition to other fats and oils, continues to undergo morphological and spatial rearrangement during long-term storage because of recrystallization. The crystalline phase undergoes reconstruction to increase van der Waals interactions and minimize the free energy of the system (Fennema et al., 1973; Hartel, 1998; Russell et al., 1999; Sonoda et al., 2004; Metin and Hartel, 2005). While this is believed to proceed through solid-state transitions, the mechanism is not fully understood and is independent of both temperature and humidity (Fennema et al., 1973; Tshiamala, 2013). It is theorized that recrystallization is driven by either a difference in local equilibria of different sized crystals, i.e., Ostwald 
ripening, the fusion of adjacent crystals, i.e., accretion, or the minimization of crystal surface-to-volume ratios (Kuczynski, 1989; Hartel, 2001).

Recrystallization is of concern to the food industry because of its impact on product quality and shelf life. Common examples of recrystallization include the hardening of fondants and 'freezer burn' of ice cream (Hartel, 2001; Basso et al., 2010). Palm-based products are particularly prone to recrystallization upon storage. For example, margarine may harden, resulting in a gritty product with poor spreadability due to undesired crystal growth (Omar et al., 2005). The tendency for PO to recrystallize is a result of its DAG content causing slow crystallization rates, although this mechanism is not fully understood. MAGs have been added to PO to aid in nucleation and increase the rate of crystallization (Tshiamala, 2013). They tend to form $\alpha$ crystals which readily undergo $\alpha \rightarrow \beta^{\prime}$ transition (Krog, 2001). While phase diagrams have been used to understand and control crystallization in bulk systems, for complex foods, this is quite complicated (Fennema et al., 1973; Hartel, 2001).

Recrystallization of $\mathrm{CB}$ in chocolate, which is responsible for fat bloom, is believed to occur from the growth of pre-existing surface crystals (Jewell, 1972; Timms, 1984; Ziegleder and Moser, 1996; Ziegleder and Schwingshandl, 1998; Tietz and Hartel, 2000; Aguilera et al., 2004; Lonchampt and Hartel, 2004). This may be further promoted by a concentration gradient of TAGs (Lovegren et al., 1976; Talbot, 1994, 1999; Sonwai and Rousseau, 2008; James and Smith, 2009). In addition to $\beta_{2}$ crystals, non-fat ingredients further restrict volumetric flow $(Q)$ of liquid-state TAGs by creating a more tortuous path to the surface (Rousseau, 2007). $Q$ is described by Darcy's law: 


$$
Q=\frac{B * A_{c}}{\eta} * \frac{\Delta P}{L}
$$

where $B$ is the permeability coefficient, $A_{c}$ is the cross-sectional area in which flow occurs, $\Delta P$ is change in the pressure of the system, and $L$ is the distance in which this pressure change takes place (Dibildox-Alvarado et al., 2004; Marangoni and Wesdorp, 2013).

Using porosimetry (Loisel et al., 1997), well- and over-tempered couverture containing 32 $\%(\mathrm{w} / \mathrm{w}) \mathrm{CB}$ was determined to contain pores at 1 and $4 \%(\mathrm{v} / \mathrm{v})$, respectively. These pores are believed to form by contraction upon cooling (Sonwai and Rousseau, 2008). They typically measured $6 \mu \mathrm{m}$ in diameter by $3 \mu \mathrm{m}$ in depth according to atomic force microscopy (AFM) with hundreds being randomly distributed within a square centimetre (Rousseau, 2006). It has been theorized that TAGs migrate through these pores by either diffusion (Miquel et al., 2001; Ghosh et al., 2002; Galdámez et al., 2009), capillary action (Aguilera et al., 2004; Marty et al., 2005), or pressure-dependent convective flow (Dahlenborg et al., 2015).

In the case of pralines where fillings are made of low-melting oils, e.g., triolein-rich hazelnut gianduja, it is theorized recrystallization occurs via two steps. First, as the shell contracts during storage, liquid-state TAGs migrate from the filling to the surface as cone-like wells (Beckett, 2000). Afterward, these structures grow and harden, serving as hosts for $\beta_{1}$ crystals (Smith and Dahlman, 2005; Smith et al., 2007; Sonwai and Rousseau, 2008, 2010; Dahlenborg et al., 2011). The solubilization between shell and filling TAGs disrupts the phase behaviour of the shell and exacerbates recrystallization (Bigalli, 1988; Cebula and Ziegleder, 1993; Couzens and Wille, 1997; Walter and Cornillon, 2002; Dahlenborg, 2014). 
Table 1.7 Effect of temperature cycles on fat bloom formation (Ali et al., 2001). ${ }^{1.6,1.7}$

\begin{tabular}{|c|c|c|c|c|}
\hline \multirow{3}{*}{ Week } & \multicolumn{4}{|c|}{ Storage temperature $\left({ }^{\circ} \mathrm{C}\right)$} \\
\hline & \multicolumn{2}{|c|}{18} & \multicolumn{2}{|c|}{30} \\
\hline & Fat bloom & Cycles & Fat bloom & Cycles \\
\hline 0 & - & 7 & + & 6 \\
\hline 1 & - & 7 & ++ & 4 \\
\hline 2 & - & 7 & ++++ & 2 \\
\hline 3 & - & 7 & ++++ & 1 \\
\hline 4 & - & 7 & ++++ & 1 \\
\hline 5 & - & 7 & ++++ & 1 \\
\hline 6 & - & 7 & ++++ & 1 \\
\hline 7 & - & 7 & ++++ & 1 \\
\hline 8 & - & 7 & ++++ & 1 \\
\hline
\end{tabular}

TAG migration into the shell also lowers its density, causing it to swell, and hardens the filling (Barron, 1977; Minson, 1990; Ziegleder and Moser, 1996; Miquel et al., 2001; Miquel and Hall, 2002; Altimiras et al., 2007). This swelling effect has previously been explored using confocal chromatic displacement sensors (Svanberg et al., 2012). Additional issues in praline fillings with high water activity, e.g., water- or alcohol-based, include sugar bloom and mold growth from improper storage (Minifie, 1989; Larumbe et al., 1991).

While it is believed that storage of CB-based confections at $18{ }^{\circ} \mathrm{C}$ or cooler inhibits solidstate transitions, warmer conditions lead to its rapid onset (Cebula and Ziegleder, 1993; Ali et al., 2001; Lonchampt and Hartel, 2004; Schenk and Peschar, 2004). For example, the recrystallization of well-tempered chocolate into $\beta_{1}$ crystals was observed after 30 days when stored at $30^{\circ} \mathrm{C}$ (Table 1.7) (James and Smith, 2009). Storage temperature fluctuations

\footnotetext{
1.6 -, no bloom; +, weak bloom; ++, bloom; +++, strong bloom; ++++, intensive bloom.

1.7 one cycle is $30^{\circ} \mathrm{C}(16 \mathrm{~h})$ and $20^{\circ} \mathrm{C}(8 \mathrm{~h})$.
} 
may further promote recrystallization (Hachiya et al., 1989), e.g., recrystallization was exacerbated when storage temperatures fluctuated as little as one degree (Hettich, 1966).

MF and its fractions have been shown to inhibit CB recrystallization (Metin, 1997). This was theorized to occur through either slowing down the $\beta_{2} \rightarrow \beta_{1}$ transition by disrupting $\beta_{1}$ lamellar packing (Cook, 1964) or by lowering overall SFC from the eutectic mixture that is formed with CB (Hartel, 1996). Since MF may reduce the SFC via eutectic effects, crystallization rate and crystal size may be reduced as well, potentially softening the product. Higher-melting fractions of MFs may offer a solution to this because they inhibit recrystallization while better preserving SFC (Lohman and Hartel, 1994; Pajin and Jovanovic, 2005). In one study, it was observed that addition of MF at $5.0 \%$ and $7.5 \%$ reduced the presence of wells, however, sensory properties, e.g., firmness, snap, melting, were just maintained at $5.0 \%$ (Sonwai and Rousseau, 2010). MF has been used to prolong the shelf life of confections because of this in addition to its affordability and accordance to the Food and Drug Administration Code of Federal Regulations (Bricknell and Hartel, 1998).

Sugar crystallinity has also been shown to impact fat recrystallization. For example, the appearance of fat bloom was visually reduced in formulations with amorphous sugar compared to crystalline powder sugar. This was attributed to amorphous sugar having a smoother surface, and therefore fewer active sites to facilitate recrystallization. Furthermore, its rounded shape may create a more tortuous, closely packed system in comparison to the randomly shaped particulates of powder sugar, which may ultimately hinder oil migration that is responsible for $\beta_{1}$ crystal growth (Bricknell and Hartel, 1998). 
Other methods used to study recrystallization have included trained sensory panels (Quevedo et al., 2013), whiteness index (Bricknell and Hartel, 1998; Quevedo et al., 2013), area-scale fractal analysis (Brown et al., 1993), computer vision (Briones and Aguilera, 2005), Raman spectroscopy (Dahlenborg et al., 2012), magnetic resonance imaging (Miquel and Hall, 2002), AFM (Hodge and Rousseau, 2002; Rousseau, 2006), optical profilometry (Rousseau et al., 2010), and scanning electron microscopy (James and Smith, 2009; Wang et al., 2010).

\subsection{Characterization of Particle Interactions in Confections}

Fat confectionery texture and crystallization largely depend on the combination of the physical properties of the continuous fat phase, dispersion volume fraction, and, importantly, interactions between the ingredients themselves. For example, the particle size distribution and surface area of sugar as well as the emulsifier type and concentration all impact the processability and sensory attributes of fat-continuous products. Similarly, moisture can have a damaging effect as it causes the clustering of non-fat ingredients, resulting in poor mixing. The characterization of particle interactions is thus critical to the establishment of the factors governing the optimized production of confectionery products. To this day, there remains a significant dearth of information in this regard, owing largely to the lack of appropriate research tools. A nascent research avenue in food science is that of force spectroscopy, which offers the unique prospect of determining the extent of particle interactions in confectionery products rich with non-fat ingredients. 


\subsubsection{Force Spectroscopy}

Scientists have romanced the idea of pulling on both sides of a single chemical bond and measuring the force that is required for bond disruption. Such single-molecule experiments have been possible since the 1990s using a technique known as force spectroscopy (Noy and Friddle, 2013). While not as developed as its imaging ability, AFM-based force spectroscopy can be used to measure both inter- and intramolecular forces (Miles and McMaster, 1995; Morris, 2007; Han and Serry, 2008). AFM force spectroscopy can yield information such as the conformation and elasticity of proteins (Janshoff et al., 2000), intermolecular interactions in biological and synthetic compounds (Mate et al., 1987; Meyer and Amer, 1988; Florin et al., 1994; Noy et al., 1995; Rief, 1997; Li et al., 1999; Ortiz and Hadziioannou, 1999; Merkel et al., 1999; Janshoff et al., 2000; Wang et al., 2002; Zhang and Zhang, 2003), particulate interactions in colloidal systems (Ducker et al., 1991), localized surface interactions (Frisbie et al., 1994), and ligand-receptor binding affinities (Florin et al., 1994). Recently, it was used to assess the impact of emulsifiers on CB colloidal properties (Middendorf et al., 2015, 2016).

AFM, which was developed in 1986 (Binnig et al., 1986), employs a probe that is scanned across a sample where atomic forces between tip and sample are monitored and sample topography can be analyzed (Hodge and Rousseau, 2002; Rousseau, 2007). Cantilever deflection is measured by the 'beam bounce' method where a semiconductor diode laser is reflected off the cantilever back and into a position-sensitive split photodiode detector (Figure 1.9). The electrical signal that is received by the detector is measured in volts and is proportional to deflection of the cantilever (Cappella and Dietler, 1999). 


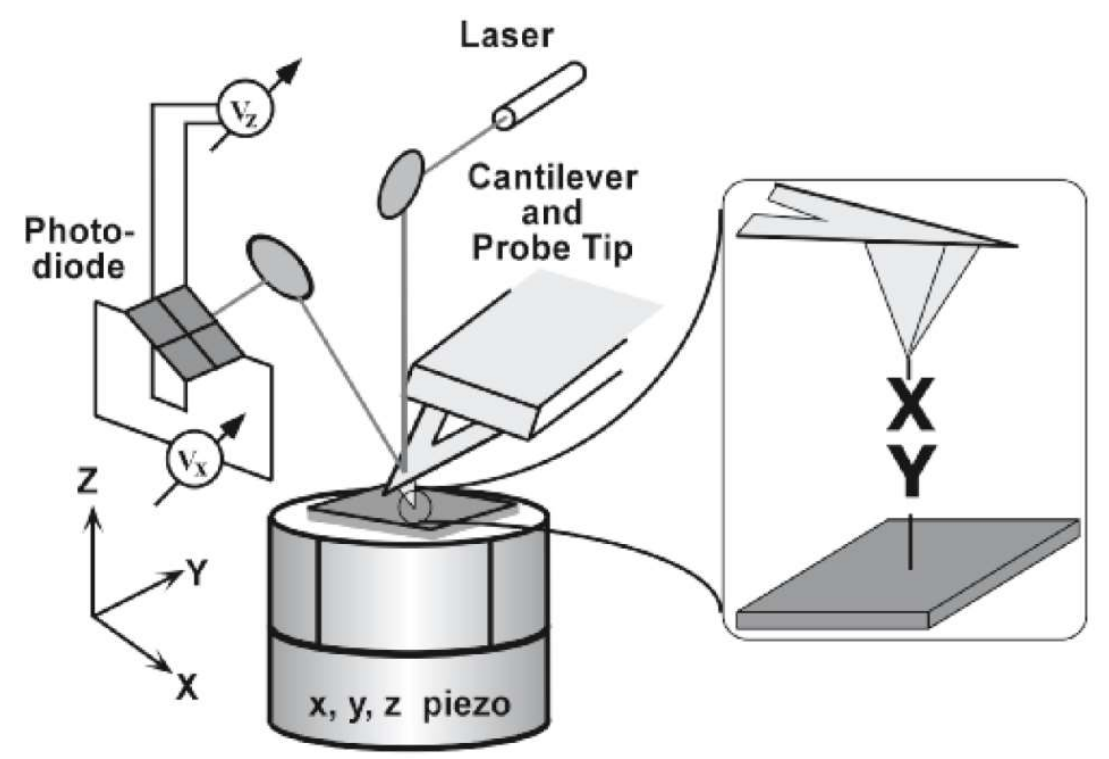

Figure 1.9 Schematic diagram of AFM (Noy et al., 2008).

With AFM-derived force spectroscopy, the tip-sample distance is reduced until 'jump-tocontact' has been achieved. Whereas in 'contact' mode a feedback loop exists to obtain an image, this is notably absent in force spectroscopy (Han and Serry, 2008). The tip-sample distance is then increased and interactive forces are measured. Tip-sample interactions can be repeatedly measured either at the same surface location or at different locations to obtain an interaction map (Owen, 2004). As an alternative to tip retraction, the tip can be intentionally pushed into the sample in a method called nano-indentation (Han and Serry, 2008). Viscoelastic properties typically reserved for rheology, such as Young's modulus, may be obtained. Tip retraction following indentation causes the cantilever to deflect toward the surface because of adhesion (Owen, 2004).

Force spectroscopy is commonly represented as simple curves where the $\mathrm{x}$-axis is the tipsample distance and y-axis is cantilever deflection measured in either volts or Newtons for 


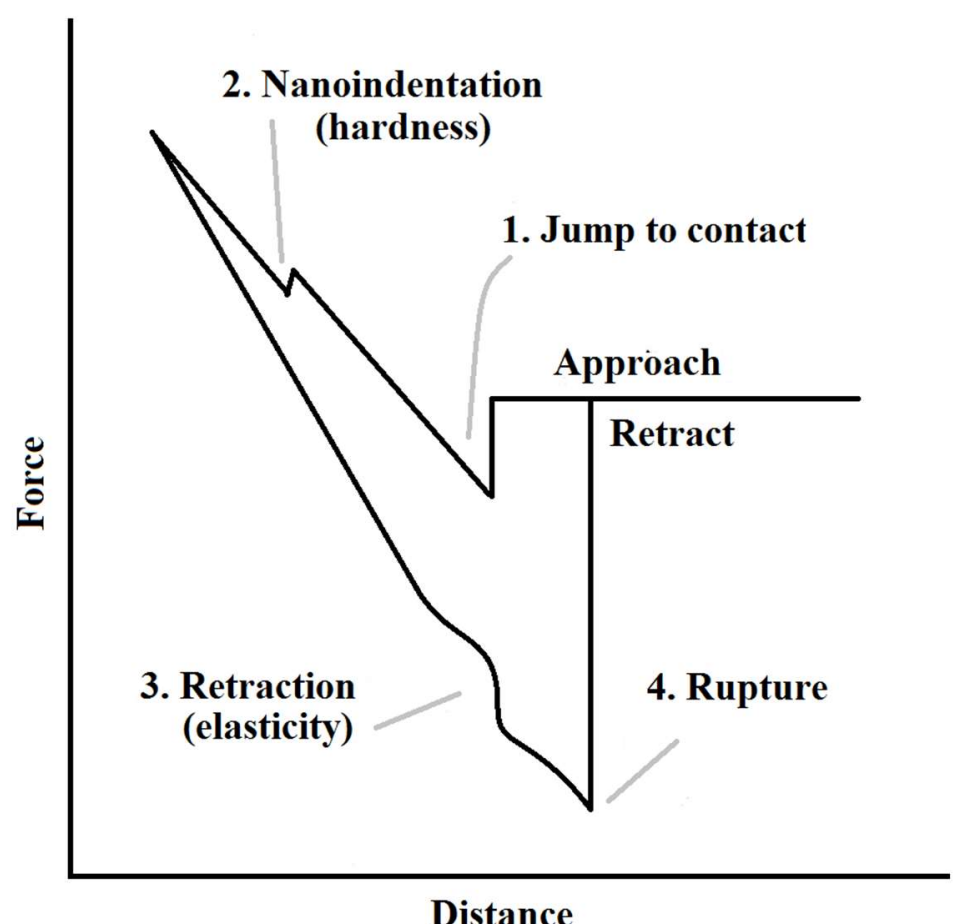

Figure 1.10 Interpretation of force-distance curves (adapted from Kronenberger, 2006).

raw or calibrated signals, respectively. Information that can be extracted from these plots include hardness, elasticity, and rupture bond length (Figure 1.10). These forces may be estimated using the Lennard-Jones potential $(V)$ :

$$
V=4 \varepsilon\left\{\left(\frac{\sigma}{r}\right)^{12}-\left(\frac{\sigma}{r}\right)^{6}\right\}
$$

where $\varepsilon$ is the depth of the potential well, $\sigma$ is the distance when force is zero, and $r$ is the distance of separation, respectively (Seo and Jhe, 2008). To calibrate force with the raw voltage that is detected by the photo diode, the spring constant of the cantilever must first be quantified by one of several methods (Han and Serry, 2008). The first method to derive the spring constant $(k)$ considers the formula: 


$$
k=2 \pi^{3} l^{3} w \sqrt{\frac{\rho^{3}}{E}} f_{0}^{3}
$$

where $l$ is length, $w$ is width, $\rho$ is density, $E$ is Young's modulus, and $f_{0}$ is resonance frequency of the cantilever (Han and Serry, 2008). Another method uses a second cantilever with a pre-calibrated spring constant. When this cantilever is pushed against cantilevers of unknown spring constants, these constants can then be determined (Hinterdorfer et al., 1996). A third method exposes the cantilever to thermal fluctuations, whereby a thermal noise spectrum is obtained and fitted against a Lorentzian function to determine the spring constant (Owen, 2004).

Once the spring constant of the cantilever is known, the deflection distance for a known change in voltage must be determined. This is done by deflecting the cantilever against a clean hard surface. Because of the large force that is exerted on the cantilever, this is often performed post-experiment. After the distance is known, force can be calculated using Hooke's Law:

$$
F=k x
$$

where $x$ is distance determined by the photo diode. Calibration of the cantilever must be performed every time it is mounted to the instrument or between days where conditions such as temperature or humidity differ because deflection may vary. Since deflection values are typically between 0.1 to $100 \mathrm{~nm}$, cantilevers with spring constants between 0.01 and 1.00 $\mathrm{N} \cdot \mathrm{m}^{-1}$ are most ideal for force spectroscopy studies (Noy and Friddle, 2013). 


\subsubsection{Cantilever Functionalization}

The surface of either tip or sample may be intentionally exposed to chemical or biological species to study specific molecular-molecular interactions (Han and Serry, 2008; Tang et al., 2008). In sample functionalization, self-assembling monolayers, e.g., silanes on glass surfaces or thiols on gold surfaces, are formed. In tip functionalization, molecules of interest are often adsorbed from solution. Overlaying the interaction-specific force-distance curves with imaging results in chemical or affinity maps — a technique called volume spectroscopy (Morris, 2007; Han and Serry, 2008).

The easiest way to functionalize tips uses short rigid linker molecules followed by spacers, such as alkyl thiols or short-chained polyethylene glycols, to reduce unwanted tethering. These glycols are often ideal for spacers because they are both chemically and physically inert and allow tethered molecules to reorient (Ebner et al., 2007). Molecules of interest are diluted with the spacer at 2 mol. \% upon which the linker is introduced. An issue in AFM force spectroscopy with functionalized tips is distinguishing specific from non-specific forces with the sample (Noy and Friddle, 2013). Considerations for approaching functionalization in force spectroscopy has been previously reviewed (Blanchette et al., 2008).

Despite little reported on the application of AFM-based force spectroscopy in food science (Middendorf et al., 2015, 2016), biological topics of interest have included the folding and assembling of macromolecules, construction of biomolecular sensors, and virus-host affinity (Han and Serry, 2008). For example, globular proteins exhibit structural anisotropy where 

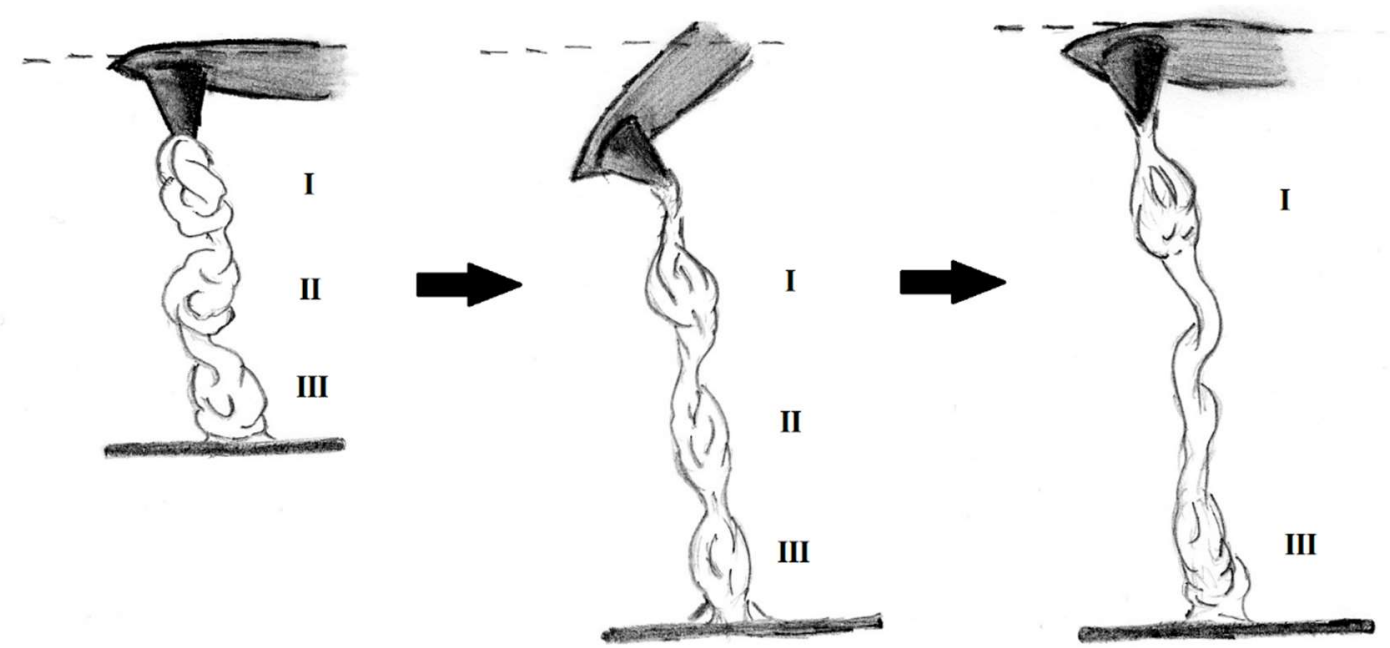

Figure 1.11 Dimension-dependent conformational analysis of a protein depicting the unfolding of conformation II (adapted from Carrion-Vazquez et al., 1999).

the direction of pulling shows unique conformational properties (Figure 1.11) (CarrionVazquez et al., 2003; Dietz et al., 2006; Gao et al., 2011; Graham and Best, 2011; Jagannathan et al., 2012; Ward and Sweeney, 2012). It is suggested that specific fingerprints of unique unfolding events can be obtained, which result from secondary and tertiary conformations (Rief, 1997; Bustamante et al., 2000; Eckel et al., 2003). In another study, graphite demonstrated different stabilities when pulled either parallel or perpendicular to its polycyclic rings (Zheng et al., 2013). Measurements of polysaccharide chains were also studied using AFM-based force spectroscopy (Rief et al., 1997; Zhang and Marszalek, 2006).

\subsection{Conclusions}

The quality of fat-based confections is not only derived from the physical properties of the continuous fat phase, but also its chemistry with the non-fat ingredients contained within. The amount of fat affects the wetting of non-fat ingredients, behaving as a lubricant and 
maintaining proper fluidity, while the non-fat ingredients provide surfaces off of which fat can nucleate but may also change $\eta$ and mobility of the species that contribute to crystal growth. The way in which these ingredients interact strongly influences the path of fat crystallization and ultimately perception of the product by the consumer. Hardness and spreadability are just two such properties that depend heavily on this relationship. An understanding of ingredient interactions is crucial to the success of any product and has become particularly important in confectionery applications, particularly those that are palm-based, where quality is largely determined through trial and error. This opens the door to untapped venues of food science, such as AFM-based force spectroscopy, where specific ingredient-ingredient interactions can be characterized and used to better predict how such systems will crystallize. 


\section{Chapter 2:2.1}

\section{THE EFFECT OF SUGAR ON THE PHYSICAL PROPERTIES OF PALM OIL}

PO is an alternative to PHOs in fat-based confections given its semi-solid consistency and zero trans fat content. However, its slow crystallization behaviour results in hardening during storage and objectionable texture. While there are numerous studies on PO solidification and storage properties in bulk systems, the impact of sugar in this context has yet to be reported. In this chapter, the effect of confectioner's sugar on the crystallization and rheology of PO and a mid-fraction blend were explored over four weeks of storage. PO, which was higher in DAG and saturated TAG content than the mid-fraction blend, was significantly higher in SFC and viscoelasticity over storage. The addition of sugar reduced SFC in both oils but increased their solid-like character, an effect ascribed to the role of the dispersed sugar on oil viscosity. Preliminary principal component analysis on lipid composition identified the key species contributing to these properties. These findings have established a foundation towards understanding the role of sugar as a dispersed ingredient in palm-based confections.

\footnotetext{
2.1 This chapter previously appeared as a published article with following citation: West, R. and Rousseau, D.
} (2016). Crystallization and rheology of palm oil in the presence of sugar. Food Res. Int. 85:224-234. 


\subsection{Introduction}

Obesity is a global epidemic that affects people of all ages and nationalities (Ab Latip et al., 2013). It was reported in 2011 that $67 \%$ of adult males and $54 \%$ of adult females were either overweight or obese in Canada (Statistics Canada, 2012), with 57000 associated deaths between 1985 and 2000 (Katzmarzyk and Ardern, 2004).

Circulatory fat levels are associated with diet and linked to health problems. Trans fats are closely connected with CVD prompting its declaration under Nutrition Facts on Canadian packaging (Ascherio et al., 1999). The suggested limit of trans fats intake is less than $4 \%$ of total fats consumed (Aftab et al., 2013).

Trans fats are formed primarily through partial hydrogenation of polyunsaturated FAs in plant-based oils. The extent of hydrogenation permits control over the crystallization behaviour and consistency of fats, which is important for applications such as snacks and cookies (Yoshikawa et al., 2014). However, health concerns over trans fats have resulted in the re-evaluation and re-formulation of commercial products by many manufacturers. Achieving a zero trans fat label while maintaining quality and brand loyalty is the current industrial challenge in this realm (L'Abbé et al., 2009).

PO is higher in saturated fat compared to other plant-based oils, which has put it in the crosshairs of past nutrition campaigns (McNamara, 2010). Given the recent regulation of trans fats, PO has since returned to many products such as biscuits, breads, and fillings in large part due to its desirable melting profile (Gee, 2007). As well, it is relatively stable against oxidation (Aftab et al., 2013) and higher yielding than any other oil crop (Purseglove, 
1983). New light has also been shed on its effect on biomarkers for disease (Voon et al., 2011).

PO consists of three main types of TAGs: trisaturated [e.g., tripalmitin (PPP)], disaturated [e.g., 1,3-dipalmitoyl-2-oleoyl-glycerol, (POP)], and monosaturated [e.g., 1,2-dioleoyl-3palmitoyl-glycerol (POO)] (Gee, 2007). Appreciable amounts of DAGs and FFAs are also present in unrefined PO (Smith 2001). DAGs have higher slip points than TAGs resulting in waxier mouthfeel that can restrict the application of POs in foods (Ab Latip et al., 2013). DAGs are also responsible for the slow crystallization and post-process hardening of PO although the mechanisms at play remain unknown (Siew and Ng, 1999; Tshiamala, 2013).

Fractionation of PO is used to isolate and increase the concentration of certain TAG groups and other lipids with similar physicochemical properties. The two main fractions of PO are palm stearin and olein, the former being higher in saturated FA content. This increases the crystallization rate of palm stearin, shifting its melting range to higher temperatures (Che Man et al., 1999). DAGs tend to partition into palm olein, which are responsible its eutectic property and clouding during solidification (Gee, 2007). Further fractionation between palm olein and stearin generates palm mid-fractions (PMFs) as shown in Figure 1.8 (Kellens et al., 2007).

Many confectionery products are semi-solid suspensions consisting of fine particles such as sugar crystals dispersed throughout a continuous fat phase. The sensory attributes of such mixtures depend on the size and distribution of these sugar particles as well as the polymorphic state and morphology of the continuous phase. As opposed to simple fats, where TAG crystallization continues unobstructed until a space-filling network is reached, 
the fat crystal spatial distribution and crystallization pathway will be influenced by the presence of this dispersion which can represent upwards of $70 \%(\mathrm{v} / \mathrm{v})$ in commercial products. Dispersed particulates of different sizes can impact properties such as oil migration (Altimiras et al., 2007; Nightingale et al., 2012) as well as the extent and rate of cooling required for fat crystal nucleation and growth (Bowser, 2006; Dhonsi and Stapley, 2006; Sonwai and Rousseau, 2008; Svanberg et al., 2011a and 2011b; Fernandes et al., 2013; Hartel, 2013).

Apart from molten chocolate, few studies have characterized model fat-particulate systems appropriate for confectionery applications. Here, the impact of confectioner's sugar on SFC, rheology, and fat crystal morphology of PO and PMF were characterized over a storage time (t) of four weeks. Ultimately, the information gained from this study is aimed to benefit industry where continuous product reformulations to remove PHOs are underway.

\subsection{Materials and Methods}

\subsubsection{Sample Preparation}

Commercial PO and PMF (IOI Loders Croklaan, Channahon, IL, USA) were used in this study. PMF was blended at $65 \%(w / w)$ with canola oil (CO) (Bunge North America, St. Louis, MS, USA) to obtain similar melting properties as PO according to AOCS Official Method Cd 16b-93 (Figure 2.1) (Firestone, 1998). Oils were processed either as bulk or combined with sieved 6-x confectioner's sugar (Domino Foods Inc., Yonkers, NY, USA) at $50 \%(\mathrm{w} / \mathrm{w})$ using a stand mixer (Hobart Canada Inc., Toronto, ON, Canada). The confectioner's sugar contained $3 \%(\mathrm{w} / \mathrm{w})$ starch, $0.5 \%(\mathrm{w} / \mathrm{w})$ moisture, and possessed an 


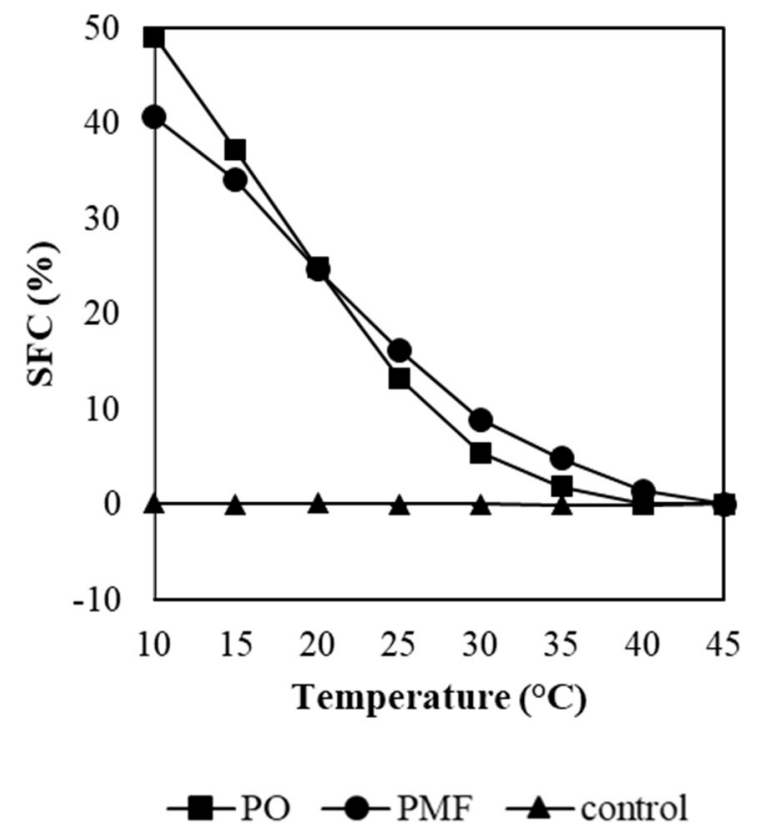

Figure 2.1 Melting profiles of bulk PO, PMF, and control.

average particle size of $30 \mu \mathrm{m}$. CO was used as a control since fat crystallization was not expected in this oil under experimental temperatures (i.e., $20^{\circ} \mathrm{C}$ ).

In a scraped-surface jacketed kettle (Tenon Engineering LTD, Leatherhead, UK) under a constant shear of $100 \mathrm{rpm}$, samples were heated to $60^{\circ} \mathrm{C}$ for $30 \mathrm{~min}$ and then cooled at 1 ${ }^{\circ} \mathrm{C} \cdot \mathrm{min}^{-1}$ to endpoint temperature $20{ }^{\circ} \mathrm{C}$ using a water bath (VWR International 1157P, Mississauga, ON, Canada) equipped with an external probe where they were either analyzed immediately (i.e., $\mathrm{t}=0$ weeks; $<15$ min upon reaching the endpoint temperature) or stored at $20{ }^{\circ} \mathrm{C}$ for weekly analysis (i.e., $\mathrm{t}=1-4$ weeks) to explore the warehouse maturation that many palm-based products undergo before grocer distribution. All samples were made in triplicate. 


\subsubsection{Compositional Analysis}

A high-performance liquid chromatography-mass spectrometry system (Thermo Scientific, Waltham, MA, USA) was used to determine the composition of PO, PMF, and control. The lipids were first separated using liquid chromatography, ionized with coupled chemical ionization, and detected using mass spectrometry. Multiple standards for TAGs, DAGs, and MAGs (Nu-Chek Prep, Elysian, MN, USA) as well as comparison with the Lipid Maps Database (Fahy et al., 2009) were used to confirm identity. It was determined from the chromatographs that these oils contained exclusively TAGs and DAGs. MAGs as well as other lipid components were not detected by this method.

\subsubsection{Pulse Nuclear Magnetic Resonance}

Fat crystallization was monitored by measuring SFC as a function of storage time via pulsed nuclear magnetic resonance (NMR) spectroscopy (Bruker Minispec mq20, Milton, ON, Canada) at $20^{\circ} \mathrm{C}$. Samples were injected into glass NMR tubes (ID $\left.=1 \mathrm{~cm}\right)$ to a height of 4 $\mathrm{cm}$ following cooling and remained in these tubes during storage.

To eliminate false positives contributed by sugar, all SFCs were generated indirectly using AOCS Official Method Cd 16-81 (Firestone, 1998) where the free induction decays (FIDs) of liquid-state protons were exclusively measured. SFC was then determined by the equation:

$$
S F C=100-\left(\frac{R_{60} * S_{20}}{S_{60} * R_{20}}\right) * 100
$$

Sample FID at $20^{\circ} \mathrm{C}\left(S_{20}\right)$ was compared against sample FID at $60{ }^{\circ} \mathrm{C}\left(S_{60}\right)$, where any solid fractions have melted, to determine SFC. CO equilibrated to either $20\left(R_{20}\right)$ or $60{ }^{\circ} \mathrm{C}\left(R_{60}\right)$ 
was used as a reference oil to account for temperature effects on NMR sensitivity. Six subreplicate measurements were made for each NMR tube.

\subsubsection{Differential Scanning Calorimetry}

The total enthalpy of melting $\left(\Delta \mathrm{H}_{\mathrm{T}}\right)$ (i.e., between 30 and $55^{\circ} \mathrm{C}$ ) of oils and oil-sugar blends in open aluminum pans was measured using DSC (Pyris Diamond DSC and v.7 software, Perkin-Elmer Life and Analytical Sciences, Woodbridge, ON, Canada). An empty pan was used as a reference for all runs. Nitrogen gas was used to prevent water condensation. After equilibrating the sample at $20{ }^{\circ} \mathrm{C}$ for $5 \mathrm{~min}$, the temperature was increased to $90{ }^{\circ} \mathrm{C}$ at 5 ${ }^{\circ} \mathrm{C} \cdot \mathrm{min}^{-1}$. Values in the oil-sugar blends were corrected for fat content.

\subsubsection{Rheology}

Viscoelasticity was measured with a controlled strain rheometer (AntonPaar Physica MCR301, St. Laurent, QC, Canada). The objective for rheology was two-fold with the first being the assessment of fat crystallization during the first 20 min post-processing (Nusantoro, 2009). The linear viscoelastic region, which measures material properties without imposing instrumental destruction, was determined to exist mutually at $1 \mathrm{~Hz}$ frequency with $0.01 \%$ strain across all sample compositions. A serrated PP25/P2 geometry was used to minimize

slippage. Rheograms were generated by plotting mean phase angle $(\delta)$ and complex modulus $\left(\left|G^{*}\right|\right)$ against time. The second objective was to characterize storage modulus $\left(\mathrm{G}^{\prime}\right)$, loss moduli $\left(G^{\prime \prime}\right)$, and complex viscosity $\left(\eta^{*}\right)$ to determine the effects of sugar and storage (Omar et al., 2005). This was achieved by performing frequency sweep tests at $0.01 \%$ strain [i.e., 
21 points of angular frequency $(\omega)$ were collected between 4 and $\left.63 \mathrm{rad} \cdot \mathrm{s}^{-1}\right]^{2.2}$ All rheological tests were performed at $20^{\circ} \mathrm{C}$ with a gap size of $1.0 \mathrm{~mm}$.

\subsubsection{Microscopy}

Brightfield microscopy (Axiovert 200M Inverted Microscope Zeiss, Toronto, ON, Canada) was used to identify fat crystals in the samples. A temperature of $20{ }^{\circ} \mathrm{C}$ was maintained using a Peltier-controlled temperature stage (model TSA02i with STC200 controller, Instec, Boulder, CO, USA). Imaging software ImageJ 1.48V (National Institutes of Health, USA) was used to assess crystal morphology and size.

\subsubsection{Statistical Analysis}

A four-way analysis of variance (ANOVA) from a mixed model using replicate, oil, addition of sugar, and storage time as variables (plus interactions with storage) was performed to test for significance $(\mathrm{p}<0.05)$ using SAS 9.4 (SAS Institute, Cary, NC, USA). ${ }^{2.3}$ Experimentspecific variables were used as needed. Insignificant interaction variables were removed from these models and their degrees of freedom added back into error. Principal component analysis (PCA) was used to associate composition (i.e., acylglycerol and sugar) to the properties measured in this study.

2.2 Raw frequency sweep tests for $\mathrm{G}^{\prime}$ are provided in $\mathrm{A} 1$.

${ }^{2.3}$ ANOVA tables are listed in A2.1. 


\subsection{Results and Discussion}

\subsubsection{Solid Fat Content and Enthalpy of Melting}

With the SFCs for all samples being non-zero at 0 weeks (Figure 2.2), this meant that earlystage crystallization occurred during processing as samples were cooled from 60 to $20{ }^{\circ} \mathrm{C}$. Immediately following cooling, SFC values for PO and PMF were $13.2 \pm 0.9$ and $14.3 \pm 0.6$ $\%$ in bulk and $17.3 \pm 0.8$ and $9.6 \pm 1.7 \%$ in oil-sugar blends, respectively. DAGs such as dipalmitoylglycerol (e.g., PP) can enhance crystallization of palm fractions while others can inhibit it (Siew and $\mathrm{Ng}, 1996 \mathrm{~b}$ ). The greater SFC for PO may have resulted from the combination of its higher DAG as well as mono- and disaturated TAG contents which promoted crystallization (Table 2.1) (Siew and Ng, 1996a; Gee, 2007; Tshiamala, 2013). The difference in SFC between oils (i.e., average across sugar) increased with storage as well. The total unsaturated TAG content, which is low-melting and does not contribute towards crystallization, was over four-fold higher in PMF compared to PO. This in addition to its lower DAG was likely responsible for the lower SFC observed in PMF.

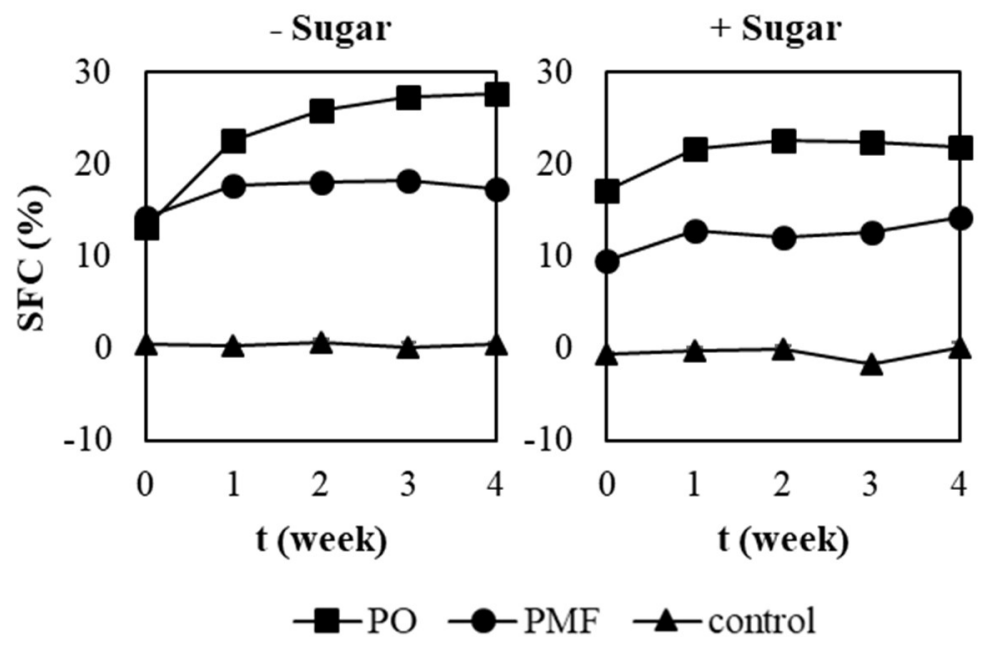

Figure 2.2 Effect of sugar on SFC of PO and PMF over time. 
Table 2.1 Acylglycerol composition of PO, PMF, and control. ${ }^{2.4,2.5}$

\begin{tabular}{|c|c|c|c|c|c|c|c|}
\hline TAG & PO & PMF & Control & TAG & PO & PMF & Control \\
\hline $\mathrm{P}(\mathrm{PO})$ & 27.56 & 23.40 & 0.25 & $\mathrm{~S}(\mathrm{SB})$ & 0.01 & 0.01 & 0.00 \\
\hline $\mathrm{O}(\mathrm{PO})$ & 22.51 & 13.12 & 5.68 & SSS & 0.00 & 0.01 & 0.00 \\
\hline (PLO) & 10.08 & 5.78 & 3.97 & $\operatorname{Ln}(\mathrm{LnL})$ & 0.00 & 0.52 & 1.48 \\
\hline $\mathrm{P}(\mathrm{PL})$ & 7.83 & 4.75 & 0.23 & L(LnL) & 0.00 & 0.26 & 0.75 \\
\hline PPP & 5.53 & 5.42 & 0.00 & $\operatorname{Ln}(\mathrm{LnO})$ & 0.00 & 0.08 & 0.23 \\
\hline (POS) & 5.23 & 4.24 & 0.22 & $\mathrm{~S}(\mathrm{SO})$ & 0.00 & 0.08 & 0.07 \\
\hline $\mathrm{OOO}$ & 4.43 & 13.77 & 33.73 & & & & \\
\hline $\mathrm{O}(\mathrm{SO})$ & 2.55 & 2.15 & 2.68 & DAG & PO & PMF & Control \\
\hline $\mathrm{L}(\mathrm{PL})$ & 2.22 & 1.28 & 0.98 & $1,3-\mathrm{PO}$ & 1.98 & 0.96 & 0.09 \\
\hline $\mathrm{O}(\mathrm{LO})$ & 2.08 & 10.33 & 27.05 & 1,2-PO & 1.04 & 0.58 & 0.02 \\
\hline $\mathrm{P}(\mathrm{PS})$ & 1.09 & 1.64 & 0.00 & $1,3-\mathrm{OO}$ & 0.79 & 0.56 & 0.57 \\
\hline (PLS) & 0.93 & 0.65 & 0.00 & 1,3-PL & 0.70 & 0.31 & 0.07 \\
\hline L(LO) & 0.54 & 3.63 & 9.78 & 1,3-PP & 0.57 & 0.53 & 0.00 \\
\hline (POA) & 0.34 & 0.27 & 0.04 & $1,2-\mathrm{OO}$ & 0.49 & 0.33 & 0.25 \\
\hline L(SL) & 0.27 & 0.10 & 0.00 & 1,2-PP & 0.25 & 0.33 & 0.00 \\
\hline $\mathrm{O}(\mathrm{AO})$ & 0.19 & 0.36 & 0.77 & $1,3-\mathrm{SO}$ & 0.17 & 0.09 & 0.03 \\
\hline $\mathrm{S}(\mathrm{PS})$ & 0.11 & 0.23 & 0.00 & 1,3-PS & 0.09 & 0.09 & 0.00 \\
\hline (LnLP) & 0.09 & 0.42 & 1.09 & $1,2-\mathrm{SO}$ & 0.09 & 0.05 & 0.01 \\
\hline LLL & 0.05 & 0.49 & 1.34 & 1,2-PS & 0.05 & 0.06 & 0.00 \\
\hline (LnLO) & 0.02 & 3.01 & 8.57 & 1,3-OL & 0.04 & 0.03 & 0.03 \\
\hline LaLaLa & 0.02 & 0.02 & 0.00 & 1,2-OL & 0.01 & 0.01 & 0.01 \\
\hline $\mathrm{S}(\mathrm{SA})$ & 0.01 & 0.03 & 0.00 & $1,3-\mathrm{SS}$ & 0.01 & 0.01 & 0.00 \\
\hline
\end{tabular}

${ }^{2.4}$ Regioisomers are indicated by fatty acids within parentheses.

${ }^{2.5}$ Arachidic (A), behenic (B), linoleic (L), linolenic (Ln), lauric (La), oleic (O), palmitic (P), stearic (S). 


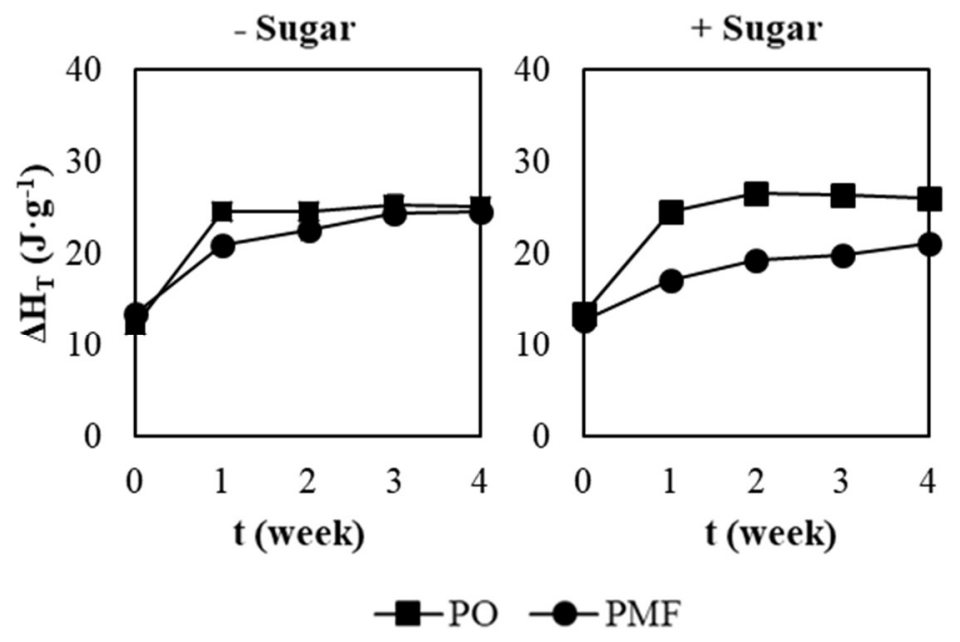

Figure 2.3 Effect of sugar on $\triangle H_{T}$ of $P O$ and $P M F$ over time.

Crystallization is notoriously slow in PO often leading to fractionation and hardening during storage (Smith et al., 2011). Once SFC approaches a plateau, equilibrium is thought to be reached (Toro-Vazquez et al., 2002). The SFC plateau of bulk PMF was observed after one week of storage but was not observed within the four weeks of analysis for bulk PO. DAGs, whose total content in PO was 1.6 times higher than in PMF, are believed to inhibit nucleation and slow crystal growth, though any mechanism remains to be elucidated (Siew and $\mathrm{Ng}, 1999$ ). The mechanism by which DAGs delayed crystallization in the bulk oils appeared different from the oil-sugar blends given the different SFC curves with respect to storage.

$\Delta \mathrm{H}_{\mathrm{T}}$ was higher in the bulk oils compared to their corresponding oil-sugar blends (Figure 2.3). All samples experienced gradually higher $\Delta \mathrm{H}_{\mathrm{T}}$ over storage (e.g., nearly two-fold higher for bulk PO and three-fold higher for bulk PMF at week 4 compared to week 0). While it was determined that the interaction for $\Delta \mathrm{H}_{\mathrm{T}}$ between oil type and sugar as a function of storage was insignificant (Figure 2.3), fixing the effect of sugar revealed a significantly 
higher $\Delta \mathrm{H}_{\mathrm{T}}$ for PO, most likely due to its greater DAG and saturated TAG content. No endotherm was reported for the control and its $\Delta \mathrm{H}_{\mathrm{T}}$ was therefore omitted from Figure 2.3.

$\Delta \mathrm{H}_{\mathrm{T}}$ appeared to be significantly reduced by sugar when the effect of oil type was fixed, suggesting crystallization inhibition by the sugar (Figure 2.3), which corroborated the SFC results (Figure 2.2). While fixing the effect of $\mathrm{PO}$ did not yield significance, this reasoning was due to the average $\Delta \mathrm{H}_{\mathrm{T}}$ being significantly higher at every point in time. In fact, the main effect of sugar itself where both oil type and storage were fixed was significant for $\Delta \mathrm{H}_{\mathrm{T}}$.

\subsubsection{Viscoelasticity}

The texture of fat-based confections is affected by the properties of the fat crystal network, whose rheology is a collective product of SFC, microstructure, and ingredient interactions (Van Den Tempel, 1979). Both PO and PMF showed an increased $\left|\mathrm{G}^{*}\right|$ and decreased $\delta$ as time elapsed, quickly exhibiting more elastic-like properties and suggesting the formation of rigid crystal networks (Tshiamala, 2013), in both bulk and oil-sugar blends (Figure 2.4).

Such rheograms typically show the intersection of $\left|\mathrm{G}^{*}\right|$ and $\delta$. In the present study, however, this intersection must have occurred during the cooling stage of sample preparation and, therefore, could not be observed by the rheometer. As well, PO characteristically undergoes two-step crystallization at temperatures under $20{ }^{\circ} \mathrm{C}$ due to the formation and evolution of different fat crystal polymorphs (Tshiamala, 2013). As experiments were run at $20^{\circ} \mathrm{C}$, this

was not observed. The change in $\left|\mathrm{G}^{*}\right|$ over time was smaller for the oil-sugar blends given 


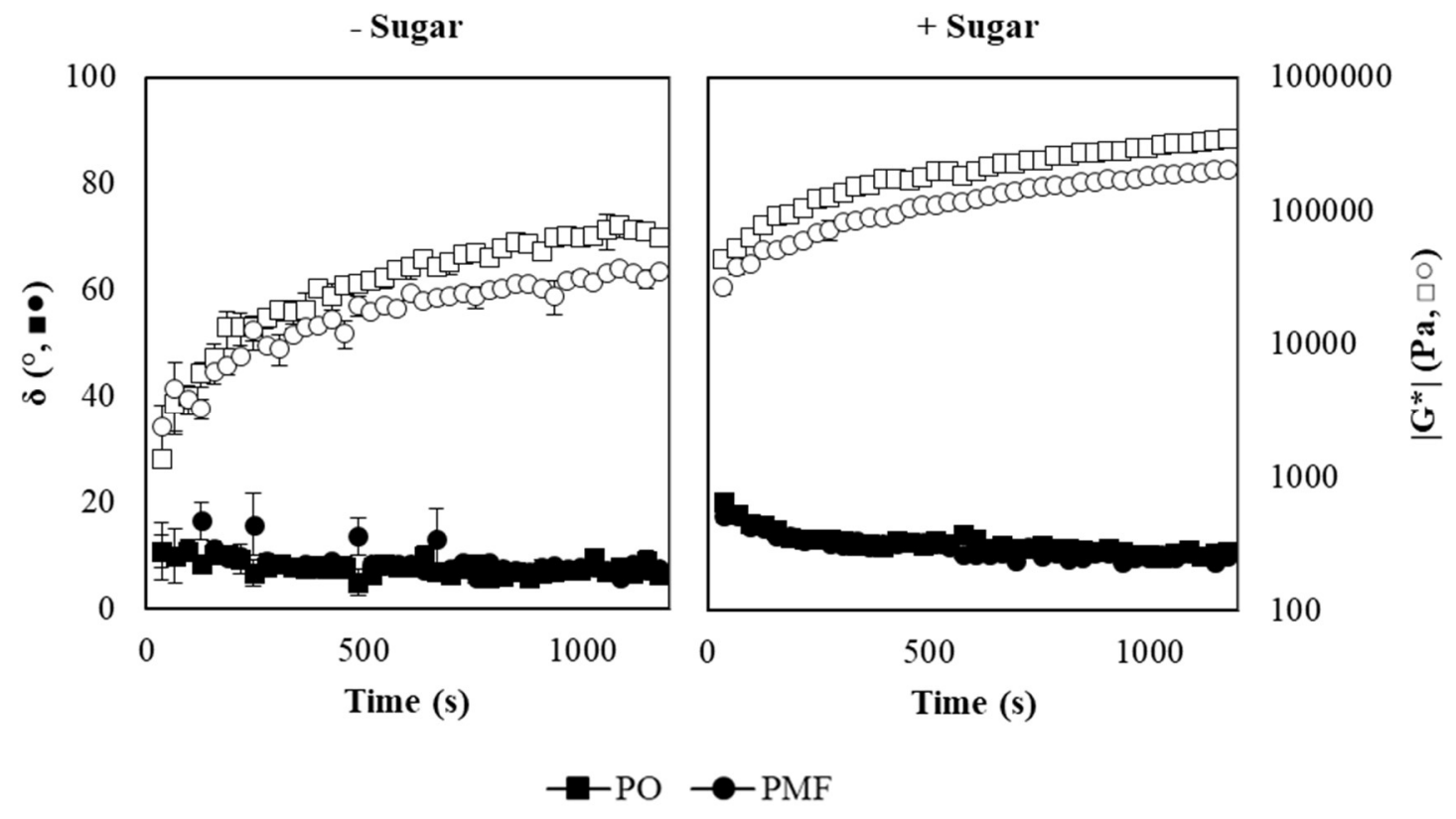

Figure 2.4 Effect of sugar on $\left|G^{*}\right|$ and $\delta$ of $P O$ and PMF immediately after processing.

their projected left-shifted intersection, inferring that crystallization was possibly further advanced in the oil-sugar blends due to sugar-induced fat nucleation. Alternatively, sugar could have contributed through another interaction effect, e.g., the increased $\eta^{*}$ of the oil phase (further explained in Section 2.3.5).

Frequency sweep tests revealed significant interactions between oils and sugar as a function of storage for $\mathrm{G}^{\prime}$ (Figure 2.5 ) and $\mathrm{G}^{\prime \prime}$ (Figure 2.6). Although very similar immediately after processing, $\mathrm{G}^{\prime}$ and $\mathrm{G}^{\prime \prime}$ differences between the oils increased markedly with PO having higher greater viscoelasticity than PMF over time. Despite maximum SFCs being reached during storage in the PO-sugar blend (Figure 2.2), there was no observable plateau in $\mathrm{G}^{\prime}$ and $\mathrm{G}^{\prime \prime}$. This suggested the possibility of continued network ripening, and hardening during storage. Whereas simple fat crystal growth may lead to plateau in viscoelasticity upon 


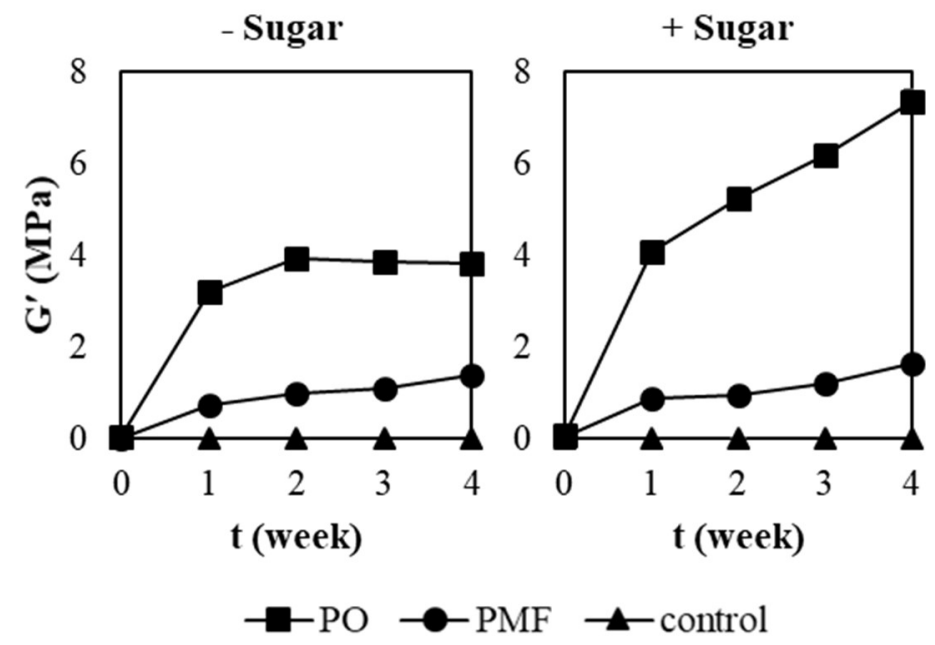

Figure 2.5 Effect of sugar on $G^{\prime}$ of $P O$ and $P M F$ over time.

storage, Ostwald ripening may result in the hardening and change in rheological properties (Coutinho et al., 2003). In our case, this was not measurable via pulsed NMR as the solidliquid ratio did not change with time. In this regard, SFC measurements may be an insensitive marker of changes in rheological properties for these systems.

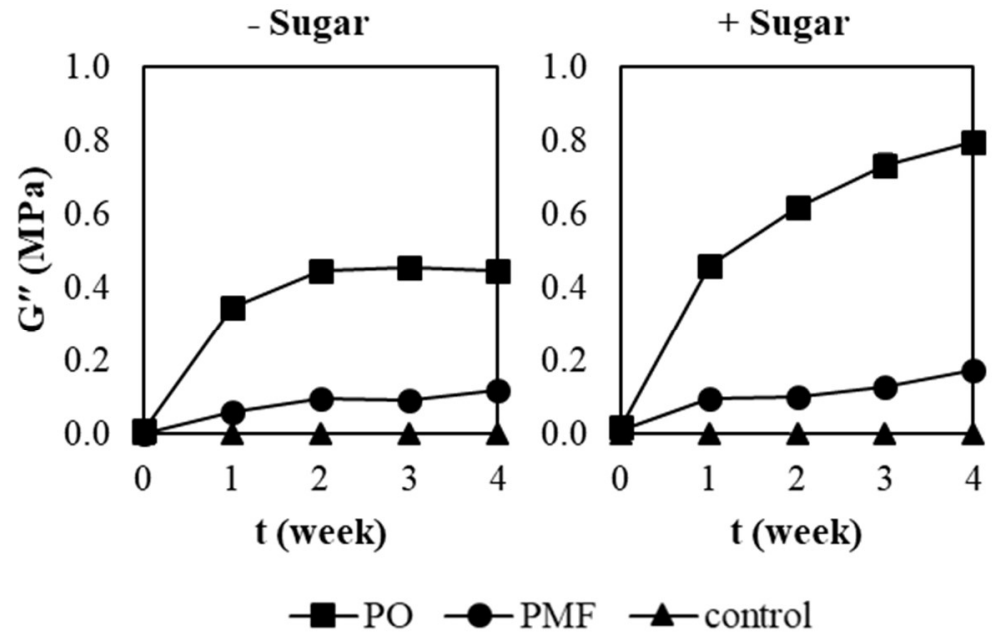

Figure 2.6 Effect of sugar on $G^{\prime \prime}$ of $P O$ and PMF over time. 
Finally, the addition of sugar significantly increased both the $G^{\prime}$ and $G^{\prime \prime}$ of the fat-sugar composites as a function of storage when the effect of oil type was fixed, a trend inverse to that of SFC (Figure 2.2). The viscoelastic properties of the control oil-sugar blend (e.g. baseline) confirmed that observable changes in rheology upon storage were exclusively from oil and not due to the presence of sugar. The increase in these viscoelastic properties in oilsugar blends for both POs suggested a tighter, more rigid fat crystal network.

\subsubsection{Fat Crystal Morphology}

Fat crystals were observed in both bulk PO and PMF (Figure 2.7) immediately following cooling (i.e., week 0) as spheroids of 50-100 $\mu \mathrm{m}$ diameter, with the surface of these crystals appearing smoother in the latter. In PO, the fat crystals continued to grow during storage (i.e., $>100 \mu \mathrm{m}$ diameter at week 1) and there was obvious crystal network growth. By contrast, both fat crystal and network growth in PMF were more limited, given the presence of liquid fat within the continuous phase. Since amount of fat crystals and their size can affect rheology (Van Den Tempel, 1979), the observed smaller, smoother crystals and lessdeveloped network of PMF were likely responsible for the reduced $\mathrm{G}^{\prime}$ and $\mathrm{G}^{\prime \prime}$ values. Lower SFC values further reaffirmed the observed fat crystal morphology, as there was more liquid fat in comparison to PO.

In the oil-sugar blends, sugar particles and starch granules were suspended in liquid fat for both PO and PMF regardless of storage time (Figure 2.8). At no point during the study were individual fat crystals or a network visible, despite oil-sugar blends having measurable SFCs at all times of measurement and exhibiting solid-like character with handling. As noted 


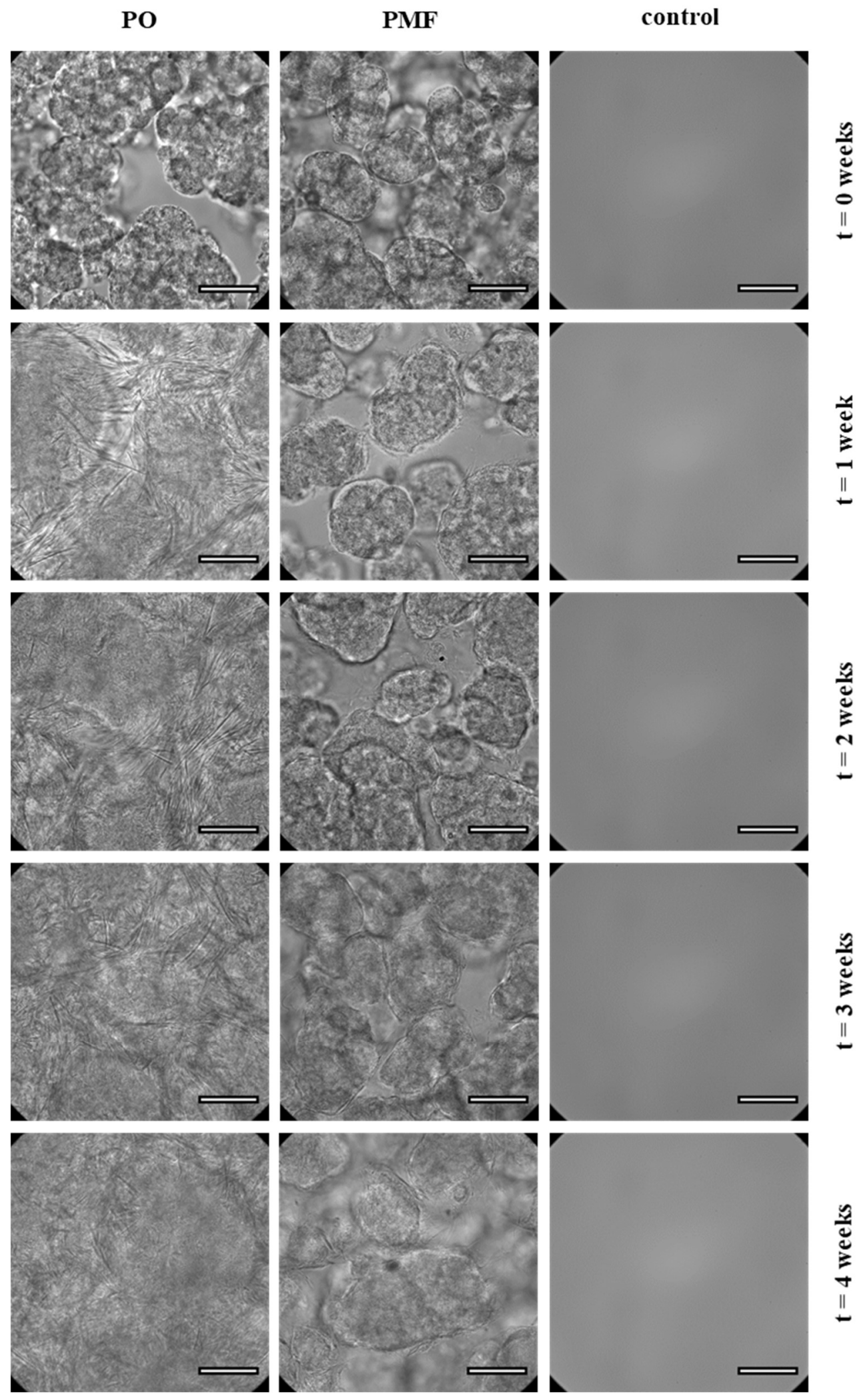

Figure 2.7 Fat crystal morphology of bulk PO and PMF over time (bar $=50 \mu \mathrm{m})$. 


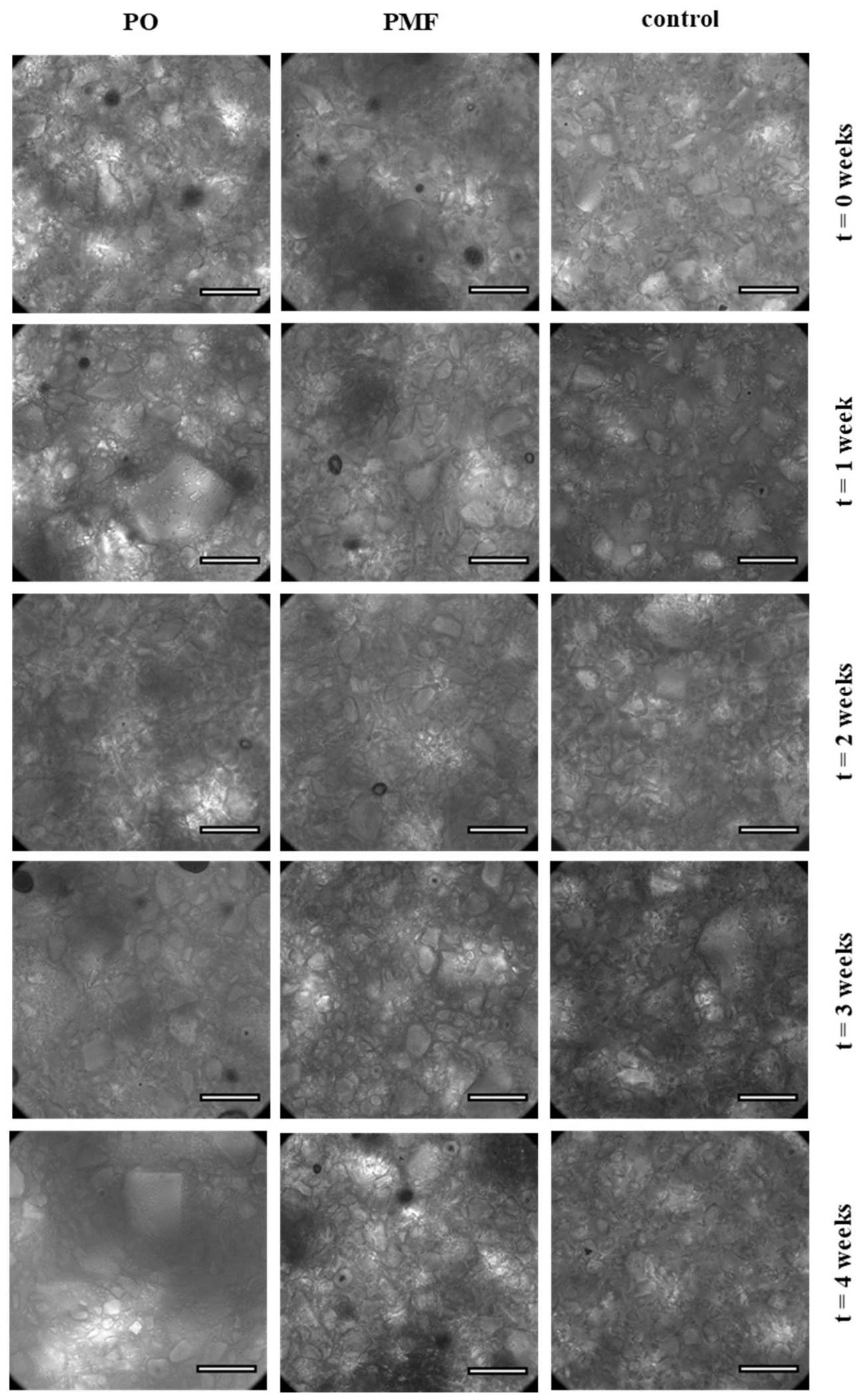

Figure 2.8 Fat crystal morphology of $P O$ - and $P M F$-sugar blends over time (bar $=50 \mu \mathrm{m})$. 
previously, if nucleation occurs too quickly, many crystals can form but will be very small in size (Hartel, 2001). It is quite likely that the facilitation of nucleation by confectionery sugar produced fat crystals smaller than what can be resolved by light microscopy. The averaging of out-of-focus planes from light microscopy may have masked such crystals, prompting consideration for higher resolution techniques that correct for this effect. Attempts with polarized light microscopy did not offer any advantage over brightfield microscopy.

\subsubsection{Composition}

Both DAG and TAG compositions are reported in Table 2.1 . Nearly $60 \%$ of PO was composed of palmitic-rich TAGs $\mathrm{P}(\mathrm{PO})$ (27.6 \%, disaturated), $\mathrm{O}(\mathrm{PO}) \quad(22.5 \%$, monosaturated), and (POL) (10.1\%, monosaturated). The TAGs P(PO) (23.4 \%), OOO (13.8 \%, unsaturated), $\mathrm{O}(\mathrm{PO})(13.1 \%)$, and $\mathrm{O}(\mathrm{LO})(10.3 \%$, unsaturated) composed $\sim 60 \%$ of PMF. Total DAG compositions were 6.4 and $4.0 \%$ for PO and PMF, respectively. The combination of both higher saturated content and DAG content for PO may have explained its consistently larger SFCs and viscoelasticity compared to PMF. The control (i.e., CO) was composed of oleic and linoleic-rich TAGs [OOO (33.7 \%), O(LO) (27.1\%), L(LO) (9.8\%, unsaturated)] and had a total DAG content of $1.1 \%$.

A PCA biplot of the data (Figure 2.9) was constructed and explained $\sim 86 \%$ of all variance. The first component ( $\mathrm{PC1}, 66 \%$ ) separated the continuous phase based on saturation/meltability. Apart from minor TAG (LnLP), all negative values of this component consisted of fully unsaturated TAGs. Likewise, all positive values either corresponded to 


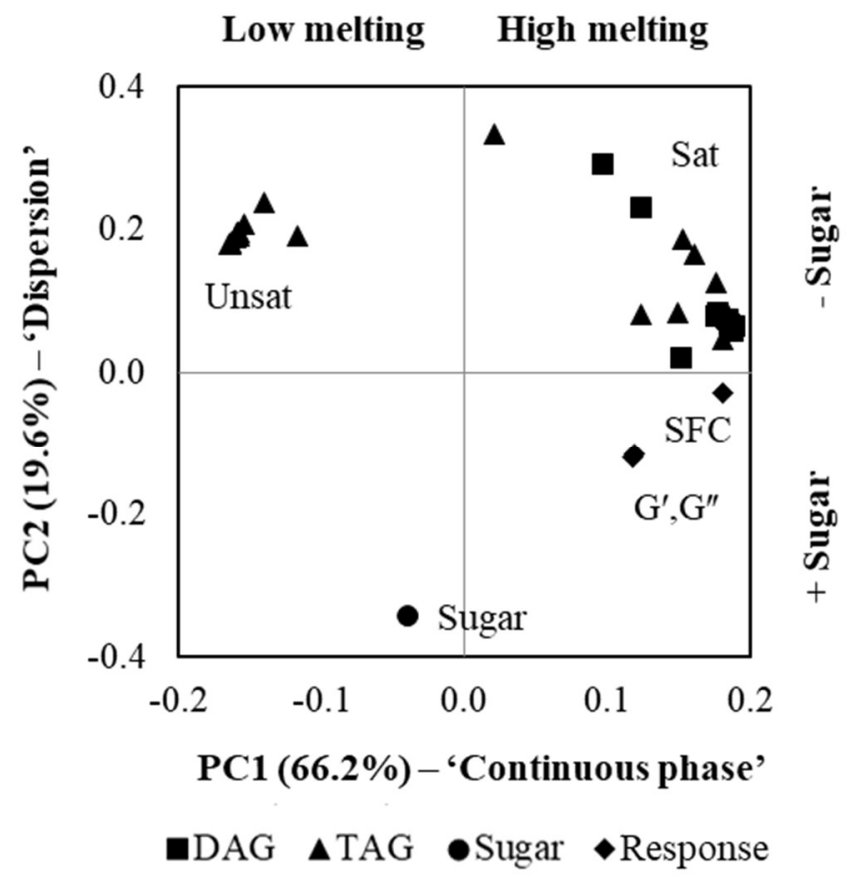

Figure 2.9 PCA biplot of 'continuous phase' vs. 'dispersion'.

DAGs or TAGs containing at least one saturated FA, except for O(LO) and OOO. Both SFC and viscoelastic properties were closely associated with saturated TAGs and DAGs whereas unsaturated TAGs had an opposite effect. The second component (PC2, $20 \%$ ) was mostly weighted by the effect of confectioner's sugar where positive values correspond to its absence (i.e., bulk oils) and negative values corresponded to its presence (i.e., oil-sugar blends). While there was some association for higher viscoelastic properties in the presence of sugar, SFC remained closer to the axis of PC2 as it was observed to be suppressed by addition of sugar (Figure 2.2).

\subsubsection{Mechanistic Considerations}

The oil-sugar blends consisted of the matrix oil phase as the continuous network and the sugar particles as the dispersion embedded within this matrix. The presence of sugar significantly changed the rheology as well as the rate of crystallization of the continuous 
phase oil. Using brightfield microscopy, very few fat crystals were visible until after one week of solidification, suggesting hindrance of fat crystal growth or a change in crystal morphology resulting in lack of visibility using this technique. However, pulsed NMR and DSC clearly confirmed the presence of solid fat crystals within these oil-sugar blends. This contrasted with the bulk oils where crystals readily appeared within minutes when sufficient supercooling was applied.

In bulk oil, crystal growth originates from the first crystallites that form, which will be limited kinetically to those that are most readily nucleated (i.e., the highest-melting TAGs/DAGs). Rapid growth favours the creation and likely persistence of such crystals, while slower growth would give time for transformations into more stable polymorphs, in accordance with the Ostwald step rule (i.e., the earliest formed phases are those most easily nucleated rather than the most stable).

Bulk oil crystallization occurs via heterogeneous nucleation due to the presence of impurities. When present in sufficiently high concentrations, both nucleation and growth are influenced by dispersed particulates such as surfactants, higher-melting TAGs, or foreign material (e.g., dust). Sugar crystals have been found to act as nucleation sites during fat crystallization. Dhonsi and Stapley (2006) suggested that CB heterogeneous nucleation on the surface of sugar crystals resulted in the formation of less stable fat crystal polymorphs. Svanberg et al. (2011b) found that sugar reduced the induction time for CB nucleation, but only if it had been improperly pre-crystallized. Notably, as per our results, in the absence of any emulsifier these authors found no microscopy evidence of the sugar surface catalyzing heterogeneous nucleation. However, as with this study, we did not differentiate sugar crystals based on size, which clearly warrants further investigation. 
Inhibition in the presence of additives has mostly been attributed to their adsorption onto the active growth sites of crystal surfaces, with accessibility to such sites dependent on impurity concentration. However, given the absence of added emulsifier and apparent lack of interaction between the sugar crystal surface and the surrounding TAG milieu, another mechanism was responsible for the apparent inhibition herein observed.

One possibility was the significant increase in continuous phase $\eta$ due to the presence of the dispersed sugar crystals. In industrial crystallization processes (e.g., in concentrated sugar solutions), mass transport will reduce crystal growth rates if a significant increase in $\eta$ occurs leading to a decrease in molecular diffusivity. In lipid systems, it has been argued that this is unlikely as only moderate supercooling is typically applied (Walstra et al., 2001). For instance, the $\eta$ of many vegetable oils increases by a factor of $\sim 3$ upon cooling from 60 to $25^{\circ} \mathrm{C}$ (Santos et al., 2005), which suggests that $\eta$ prior to crystallization onset plays a minimal role. Though it is well-known that the $\eta$ of a fat-oil blend will greatly increase with the presence of fat crystals in the continuous oil phase, separating the role of the melt $\eta$ on TAG crystallization is not trivial as the melt is also the feedstock for the TAGs undergoing crystallization.

Nucleation rate depends on both $\Delta G_{c}$ and $\Delta G^{\prime}{ }_{v}$ for the incorporation of new molecules into a crystal embryo, as was described previously in Equation 1.4 (Turnbull and Fisher, 1949). As supercooling is increased, $\Delta G_{c}$ decreases resulting in a faster rate of nucleation. However, there are barriers to nucleation as molecules must diffuse from the melt to the nucleus site and adopt a configuration appropriate for adsorption onto the surface of a growing nucleus. Under certain circumstances, molecular diffusion will dominate over nucleation and the rate of nucleation will decelerate. It has been shown that $\Delta G^{\prime}{ }_{v}$ is a function of $\eta$ : 


$$
\Delta G_{v}^{\prime}=k T * \ln \left(\frac{3 \pi v \lambda^{3} \eta}{k T}\right)
$$

where $v$ is the vibration frequency of the molecules in the melt and $\lambda$ is the atomic-scale jump distance of a molecule from the melt to the nucleus (Mondal and Murty, 2006). Changes in $\Delta G^{\prime}{ }_{v}$ imply that as temperature is lowered, the diffusion rate will fall. This will be caused by an increase in the $\eta$ of the melt or solution and will potentially become ratelimiting for any ensuing nucleation (Mullin, 1993).

During crystal growth, incorporation of TAG molecules onto the surface of existing crystals will greatly depend on the driving force or supercooling (Hartel, 2001), with the growth rate in theory increasing with supercooling but being reduced due to the $\eta$ of the melt as the diffusion of a molecule (i.e., mass transfer) is retarded. Importantly, the rate of crystallization of PO has been shown to be inversely proportional to the $\eta$ of the melt (Timms, 1991). A simple model shows a possible approach where $R_{c}$ may be represented as the inverse of $\eta$, as was described previously in Equation 1.8 (Mathlouthi and Genotelle, 1995). At a constant supersaturation, an increase of $\eta$ causes a reduction of $R_{c}$.

The present results show that an alternative to temperature that will increase $\eta$ is the presence of dispersed particles. The oil-sugar blends exhibited a significantly higher $\eta^{*}$ than the bulk oil (Figure 2.10). When considering density differences, $\left(0.9\right.$ and $1.6 \mathrm{~g} \cdot \mathrm{cm}^{-3}$ for oil and sugar, respectively) (Pictet and Vogel, 1928; Simecek-Beatty et al., 2001), the sugar represented $37 \%(\mathrm{v} / \mathrm{v})$ of the oil-sugar blend. On average, the presence of sugar increased $\eta^{*}$ of the bulk control from approximately 6 to $80 \mathrm{~Pa} \cdot \mathrm{s}$. If $\eta^{*}$ may be used as a proxy for $\eta$ as per the Cox-Merz Rule (Cox and Merz, 1958), this near 15-fold increase in $\eta^{*}$ would 


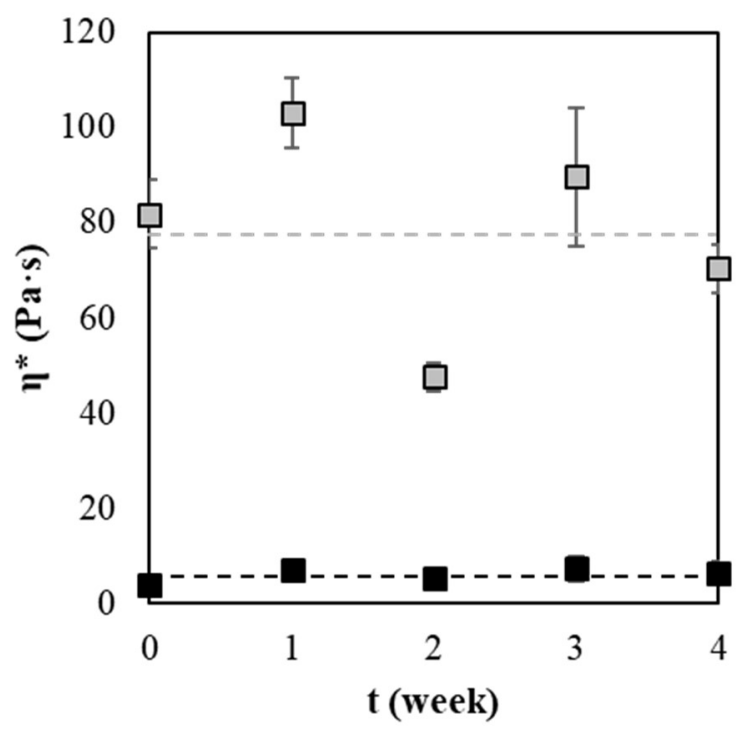

- Sugar $\square+$ Sugar

Figure 2.10 Effect of sugar on $\eta^{*}$ of control over time.

significantly retard nucleation and growth. This effect may be enhanced by loose sugar aggregates that immobilize TAGs leading to a larger effective dispersed phase volume fraction, further increasing $\eta^{*}$ as well as the solid-like character of the fat-sugar matrix, particularly in this stagnant environment, where the diffusion rate will be at a minimum.

Differentiation of $\eta$ and $\eta_{\mu}$ may provide further insight on the role of possible confinement and particle-oil interactions on TAG crystallization. Efforts to characterize oil $\eta_{\mu}$ in the literature are rather limited (Dibildox-Alvarado et al., 2010; Du et al., 2014). Of note, based on work in fat-oil mixtures, Du et al. (2014) stated that changes in $\eta_{\mu}$ were dependent on fractal dimension, but not SFC. Higher fractal dimensions were indicative of oil confinement and thus higher $\eta_{\mu}$. An outcome of both papers is that particle-particle interactions will increase either as temperature is decreased or in the presence of neighbouring particles (e.g., newly-formed fat crystals). In both instances, it will become more difficult for neighbouring oil molecules to move freely due to the increase in $\eta_{\mu}$. Fundamentally, these results clearly 
show that $\eta^{*}$ is an appropriate proxy to predict liquid-state TAG diffusion in particle-oil mixed systems. Such findings suggest that a deeper investigation of the role of $\eta_{\mu}$ in such systems is warranted.

\subsection{Conclusions}

This chapter reports for the first time the role of sugar on SFC, rheology, and fat crystal morphology in PO and its fraction. Notably it was shown that sugar curbed SFC but increased the solid-like character of PO. The presence of sugar also appeared to suppress fat crystal growth. The following chapters will focus specifically on how the addition of sugar affects the way in which PO and PMF respond to processing. Furthermore, on-going studies beyond this project will explore the role of emulsifiers on these properties, which will shed new light on the mechanisms of solidification in particulate-rich fats and oils. 


\section{Chapter 3:3.1}

\section{THE EFFECT OF SUGAR ON RESPONSE TOWARDS COOLING ENDPOINT IN}

\section{PALM OIL}

While the physicochemical properties of bulk oils directly impact their quality and sensory attributes, this relationship is confounded by the presence of added non-fat ingredients such as sugar. Furthermore, the way in which a bulk oil responds to processing may not carry over when mixed with dispersed particles. The purpose of this chapter was to explore the effects of confectioner's sugar and cooling endpoint on SFC, polymorphism, morphology, viscoelasticity, and textural properties of PO and PMF over four weeks of storage. Samples were cooled from $60{ }^{\circ} \mathrm{C}$ to endpoint temperatures of either 15,20 , or $28{ }^{\circ} \mathrm{C}$. Oil-specific models were generated to predict responses based on composition and endpoint. The higher saturated and DAG content of PO enhanced the extent of crystallization, $\mathrm{G}^{\prime}$, and firmness in comparison to PMF. Cooling endpoint temperature also impacted SFC, viscoelasticity, and showed significant interactions with both oil and sugar. This endpoint parameter did not affect firmness, which is closely associated with quality at consumption. The models developed from this study are a first to provide insight and behavioural prediction for the confectionery industry without the need of anecdote.

\footnotetext{
3.1 This chapter previously appeared as a published article with following citation: West, R. and Rousseau, D. (2017). Modelling sugar, processing, and storage effects on palm oil crystallization and rheology. LWT - Food Sci. Technol. 83:201-212.
} 


\subsection{Introduction}

Fat-based confections are composed of non-fat ingredients such as confectioner's sugar, milk solids, or cocoa powder dispersed within a continuous fat phase (Svanberg et al., 2013). The physicochemical properties of these confections determine processing efficiency during unit operations such as extrusion and enrobing, product quality, and organoleptic attributes (Liu et al., 2010).

PO and its fractions are regularly used in confectionery applications given their natural semisolid consistency, low cost, and absence of trans fats (Aftab et al., 2013). Achieving a zero trans fats label is particularly important to industry because of their association with CVD and inflammation (Ascherio et al., 1999). However, PO tends to crystallize slowly because of its high DAG content, which has previously limited its application (Siew and Ng, 1999).

The structure-functionality relationship of fats becomes more complex in the presence of non-fat ingredients where additional interactions and alterations in crystallization pathway occur. In confections, rheology is a product of SFC, crystal morphology, and network development of the fat phase, the composition of the non-fat dispersion, and the resulting particle interactions between both phases (Tang and Marangoni, 2007). Manipulating these

attributes permits significant changes in product properties. For example, with a narrower size distribution and larger volume fraction of sugar, there results a substantial increase in $\eta$ (De Graef et al., 2011) and hardness of the confectionery sample (Afoakwa et al., 2008c).

As the main ingredient in many fat-based confections, sugar imparts both sweetness and mouthfeel while occupying up to $70 \%(\mathrm{v} / \mathrm{v})$ (Jamieson, 2008). The energy barrier for fat crystal nucleation is reduced in its presence as it behaves as a catalytic impurity (Metin and 
Hartel, 2005). The active sites on the surface of sugar particles require fewer liquid-state acylglycerol molecules to assemble and form a nucleus of critical size (Hartel, 2001).

Fat crystallization may be further influenced by processing factors, e.g., cooling rate, shear speed, etc. (Kellens et al., 2007). For example, shear facilitates mass transfer where its speed is positively correlated with crystallization rate and the formation of higher-order polymorphs. Additionally, crystal morphology and alignment may be impacted by shear (De Graef et al., 2006). Cooling temperature is another important processing factor. Whereas $\alpha$ crystals have shown to form readily in PO and later undergo polymorphic transition at temperatures below $20^{\circ} \mathrm{C}, \beta^{\prime}$ crystals form exclusively from the melt at temperatures above $20^{\circ} \mathrm{C}$ (Litwinenko et al., 2002; Foubert et al., 2006). It is well recognized that understanding how processing impacts fat crystallization is necessary to optimize product quality (Afoakwa et al., 2008d).

In this chapter, the effects of confectioner's sugar on the response behaviour of PO and PMF towards cooling endpoint temperature was explored. Samples were characterized over four weeks of storage, which is analogous to the period that many palm-based products undergo prior to grocer distribution. Preliminary oil-specific regression models were generated through multiple regression to predict this behaviour.

\subsection{Materials and Methods}

\subsubsection{Sample Preparation}

Commercial PO and PMF (IOI Loders Croklaan, Channahon, IL, USA) were used in this study. PMF was blended at $65 \%(\mathrm{w} / \mathrm{w})$ with CO (Bunge North America, St. Louis, MS, USA) to obtain similar melting properties as PO according to AOCS Official Method Cd 
16b-93 (Figure 2.1) (Firestone, 1998). TAG and DAG compositions of oils were determined (Table 2.1) as described in Section 2.2.2. Oils were processed either as bulk or combined with sieved 6-x confectioner's sugar (Domino Foods Inc., Yonkers, NY, USA) at $50 \%$ (w/w) using a stand mixer (Hobart Canada Inc., Toronto, ON, Canada). The confectioner's sugar contained $3 \%(\mathrm{w} / \mathrm{w})$ starch, $0.5 \%(\mathrm{w} / \mathrm{w})$ moisture, and possessed an average particle size of $30 \mu \mathrm{m}$.

The samples were heated to $60^{\circ} \mathrm{C}$ for $30 \mathrm{~min}$ at $100 \mathrm{rpm}$ in a scraped-surface jacketed kettle (Tenon Engineering Ltd., Leatherhead, UK) connected to a water bath (VWR International 1157P, Mississauga, ON, Canada) to eliminate crystal memory. Samples were then cooled at $1{ }^{\circ} \mathrm{C} \cdot \mathrm{min}^{-1}$ to a temperature of either 15,20 , or $28^{\circ} \mathrm{C}$. When cooled to either 15 or $28^{\circ} \mathrm{C}$, a thin layer of sample was transferred to a baking sheet and either heated or cooled in an incubator set to either 40 or $0{ }^{\circ} \mathrm{C}$, respectively, until a thermocouple read $20^{\circ} \mathrm{C}$. Because 20 ${ }^{\circ} \mathrm{C}$ is the standard endpoint for processing, this effect of altering final temperature is described as endpoint deviation $(\Delta T)$, according to:

$$
\Delta T=T-T_{e}
$$

where $T$ and $T_{e}$ are the final (i.e., 15,20 , or $28^{\circ} \mathrm{C}$ ) and standard (i.e., $20^{\circ} \mathrm{C}$ ) endpoint temperatures, respectively. In this regard, a final temperature of $15{ }^{\circ} \mathrm{C}$ has an $\Delta T$ of $-5{ }^{\circ} \mathrm{C}$ whereas a final temperature of $28^{\circ} \mathrm{C}$ has an $\Delta T$ of $8{ }^{\circ} \mathrm{C}$. Samples were characterized within 15 min upon reaching $20{ }^{\circ} \mathrm{C}$ (i.e., 0 weeks) or stored at $20{ }^{\circ} \mathrm{C}$ and sampled weekly for 4 weeks. All samples were prepared in triplicate. 


\subsubsection{Pulsed Nuclear Magnetic Resonance}

Fat crystallization was monitored indirectly by measuring SFC via pulsed NMR spectroscopy (Bruker Minispec mq20, Milton, ON, Canada) at $20{ }^{\circ} \mathrm{C}$ using AOCS Official Method Cd 16-81 as described in Section 2.2.3. Samples were injected into glass NMR tubes $(\mathrm{ID}=1 \mathrm{~cm})$ to a height of $4 \mathrm{~cm}$ and stored until analysis. Six sub-replicate measurements were made for each NMR tube.

\subsubsection{Differential Scanning Calorimetry}

DSC (Q2000 and Universal Analysis 2000 software, TA Instruments, New Castle, DE, USA) was used to determine $\Delta \mathrm{H}_{\mathrm{T}}$ of bulk oil and oil-sugar blends. Samples masses $(\sim 10$ mg) were corrected for presence of sugar, if necessary. After equilibrating the sample at 20 ${ }^{\circ} \mathrm{C}$ for $5 \mathrm{~min}$, the temperature was increased to $90{ }^{\circ} \mathrm{C}$ at $5{ }^{\circ} \mathrm{C} \cdot \mathrm{min}^{-1}$, and endotherms corresponding to the $\beta^{\prime}\left(35.4 \pm 1.5^{\circ} \mathrm{C}\right)$ and/or $\beta$ crystals $\left(42.8 \pm 0.8^{\circ} \mathrm{C}\right)$ were identified (Busfield and Proshogo, 1990; Braipson-Danthine and Gibon, 2007). Samples were placed in open aluminum pans for all runs. Nitrogen gas was used to prevent water condensation.

The fraction of $\beta^{\prime}$ polymorph $\left(f_{\beta^{\prime}}\right)$ was measured by:

$$
f_{\beta^{\prime}}=\frac{\Delta H_{\beta^{\prime}}}{\Delta H_{\beta^{\prime}}+\Delta H_{\beta}}
$$

where $\Delta H_{\beta^{\prime}}$ and $\Delta H_{\beta}$ are the enthalpies for $\beta^{\prime}$ and $\beta$ polymorphs, respectively.

\subsubsection{Microscopy}

Confocal laser scanning microscopy (CLSM) (LSM510, Zeiss Inc., Toronto, ON, Canada) was performed to characterize the impact of sugar and processing on fat crystal morphology. 
Fluorol yellow 088 (Sigma-Aldrich, St. Louis, MO, USA) was added to samples at $0.05 \%$ $(\mathrm{w} / \mathrm{w})$ immediately after processing to negatively-stain the fat crystals. Thin layers of sample were placed between a glass slide and cover slip with minimal compression and imaged at $630 \times$ using a $63 \times$ oil immersion objective lens paired with a $10 \times$ ocular lens. The built-in imaging software ZEN 2.3 (Zeiss Inc., Toronto, ON, Canada) was used to assess crystal morphology and size.

\subsubsection{Rheology}

$\mathrm{G}^{\prime}$ and $\mathrm{G}^{\prime \prime}$ were measured using a strain-controlled rheometer (AntonPaar Physica MCR 301, St. Laurent, QC, Canada) and PP25/P2 serrated geometry where frequency sweep tests within the linear viscoelastic region were performed at $0.01 \%$ strain (i.e., 21 points of $\omega$ were collected between 4 and $\left.63 \mathrm{rad} \cdot \mathrm{sec}^{-1}\right) \cdot{ }^{3.2}$ All rheological tests were performed at $20{ }^{\circ} \mathrm{C}$ with a gap size of $1.0 \mathrm{~mm}$.

\subsubsection{Texture Analysis}

Firmness (F) was measured using a texture analyzer (TA.XTplus, Stable Micro Systems, Ltd., Surrey, UK) calibrated with a $30 \mathrm{~kg}$ load cell. Sixty ml plastic cups filled with either bulk oil or oil-sugar blends were stored as above. For analysis, a $45^{\circ}$ steel cone was calibrated to a $10 \mathrm{~mm}$ height above the sample surface and programmed for approach at 2 $\mathrm{mm} \cdot \mathrm{s}^{-1}$. Upon contact with its surface, the cone penetrated the sample at $1 \mathrm{~mm} \cdot \mathrm{s}^{-1}$ for 15 $\mathrm{mm}$ at which point $\mathrm{F}$ was measured.

\footnotetext{
${ }^{3.2}$ Raw frequency sweep tests for $\mathrm{G}^{\prime}$ are provided in $\mathrm{A} 1$.
} 


\subsubsection{Statistical Analysis and Multiple Regression}

A five-way ANOVA from a mixed model using replicate, oil, sugar, endpoint deviation, and storage time (including interactions with storage) was performed to test for significance ( $\mathrm{p}$ $<0.05$ ) with SAS 9.4 (SAS Institute, Cary, NC, USA). ${ }^{3.3}$ Confectioner's sugar was treated as a binary variable where $\zeta=0$ in bulk systems and $\zeta=1$ in oil-sugar blends. Experimentspecific variables (e.g., subreplicate for pulsed NMR, $\omega$ for rheology) were used as needed. Insignificant variables were removed from these models and their degrees of freedom added back into error.

Oil-specific third-order polynomial models ${ }^{3.4}$ with parameters $\Delta T$ and $t$ as independent variables were determined using multiple regression analysis (Snedecor and Cochran, 1989) following the total model:

$$
\hat{\mathrm{y}}=y_{o}+\zeta y_{o s}
$$

where $y_{o}$ and $y_{o s}$ were partial models with $\zeta$-independent and $\zeta$-dependent parameters $\left(x_{i j}\right)$, respectively, as further defined by:

$$
y=\beta_{0}+\sum_{i=1}^{i=2} \beta_{i} x_{i}+\sum_{i=1}^{i=2} \beta_{i} x_{i}^{2}+\sum_{i=1}^{i=2} \beta_{i} x_{i}^{3}+\beta_{i j} x_{i} x_{j}+\beta_{i j} x_{i}^{2} x_{j}+\beta_{i j} x_{i} x_{j}^{2}+\varepsilon
$$

where $j=i+1, \beta$ values are partial regression coefficients and $\varepsilon$ is assumed to be random error from a normal population with zero mean and constant unknown variance. The decision to use third-order polynomials resulted from the lack of explained variance from using second-order polynomials and the little that was gained using fourth-order

\footnotetext{
3.3 ANOVA tables are listed in A2.2.

3.4 Sample calculation of a model generated through multiple regression is provided in A3.1.
} 
polynomials. Coefficients were kept at four decimal places to minimize rounding error. Model limitations such as the inability to extrapolate using processing conditions outside of what was performed experimentally must be acknowledged. Furthermore, $\zeta$ cannot be used as a continuous variable [e.g., $\zeta=0.5$ to predict the effect of $25 \%(\mathrm{w} / \mathrm{w})$ confectioner's sugar on an oil-sugar blend] as this presumes the existence of a linear effect of confectioner's sugar on oil response.

To correct for overfitting, model selection proceeded by Mallows' $C_{P}$ (Mallows, 1973) where the subset model with the lowest $C_{P}$ statistic was selected. This statistic was defined as:

$$
C_{P}=\frac{1}{\sigma^{2}} R S S_{P}-n+2 P
$$

where $R S S_{P}$ is the residual sum of squares, $n$ is the number of observations, and $P$ is the number of parameters (including intercept) within the subset model.

\subsection{Results and Discussion}

\subsubsection{Solid Fat Content and Polymorphism}

The main effects of composition and cooling endpoint on SFC of bulk oil and oil-sugar blends are depicted in Figure 3.1. The main effect of oil on SFC was significant where PO was higher than PMF (e.g., averaged across sugar and endpoint). This was attributed to the higher saturated TAG and DAG content of PO (Table 2.1). Whereas nearly $60 \%$ of PO was composed of palmitic-rich acylglycerols, this number was reduced to $45 \%$ for PMF. Furthermore, the total DAG contents of PO and PMF were $6.4 \%$ and $4.0 \%$, respectively. 

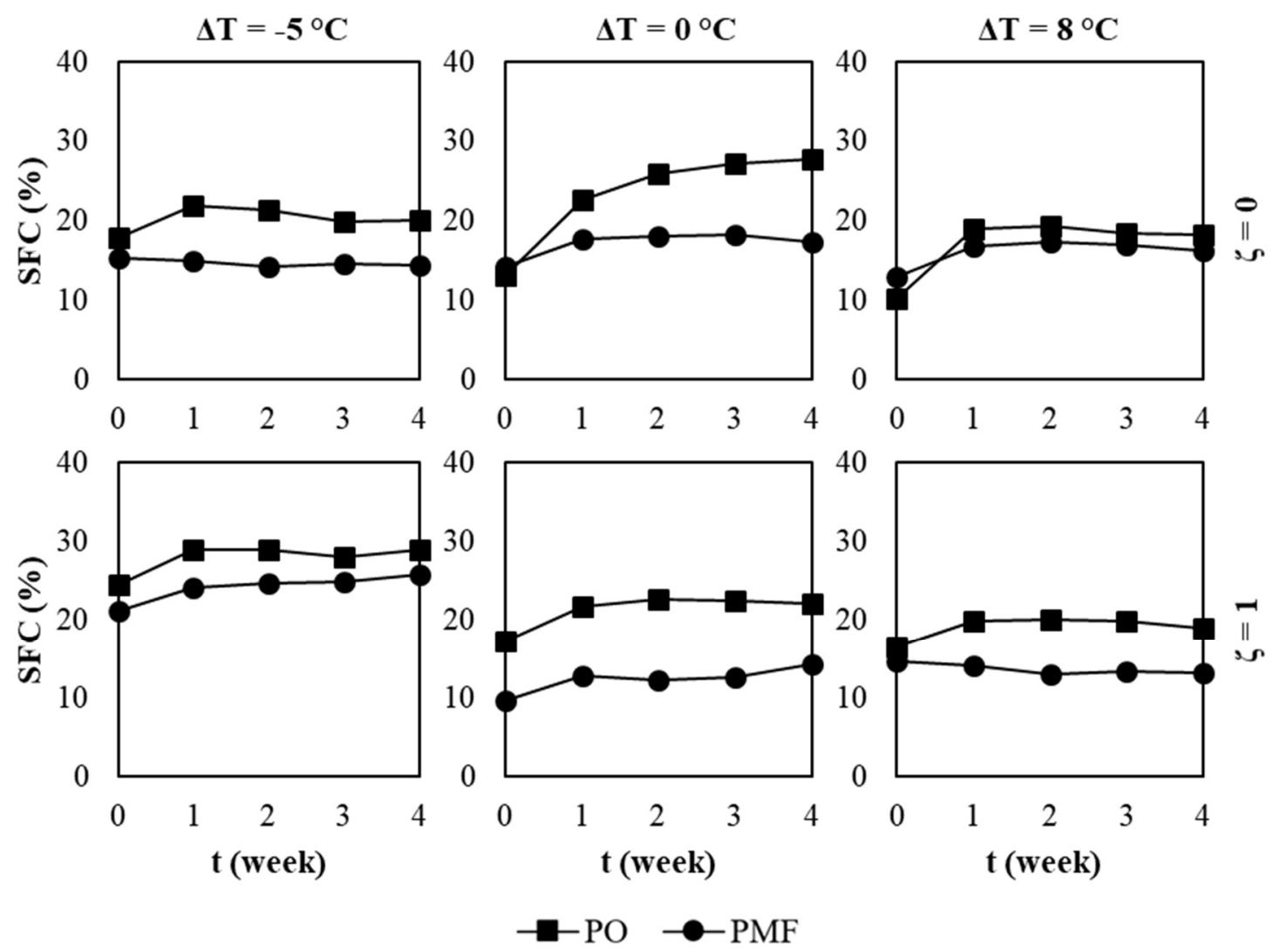

Figure 3.1 Effects of sugar and endpoint on SFC of PO and PMF over time.

A significant interaction existed between sugar and storage where the change in SFC over the first week of storage was greater in bulk oils (Figure 3.1). This may suggest that the addition of confectioner's sugar increases the rate of crystallization, as was noted by the earlier plateau in SFC in the oil-sugar blends (Toro-Vazquez et al., 2002). The presence of foreign surfaces requires fewer liquid-state TAGs to assemble a nucleus of critical size thus allowing nucleation to proceed more readily (Hartel, 2001). In contrast, fewer nucleation sites in the bulk oils resulted in a greater change of SFC upon the first week of storage.

As for polymorphism, essentially $\beta^{\prime}$ crystals were observed to form in the presence of confectioner's sugar with only a small portion of $\beta$ crystals (1-3 \%) forming in PO-sugar blends (Table 3.1). Although sugar induces fat nucleation, it was previously reported that 
Table 3.1 Effects of sugar and endpoint on $f_{6^{\prime}}$ for PO and PMF over time. ${ }^{3.5}$

\begin{tabular}{|c|c|c|c|c|c|c|c|}
\hline \multirow{2}{*}{$\zeta$} & \multirow{2}{*}{$t$ (week) } & \multicolumn{3}{|c|}{ PO } & \multicolumn{3}{|c|}{ PMF } \\
\hline & & $\Delta T=-5^{\circ} \mathrm{C}$ & $\Delta T=0^{\circ} \mathrm{C}$ & $\Delta T=8^{\circ} \mathrm{C}$ & $\Delta T=-5^{\circ} \mathrm{C}$ & $\Delta T=0^{\circ} \mathrm{C}$ & $\Delta T=8^{\circ} \mathrm{C}$ \\
\hline \multirow{5}{*}{0} & 0 & $0.87 \pm 0.03$ & $0.74 \pm 0.06$ & $0.80 \pm 0.06$ & $1.00 \pm 0.00$ & $0.90 \pm 0.08$ & $0.77 \pm 0.14$ \\
\hline & 1 & $0.87 \pm 0.12$ & $0.79 \pm 0.03$ & $0.75 \pm 0.03$ & $1.00 \pm 0.00$ & $0.91 \pm 0.08$ & $0.73 \pm 0.11$ \\
\hline & 2 & $0.74 \pm 0.02$ & $0.83 \pm 0.03$ & $0.73 \pm 0.02$ & $1.00 \pm 0.00$ & $0.91 \pm 0.03$ & $0.78 \pm 0.04$ \\
\hline & 3 & $0.85 \pm 0.06$ & $0.86 \pm 0.02$ & $0.76 \pm 0.04$ & $1.00 \pm 0.00$ & $0.93 \pm 0.07$ & $0.67 \pm 0.18$ \\
\hline & 4 & $0.84 \pm 0.10$ & $0.88 \pm 0.01$ & $0.77 \pm 0.05$ & $1.00 \pm 0.00$ & $0.87 \pm 0.02$ & $0.76 \pm 0.07$ \\
\hline \multirow{5}{*}{1} & 0 & $1.00 \pm 0.00$ & $1.00 \pm 0.00$ & $0.98 \pm 0.04$ & $1.00 \pm 0.00$ & $1.00 \pm 0.00$ & $1.00 \pm 0.00$ \\
\hline & 1 & $0.99 \pm 0.01$ & $1.00 \pm 0.00$ & $0.98 \pm 0.03$ & $1.00 \pm 0.00$ & $1.00 \pm 0.00$ & $1.00 \pm 0.00$ \\
\hline & 2 & $0.99 \pm 0.01$ & $1.00 \pm 0.00$ & $0.98 \pm 0.04$ & $1.00 \pm 0.00$ & $1.00 \pm 0.00$ & $1.00 \pm 0.00$ \\
\hline & 3 & $0.99 \pm 0.01$ & $1.00 \pm 0.00$ & $0.97 \pm 0.05$ & $1.00 \pm 0.00$ & $1.00 \pm 0.00$ & $1.00 \pm 0.00$ \\
\hline & 4 & $1.00 \pm 0.01$ & $1.00 \pm 0.00$ & $0.97 \pm 0.05$ & $1.00 \pm 0.00$ & $1.00 \pm 0.00$ & $1.00 \pm 0.00$ \\
\hline
\end{tabular}

\footnotetext{
${ }^{3.5}$ Means with standard deviations.
} 
metastable polymorphs were formed as a result (Dhonsi and Stapley, 2006). In either bulk oil, $\beta$ crystals formed more readily, with the $f_{\beta^{\prime}}$ of bulk PO generally lower than in bulk PMF. PMF is a compositionally simpler oil than PO because of its fractionation and it has been previously established that acylglycerol composition can impact polymorphism where $\beta$ crystal growth is promoted as compositional complexity is reduced (Rousseau, 2007). Interestingly, there was no significant trend in $f_{\beta^{\prime}}$ with respect to storage time, indicating that polymorphic composition was generally maintained during crystallization.

There existed a significant interaction between cooling endpoint and storage where the change in SFC over time was smallest when the endpoint was $5{ }^{\circ} \mathrm{C}$ (Figure 3.1) (e.g., averaged across composition). Since the process of fat crystallization from the melt require the reduction of activation free energy (Hartel, 2001), this suggested that the system had further shifted from equilibrium which increased the rate of nucleation as the endpoint temperature decreased.

While final SFC (e.g., week 4) was greatest when bulk oils were cooled to $20^{\circ} \mathrm{C}$, particularly with PO, this was not the case in the presence of confectioner's sugar. Instead, final SFC was greatest when the oil-sugar blends were cooled to $15{ }^{\circ} \mathrm{C}$. This deviation in processing behaviour that is created by confectioner's sugar highlights the inability to use trends and anecdote generated from bulk oils towards more complex systems. Through multiple regression analysis, subset models of $\mathrm{PO}$ and PMF to predict SFC were generated which may circumvent these limitations. These subset models were:

$$
\begin{aligned}
\hat{\mathrm{y}}=13.6824+ & 12.6795 t+0.0712 \Delta T^{2}-4.5396 t^{2}-0.0154 \Delta T^{3} \\
& +0.5766 t^{3}+0.5007 \Delta T t-0.0660 \Delta T^{2} t-0.0512 \Delta T t^{2} \\
& +\zeta\left(3.7736-1.3186 \Delta T-6.2447 t+1.9367 t^{2}+0.0250 \Delta T^{3}\right. \\
& \left.-0.2521 t^{3}-0.4863 \Delta T t+0.0572 \Delta T^{2} t+0.0486 \Delta T t^{2}\right)
\end{aligned}
$$


$\left(C_{P}=18.00, \mathrm{~F}_{17,522}=354.48, \mathrm{p}<0.0001\right)$ and:

$$
\begin{aligned}
\hat{\mathrm{y}}=14.9922+ & 0.2312 \Delta T+3.4077 t-1.2800 t^{2}-0.0079 \Delta T^{3}+0.1525 t^{3} \\
& +0.3393 \Delta T t-0.0121 \Delta T^{2} t-0.0584 \Delta T t^{2} \\
& +\zeta\left(-5.0967-4.3329 \Delta T-0.2042 t^{2}+0.0808 \Delta T^{3}\right. \\
& \left.+0.0727 t^{3}-0.5441 \Delta T t+0.0923 \Delta T t^{2}\right)
\end{aligned}
$$

$\left(C_{P}=14.55, \mathrm{~F}_{15,524}=351.83, \mathrm{p}<0.0001\right)$ for $\mathrm{PO}$ and PMF, respectively.

PO appeared to be suppressed in SFC when cooled to $28^{\circ} \mathrm{C}$, regardless of the presence of sugar (Figure 3.1). In contrast, the response of PMF towards cooling endpoint seemed to be more sensitive in the presence of confectioner's sugar. Whereas a suppression of SFC was observed when bulk PMF was cooled to $15{ }^{\circ} \mathrm{C}$, this was not so with PMF-sugar blends. Instead, a suppression was observed if this blend was cooled to $28^{\circ} \mathrm{C}$.

Increasing the endpoint temperature appeared to decrease $f_{\beta^{\prime}}$ and promote $\beta$ crystal growth in the bulk oils (Table 3.1). This was particularly prominent with PMF where $\beta$ crystals comprised 0,10 , and $26 \%$ of the system as the endpoint increased from 15 to 20 to $28{ }^{\circ} \mathrm{C}$, respectively. This correlation between endpoint and polymorphism was consistent with literature (Litwinenko et al., 2002; Foubert et al., 2006).

\subsubsection{Fat Crystal Morphology}

The impact of confectioner's sugar and cooling endpoint on fat crystal morphology of bulk PO and PMF at 0 weeks is depicted in Figures 3.2 and 3.3, respectively. While polymorphism may be assigned according to morphology under static crystallization (Hartel, 2001), this no longer holds true once shear has been implemented. In the absence of confectioner's sugar, both oils exhibited spheroids varying in definition and density. For 

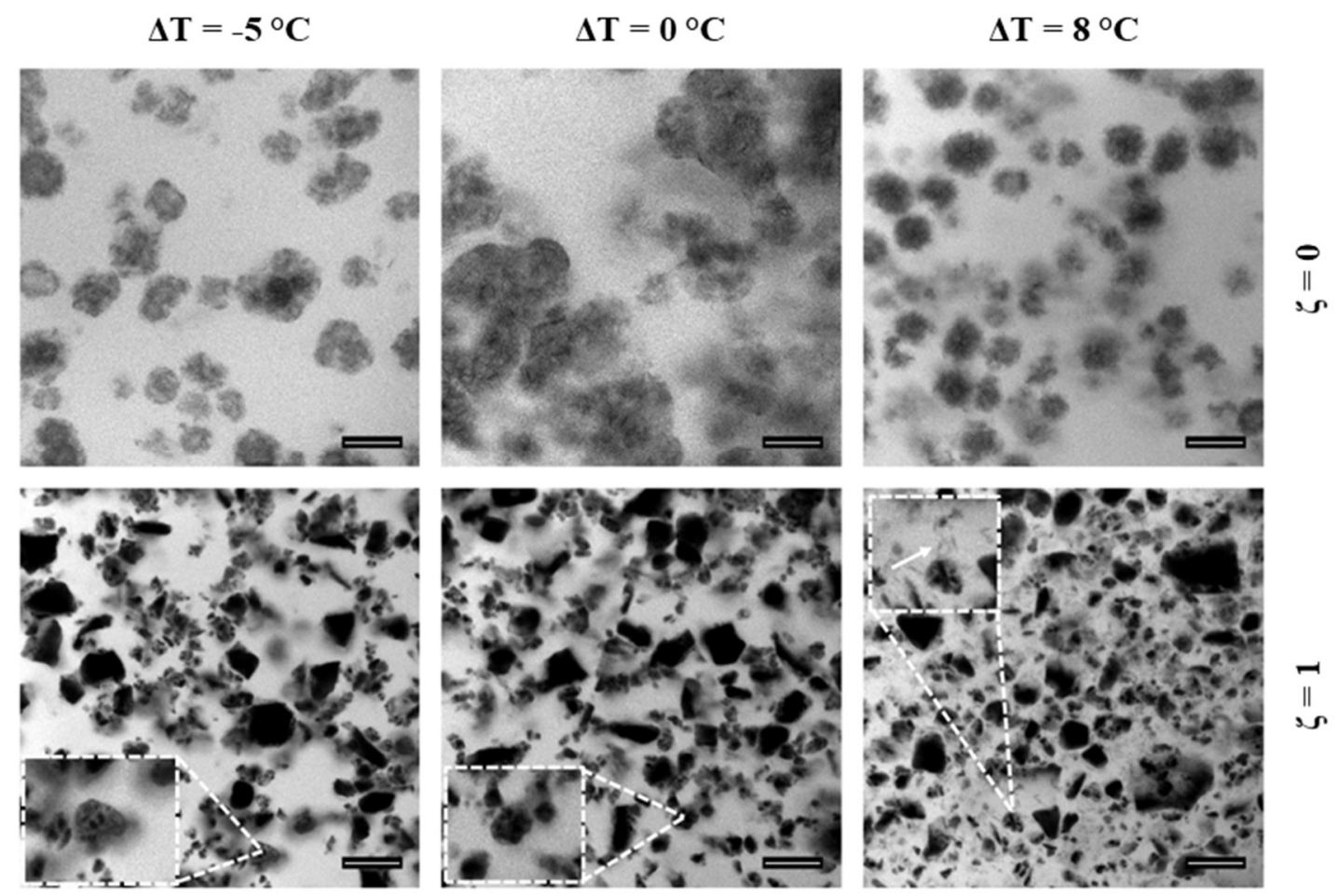

Figure 3.2 Effects of sugar and endpoint on PO crystal morphology at week 0 (bar $=20$ $\mu m)$.

$\Delta \mathrm{T}=-5^{\circ} \mathrm{C}$
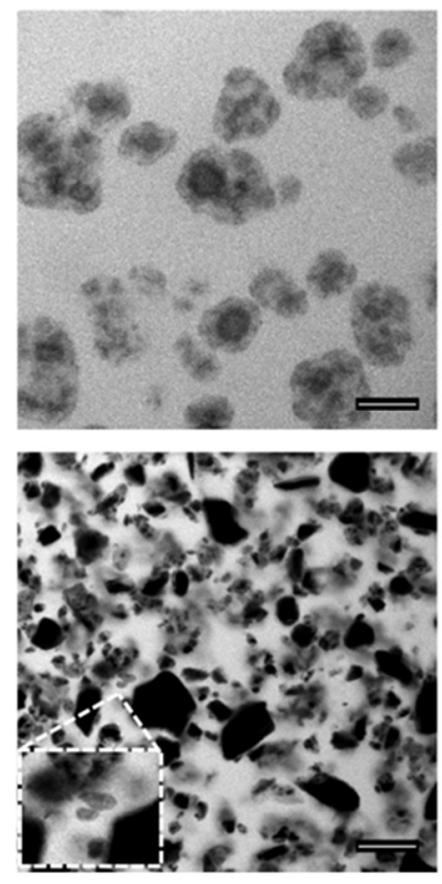

$\Delta \mathbf{T}=\mathbf{0}^{\circ} \mathbf{C}$
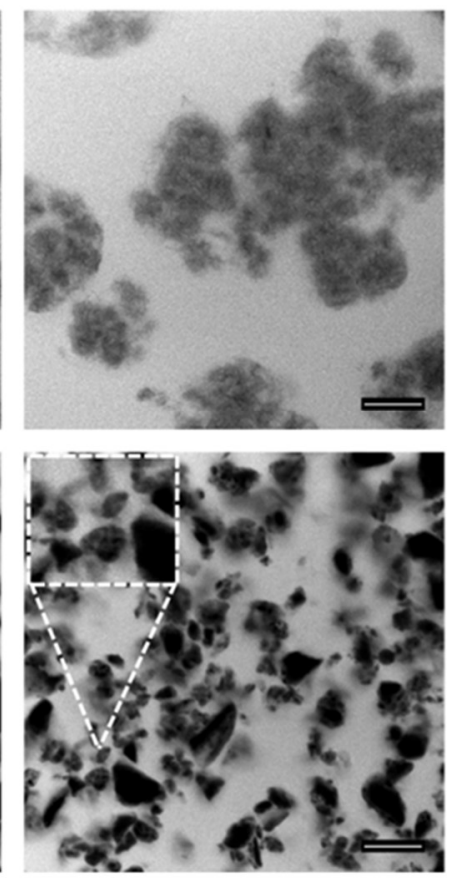

$\Delta \mathrm{T}=\mathbf{8}^{\circ} \mathrm{C}$
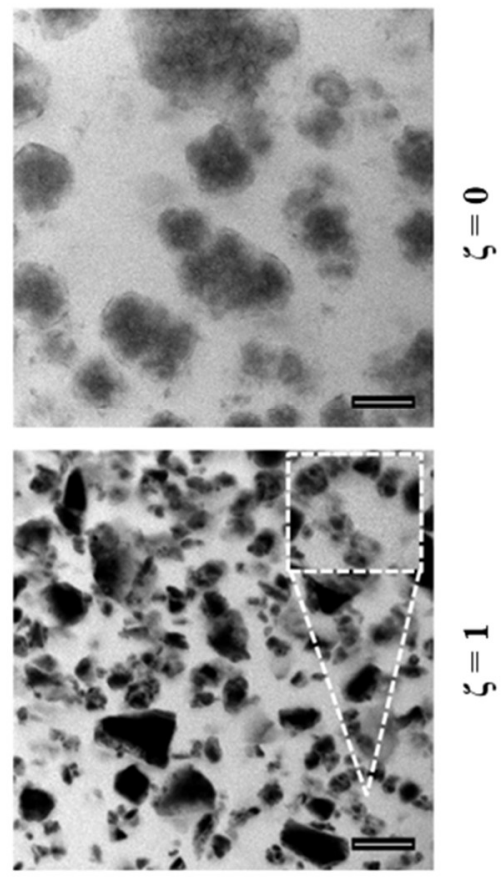

Figure 3.3 Effects of sugar and endpoint on PMF crystal morphology at week 0 (bar $=20$ $\mu m)$. 
bulk PO, spheroids were $\leq 22 \mu \mathrm{m}$ in diameter and showed slight clustering when the endpoint was $15{ }^{\circ} \mathrm{C}$. Bulk PMF showed crystals similar in morphology and were slightly larger in diameter, i.e., $\leq 30 \mu \mathrm{m}$, under the same endpoint. Spheroidal crystals grew in diameter, i.e., $\leq 45 \mu \mathrm{m}$, and the degree of clustering increased in both oils when cooled to $20{ }^{\circ} \mathrm{C}$. When the endpoint was $28{ }^{\circ} \mathrm{C}$, crystals reduced in diameter, i.e., $\leq 18 \mu \mathrm{m}$, and did not cluster in bulk PO. Little difference was observed when bulk PMF was cooled to either 20 or $28^{\circ} \mathrm{C}$.

The impact of confectioner's sugar and cooling endpoint on the crystal morphology of bulk oils at week 4 is depicted in Figures 3.4 and 3.5, respectively. Across all endpoint temperatures, crystals were slightly smaller in diameter and appeared denser in both oils apart from bulk PMF cooled to $20^{\circ} \mathrm{C}$. Additionally, the formation of a network of acicular crystals (highlighted with arrows) was observed as early as one week in either oil. The growth of these needle-like crystals under static conditions is typical of $\beta^{\prime}$ and $\beta$ crystal morphologies (Hartel, 2001). This network appeared to be further developed in bulk PO compared to bulk PMF, which is a result of its higher saturated TAG content (Table 2.1) and SFC (Figure 3.1). For bulk PMF, the network generally appeared to be composed of finer, shorter secondary crystals. Interestingly, an inverse relationship between crystal size and network development was observed in either bulk oil (Figures 3.4 and 3.5).

The crystal morphology of either oil was significantly altered by the addition of confectioner's sugar. In Chapter 2, the ability to characterize fat crystals in the presence of confectioner's sugar using conventional brightfield microscopy had failed — likely due to sample density. Here, CLSM appeared to have resolved this issue. Prior to storage, smooth, 
$\Delta \mathrm{T}=-5^{\circ} \mathrm{C}$
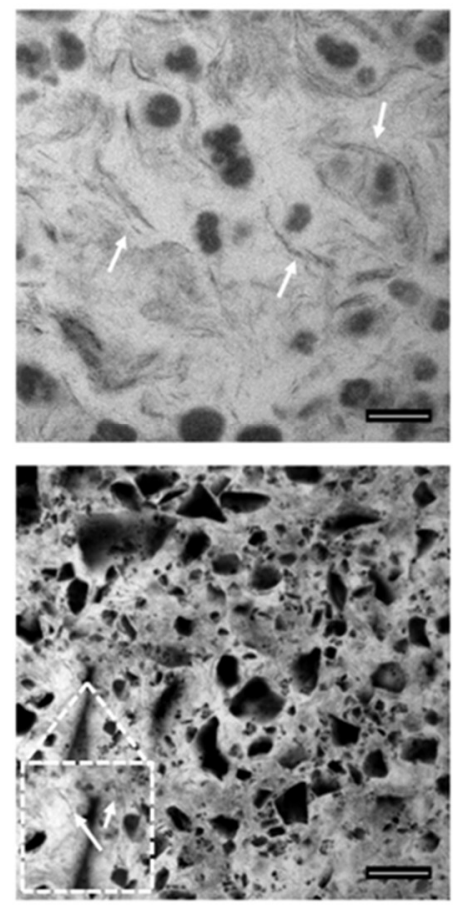

$\Delta \mathrm{T}=0^{\circ} \mathrm{C}$
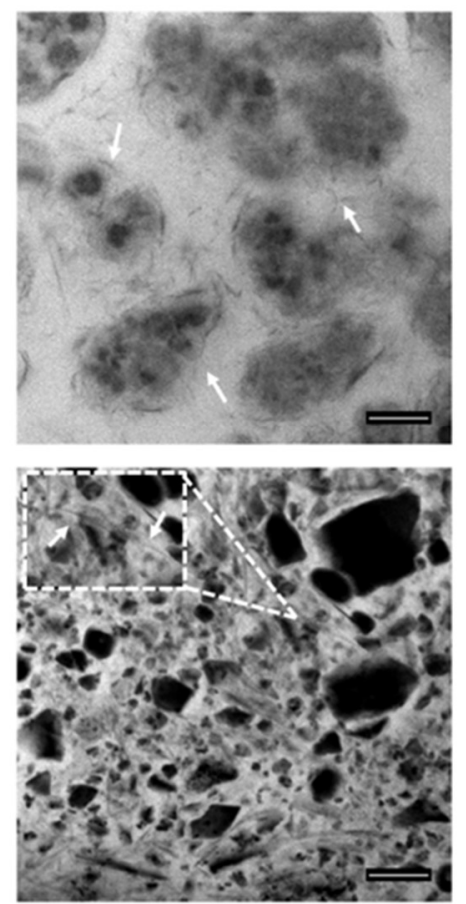

$\Delta \mathbf{T}=\mathbf{8}^{\circ} \mathrm{C}$
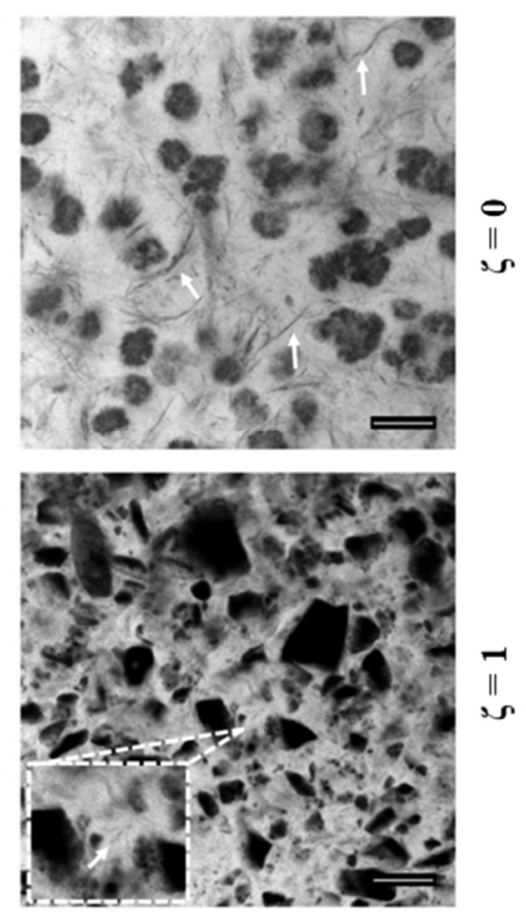

Figure 3.4 Effects of sugar and endpoint on PO crystal morphology at week 4 (bar $=20$ $\mu m)$.
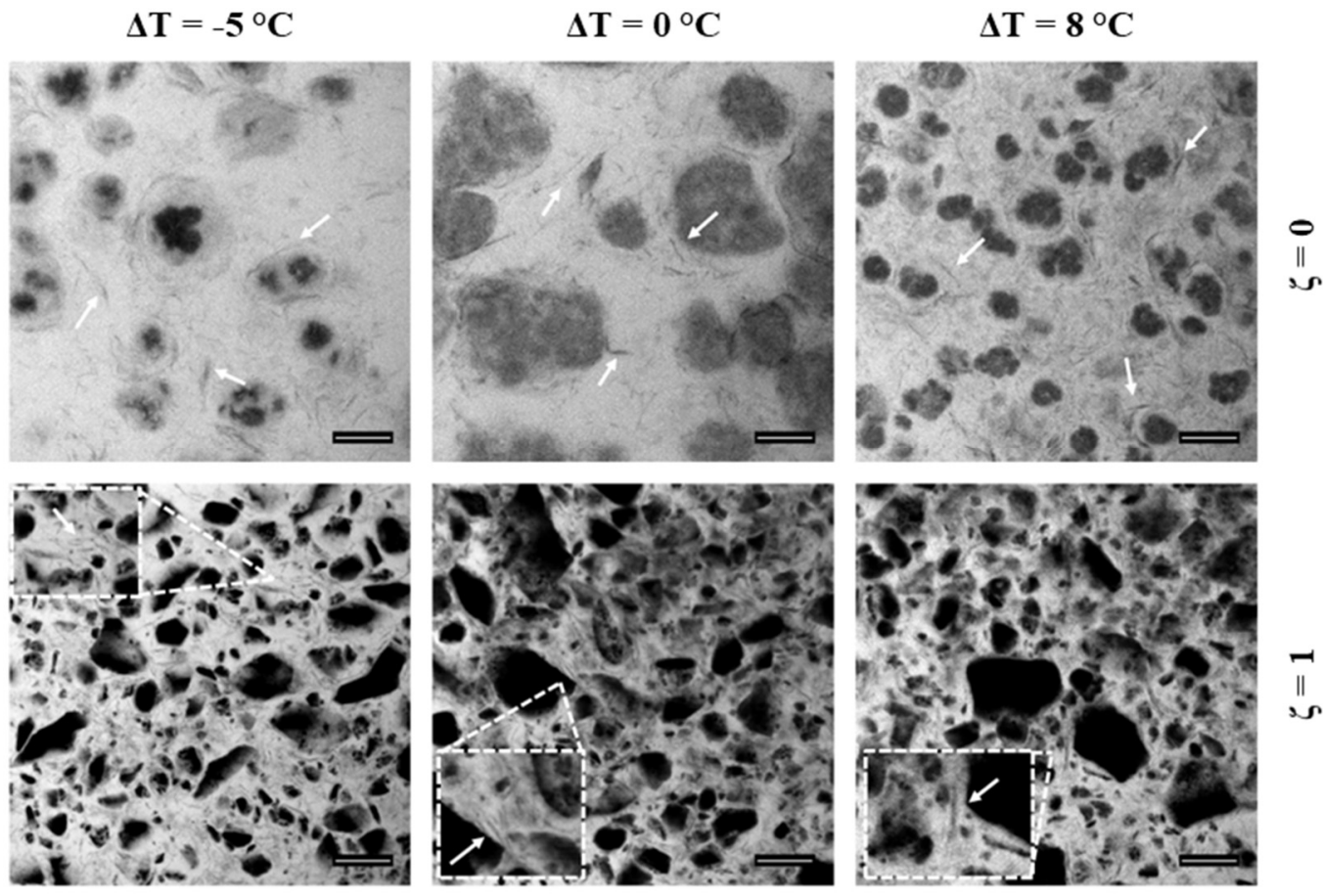

Figure 3.5 Effects of sugar and endpoint on PMF crystal morphology at week 4 (bar $=20$ $\mu m)$. 
obloid fat crystals were observed in both oils (insets, Figures 3.2 and 3.3). These crystals were much smaller in diameter, i.e., $\leq 11 \mu \mathrm{m}$, compared to their bulk counterparts and appeared to have inclusions of fine particles from confectioner's sugar. Svanberg et al. (2011a) previously reported that CB crystals grew on the surface of sugar particles in nonseeded blends, however, the primary crystals in these oil-blends did not appear to be localized at particle surfaces. Further noted is what appeared to be additional fine secondary crystals at week 0 in PO when the endpoint was $28^{\circ} \mathrm{C}$ (arrow, inset, Figure 3.2). Secondary acicular crystals were observed in both oil-sugar blends upon storage (highlighted with arrows, Figures 3.4 and 3.5). For PO, the secondary crystals appeared longest when the endpoint was $20^{\circ} \mathrm{C}$, i.e., $\leq 40 \mu \mathrm{m}$. When cooled to either 15 or $28^{\circ} \mathrm{C}$, these crystals appeared much finer and shorter. The impact of cooling endpoint on network development was less significant in PMF-sugar blends.

\subsubsection{Viscoelasticity}

The rheology of food is of industrial importance because of its close relationship to processing efficiency and sensory properties (Liu et al., 2010). The main effects of composition and cooling endpoint on $\mathrm{G}^{\prime}$ of bulk oil and oil-sugar blends are depicted in Figure 3.6. Although both oils increased in $\mathrm{G}^{\prime}$ upon storage, $\mathrm{PO}$ generally exhibited greater $\mathrm{G}^{\prime}$ compared to PMF. While SFC and morphology are thought to directly impact viscoelasticity (Tang and Marangoni, 2007), this greater $\mathrm{G}^{\prime}$ in $\mathrm{PO}$ was a product of its higher saturated TAG and DAG content (Table 2.1) and further developed crystal network (Figure 3.4) in comparison to PMF (Figure 3.5). Similar trends were observed with G" (Figure 3.7). 

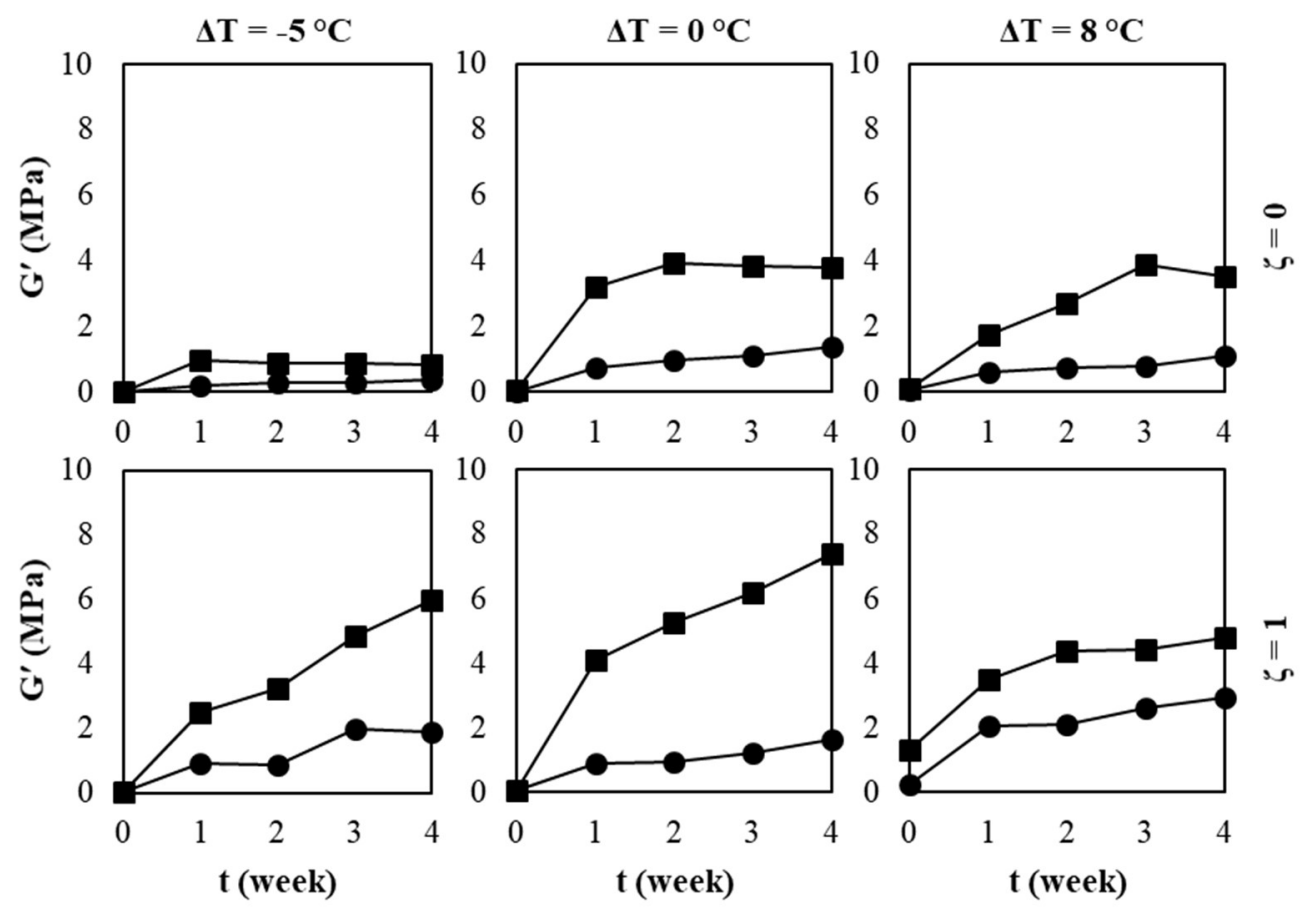

$\rightarrow-\mathrm{PO} \rightarrow \mathrm{PMF}$

Figure 3.6 Effects of sugar and endpoint on $G^{\prime}$ of $P O$ and $P M F$ over time.
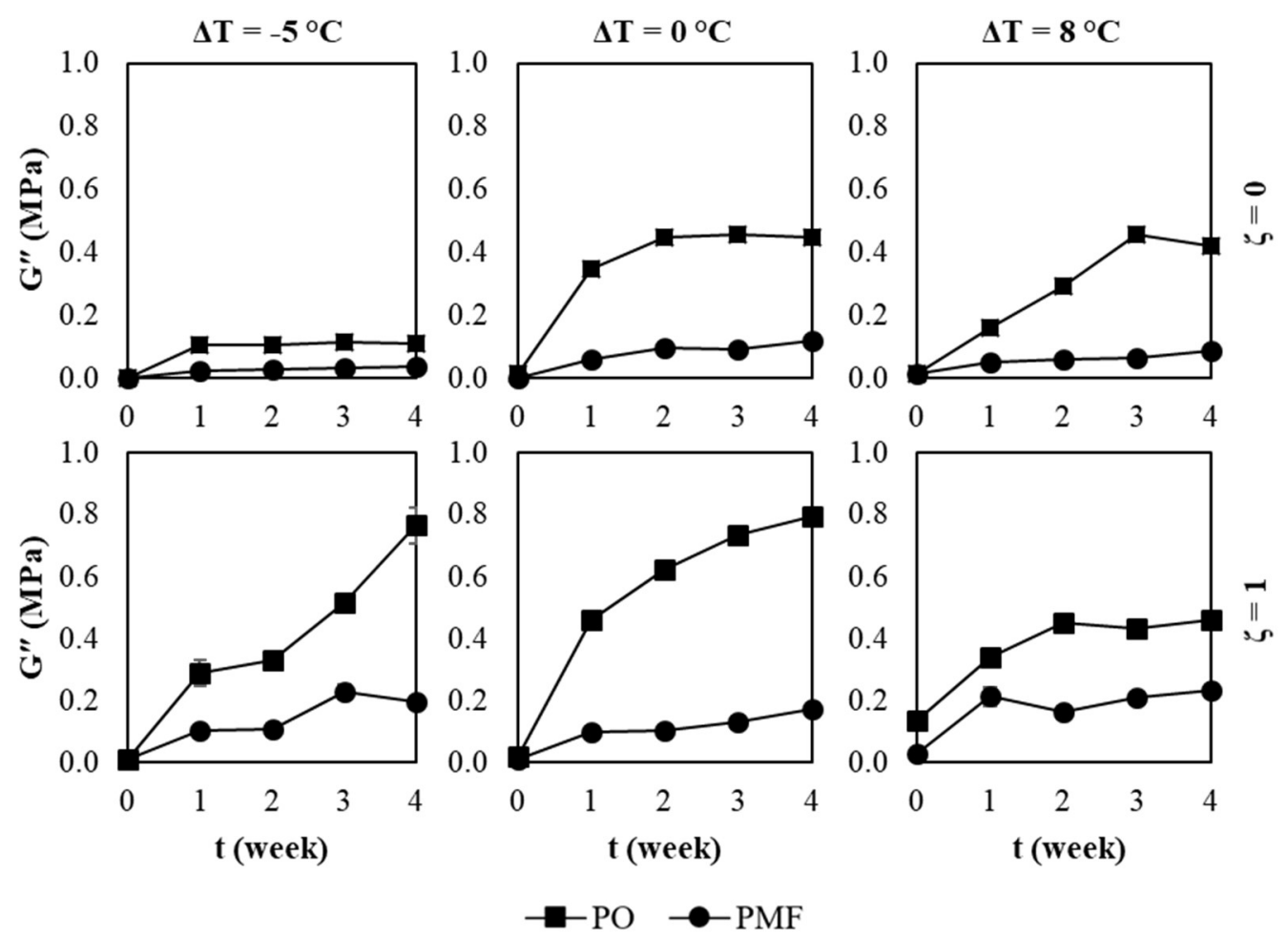

Figure 3.7 Effects of sugar and endpoint on $G^{\prime \prime}$ of PO and PMF over time. 
The main effect of confectioner's sugar was significant for both $\mathrm{G}^{\prime}$ and $\mathrm{G}^{\prime \prime}$ where the viscoelastic properties of the crystal network were enhanced by its addition. Although larger spheroids and longer secondary crystals were observed in bulk systems, the way in which the finer, shorter network crystals grew to encapsulate particles in the oil-sugar blends (Figures 3.4 and 3.5) resulted in tighter, more rigid networks that led to significantly higher $\mathrm{G}^{\prime}$ (Figure 3.6) and G" (Figure 3.7) values. This raised the question about the specific nature of confectioner's sugar, e.g., active filler, in such systems.

Significant interactions between sugar and storage time were observed for $\mathrm{G}^{\prime}$ and $\mathrm{G}^{\prime \prime}$ where the rate of change in viscoelastic properties during storage was greater in the presence of confectioner's sugar. This was especially true for $\mathrm{G}^{\prime}$ and $\mathrm{G}^{\prime \prime}$ in blends made specifically with PO (Figures 3.6 and 3.7). Whereas these oil-sugar blends were suggested to be closer to equilibrium based on SFC (Figure 3.1), that was based solely on the solid fraction of the oil. The continued evolution of these blends according to their viscoelastic properties (Figures 3.6 and 3.7) suggested possible rearrangement of the crystal network around these particles and minimization of free energy where the solid-state fraction remained constant (Metin and Hartel, 2005). Furthermore, the presence of confectioner's sugar was previously shown to increase oil viscosity in liquid oil (Figure 2.10) and may belabour diffusivity of liquid-state acylglycerols towards the surface of growing fat crystals. Additional considerations to explore the impact of confectioner's sugar on PO crystallization may include characterization of oil $\eta_{\mu}$ and tortuosity (Maleky and Marangoni, 2011; Du et al., 2014).

With viscoelasticity of PO and PMF being significantly impacted by the addition of confectioner's sugar and exhibiting trends over storage different from their bulk 
counterparts, subset models were created to take these interactions into consideration. Subset models of $\mathrm{G}^{\prime}$ for $\mathrm{PO}$ and $\mathrm{PMF}$ were determined to be:

$$
\begin{aligned}
& \hat{\mathrm{y}}=0.6658+ 0.3576 \Delta T+2.6175 t-0.7245 t^{2}-0.0072 \Delta T^{3}+0.0709 t^{3} \\
&+0.1073 \Delta T t-0.0093 \Delta T^{2} t-0.0056 \Delta T t^{2} \\
&+\zeta\left(-0.1582+0.0455 \Delta T+1.5576 t+0.0178 \Delta T^{2}\right. \\
&-0.5332 t^{2}+0.0930 t^{3}-0.0699 \Delta T t-0.0030 \Delta T^{2} t \\
&\left.-0.0068 \Delta T t^{2}\right) \\
&\left(C_{P}=18.00, \mathrm{~F}_{17,1866}=902.68, \mathrm{p}<0.0001\right) \text { and }: \\
& \hat{\mathrm{y}}=0.0374+ 0.0152 \Delta T+0.8860 t-0.0013 \Delta T^{2}-0.3152 t^{2}+0.0452 t^{3} \\
&+0.0243 \Delta T t-0.0046 \Delta T^{2} t \\
&+\zeta\left(0.3172 t+0.0051 \Delta T^{2}-0.1361 t^{2}+0.0161 t^{3}\right. \\
&\left.+0.0132 \Delta T t+0.0090 \Delta T^{2} t-0.0104 \Delta T t^{2}\right)
\end{aligned}
$$

$\left(C_{P}=13.70, \mathrm{~F}_{14,1866}=573.54, \mathrm{p}<0.0001\right)$, respectively. For $\mathrm{G}^{\prime \prime}$, subset models were:

$$
\begin{aligned}
\hat{\mathrm{y}}=0.0857+ & 0.2184 t-0.0028 \Delta T^{2}-0.0302 t^{2}+0.0002 \Delta T^{3}+0.0103 \Delta T t \\
& -0.0011 \Delta T^{2} t \\
& +\zeta\left(0.2392 t-0.1134 t^{2}+0.0002 \Delta T^{3}+0.0194 t^{3}\right. \\
& \left.-0.0052 \Delta T t-0.0024 \Delta T t^{2}\right)
\end{aligned}
$$

$\left(C_{P}=11.73, \mathrm{~F}_{8,1874}=430.17, \mathrm{p}<0.0001\right)$ for $\mathrm{PO}$ and:

$$
\begin{aligned}
\hat{\mathrm{y}}=0.0039+ & 0.0011 \Delta T+0.0741 t-0.0251 t^{2}+0.0035 t^{3}+0.0019 \Delta T t \\
& -0.0004 \Delta T^{2} t \\
& +\zeta\left(0.0565 t+0.0006 \Delta T^{2}-0.0245 t^{2}+0.0032 t^{3}\right. \\
& \left.+0.0007 \Delta T^{2} t-0.0008 \Delta T t^{2}\right)
\end{aligned}
$$

$\left(C_{P}=9.66, \mathrm{~F}_{8,1872}=167.28, \mathrm{p}<0.0001\right)$ for PMF, respectively.

With the consideration of cooling endpoint, significant interactions also existed for $\mathrm{G}^{\prime}$ and $\mathrm{G}^{\prime \prime}$. Viscoelasticity was greatest for either bulk oil when the endpoint was $20{ }^{\circ} \mathrm{C}$ which correlated with SFC (Figure 3.1), spheroid diameter, and degree of clustering (Figures 3.23.5). In contrast, a suppression was observed in the bulk oils if cooled to $15^{\circ} \mathrm{C}$ (Figures 3.6 
and 3.7). Furthermore, the oil-sugar blends responded differently to cooling endpoint upon storage. For example, final $\mathrm{G}^{\prime}$ and $\mathrm{G}^{\prime \prime}$ (e.g., week 4) of PO-sugar blends were greatest when cooled to $20^{\circ} \mathrm{C}$ (Figures 3.6 and 3.7), which was consistent with network crystal lengths (Figure 3.4). In contrast, final $\mathrm{G}^{\prime}$ and $\mathrm{G}^{\prime \prime}$ were most suppressed when the sample was cooled to $28{ }^{\circ} \mathrm{C}$. For PMF-sugar blends, an endpoint temperature of $28{ }^{\circ} \mathrm{C}$ promoted final $\mathrm{G}^{\prime}$ whereas no differences were observed when cooled to either 15 or $20^{\circ} \mathrm{C}$. The impact that confectioner's sugar has on the response of viscoelasticity towards cooling endpoint were also accounted for in subset models 3.7 and 3.8.

\subsubsection{Firmness}

Large deformation experiments are used to determine the textural properties of foods, such as F, and are regularly used in conjunction with oscillatory rheology (Omar et al., 2005). The main effects of composition and cooling endpoint on F of bulk oil and oil-sugar blends are depicted in Figure 3.8. Regardless of sugar or endpoint, PO was generally firmer than PMF, which was a result of its higher saturated TAG and DAG (Table 2.1). This was also corroborated both with its further developed network (Figures 3.4) and greater viscoelastic properties (Figures 3.6 and 3.7). There also existed a significant interaction between oil and sugar where the increase in F created by sugar was much greater for PO than PMF (Figure $3.8)$.

A deep understanding of how fat crystallization is affected by processing is critical towards achieving proper texture and quality (Debaste et al., 2008). While there existed a significant main effect of cooling endpoint on $\mathrm{F}$, where a slight suppression of $\mathrm{F}$ was observed when 

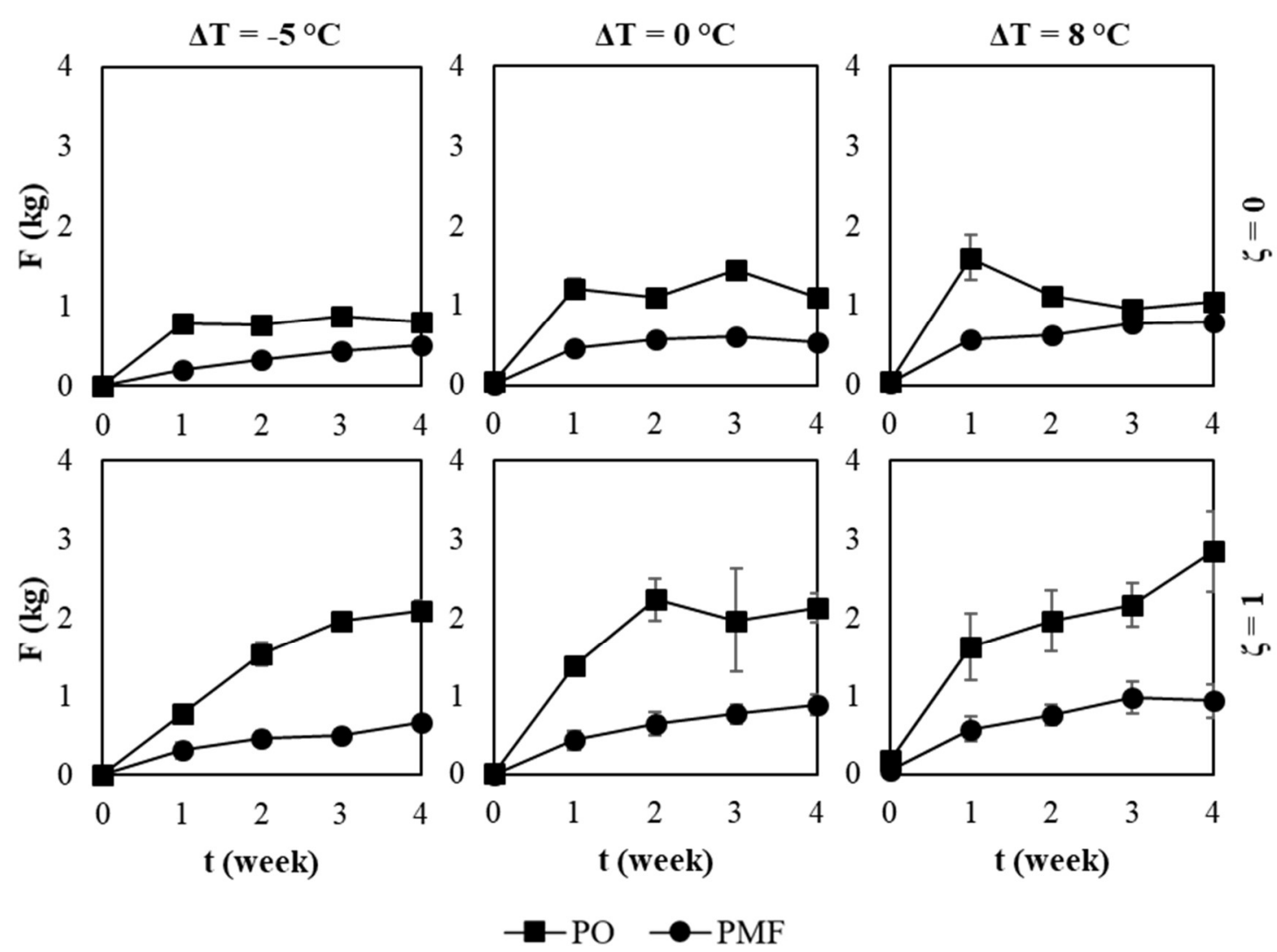

Figure 3.8 Effects of sugar and endpoint on $\mathrm{F}$ of $\mathrm{PO}$ and PMF over time.

cooled to $15^{\circ} \mathrm{C}$, no significant interaction between endpoint and any of the main effects was observed. Perhaps most importantly was that no significant difference in final F (e.g., week 4) was observed when the oil-sugar blends were cooled to different endpoint temperatures (Figure 3.8) despite initial F (e.g., week 0) having increased by as much as $3700 \%$. Industrially, this may be of benefit as $\mathrm{F}$ is an important sensory property. Production efficiency, e.g., pumping, extrusion, etc., may be achieved through modifying cooling temperatures, and thus initial $\mathrm{F}$, with assurance that final $\mathrm{F}$ remains unchanged.

Multiple regression analysis determined the subset models for $\mathrm{F}$ to be:

$$
\begin{aligned}
\hat{\mathrm{y}}=0.1813+ & 0.1150 \Delta T+1.5262 t-0.6431 t^{2}-0.0018 \Delta T^{3}+0.0801 t^{3} \\
& +\zeta\left(0.2871 t+0.0018 \Delta T^{2} t\right)
\end{aligned}
$$


$\left(C_{P}=0.36, \mathrm{~F}_{8,81}=26.75, \mathrm{p}<0.0001\right)$ for $\mathrm{PO}$ and:

$$
\begin{aligned}
\hat{\mathrm{y}}=0.0206+ & 0.4894 t-0.1563 t^{2}+0.0177 t^{3}+0.0221 \Delta T t-0.0042 \Delta T t^{2} \\
& +\zeta(0.0497 t)
\end{aligned}
$$

$\left(C_{P}=0.14, \mathrm{~F}_{6,83}=62.61, \mathrm{p}<0.0001\right)$ for PMF, respectively.

\subsection{Conclusions}

The effect of confectioner's sugar on the response towards cooling temperatures were established in this chapter. Furthermore, preliminary subset models were generated through multiple regression to predict such behaviour. While the addition of confectioner's sugar enhanced crystallization and suppressed $\beta$ crystal formation, these oil-sugar blends continued to evolve in viscoelasticity and texture over time due to continued recrystallization. This was in stark contrast to their bulk counterparts. Of interest was the ability to modify initial $\mathrm{F}$ through cooling endpoint without impacting final $\mathrm{F}$ as this may be used to regulate production efficiency. With this preliminary insight towards the impact that confectioner's sugar has on processing behaviour, the next chapter will explore this further with additional effects and the development of processing maps predicted through multiple regression. 


\section{Chapter 4:4.1}

\section{THE EFFECT OF SUGAR ON PROCESSING INTERACTIONS AND OPTIMAL PROCESS VALUES IN PALM OIL}

The relationship between microscopic and macroscopic properties in fat-continuous dispersions is multifaceted compared to bulk oils, which limits the ability to extrapolate results from bulk systems towards complex formulations. The impact of confectioner's sugar on response towards cooling rate and endpoint temperature was investigated in this chapter. Adding sugar significantly increased $\mathrm{G}^{\prime}$ and $\mathrm{F}$ of the oils while exhibiting increased sensitivity towards processing conditions. Multiple regression analysis was used to create predictive models that correct for the effects caused by confectioner's sugar, such as altered fat crystal morphology and increased network rigidity, through the binary variable $\zeta$. Furthermore, processing maps were generated according to these models from which optimal process values could be derived.

\footnotetext{
4.1 This chapter has been submitted for publication under the following citation: West, R. and Rousseau, D. (2018). Regression modelling of the impact of sugar and temperature on palm oil crystallization and rheology. Food Chem. (under review).
} 


\subsection{Introduction}

PO is a particularly important ingredient for the confectionery industry as its natural semisolid consistency enables replacement of PHOs in consumer products (Aftab et al., 2013). It is free of trans fats, relatively inexpensive, and does not readily oxidize compared to other fats and oils (Sherazi et al., 2009). Its compositional diversity enables it to be fractionated for use in a wide range of application (Kellens et al., 2007).

Processing conditions will greatly impact the physical properties of bulk oils such as crystallization kinetics and rheology (Sato, 2001; Kellens et al., 2007; Pérez-Martínez et al., 2007). For example, rapid cooling will enhance the extent and rate of nucleation of TAGs resulting in the formation of small, unstable crystals that transition into larger crystals and higher-order polymorphs (Metin and Hartel, 2005). Fat crystal stability (i.e., polymorphism) is also affected by the temperature gradient and endpoint to which a bulk oil is cooled. For example, as observed with $\mathrm{PO}$, processing temperatures above $20{ }^{\circ} \mathrm{C}$ favour the formation of $\beta^{\prime}$ crystals, whereas unstable $\alpha$ crystals begin to form at lower temperatures, resulting in a solid-state $\alpha \rightarrow \beta^{\prime}$ transition during storage (Fernandes et al., 2013).

Fats are rarely present as a bulk material in processed foods. Rather, they often act as a continuous matrix that incorporates non-fat ingredients (e.g., sugar). By confining and compartmentalizing the fat, dispersed ingredients may alter its crystallization pathway and rigidity (Walstra, 2003; Fernandes et al., 2013), with possible consequences on process optimization and sensory properties (Beckett, 2000).

In the previous chapters, it was shown that the relationship between the microscopic and macroscopic properties of $\mathrm{PO}$ and PMF were altered by the presence of confectioner's sugar. 
It was determined that the ability to extrapolate findings from bulk studies towards confectionery formulations was impractical. This chapter explored the effect of processing parameters (i.e., cooling rate and endpoint deviation) on the physical properties of these oils in bulk or blended with confectioner's sugar over four weeks. Regression models were generated to create process maps and establish optimal process values that predict maximum responses for $\Delta \mathrm{H}_{\mathrm{T}}, \mathrm{G}^{\prime}$, and $\mathrm{F}$ using cooling rate, endpoint deviation, and storage time as independent variables. The binary variable $\zeta$ (i.e., 0 or 1 ) was established to account for the effect of confectioner's sugar and consider dispersion-dependent parameters.

\subsection{Materials and Methods}

\subsubsection{Sample Preparation}

Commercial PO and PMF (IOI Loders Croklaan, Channahon, IL, USA) were used in this study. PMF was blended at $65 \%(\mathrm{w} / \mathrm{w})$ with CO (Bunge North America, St. Louis, MS, USA) to obtain similar melting properties as PO according to AOCS Official Method Cd 16b-93 (Figure 2.1) (Firestone, 1998). TAG and DAG compositions of oils were determined (Table 2.1) as described in Section 2.2.2. Oils were processed either as bulk or combined with sieved 6-x confectioner's sugar (Domino Foods Inc., Yonkers, NY, USA) at $50 \%$ (w/w) using a stand mixer (Hobart Canada Inc., Toronto, ON, Canada). The confectioner's sugar contained $3 \%(\mathrm{w} / \mathrm{w})$ starch, $0.5 \%(\mathrm{w} / \mathrm{w})$ moisture, and possessed an average particle size of $30 \mu \mathrm{m}$.

The samples were heated to $60^{\circ} \mathrm{C}$ for $30 \mathrm{~min}$ at $100 \mathrm{rpm}$ in a scraped-surface jacketed kettle (Tenon Engineering Ltd., Leatherhead, UK) connected to a dual bath setup (VWR International 1157P, Mississauga, ON, Canada and Lauda RMS-20, Delran, NJ, USA) to 
eliminate crystal memory. Samples were then cooled $(q)$ at 1,5 , or $10{ }^{\circ} \mathrm{C} \cdot \mathrm{min}^{-1}$ to a temperature of 15,20 , or $28{ }^{\circ} \mathrm{C}$. When cooled to either 15 or $28{ }^{\circ} \mathrm{C}$, a thin layer of sample was transferred to a baking sheet and either heated or cooled in an incubator set to either 40 or $0{ }^{\circ} \mathrm{C}$, respectively, until a thermocouple read $20{ }^{\circ} \mathrm{C}$. Because $20{ }^{\circ} \mathrm{C}$ is the standard endpoint for processing, this effect of altering final temperature is described as $\Delta T$, according to Equation 3.1. Samples were characterized within 15 min upon reaching $20^{\circ} \mathrm{C}$ (i.e., 0 weeks) or stored at $20^{\circ} \mathrm{C}$ and sampled weekly for 4 weeks. All samples were prepared in triplicate.

\subsubsection{Differential Scanning Calorimetry}

DSC (Q2000 and Universal Analysis 2000 software, TA Instruments, New Castle, DE, USA) was used to determine $\Delta \mathrm{H}_{\mathrm{T}}$ of bulk oil and oil-sugar blends. Samples masses $(\sim 10$ mg) were corrected for presence of sugar, if necessary. After equilibrating the sample at 20 ${ }^{\circ} \mathrm{C}$ for $5 \mathrm{~min}$, the temperature was increased to $90{ }^{\circ} \mathrm{C}$ at $5{ }^{\circ} \mathrm{C} \cdot \mathrm{min}^{-1}$, and endotherms corresponding to the $\beta^{\prime}\left(35.3 \pm 1.8^{\circ} \mathrm{C}\right)$ and/or $\beta$ crystals $\left(42.6 \pm 0.9^{\circ} \mathrm{C}\right)$ were identified (Busfield and Proshogo, 1990; Braipson-Danthine and Gibon, 2007). Samples were placed in open aluminum pans for all runs. Nitrogen gas was used to prevent water condensation.

\subsubsection{Microscopy}

CLSM (LSM800 and LSM510, Zeiss Inc., Toronto, ON, Canada) was performed to characterize the impact of processing and the presence of sugar on PO and PMF fat crystal morphology. Fluorol yellow 088 (Sigma-Aldrich, St. Louis, MO, USA) was added to samples at $0.05 \%(\mathrm{w} / \mathrm{w})$ immediately after processing to negatively-stain the fat crystals. Thin layers of sample were placed between a glass slide and cover slip with minimal 
compression and imaged at $630 \times$ using a $63 \times$ oil immersion objective lens paired with a $10 \times$ ocular lens. The built-in imaging software ZEN 2.3 (Zeiss Inc., Toronto, ON, Canada) was used to assess fat crystal morphology and size.

\subsubsection{Rheology}

$\mathrm{G}^{\prime}$ was measured using a strain-controlled rheometer (AntonPaar Physica MCR 301, St. Laurent, QC, Canada) and PP25/P2 serrated geometry where frequency sweep tests within the linear viscoelastic region were performed at $0.01 \%$ strain (i.e., 21 points of $\omega$ were collected between 4 and $\left.63 \mathrm{rad} \cdot \mathrm{sec}^{-1}\right) \cdot{ }^{4.2}$ All rheological tests were performed at $20^{\circ} \mathrm{C}$ with a gap size of $1.0 \mathrm{~mm}$.

\subsubsection{Texture Analysis}

F was measured using a texture analyzer (TA.XTplus, Stable Micro Systems, Ltd., Surrey, UK) calibrated with a $30 \mathrm{~kg}$ load cell. Sixty ml plastic cups filled with bulk oil or oil-sugar blends were stored as above. For analysis, a $45^{\circ}$ steel cone was calibrated to a $10 \mathrm{~mm}$ height above the sample surface and programmed for approach at $2 \mathrm{~mm} \cdot \mathrm{s}^{-1}$. Upon contact with its surface, the cone penetrated the sample at $1 \mathrm{~mm} \cdot \mathrm{s}^{-1}$ for $15 \mathrm{~mm}$ at which point $\mathrm{F}$ was measured.

\subsubsection{Statistical Analysis and Multiple Regression}

A six-way ANOVA from a mixed model using replicate, oil, sugar, cooling rate, endpoint deviation, and storage time (including interactions) was performed to test for significance

\footnotetext{
${ }^{4.2}$ Raw frequency sweep tests for $\mathrm{G}^{\prime}$ are provided in A1.
} 
$(\mathrm{p}<0.05)$ with SAS 9.4 (SAS Institute, Cary, NC, USA). ${ }^{4.3}$ Confectioner's sugar was treated as a binary variable where $\zeta=0$ in bulk systems and $\zeta=1$ in oil-sugar blends. Experimentspecific variables were used as needed. Insignificant variables were removed from these models and their degrees of freedom added back into error.

Pearson correlation coefficients $(\rho)$ were generated to determine the effect of $\zeta$ on the relationship between $\mathrm{G}^{\prime}$ and $\mathrm{F}$ as well as the relationship between observed and predicted properties. Oil-specific third-order polynomial models with parameters for cooling rate, endpoint deviation, and storage time as independent variables were determined using multiple regression analysis — and further corrected for overfitting — as described in Section 3.2.7. Model limitations such as the inability to extrapolate using processing conditions outside of what was performed experimentally must be acknowledged. Furthermore, $\zeta$ cannot be used as a continuous variable [e.g., $\zeta=0.5$ to predict the effect of $25 \%(\mathrm{w} / \mathrm{w})$ confectioner's sugar on an oil-sugar blend] as this presumes the existence of a linear effect of confectioner's sugar on oil response. Ultimately, these models were used to generate process maps as well as optimal process values. ${ }^{4.4}$

\subsection{Results and Discussion}

\subsubsection{Total Enthalpy and Polymorphism}

The main effects of composition, processing, and storage on $\Delta \mathrm{H}_{\mathrm{T}}$ of the bulk oil and oilsugar blends are depicted in Figure 4.1. In general terms, the addition of confectioner's sugar

\footnotetext{
4.3 ANOVA tables are listed in A2.3.

${ }^{4.4}$ Mathematical description of confidence limits associated with maximum response provided in A3.2.
} 

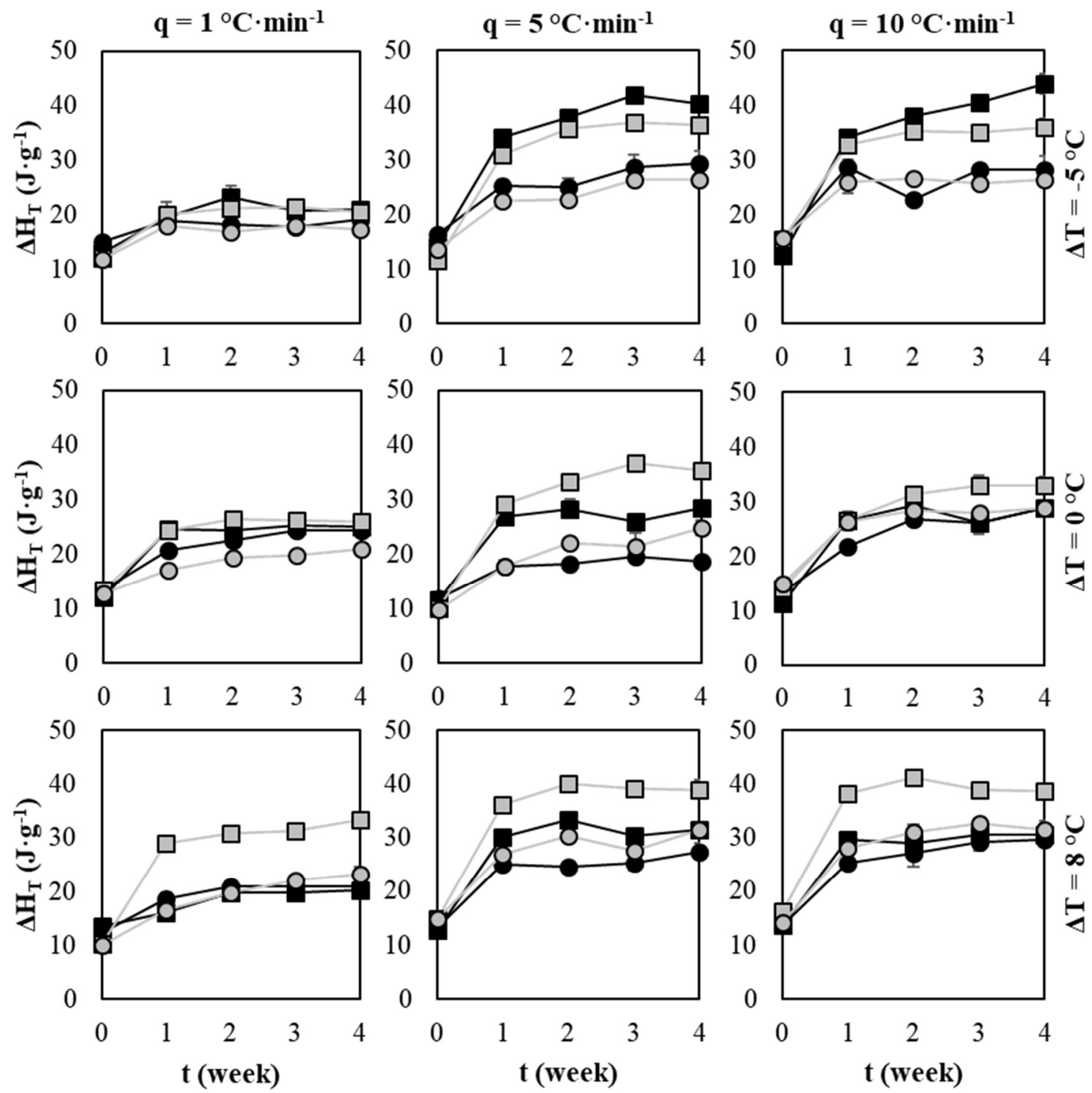

$\rightarrow \mathrm{PO}, \zeta=0 \rightarrow \mathrm{PMF}, \zeta=0 \quad \square-\mathrm{PO}, \zeta=1 \quad \mathrm{O}-\mathrm{PMF}, \zeta=1$

Figure 4.1 Effects of sugar and processing conditions on $\triangle H_{T}$ of $P O$ and $P M F$ over time.

either had no effect or increased $\Delta \mathrm{H}_{\mathrm{T}}$, particularly with PO and higher cooling rates, except when cooled to $15^{\circ} \mathrm{C}$ where a suppression was observed (Figure 4.1). For example, whereas the addition of sugar to $\mathrm{PO}$ cooled at $10{ }^{\circ} \mathrm{C} \cdot \mathrm{min}^{-1}$ increased final $\Delta \mathrm{H}_{\mathrm{T}}$ (i.e., week 4 ) by 4.2 and $8.0 \mathrm{~J} \cdot \mathrm{g}^{-1}$ when cooled to 20 and $28^{\circ} \mathrm{C}$, respectively, a reduction of $7.9 \mathrm{~J} \cdot \mathrm{g}^{-1}$ was observed when cooled to $15^{\circ} \mathrm{C}$. 
With the results of all experimental processing conditions combined, the $\Delta \mathrm{H}_{\mathrm{T}}$ of bulk PO, which was composed of more 1,2-PO, 1,3-PO, 1,3-PL and whose overall DAG content was $2.3 \%$ higher than PMF, was $1.2 \mathrm{~J} \cdot \mathrm{g}^{-1}$ lower than PMF. While DAGs have been suggested to delay crystallization, this mechanism has not been fully elucidated (Tshiamala, 2013). The initial $\Delta \mathrm{H}_{\mathrm{T}}$ of PO-sugar blends increased from $11.9 \pm 1.6$ to $12.1 \pm 2.2$ to $15.3 \pm 1.5$ $\mathrm{J} \cdot \mathrm{g}^{-1}$ (i.e., averaged across $\Delta \mathrm{T}$ ) with increasing cooling rate. For PMF-sugar blends, initial $\Delta \mathrm{H}_{\mathrm{T}}$ increased from $11.6 \pm 1.2$ to $12.8 \pm 2.2$ to $14.9 \pm 0.9 \mathrm{~J} \cdot \mathrm{g}^{-1}$. Hence, the increase observed in both oil-sugar blends was similar.

The $\Delta \mathrm{H}_{\mathrm{T}}$ of bulk PO was greater than PMF after one week of storage, which was attributed to its higher palmitic-rich disaturated [e.g., $\mathrm{P}(\mathrm{PO}), \mathrm{P}(\mathrm{PL})$, and (POS)] and monosaturated [e.g., O(PO), (PLO), L(PL)] TAG and lower unsaturated [e.g., OOO, O(LO), L(LO)] TAG content (Table 2.1). Furthermore, the increase in final $\Delta \mathrm{H}_{\mathrm{T}}$ due to sugar was greater in $\mathrm{PO}$ compared to PMF. For example, whereas PMF had increased by 0.9 and $2.8 \mathrm{~J} \cdot \mathrm{g}^{-1}$ (i.e., averaged across q) when cooled to 20 and $28{ }^{\circ} \mathrm{C}$, respectively, PO increased by 3.9 and 9.5 $\mathrm{J} \cdot \mathrm{g}^{-1}$ (Figure 4.1).

DSC is often used to indirectly identify fat crystal polymorph type. The present results demonstrated that both temperature profile and addition of sugar greatly altered the overall melting profile as well as relative contributions of $\beta^{\prime}$ and $\beta$ crystals. Although high cooling rates typically induce the formation of unstable crystals (e.g., lower-order polymorphs), the thermograms did not identify such trends. Most interesting was that $\beta$ crystals did not form in the presence of sugar apart from oils processed at high cooling rates to $28^{\circ} \mathrm{C}$ (Figure 4.2). This indicated the ability of sugar to facilitate the desirable formation of $\beta^{\prime}$ crystals in PO 

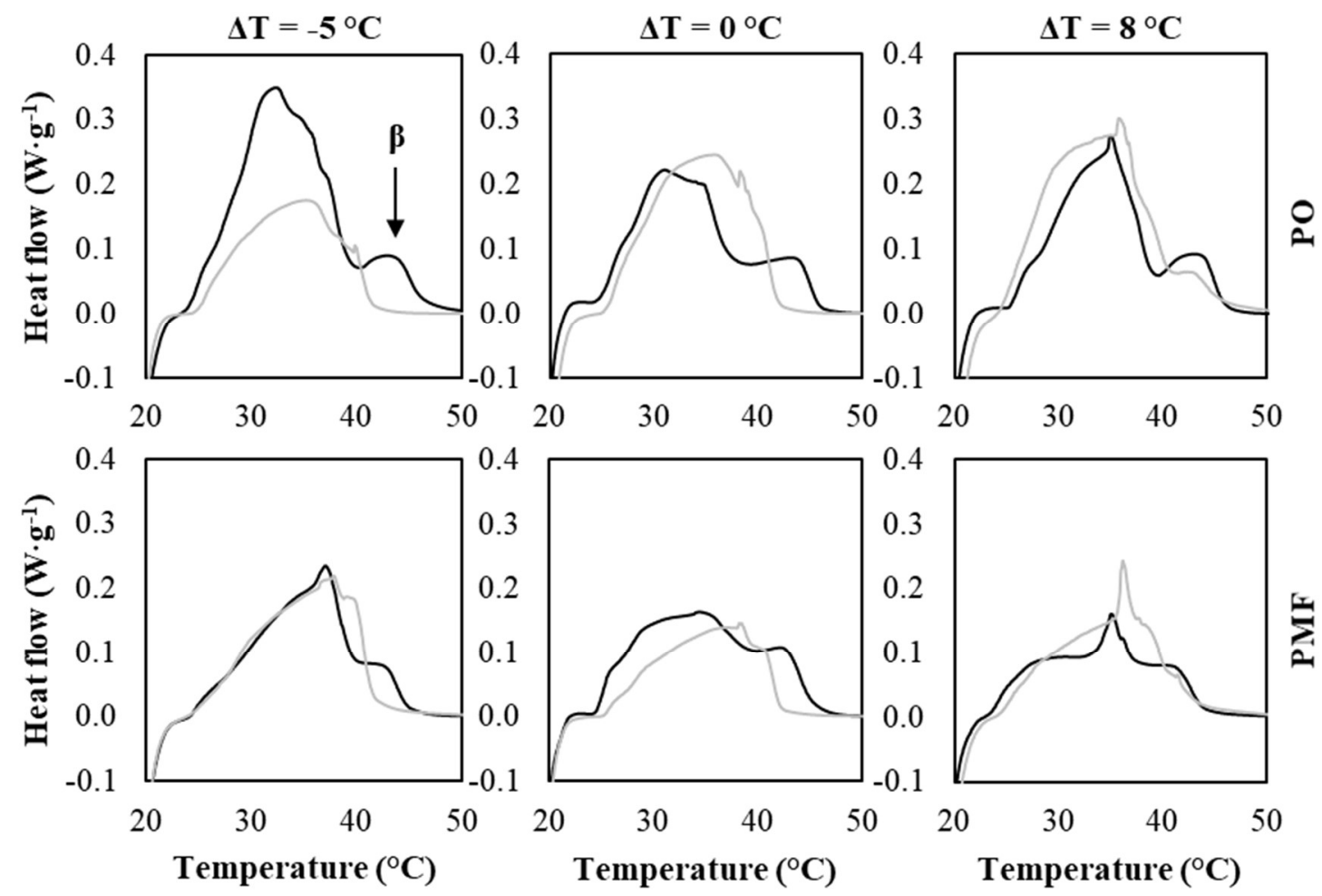

$-\zeta=0-\zeta=1$

Figure 4.2 Impact of sugar and endpoint on polymorphic composition of PO and PMF cooled at $10^{\circ} \mathrm{C} \cdot \mathrm{min}^{-1}$.

with most processing conditions. Also of interest was the existence of a third endotherm at $24.0 \pm 0.9^{\circ} \mathrm{C}$ when the oils were cooled to $15^{\circ} \mathrm{C}$, which may suggest the presence of $\alpha$ crystals (Fernandes et al., 2013).

The relationship evident between the final $\Delta \mathrm{H}_{\mathrm{T}}$ of the oil-sugar blends and cooling rate suggested the presence of an optimal cooling rate $\left(q_{0}\right)$ (Table 4.1), which was determined from subset models: 
Table 4.1 Optimal process values and associated maximum response at week 4.

\begin{tabular}{|c|c|c|c|c|c|c|c|}
\hline \multirow{2}{*}{ Response } & \multirow{2}{*}{$\begin{array}{c}\text { Oil } \\
\text { (Model) }\end{array}$} & \multicolumn{3}{|c|}{ Optimal process values } & \multirow{2}{*}{$\hat{y}_{\max }$} & \multicolumn{2}{|c|}{ Confidence limits (95\%) } \\
\hline & & s & $q_{0}\left({ }^{\circ} \mathrm{C} \cdot \mathrm{min}^{-1}\right)$ & $\Delta T_{0}\left({ }^{\circ} \mathrm{C}\right)$ & & Lower & Upper \\
\hline \multirow{4}{*}{$\Delta \mathrm{H}_{\mathrm{T}}\left(\mathrm{J} \cdot \mathrm{g}^{-1}\right)$} & $\mathrm{PO}$ & 0 & 8.4 & -5.0 & 44.5 & 42.8 & 46.3 \\
\hline & (4.1a) & 1 & 6.5 & 8.0 & 42.2 & 40.5 & 43.8 \\
\hline & PMF & 0 & 10.0 & 8.0 & 30.5 & 28.6 & 32.3 \\
\hline & $(4.1 b)$ & 1 & 8.1 & 8.0 & 32.5 & 30.9 & 34.1 \\
\hline \multirow{4}{*}{$\mathrm{G}^{\prime}(\mathrm{MPa})$} & $\mathrm{PO}$ & 0 & 5.1 & -0.2 & 4.0 & 3.8 & 4.2 \\
\hline & $(4.2 \mathrm{a})$ & 1 & 6.2 & -1.7 & 8.3 & 8.1 & 8.4 \\
\hline & PMF & 0 & 10.0 & -5.0 & 2.7 & 2.6 & 2.8 \\
\hline & $(4.2 b)$ & 1 & 7.7 & -2.1 & 5.7 & 5.6 & 5.8 \\
\hline \multirow{4}{*}{$\mathrm{F}(\mathrm{kg})$} & $\mathrm{PO}$ & 0 & 8.4 & 4.6 & 1.7 & 1.5 & 1.9 \\
\hline & (4.3a) & 1 & 1.0 & 6.9 & 2.9 & 2.6 & 3.1 \\
\hline & PMF & 0 & 10.0 & -5.0 & 1.1 & 1.0 & 1.2 \\
\hline & $(4.3 b)$ & 1 & 7.1 & 8.0 & 1.7 & 1.6 & 1.8 \\
\hline
\end{tabular}

$$
\begin{aligned}
\hat{\mathrm{y}}=13.8868+ & 0.2597 \Delta T+15.6970 t-0.1064 \Delta T^{2}-8.0355 t^{2} \\
& -0.0057 q^{3}+1.0831 t^{3}-0.0805 q \Delta T+1.5540 q t \\
& -0.2140 \Delta T t-0.0732 q^{2} t+0.0233 \Delta T^{2} t+0.0276 q \Delta T^{2} \\
& -0.1056 q t^{2}+0.0349 \Delta T t^{2}-0.0244 q \Delta T t \\
& +\zeta\left(-1.9874+3.3392 t+0.0494 q^{2}+0.0646 \Delta T^{2}\right. \\
& -0.4208 t^{2}+0.6540 \Delta T t+0.0042 q^{2} \Delta T-0.0142 q^{2} t \\
& \left.-0.0217 \Delta T^{2} t-0.0110 q \Delta T^{2}-0.0974 \Delta T t^{2}\right)
\end{aligned}
$$

$\left(C_{P}=22.61, \mathrm{~F}_{26,243}=141.48, \mathrm{p}<0.0001\right)$ for $\mathrm{PO}$ and:

$$
\begin{aligned}
\hat{\mathrm{y}}=13.2123 & -0.2271 \Delta T+9.8032 t-4.4405 t^{2}+0.6221 t^{3} \\
& -0.0303 q \Delta T+0.3344 q t+0.2387 \Delta T t+0.0083 q \Delta T^{2} \\
& -0.0488 q t^{2}-0.0389 \Delta T t^{2} \\
& +\zeta(-2.6758+0.3515 q+0.1254 q \Delta T+0.5382 q t \\
& \left.-0.0086 q^{2} \Delta T-0.0331 q^{2} t-0.0037 q \Delta T^{2}-0.0538 q t^{2}\right)
\end{aligned}
$$

$\left(C_{P}=8.96, \mathrm{~F}_{18,251}=70.98, \mathrm{p}<0.0001\right)$ for PMF, respectively. Hence, optimal rates of 6.5 and $8.1{ }^{\circ} \mathrm{C} \cdot \mathrm{min}^{-1}$ were recommended for PO- and PMF-sugar blends from the models, respectively, to obtain maximum $\Delta \mathrm{H}_{\mathrm{T}}$ values after 4 weeks. Optimal endpoint deviations 
$\left(\Delta T_{0}\right)$ of $8.0^{\circ} \mathrm{C}$ were recommended for either blend to obtain this value. Process maps of predicted $\Delta \mathrm{H}_{\mathrm{T}}$ based on these models are illustrated in Figure 4.3.

\subsubsection{Storage Modulus and Fat Crystal Morphology}

The rheology of fats and oils is influenced by factors such as the extent of fat solidification, crystal morphology, and spatial distribution (Pérez-Martínez et al., 2007). Not only is rheology used to indicate product quality, it can also give an indication of production efficiency (Kilcast and Clegg, 2002). The presence of a dispersed phase, whether solid, liquid, or gas, may confound any association between rheology and other properties of bulk oils by imparting additional interactions and altering TAG crystallization pathway (Rao, 2007).

The main effects of composition, processing, and storage time on $\mathrm{G}^{\prime}$ are depicted in Figure 4.4. The addition of confectioner's sugar increased $\mathrm{G}^{\prime}$ after storage regardless of oil or processing conditions. While volume fraction and interparticle interactions can alter the rheology of colloidal suspensions (Trappe and Weitz, 2000), the direct contribution of confectioner's sugar towards G' (i.e., false positive) was negligible, as described previously in Chapter 2. In that respect, the increase in $\mathrm{G}^{\prime}$ upon addition of sugar resulted from differences in total solidification (i.e., $\Delta \mathrm{H}_{\mathrm{T}}$ ) and fat crystal morphology. Furthermore, addition of confectioner's sugar to fat at $50 \%(\mathrm{w} / \mathrm{w})$ may impact spatial distribution and rigidity as fat must crystallize in a system where total space is reduced by $37 \%(\mathrm{v} / \mathrm{v})$ to accommodate this dispersed phase.

Although PO exhibited higher $\mathrm{G}^{\prime}$ values than PMF from weeks 1 to 4 regardless of the 

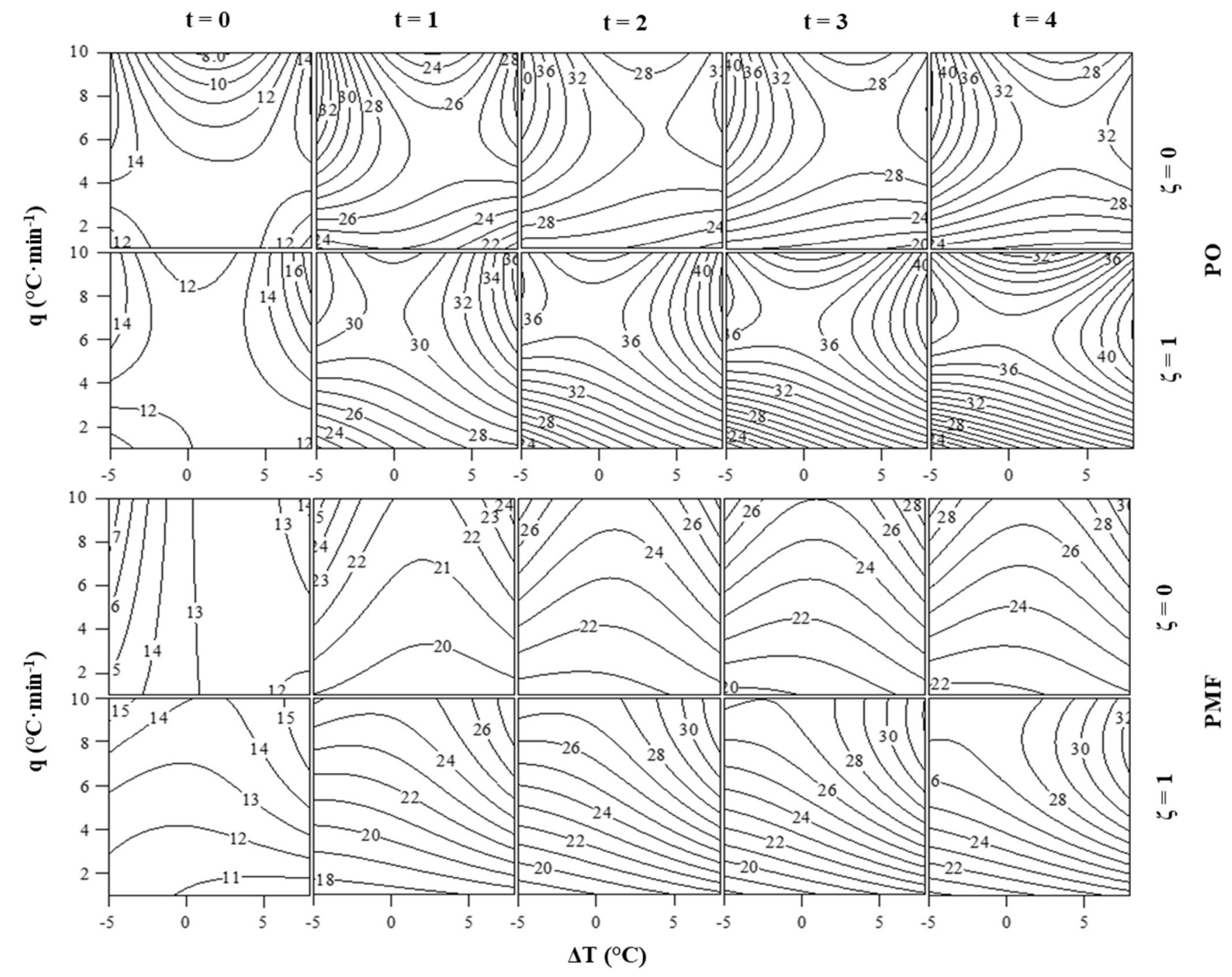

Figure 4.3 Predicted process maps of $\Delta H_{T}\left(J \cdot g^{-1}\right)$ according to models 4.1 a and $4.1 \mathrm{~b}$. 

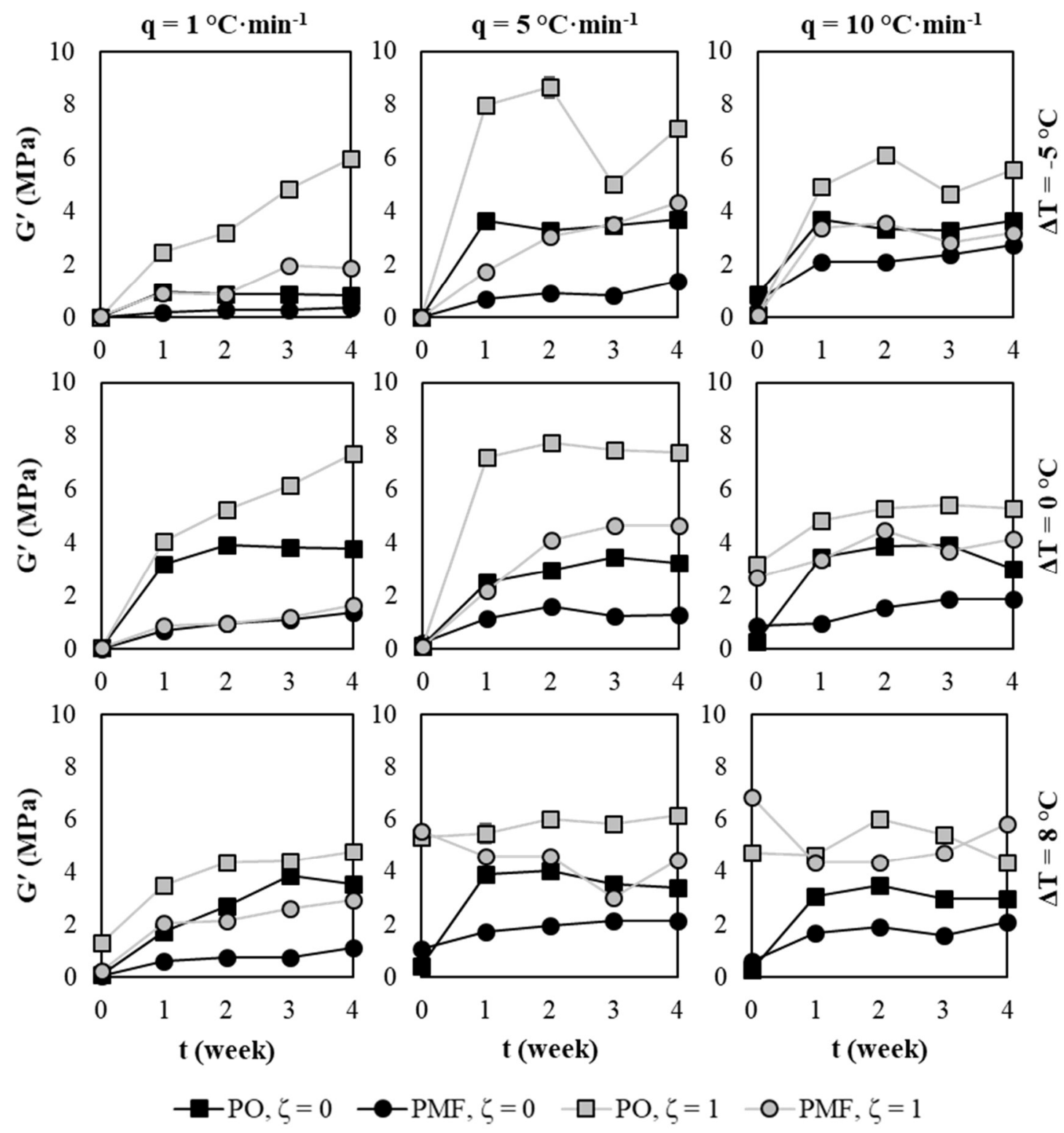

Figure 4.4 Effects of sugar and processing conditions on $G^{\prime}$ of $P O$ and PMF over time.

presence of confectioner's sugar or processing conditions imposed, its initial $\mathrm{G}^{\prime}$ was usually lower. This was especially apparent in the bulk oils and corresponded to the lower initial $\Delta \mathrm{H}_{\mathrm{T}}$ observed (Figure 4.1). The extent of the difference in $\mathrm{G}^{\prime}$ between PO and PMF became greater with time, which was explained both by its higher degree of FA saturation (Table 2.1) and $\Delta \mathrm{H}_{\mathrm{T}}$ following storage. 

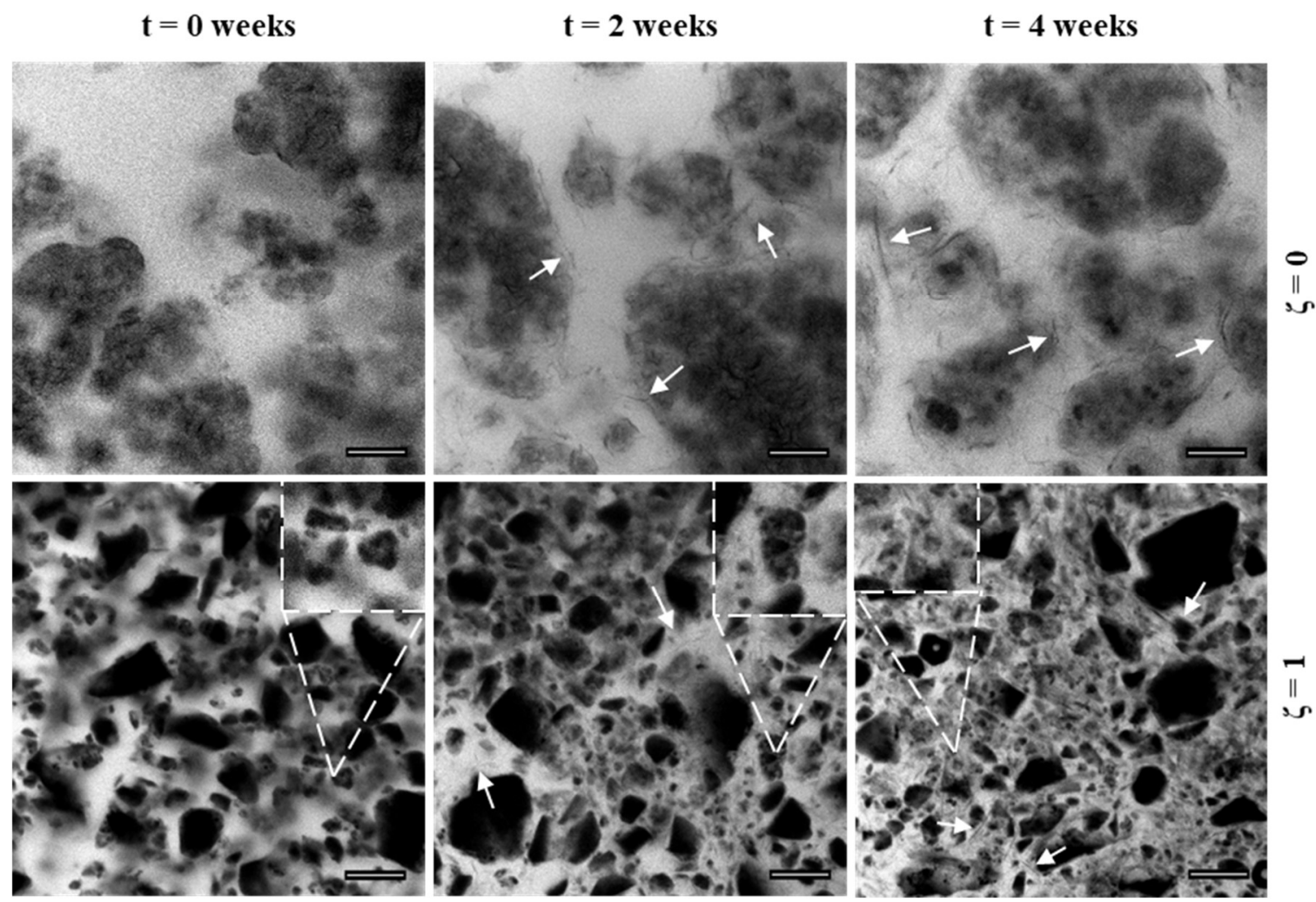

Figure 4.5 Effects of sugar and storage on PO crystal morphology processed under standard conditions (bar $=20 \mu \mathrm{m})$.

The fat crystal morphology of PO processed using standard processing conditions (i.e., cooled at $1{ }^{\circ} \mathrm{C} \cdot \mathrm{min}^{-1}$ to $20^{\circ} \mathrm{C}$ ) revealed the initial presence of agglomerated, spheroidal fat crystal assemblies that increased in size and extent of clustering upon storage. After one week, thin needle-like crystals were apparent (Figure 4.5, indicated by arrows), which was ascribed to secondary nucleation and growth of TAGs not incorporated within the initial assemblies.

The initial fat crystal size of PO was significantly reduced in the presence of confectioner's sugar (visible as the black objects in Figure 4.5). Two factors explained these results. First, given the mixing that occurred during processing, the sugar effectively milled the growing fat crystals such that their post-processing morphology was small and smooth, as evidenced 
in Figure 4.5. Importantly, there was no indication of TAG nucleation off the surface of the confectioner's sugar, which implied that it did not enhance crystallization. Whereas large spheroidal clusters $(\leq 45 \mu \mathrm{m})$ were observed in bulk PO by week 4 , only tiny, smoother crystals $(\leq 11 \mu \mathrm{m})$ formed in the oil-sugar blends, with no evidence of the sugar crystals directly interacting with the TAGs. Restricted crystal growth was amplified by the limited physical space available, as newly-formed fat crystals were now forced to grow within a confined space given the significant volume fraction occupied by the dispersion. Upon storage, the fat crystal network in the oil-sugar blends was composed of tiny, needle-shaped crystals (Figure 4.5, arrows). Interestingly, there appeared to be incorporation of smaller sugar particles within these fat crystal assemblies (Figure 4.5, inset).

As noted in Figure 4.1, addition of confectioner's sugar did not impact $\Delta \mathrm{H}_{\mathrm{T}}$ of PO processed under standard conditions, where both the bulk oil and oil-sugar blends saw plateau values reached after one week of storage. Rheology trends for similar-processed PO were markedly different as the $\mathrm{G}^{\prime}$ of bulk PO also reached a plateau value after one week. By contrast, the PO-sugar blend saw its $\mathrm{G}^{\prime}$ significantly increase with time (Figure 4.4). This was likely explained by the continued sintering of the crystalline network within the now-restricted space for growth created by sugar. A noteworthy result was the increase in $\mathrm{G}^{\prime}$ due to sugar when PO was processed at $10^{\circ} \mathrm{C} \cdot \min ^{-1}$ to $15^{\circ} \mathrm{C}$ (i.e., $\Delta T=-5^{\circ} \mathrm{C}$ ) despite a significant loss in corresponding $\Delta \mathrm{H}_{\mathrm{T}}$. Whereas the addition of sugar suppressed final $\Delta \mathrm{H}_{\mathrm{T}}$ from $43.9 \pm 3.1$ to $36.0 \pm 2.8 \mathrm{~J} \cdot \mathrm{g}^{-1}$ (an $18 \%$ drop) (Figure 4.1 ), the final $\mathrm{G}^{\prime}$ rose from $3.7 \pm 0.5$ to $5.5 \pm 1.3$ MPa (a 49\% increase) (Figure 4.4). This may be partially explained by the impact that dispersions have on spatial distribution and rigidity. 


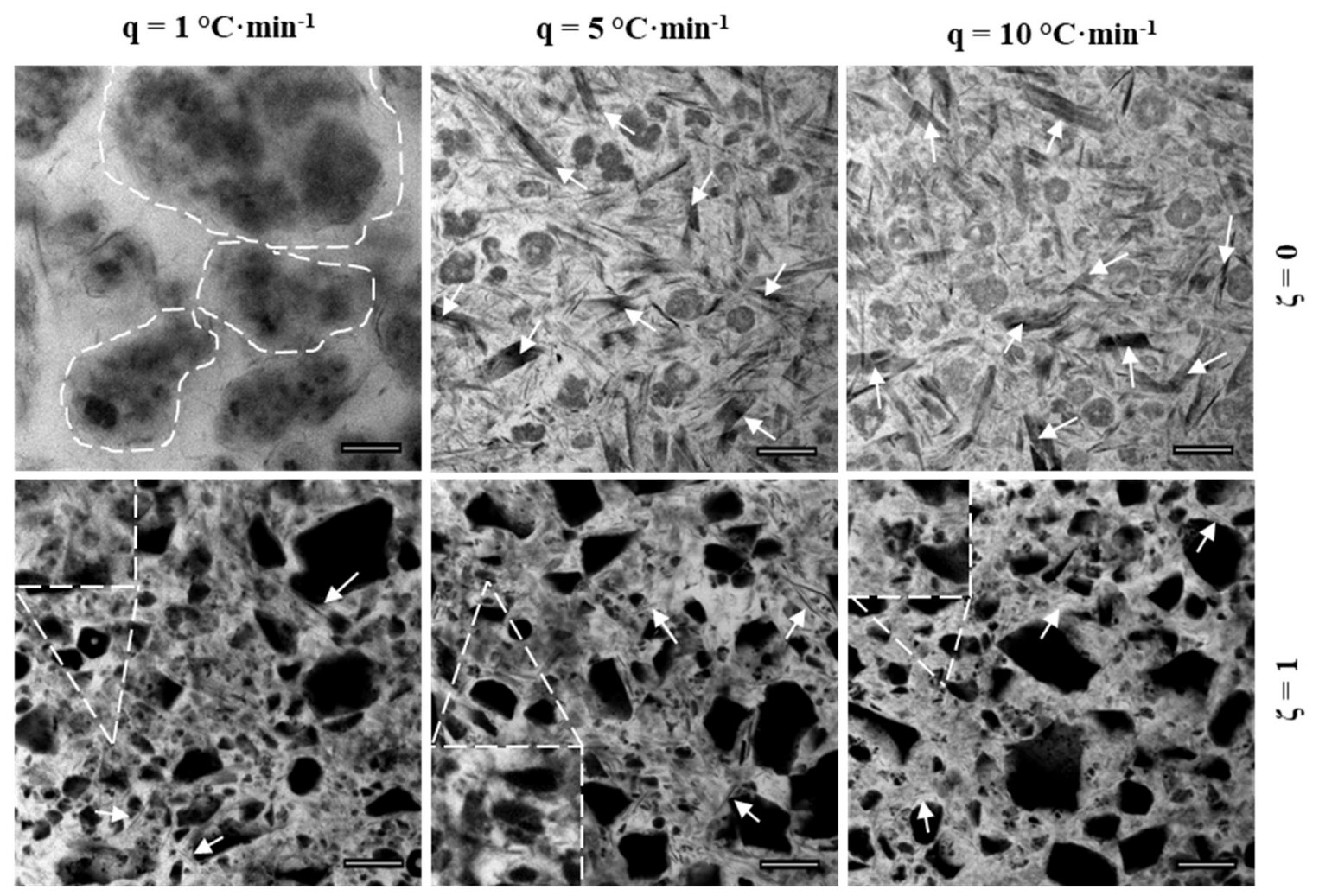

Figure 4.6 Effects of sugar and cooling rate on final PO crystal morphology (e.g., week 4) cooled to $20^{\circ} \mathrm{C}($ bar $=20 \mu \mathrm{m})$.

Fat crystal clustering (Figure 4.6, circles) was reduced in the bulk oils with cooling rates of 5 or $10^{\circ} \mathrm{C} \cdot \mathrm{min}^{-1}$, resulting in smaller, more numerous initial crystals. With time, growth of blade-like crystals in the network occurred, but only in PO cooled at higher rates (Figure 4.6, arrows). Such a morphology has been observed with $\mathrm{CB}$ and is associated with $\beta$ crystals (Manning and Dimick, 1985). As PO is a $\beta^{\prime}$ stable oil, this suggested that fractionation of lower- and higher-melting TAGs under this regime. With the selective crystallization of higher-melting TAGs, the remaining liquid-state TAGs also became more compositionally homogeneous, which resulted in the formation of its own, distinct fat crystal morphology upon storage. PMF did not exhibit blade-like crystals in response to cooling rate, ${ }^{4.5}$ which

\footnotetext{
${ }^{4.5}$ Effects of sugar and cooling rate on PMF crystal morphology shown in A4.
} 
may be a result of its simpler composition and inability to fractionate to the extent of $\mathrm{PO}$ into a mixed crystal system.

A notable evolution of $\mathrm{G}^{\prime}$ was apparent in oil-sugar blends processed at high cooling rates under high cooling rate to $28^{\circ} \mathrm{C}$ (Figure 4.4). For example, the initial $\mathrm{G}^{\prime}$ of PO-sugar blends increased by either 27 - or 40 -fold as cooling rate increased from 1 to either 5 or $10{ }^{\circ} \mathrm{C}$ $\min ^{-1}$, respectively. While initial $\Delta \mathrm{H}_{\mathrm{T}}$ also increased (i.e., $\sim 1.5$-fold), this did not fully explain such large $\mathrm{G}^{\prime}$ observed. Similarly, other processing conditions resulting in higher initial $\Delta \mathrm{H}_{\mathrm{T}}$ values (Figure 4.1) did not exhibit corresponding behaviour in initial $\mathrm{G}^{\prime}$ values. For example, the initial $\mathrm{G}^{\prime}$ of PO-sugar blends cooled at $10^{\circ} \mathrm{C} \cdot \mathrm{min}^{-1}$ increased from $0.1 \pm$ 0.1 to $4.7 \pm 2.0 \mathrm{MPa}$ as the final temperature increased from 15 to $28^{\circ} \mathrm{C}$ (Figure 4.4), yet initial $\Delta \mathrm{H}_{\mathrm{T}}$ differed by just $0.8 \mathrm{~J} \cdot \mathrm{g}^{-1}$ (Figure 4.1). Micrographs of PO-sugar blends cooled to $28{ }^{\circ} \mathrm{C}$ showed evidence of very small crystals not seen with other processing conditions (Figure 4.7, arrows) and may contribute to the large initial $\mathrm{G}^{\prime}$ observed. This may also suggest the ability for fat crystals to nucleate freely within the interstices and independently from any catalytic sites that sugar supplies under supercooling (i.e., high $q$ and $\Delta T$ ) where energy is rapidly removed from the system. In this regard, slower processing conditions (i.e., low $q$ and $\Delta T$ ) may better facilitate nucleation at these interfacial sites, however, this has not been definitively proven via CLSM.

Changes in cooling rate altered $\mathrm{G}^{\prime}$ more extensively in the oil-sugar blends versus their bulk counterpart throughout storage. For example, cooling PO-sugar at either 1 or $5{ }^{\circ} \mathrm{C} \cdot \mathrm{min}^{-1}$ to $20{ }^{\circ} \mathrm{C}$ produced the same final $\mathrm{G}^{\prime}$ (i.e., week 4), however, the time to reach this value was significantly reduced at $5{ }^{\circ} \mathrm{C} \cdot \mathrm{min}^{-1}$ (Figure 4.4 ) ( 1 vs 3 weeks). Of note, the final $\mathrm{G}^{\prime}$ of PO- 


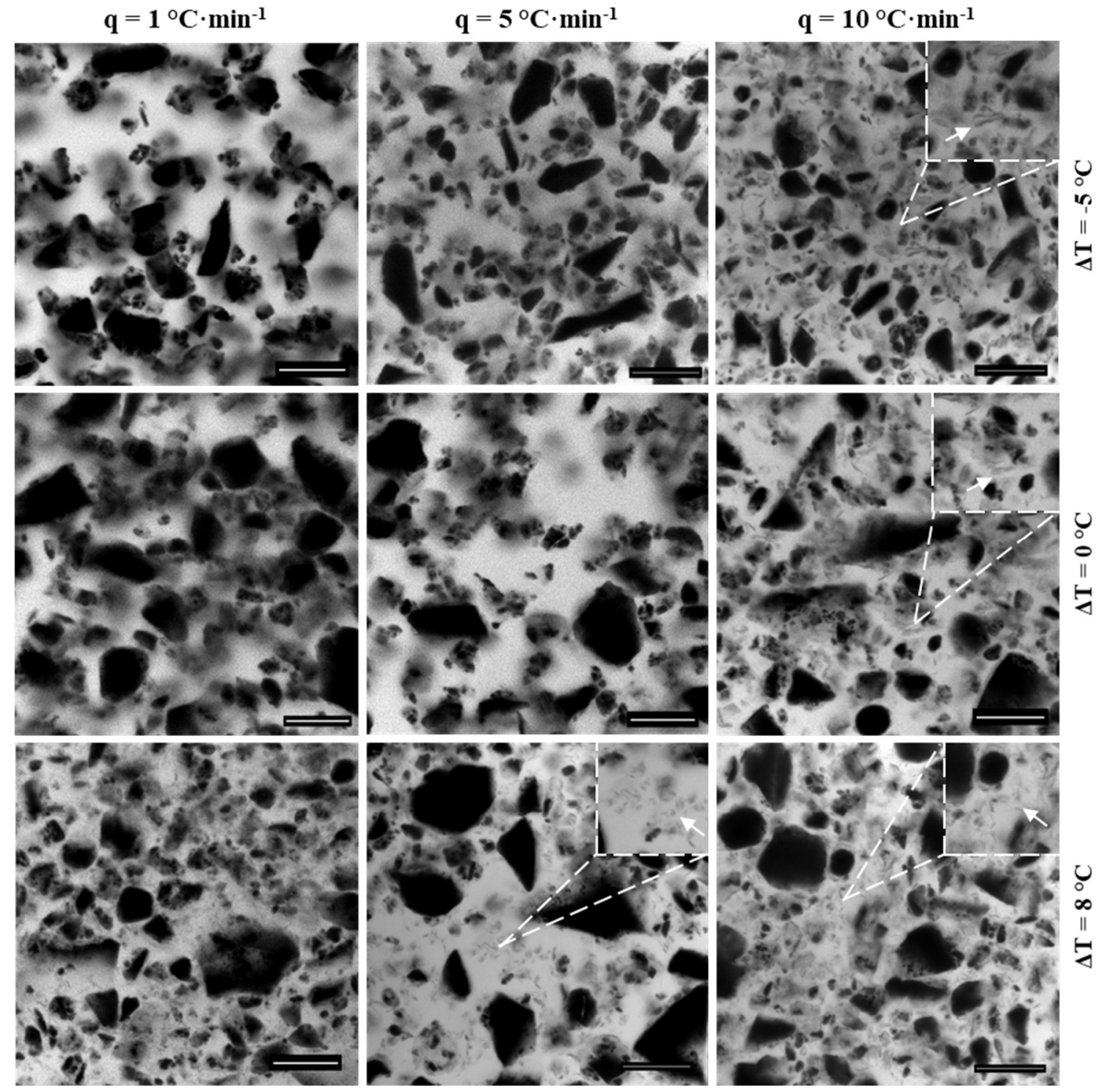

Figure 4.7 Effects of processing on initial crystal morphology (e.g., week 0) of PO-sugar blends (bar $=20 \mu \mathrm{m})$.

sugar decreased from $7.4 \pm 0.5$ to $5.3 \pm 0.6 \mathrm{MPa}$ as cooling rate increased from 5 to 10 ${ }^{\circ} \mathrm{C} \cdot \mathrm{min}^{-1}$, which may be partially explained by the reduction in $\Delta \mathrm{H}_{\mathrm{T}}$ observed (Figure 4.1). With this large decrease in the final $\mathrm{G}^{\prime}$ of PO-sugar at $10{ }^{\circ} \mathrm{C} \cdot \mathrm{min}^{-1}$, an optimal cooling rate of $6.2{ }^{\circ} \mathrm{C} \cdot \mathrm{min}^{-1}$ was suggested to maximize the response of final $\mathrm{G}^{\prime}$ (Table 4.1), according to the subset model: 


$$
\begin{aligned}
\hat{\mathrm{y}}=0.1813+ & 0.1361 q+3.5238 t-0.0142 q^{2}-0.0292 \Delta T^{2}-1.4161 t^{2} \\
& +0.0028 \Delta T^{3}+0.1826 t^{3}-0.0341 q \Delta T+0.0997 q t \\
& +0.0919 \Delta T t+0.0015 q^{2} \Delta T-0.0037 q^{2} t-0.0046 \Delta T^{2} t \\
& +0.0037 q \Delta T^{2}-0.0154 q t^{2}-0.0082 \Delta T t^{2}-0.0056 q \Delta T t \\
& +\zeta\left(-0.3563+2.3372 t+0.1616 q^{2}-0.7209 t^{2}\right. \\
& -0.0144 q^{3}+0.0035 \Delta T^{3}+0.0997 t^{3}+0.0180 q \Delta T \\
& -0.1294 q t-0.2660 \Delta T t+0.0044 q^{2} t-0.0037 \Delta T^{2} t \\
& \left.-0.0012 q \Delta T^{2}+0.0445 \Delta T t^{2}+0.0032 q \Delta T t\right)
\end{aligned}
$$

$\left(C_{P}=32.66, \mathrm{~F}_{32,5585}=463.91, \mathrm{p}<0.0001\right)$. A different response to cooling rate was observed with PMF-sugar blends, which did not exhibit a suppression of final $\mathrm{G}^{\prime}$ as cooling rate increased above $5{ }^{\circ} \mathrm{C} \cdot \mathrm{min}^{-1}$. As a result, the suggested optimal cooling rate for the PMFsugar blend was higher, at $7.7^{\circ} \mathrm{C} \cdot \mathrm{min}^{-1}$, according to the subset model:

$$
\begin{aligned}
\hat{\mathrm{y}}=0.2194+ & 0.8588 t+0.0122 q^{2}-0.0128 \Delta T^{2}-0.3839 t^{2}-0.0010 q^{3} \\
& +0.0004 \Delta T^{3}+0.0554 t^{3}+0.0335 q \Delta T+0.0393 q t \\
& +0.0104 \Delta T t-0.0039 q^{2} \Delta T+0.0023 q \Delta T^{2}-0.0056 q t^{2} \\
& -0.0023 q \Delta T t \\
& +\zeta\left(-0.9845-0.3972 \Delta T+0.9377 t+0.1107 q^{2}\right. \\
& -0.2252 t^{2}-0.0080 q^{3}+0.0115 \Delta T^{3}+0.0198 t^{3} \\
& +0.0102 q \Delta T+0.0447 q t-0.1528 \Delta T t+0.0030 q^{2} \Delta T \\
& -0.0119 q^{2} t-0.0046 \Delta T^{2} t-0.0039 q \Delta T^{2}+0.0125 q t^{2} \\
& \left.+0.0351 \Delta T t^{2}-0.0044 q \Delta T t\right)
\end{aligned}
$$

$\left(C_{P}=31.01, \mathrm{~F}_{32,5611}=808.95, \mathrm{p}<0.0001\right)$, with only a minor loss in final $\mathrm{G}^{\prime}$ if this rate were to be exceeded. Consequently, PMF-sugar blends may be subjected to more rapid thermal processing in terms of cooling rate without concern for diminished $\mathrm{G}^{\prime}$. Process maps of predicted $\mathrm{G}^{\prime}$ according to both models are illustrated in Figure 4.8.

\subsubsection{Firmness}

Texture analysis measures mechanical properties such as F, which can support textural qualities that consumers desire. There is also an inherent relationship between these 


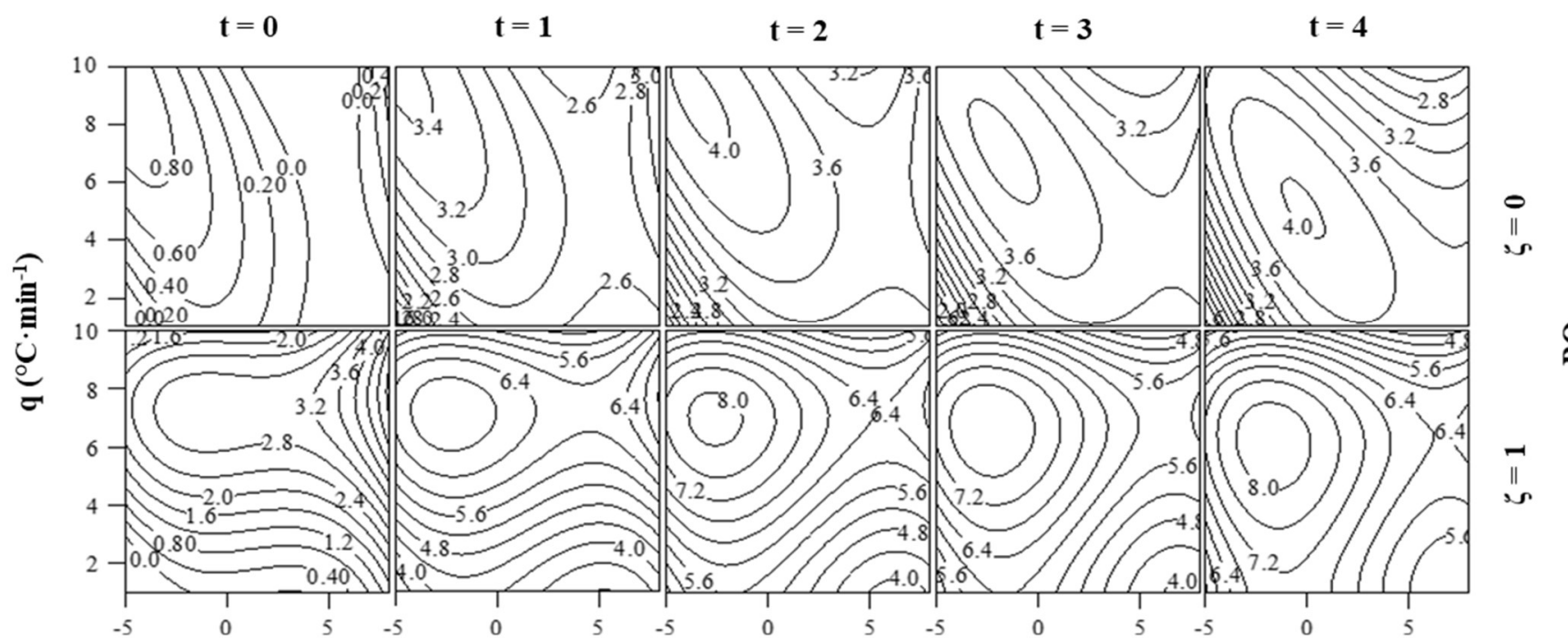

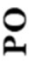

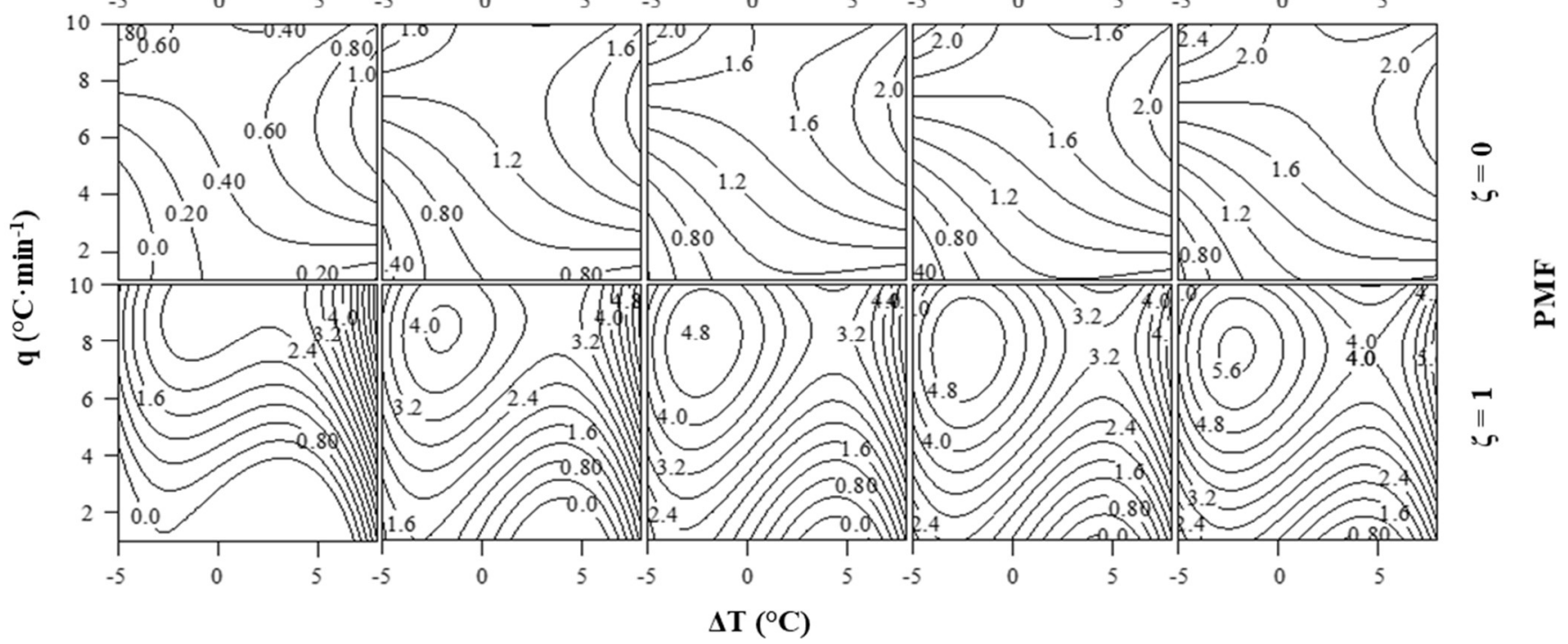

Figure 4.8 Predicted process maps of $G^{\prime}$ (MPa) according to models $4.2 a$ and $4.2 b$. 
mechanical properties and rheology. For example, the relationship between $F, \tau_{0}$, and $\eta$ of dark chocolate has previously been examined through multivariate analysis by Afoakwa et al. (2008).

The addition of confectioner's sugar increased $\mathrm{F}$ regardless of oil or processing conditions (Figure 4.9), with this increase generally greater in PO than PMF. The initial F of either oilsugar blend also increased with cooling rate. For example, PO-sugar blends cooled to $28^{\circ} \mathrm{C}$ increased from $178.0 \pm 36.8$ to $1400.0 \pm 316.5 \mathrm{~g}$ as the cooling rate increased from 1 to 10 ${ }^{\circ} \mathrm{C} \cdot \min ^{-1}$.

Minor differences existed in $\mathrm{F}$ at 4 weeks under most processing conditions despite greater differences observed in $\mathrm{G}^{\prime}$ (Figure 4.4) and fat crystal morphology (Figures 4.6 and 4.7), which resulted in an optimal cooling rate of $1.0^{\circ} \mathrm{C} \cdot \mathrm{min}^{-1}$ (Table 4.1) according to the subset model:

$$
\begin{aligned}
\hat{\mathrm{y}}=0.0260+ & 0.1180 \Delta T+1.7867 t+0.0129 q^{2}-0.7620 t^{2}-0.0012 q^{3} \\
& -0.0017 \Delta T^{3}+0.0942 t^{3}-0.0147 q \Delta T+0.0092 q t \\
& +0.0007 q^{2} \Delta T+0.0008 q \Delta T^{2} \\
& +\zeta(0.0301 q+0.3473 t+0.0104 q \Delta T-0.0280 q t \\
& \left.-0.0204 \Delta T t+0.0079 \Delta T t^{2}-0.0030 q \Delta T t\right)
\end{aligned}
$$

$\left(C_{P}=11.49, \mathrm{~F}_{18,251}=80.77, \mathrm{p}<0.0001\right)$, with observed differences likely under consumer detectability. This resistance in final F towards final temperature may benefit industry as initial physical properties can be manipulated to improve flowability and production efficiency while final properties remain unchanged. For PMF-sugar blends, an optimal cooling rate of $7.1^{\circ} \mathrm{C} \cdot \mathrm{min}^{-1}$ was suggested for maximum final $\mathrm{F}$ (Table 4.1), according to the subset model: 

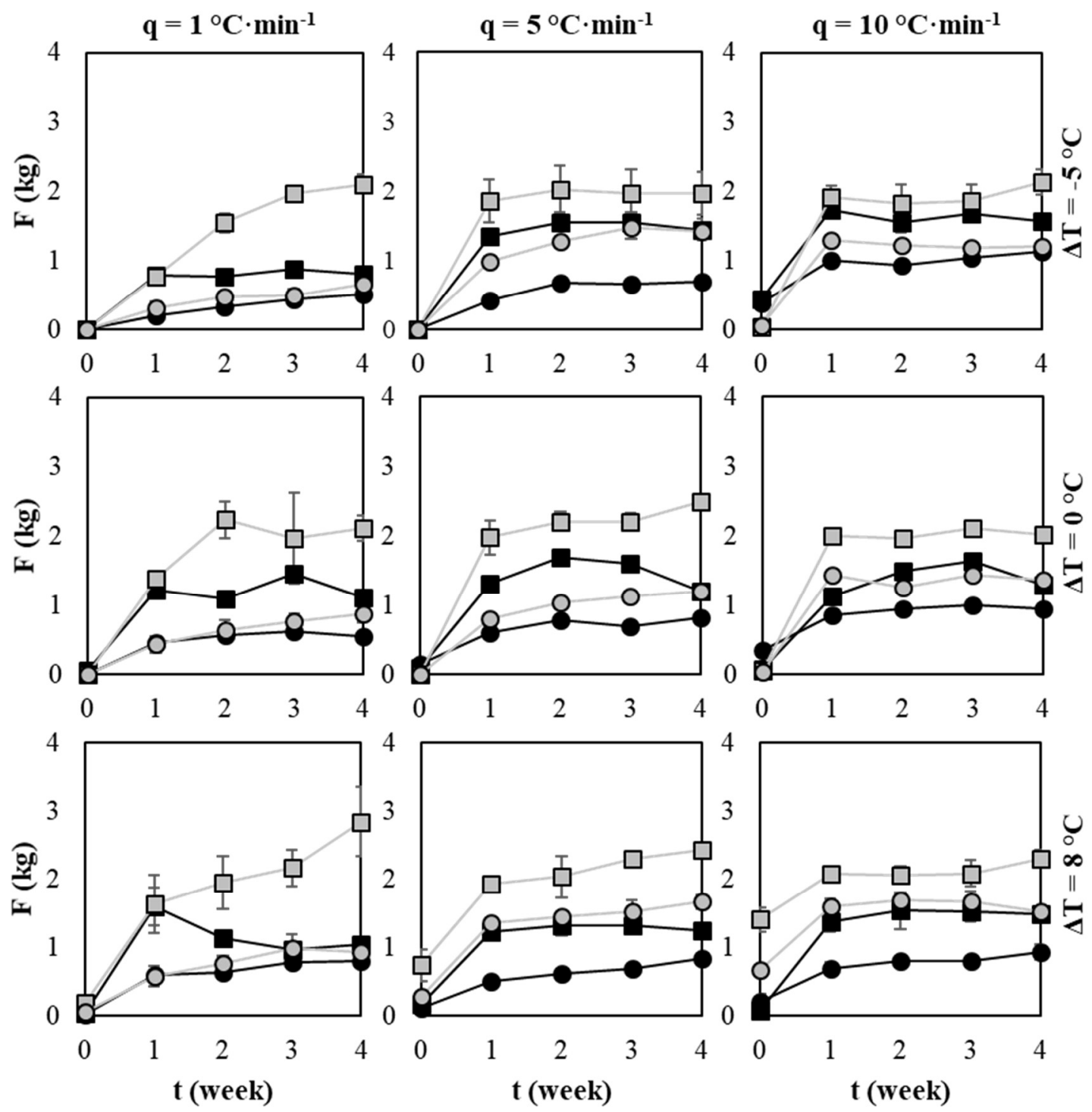

$\rightarrow \mathrm{PO}, \zeta=0 \rightarrow \mathrm{PMF}, \zeta=0 \quad \square-\mathrm{PO}, \zeta=1 \quad \mathrm{O}-\mathrm{PMF}, \zeta=1$

Figure 4.9 Effects of sugar and processing conditions on $F$ of $P O$ and PMF over time.

$$
\begin{aligned}
\hat{\mathrm{y}}=-0.0717 & +0.0226 q+0.0153 \Delta T+0.6576 t+0.0020 q^{2}-0.2443 t^{2} \\
& +0.0304 t^{3}-0.0027 q \Delta T+0.0039 \Delta T t-0.0006 q \Delta T t \\
& +\zeta\left(0.1320 t-0.1326 t^{2}-0.0002 q^{3}+0.0246 t^{3}\right. \\
& +0.1138 q t+0.0004 q^{2} \Delta T-0.0050 q^{2} t+0.0004 q \Delta T^{2} \\
& \left.-0.0136 q t^{2}\right)
\end{aligned}
$$

$\left(C_{P}=11.71, \mathrm{~F}_{18,251}=148.32, \mathrm{p}<0.0001\right)$. As observed with $\mathrm{G}^{\prime}$, the higher optimal cooling rate in PMF-sugar blends suggested an ability to undergo more rapid thermal processing in 
comparison to PO-sugar blends. Process maps of predicted F according to these models are illustrated in Figure 4.10.

Although PMF-sugar blends processed at $10{ }^{\circ} \mathrm{C} \cdot \mathrm{min}^{-1}$ to $28{ }^{\circ} \mathrm{C}$ exhibited final $\mathrm{G}^{\prime}$ values greater than their PO counterparts (Figure 4.4), their corresponding final $\mathrm{F}$ was lower (Figure 4.9). Dispersions can accelerate crystallization (e.g., carbon nanotubes in polypropylene) and enhance solid-like character (e.g., monoolein-stabilized emulsions) by reinforcing the continuous phase through direct interfacial interactions (Schawe et al., 2015; Rafanan and Rousseau, 2017). In that respect, it is then plausible that properties of fats such as $\mathrm{G}^{\prime}$ and $F$ will respond differently to the presence of a dispersed phase. For example, while a strong correlation between rheology and texture analysis existed in bulk oils $\left(\mathrm{R}^{2}=0.80, \rho=0.90\right)$, this was significantly weakened by sugar $\left(\mathrm{R}^{2}=0.69, \rho=0.83\right)$ (Figure 4.11). This was largely due to greater changes in rheology (i.e., small scale deformation) of oil-sugar blends in response to changes in processing conditions.

\subsubsection{Further Considerations with Multiple Regression}

The results of this study have shown that predicting how oil-sugar blends respond to processing from bulk oil data is not feasible, as further demonstrated from multiple regression analysis (Figure 4.12). The correlation between observed and predicted $\mathrm{G}^{\prime}$ were quite poor for PO-sugar $\left(\mathrm{R}^{2}=0.57, \rho=0.74\right)$ and PMF-sugar blends $\left(\mathrm{R}^{2}=0.43, \rho=0.66\right)$ where predictions were consistently lower than observed. Conversely, the multiple regression of the full dataset, which accounts for confectioner's sugar through the binary variable $\zeta$, significantly improved prediction for PO-sugar $\left(\mathrm{R}^{2}=0.84, \rho=0.91\right)$ and PMFsugar blends $\left(\mathrm{R}^{2}=0.86, \rho=0.93\right)$. This demonstrates the importance of $\zeta$, which corrects 

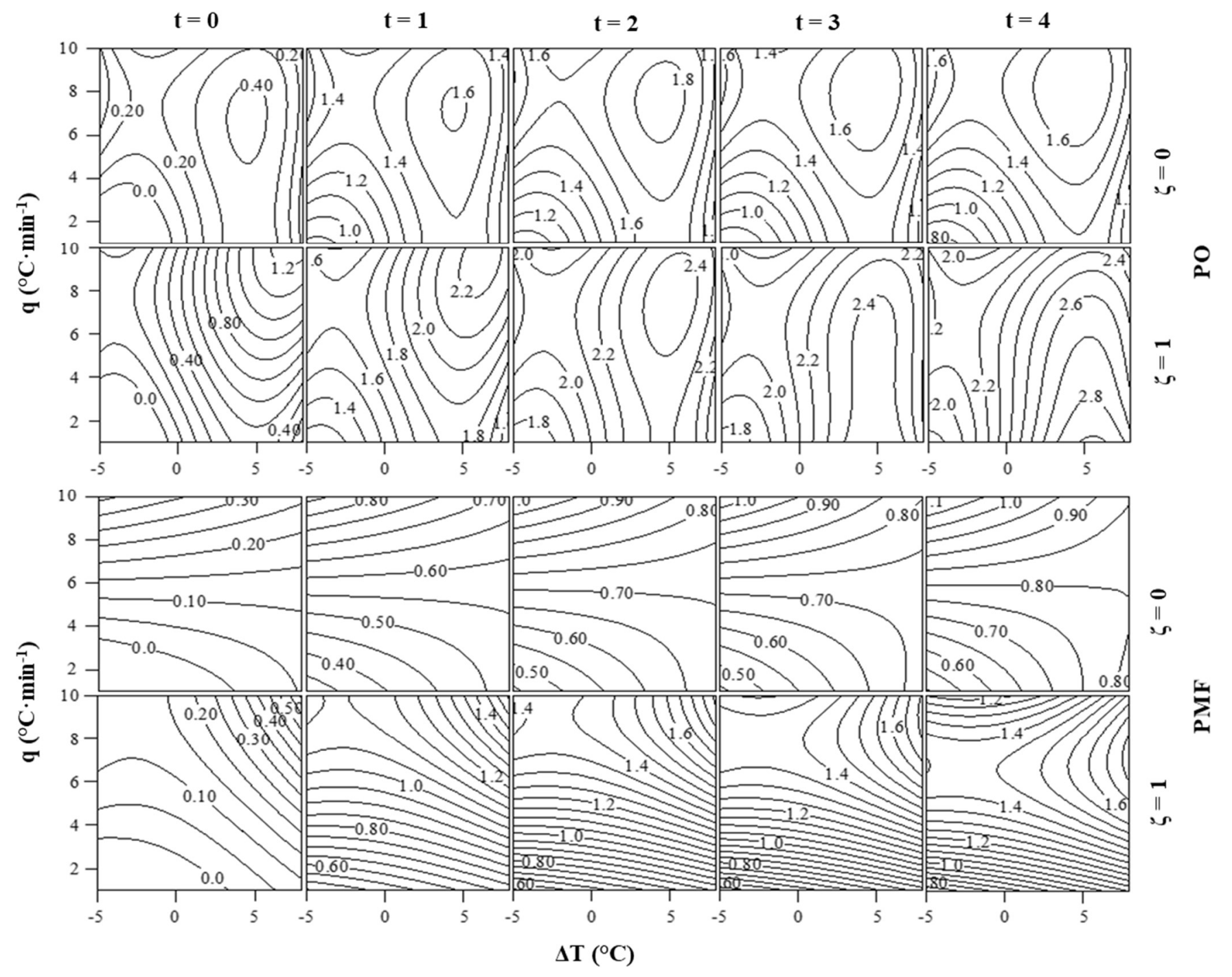

Figure 4.10 Predicted process maps of $F(\mathrm{~kg})$ according to models $4.3 a$ and $4.3 \mathrm{~b}$. 

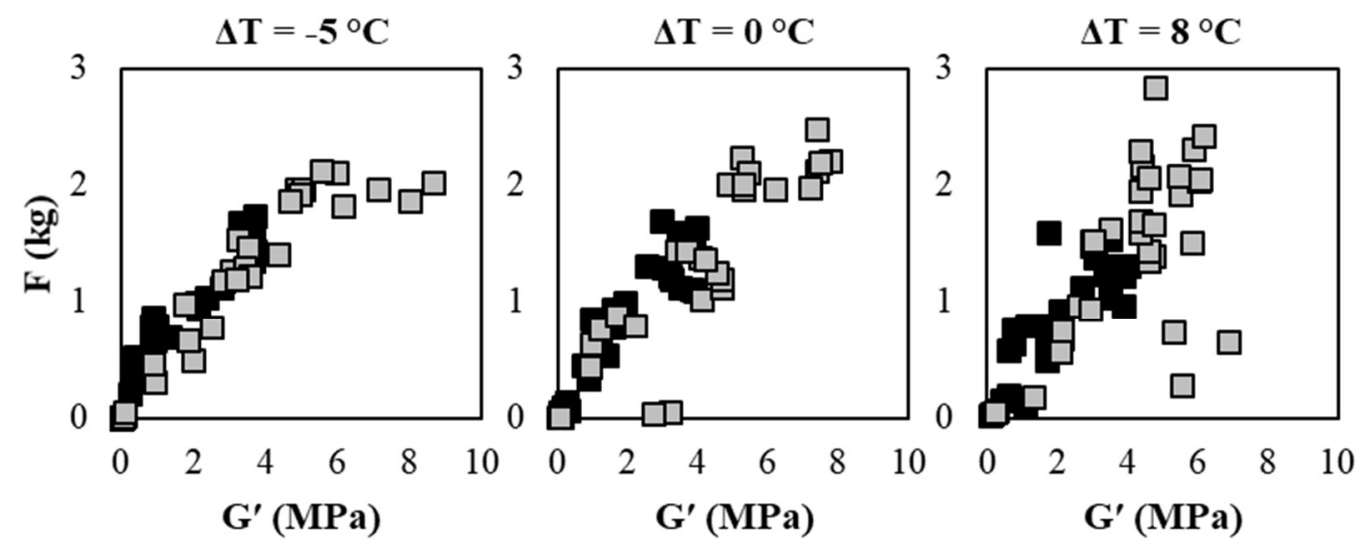

$\mathbf{\square} \zeta=0 \quad \square=1$

Figure 4.11 Effect of sugar and endpoint on the correlation between small and large deformation tests.
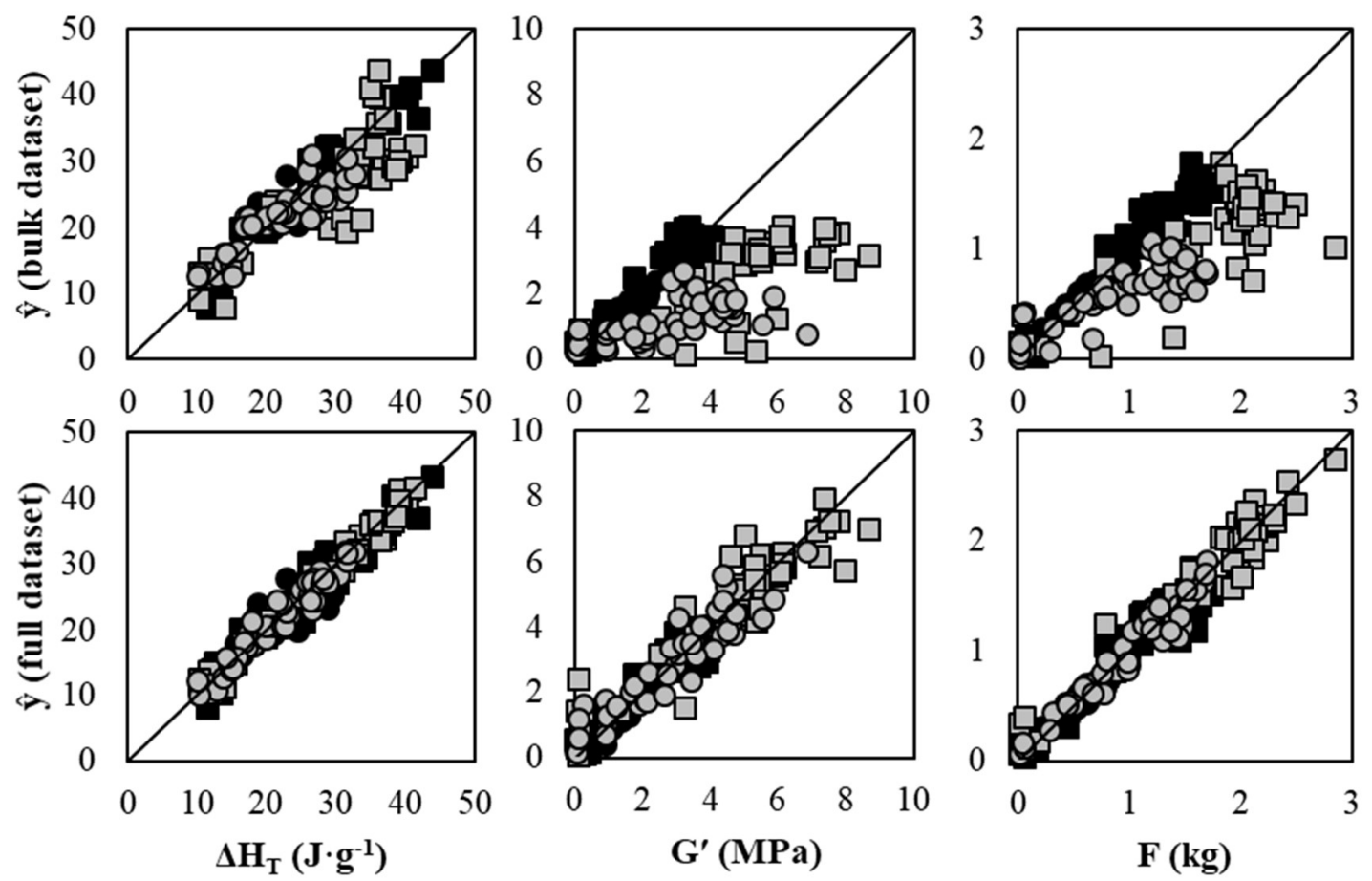

$\square \mathrm{PO}, \zeta=0 \quad \bullet \mathrm{PMF}, \zeta=0 \quad \square \mathrm{PO}, \zeta=1 \quad \mathrm{OPMF}, \zeta=1$

Figure 4.12 Comparison between observed and predicted responses from bulk (extrapolated) and full datasets. 
for the role of confectioner's sugar on the processing behaviour of bulk oils (e.g., fat crystal morphology, spatial distribution, reinforcement through interfacial interactions, etc.).

Multiple regression analysis also determined optimal process values of both bulk oils and oil-sugar blends (Table 4.1). It was evident from the observed data (Figures 4.1, 4.4, and 4.9) that these optimal process values would differ according to the type of oil. For example, the suppression of final $\mathrm{G}^{\prime}$ of bulk PO under rapid thermal processing produced an optimal cooling rate (i.e., $5.1{ }^{\circ} \mathrm{C} \cdot \mathrm{min}^{-1}$ ) lower than bulk PMF (i.e., $10.0{ }^{\circ} \mathrm{C} \cdot \mathrm{min}^{-1}$ ), where no suppression was observed. The addition of confectioner's sugar further confounded the interrelation between responses (Figure 4.11), particularly with more rapid thermal processing, which also resulted in optimal process values not only distinct from their bulk counterparts but also distinct for each response (Table 4.1).

The generation of these optimal process values not only gives insight towards processing requirements necessary for a maximum response, but also emphasizes the limitations of applying empirical knowledge from bulk studies to more complex systems. To further illustrate this, predicted responses of oil-sugar blends using their associated optimal processing values were compared against predicted responses using values optimized for their bulk counterparts (Figure 4.13). For example, applying optimal values for final $\mathrm{G}^{\prime}$ in bulk PMF (i.e., $q_{0}=10.0{ }^{\circ} \mathrm{C} \cdot \mathrm{min}^{-1}$ and $\Delta T_{0}=-5.0{ }^{\circ} \mathrm{C}$ ) towards PMF-sugar predicted a response 2.2 MPa lower than the actual maximum response had the proper conditions (i.e., $q_{0}=7.7^{\circ} \mathrm{C} \cdot \min ^{-1}$ and $\left.\Delta T_{0}=-2.1^{\circ} \mathrm{C}\right)$ been used. 

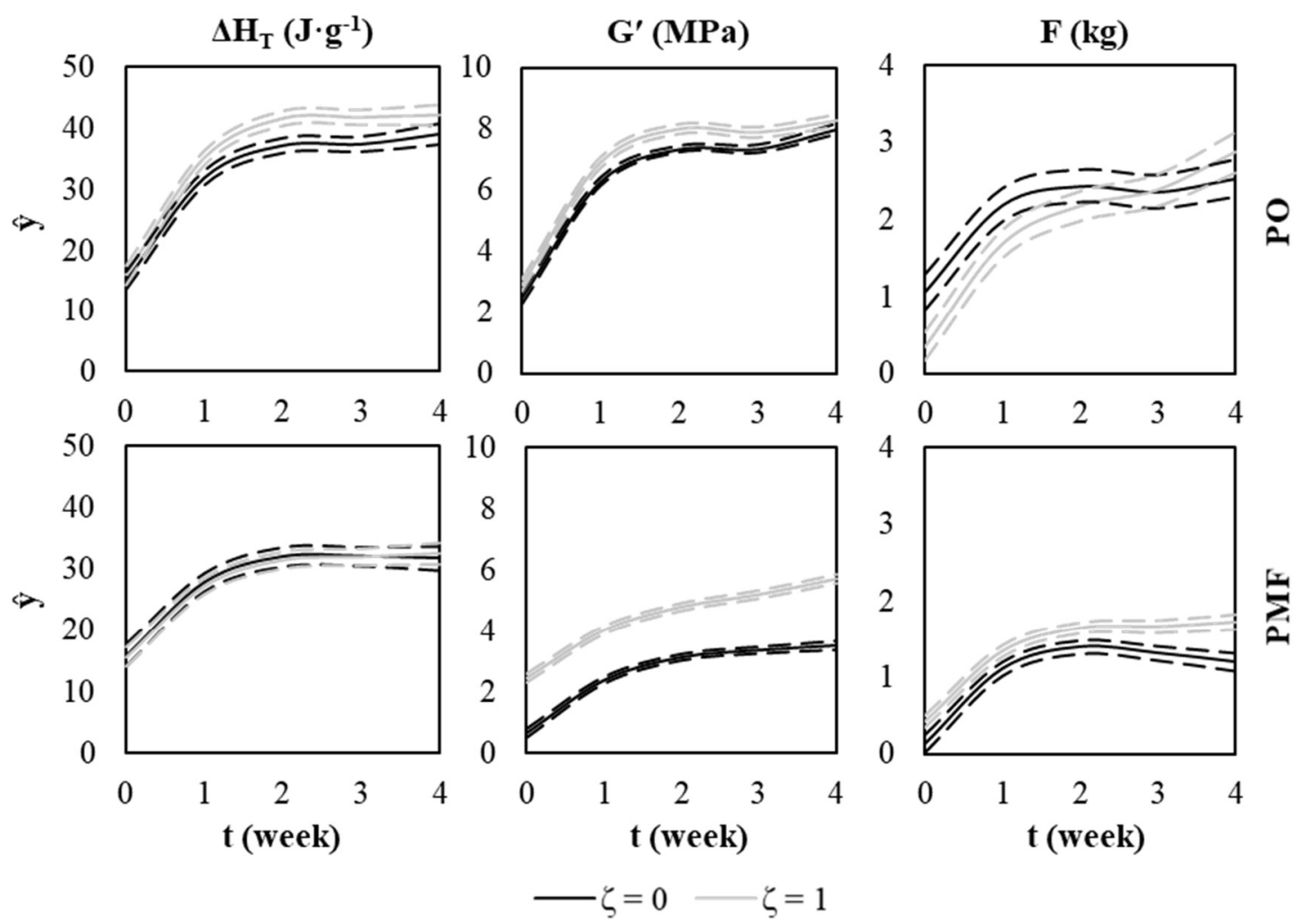

Figure 4.13 Comparison between maximum response of oil-sugar blends (95\% confidence limits, hashed line) against response predicted from process values optimized for bulk systems.

\subsection{Conclusions}

The response of palm-based confectionery systems to composition and processing during storage was examined. The addition of confectioner's sugar, which generally enhanced fat crystallization under most processing conditions, reinforced the fat crystalline network and may have facilitated interfacial interactions to increase $\mathrm{G}^{\prime}$ and $\mathrm{F}$ in these systems. Initial physical properties were particularly sensitive to processing in the presence of sugar, however, differences between processing conditions were minimized throughout storage. Subset models that account for the overall impact of confectioner's sugar on processing 
responses by using the binary variable $\zeta$ and identify optimal processing conditions were generated to overcome such issues and any extrapolative limitations from bulk systems. 


\section{Chapter 5:}

\section{GENERAL CONCLUSIONS AND FUTURE WORK}

The effect of confectioner's sugar on processing behavior in PO and PMF was explored in this dissertation. In general, fat network rigidity was enhanced by sugar, increasing elasticity and firmness. Not only did its addition obfuscate the interrelationship between these properties, but the oil-sugar blends exhibited greater sensitivity towards processing in comparison to the bulk counterparts questioning the ability of applying trends from bulk oils towards multi-ingredient systems. Furthermore, confectioner's sugar appeared to suppress $\beta$ crystals under most processing conditions. Subset models that account for the overall impact of confectioner's sugar through binary variable $\zeta$ were established to overcome limitations associated with extrapolation. These models were also used to create process maps and identify optimal processing conditions to yield maximum response. The technique of multiple regression used herein may be utilized as a novel industrial tool towards production efficiency and final quality.

Given the importance of this binary variable, it would be of great benefit to design additional experiments that begin its deconvolution. While confectioner's sugar is generally considered to behave as a catalytic impurity that aids in heterogeneous nucleation, neither brightfield microscopy nor CLSM within this dissertation could conclude this. Freeze-fracturing these oil-sugar blends followed by the interfacial characterization through cryogenic scanning electron microscopy may be used to possibly identify fat crystals localized at the interface. This technique has nanoscale resolution which may reveal details regarding interfacial crystallization that other methods cannot achieve. Furthermore, the use of force 
spectroscopy to understand specific molecular interactions between the phases may provide novel insight towards interfacial characterization.

Another consideration for future work is the characterization of tortuosity within these oilsugar blends and how this impacts fat crystallization. Diffusion coefficients of liquid-state TAGs measured by pulsed gradient stimulated echo magnetic resonance can be used to characterize structure and calculate tortuosity. This could be coupled with images obtained from rheo-NMR experiments to calculate velocity distributions and phase transitions. The information gained from such experiments may allow for a better understanding of the mechanisms behind the slow crystallization in PO and how the presence of a dispersed ingredients further affects this.

Additional considerations for future work include other rheological analyses to better understand how the addition of confectioner's sugar impacts the physical properties of PO and PMF (e.g., creep-recovery test, thixotropy test), cross-validating the predictive subset models by other means to correct for overfitting (e.g., leave-one-out cross-validation), collecting dimensional parameters of fat crystals via CLSM for further regression analysis, and finally comparing and contrasting these results with a pilot plant scaleup for the continued interests of the food industry. 


\section{Appendices}

\section{A1 Frequency Sweep Tests}
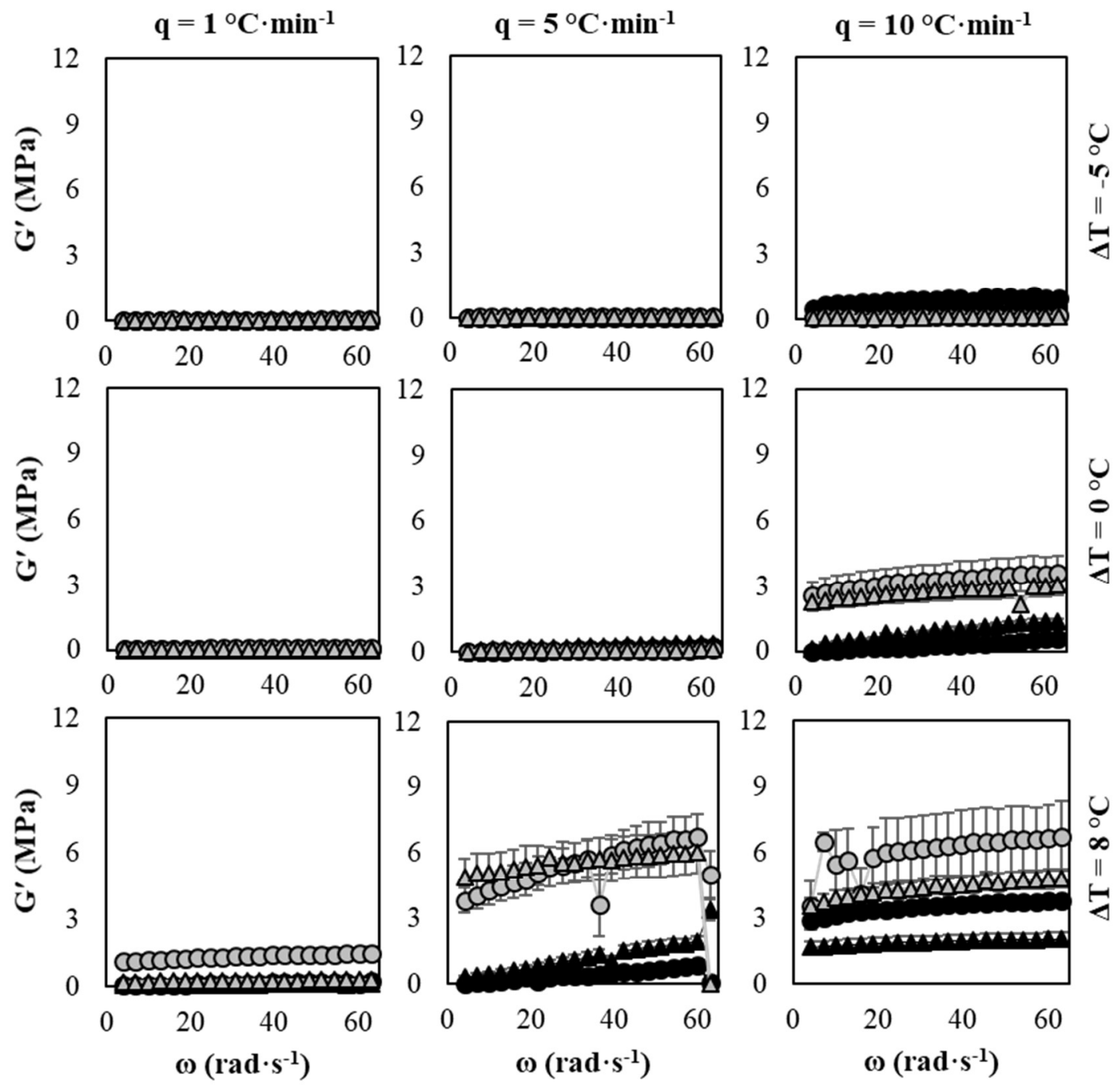

$\rightarrow \mathrm{PO}, \zeta=0 \rightarrow \mathrm{PMF}, \zeta=0 \quad \mathrm{O}-\mathrm{PO}, \zeta=1 \quad \Delta \mathrm{PMF}, \zeta=1$

Figure A1.1 Effects of sugar and processing conditions on $G^{\prime}$ of $P O$ and PMF over angular frequency ( $t=0$ weeks). 

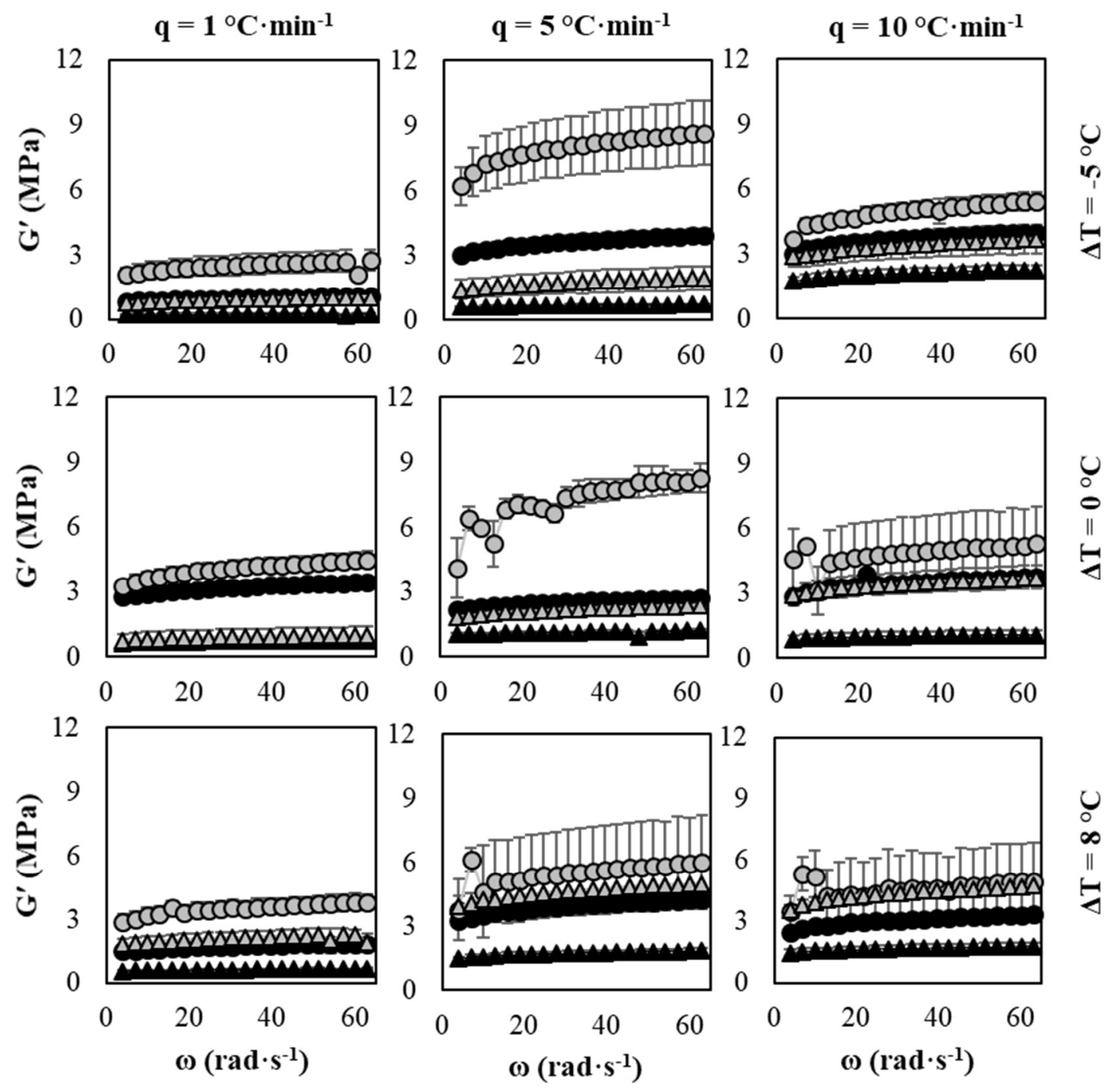

$\rightarrow \mathrm{PO}, \zeta=0 \rightarrow \mathrm{PMF}, \zeta=0 \quad \mathrm{O}-\mathrm{PO}, \zeta=1 \quad \Delta \mathrm{PMF}, \zeta=1$

Figure A1.2 Effects of sugar and processing conditions on $G^{\prime}$ of PO and PMF over angular frequency $(t=1$ week $)$. 

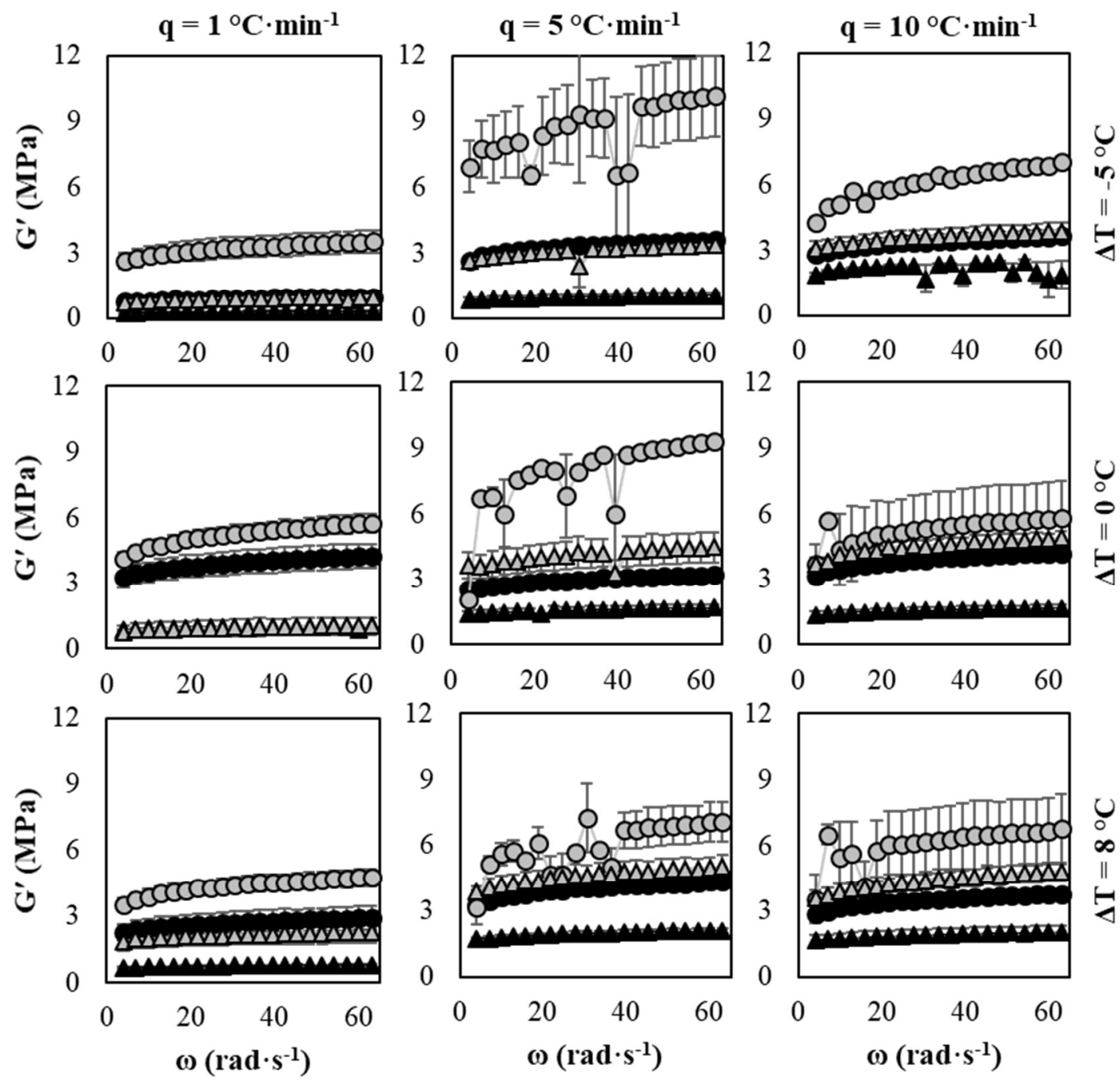

$\rightarrow \mathrm{PO}, \zeta=0 \rightarrow \mathrm{PMF}, \zeta=0 \quad \mathrm{O}-\mathrm{PO}, \zeta=1 \quad \Delta \mathrm{PMF}, \zeta=1$

Figure A1.3 Effects of sugar and processing conditions on $G^{\prime}$ of $P O$ and PMF over angular frequency ( $t=2$ weeks). 

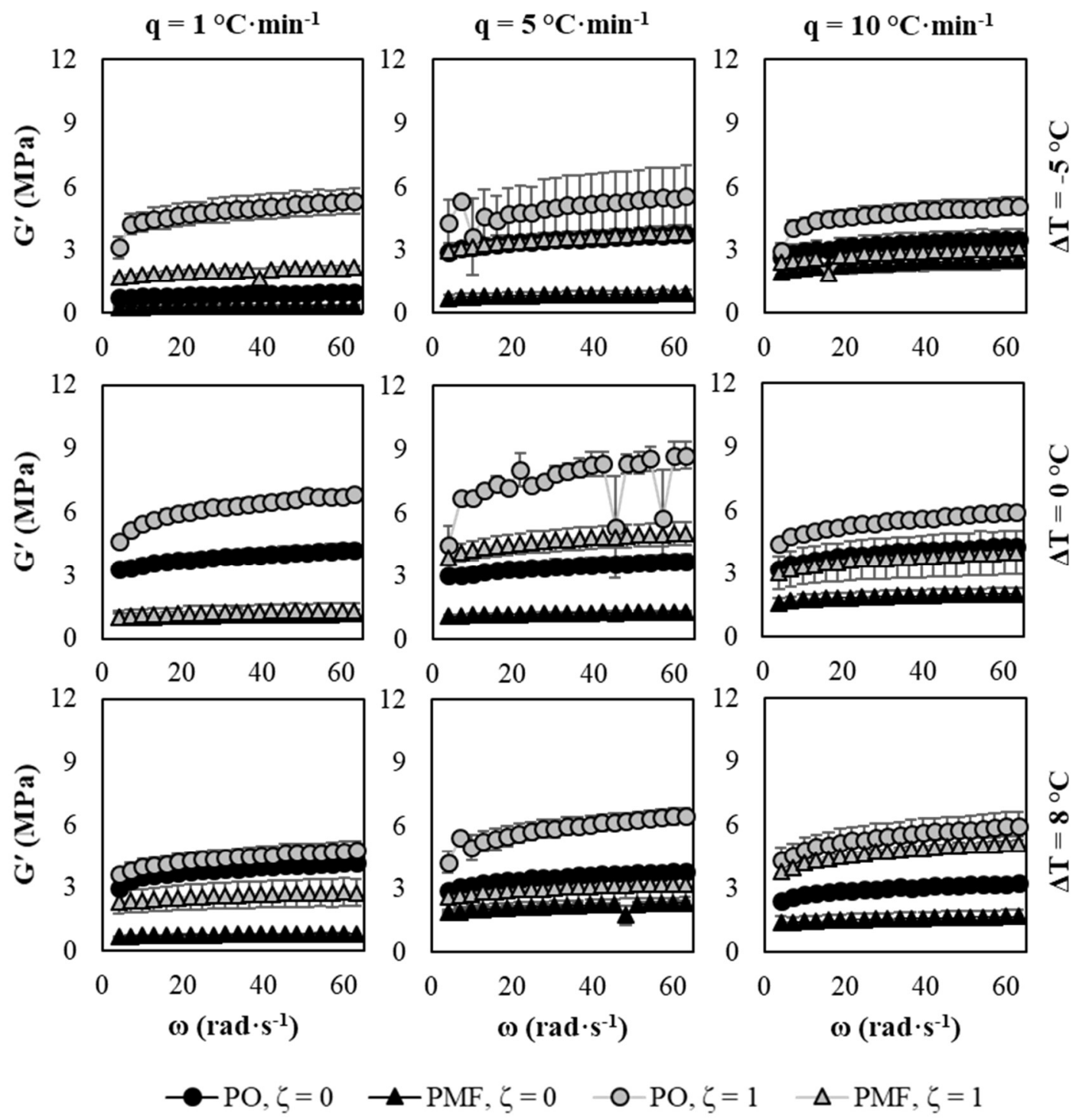

Figure A1.4 Effects of sugar and processing conditions on $G^{\prime}$ of $P O$ and PMF over angular frequency $(t=3$ weeks). 

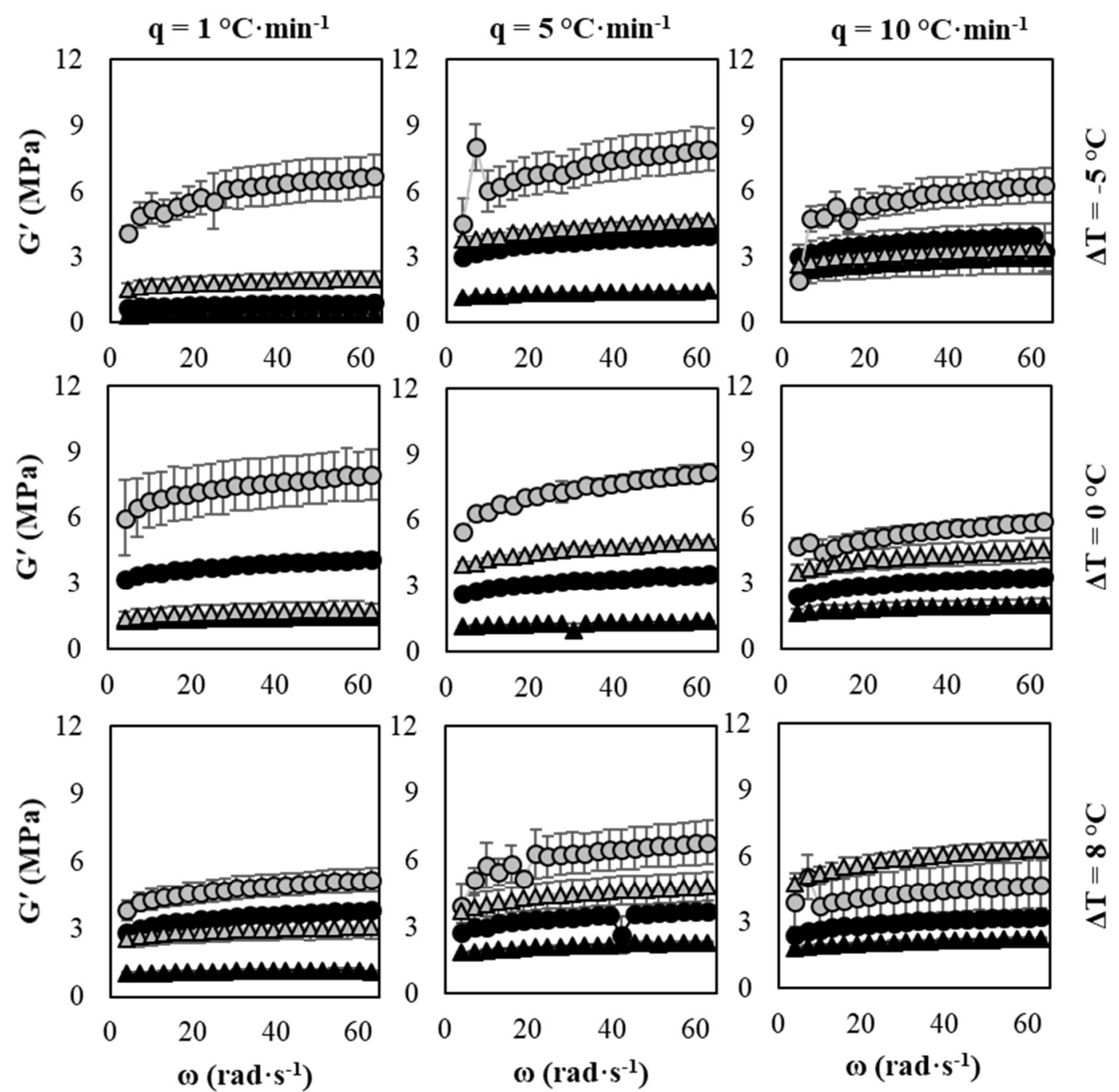

$\rightarrow \mathrm{PO}, \zeta=0 \quad \neg$ PMF, $\zeta=0 \quad-\mathrm{OPO}, \zeta=1 \quad \Delta-\mathrm{PMF}, \zeta=1$

Figure A1.5 Effects of sugar and processing conditions on $G^{\prime}$ of $P O$ and PMF over angular frequency ( $t=4$ weeks). 


\section{A2 ANOVA Tables}

\section{A2.1 Chapter 2}

Table A2.1.1 ANOVA for SFC. ${ }^{A 2.1 .1}$

\begin{tabular}{|c|c|c|c|c|c|c|}
\hline Source & & DF & SS & MS & $\mathbf{F}$ & $\mathbf{p}$ \\
\hline \multicolumn{7}{|l|}{ Model } \\
\hline & Replicate & 2 & $3.57 \mathrm{E}+01$ & $1.79 \mathrm{E}+01$ & 11.95 & $<0.0001$ \\
\hline & oil & 1 & $5.10 \mathrm{E}+03$ & $5.10 \mathrm{E}+03$ & 3409.89 & $<0.0001$ \\
\hline & oil $\times$ sugar & 1 & $1.68 \mathrm{E}+02$ & $1.68 \mathrm{E}+02$ & 112.29 & $<0.0001$ \\
\hline & $o i l \times t$ & 4 & $4.86 \mathrm{E}+02$ & $1.22 \mathrm{E}+02$ & 81.34 & $<0.0001$ \\
\hline & oil $\times$ sugar $\times t$ & 4 & $3.21 \mathrm{E}+02$ & $8.02 \mathrm{E}+01$ & 53.68 & $<0.0001$ \\
\hline & sugar & 1 & $1.10 \mathrm{E}+03$ & $1.10 \mathrm{E}+03$ & 739.21 & $<0.0001$ \\
\hline & sugar $\times t$ & 4 & $2.85 \mathrm{E}+02$ & $7.14 \mathrm{E}+01$ & 47.75 & $<0.0001$ \\
\hline & $t$ & 4 & $2.30 \mathrm{E}+03$ & $5.76 \mathrm{E}+02$ & 385.45 & $<0.0001$ \\
\hline Error & & 338 & $5.05 \mathrm{E}+02$ & $1.49 \mathrm{E}+00$ & & \\
\hline Total & & 359 & $1.03 \mathrm{E}+04$ & & & \\
\hline
\end{tabular}

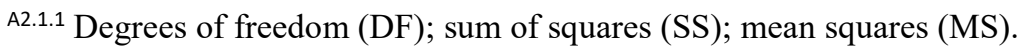


Table A2.1.2 ANOVA for $\triangle H_{T}{ }^{A 2.1 .2}$

\begin{tabular}{ccccccc}
\hline Source & & DF & SS & MS & F & p \\
\hline Model & & & & & & \\
& oil & 1 & $1.59 \mathrm{E}+02$ & $1.59 \mathrm{E}+02$ & 127.10 & $<0.0001$ \\
& oil $\times$ sugar & 1 & $6.61 \mathrm{E}+01$ & $6.61 \mathrm{E}+01$ & 53.00 & $<0.0001$ \\
& oil $\times$ t & 4 & $6.24 \mathrm{E}+01$ & $1.56 \mathrm{E}+01$ & 12.51 & $<0.0001$ \\
& sugar & 1 & $1.67 \mathrm{E}+01$ & $1.67 \mathrm{E}+01$ & 13.35 & 0.0006 \\
& $t$ & 4 & $1.07 \mathrm{E}+03$ & $2.67 \mathrm{E}+02$ & 213.60 & $<0.0001$ \\
Error & & 48 & $5.99 \mathrm{E}+01$ & $1.25 \mathrm{E}+00$ & & \\
Total & & 59 & $1.43 \mathrm{E}+03$ & & & \\
\hline
\end{tabular}

A2.1.2 Degrees of freedom (DF); sum of squares (SS); mean squares (MS). 
Table A2.1.3 ANOVA for $G^{\prime}{ }^{A 2.1 .3}$

\begin{tabular}{|c|c|c|c|c|c|c|}
\hline Source & & DF & SS & MS & $\mathbf{F}$ & p \\
\hline \multicolumn{7}{|l|}{ Model } \\
\hline & Replicate & 2 & $2.14 \mathrm{E}+01$ & $1.07 \mathrm{E}+01$ & 34.91 & $<0.0001$ \\
\hline & $\omega$ & 20 & $3.58 \mathrm{E}+01$ & $1.79 \mathrm{E}+00$ & 5.84 & $<0.0001$ \\
\hline & oil & 1 & $2.61 \mathrm{E}+03$ & $2.61 \mathrm{E}+03$ & 8506.29 & $<0.0001$ \\
\hline & oil $\times$ sugar & 1 & $1.77 \mathrm{E}+02$ & $1.77 \mathrm{E}+02$ & 577.55 & $<0.0001$ \\
\hline & $o i l \times t$ & 4 & $6.83 \mathrm{E}+02$ & $1.71 \mathrm{E}+02$ & 556.73 & $<0.0001$ \\
\hline & oil $\times$ sugar $\times t$ & 4 & $1.03 \mathrm{E}+02$ & $2.58 \mathrm{E}+01$ & 84.21 & $<0.0001$ \\
\hline & sugar & 1 & $2.38 \mathrm{E}+02$ & $2.38 \mathrm{E}+02$ & 776.10 & $<0.0001$ \\
\hline & sugar $\times t$ & 4 & $1.30 \mathrm{E}+02$ & $3.26 \mathrm{E}+01$ & 106.22 & $<0.0001$ \\
\hline & $t$ & 4 & $1.86 \mathrm{E}+03$ & $4.65 \mathrm{E}+02$ & 1517.05 & $<0.0001$ \\
\hline Error & & 1212 & $3.72 \mathrm{E}+02$ & $3.07 \mathrm{E}-01$ & & \\
\hline Total & & 1253 & $6.26 \mathrm{E}+03$ & & & \\
\hline
\end{tabular}

A2.1.3 Degrees of freedom (DF); sum of squares (SS); mean squares (MS). 
Table A2.1.4 ANOVA for $G^{\prime \prime}{ }^{\text {. A2.1.4 }}$

\begin{tabular}{|c|c|c|c|c|c|c|}
\hline Source & & DF & SS & MS & $\mathbf{F}$ & $\mathbf{p}$ \\
\hline \multicolumn{7}{|l|}{ Model } \\
\hline & Replicate & 2 & $9.20 \mathrm{E}-02$ & $4.60 \mathrm{E}-02$ & 6.51 & 0.0015 \\
\hline & $\omega$ & 20 & $1.10 \mathrm{E}+00$ & $5.48 \mathrm{E}-02$ & 7.75 & $<0.0001$ \\
\hline & oil & 1 & $3.75 \mathrm{E}+01$ & $3.75 \mathrm{E}+01$ & 5310.74 & $<0.0001$ \\
\hline & oil $\times$ sugar & 1 & $1.89 \mathrm{E}+00$ & $1.89 \mathrm{E}+00$ & 267.65 & $<0.0001$ \\
\hline & $o i l \times t$ & 4 & $9.77 \mathrm{E}+00$ & $2.44 \mathrm{E}+00$ & 345.64 & $<0.0001$ \\
\hline & oil $\times$ sugar $\times t$ & 4 & $9.00 \mathrm{E}-01$ & $2.25 \mathrm{E}-01$ & 31.83 & $<0.0001$ \\
\hline & sugar & 1 & $3.56 \mathrm{E}+00$ & $3.56 \mathrm{E}+00$ & 502.87 & $<0.0001$ \\
\hline & sugar $\times t$ & 4 & $1.40 \mathrm{E}+00$ & $3.50 \mathrm{E}-01$ & 49.58 & $<0.0001$ \\
\hline & $t$ & 4 & $2.22 \mathrm{E}+01$ & $5.54 \mathrm{E}+00$ & 783.68 & $<0.0001$ \\
\hline Error & & 1212 & $8.57 \mathrm{E}+00$ & 7.07E-03 & & \\
\hline Total & & 1253 & $8.74 \mathrm{E}+01$ & & & \\
\hline
\end{tabular}

A2.1.4 Degrees of freedom (DF); sum of squares (SS); mean squares (MS). 


\section{A2.2 Chapter 3}

Table A2.2.1 ANOVA for SFC. ${ }^{A 2.2 .1}$

\begin{tabular}{ccccccc}
\hline Source & & DF & SS & MS & F & p \\
\hline Model & & & & & & \\
& Replicate & 2 & $2.66 \mathrm{E}+01$ & $1.33 \mathrm{E}+01$ & 8.42 & 0.0002 \\
oil & 1 & $7.11 \mathrm{E}+03$ & $7.11 \mathrm{E}+03$ & 4508.63 & $<0.0001$ \\
& oil $\times \zeta$ & 1 & $2.16 \mathrm{E}+02$ & $2.16 \mathrm{E}+02$ & 137.10 & $<0.0001$ \\
oil $\times \Delta T$ & 2 & $8.59 \mathrm{E}+02$ & $4.29 \mathrm{E}+02$ & 272.21 & $<0.0001$ \\
& oil $\times t$ & 4 & $7.07 \mathrm{E}+02$ & $1.77 \mathrm{E}+02$ & 112.11 & $<0.0001$ \\
& oil $\times \zeta \times \Delta T$ & 2 & $4.58 \mathrm{E}+02$ & $2.29 \mathrm{E}+02$ & 145.33 & $<0.0001$ \\
& oil $\times \zeta \times t$ & 4 & $1.93 \mathrm{E}+02$ & $4.83 \mathrm{E}+01$ & 30.61 & $<0.0001$ \\
& oil $\times \Delta T \times t$ & 8 & $1.76 \mathrm{E}+02$ & $2.20 \mathrm{E}+01$ & 13.94 & $<0.0001$ \\
& oil $\times \zeta \times \Delta T \times t$ & 8 & $1.80 \mathrm{E}+02$ & $2.25 \mathrm{E}+01$ & 14.24 & $<0.0001$ \\
& $\zeta$ & 1 & $6.79 \mathrm{E}+02$ & $6.79 \mathrm{E}+02$ & 430.68 & $<0.0001$ \\
& $\zeta \times \Delta T$ & 2 & $6.90 \mathrm{E}+03$ & $3.45 \mathrm{E}+03$ & 2188.46 & $<0.0001$ \\
& $\zeta \times t$ & 4 & $2.19 \mathrm{E}+02$ & $5.47 \mathrm{E}+01$ & 34.68 & $<0.0001$ \\
& $\zeta \times \Delta T \times t$ & 8 & $6.22 \mathrm{E}+02$ & $7.77 \mathrm{E}+01$ & 49.29 & $<0.0001$ \\
& $\Delta T$ & 2 & $5.11 \mathrm{E}+03$ & $2.55 \mathrm{E}+03$ & 1618.51 & $<0.0001$ \\
& $\Delta T \times t$ & 8 & $5.55 \mathrm{E}+02$ & $6.93 \mathrm{E}+01$ & 43.95 & $<0.0001$ \\
& $t$ & 4 & $2.91 \mathrm{E}+03$ & $7.27 \mathrm{E}+02$ & 461.05 & $<0.0001$ \\
& 1018 & $1.61 \mathrm{E}+03$ & $1.58 \mathrm{E}+00$ & & \\
Error & 1079 & $2.85 \mathrm{E}+04$ & & & \\
& & & & & &
\end{tabular}

A2.2.1 Degrees of freedom (DF); sum of squares (SS); mean squares (MS). 
Table A2.2.2 ANOVA for $G^{\prime}{ }^{\prime 2.2 .2}$

\begin{tabular}{|c|c|c|c|c|c|c|}
\hline Source & & DF & SS & MS & $\mathbf{F}$ & $\mathbf{p}$ \\
\hline \multicolumn{7}{|l|}{ Model } \\
\hline & Replicate & 2 & $2.55 \mathrm{E}+01$ & $1.28 \mathrm{E}+01$ & 53.47 & $<0.0001$ \\
\hline & $\omega$ & 20 & $7.69 \mathrm{E}+01$ & $3.85 \mathrm{E}+00$ & 16.10 & $<0.0001$ \\
\hline & oil & 1 & $3.65 \mathrm{E}+03$ & $3.65 E+03$ & 15272.90 & $<0.0001$ \\
\hline & $o i l \times \zeta$ & 1 & $2.60 \mathrm{E}+02$ & $2.60 \mathrm{E}+02$ & 1089.95 & $<0.0001$ \\
\hline & $o i l \times \Delta T$ & 2 & $4.17 \mathrm{E}+02$ & $2.09 \mathrm{E}+02$ & 873.65 & $<0.0001$ \\
\hline & $o i l \times t$ & 4 & $8.58 \mathrm{E}+02$ & $2.14 \mathrm{E}+02$ & 897.52 & $<0.0001$ \\
\hline & $o i l \times \zeta \times \Delta T$ & 2 & $1.41 \mathrm{E}+02$ & $7.04 \mathrm{E}+01$ & 294.49 & $<0.0001$ \\
\hline & $o i l \times \zeta \times t$ & 4 & $8.82 \mathrm{E}+01$ & $2.20 \mathrm{E}+01$ & 92.25 & $<0.0001$ \\
\hline & oil $\times \Delta T \times t$ & 8 & $1.55 \mathrm{E}+02$ & $1.94 \mathrm{E}+01$ & 81.33 & $<0.0001$ \\
\hline & oil $\times \zeta \times \Delta T \times t$ & 8 & $1.90 \mathrm{E}+02$ & $2.38 \mathrm{E}+01$ & 99.44 & $<0.0001$ \\
\hline & $\zeta$ & 1 & $1.61 \mathrm{E}+03$ & $1.61 \mathrm{E}+03$ & 6751.31 & $<0.0001$ \\
\hline & $\zeta \times \Delta T$ & 2 & $1.19 \mathrm{E}+02$ & $5.96 \mathrm{E}+01$ & 249.67 & $<0.0001$ \\
\hline & $\zeta \times t$ & 4 & $4.24 \mathrm{E}+02$ & $1.06 \mathrm{E}+02$ & 444.12 & $<0.0001$ \\
\hline & $\zeta \times \Delta T \times t$ & 8 & $1.82 \mathrm{E}+02$ & $2.27 \mathrm{E}+01$ & 95.03 & $<0.0001$ \\
\hline & $\Delta T$ & 2 & $7.03 \mathrm{E}+02$ & $3.52 \mathrm{E}+02$ & 1471.65 & $<0.0001$ \\
\hline & $\Delta T \times t$ & 8 & $1.64 \mathrm{E}+02$ & $2.05 \mathrm{E}+01$ & 85.95 & $<0.0001$ \\
\hline & $t$ & 4 & $3.61 \mathrm{E}+03$ & $9.01 \mathrm{E}+02$ & 3772.65 & $<0.0001$ \\
\hline Error & & 3682 & $8.80 \mathrm{E}+02$ & $2.39 \mathrm{E}-01$ & & \\
\hline Total & & 3763 & $1.36 \mathrm{E}+04$ & & & \\
\hline
\end{tabular}

A2.2.2 Degrees of freedom (DF); sum of squares (SS); mean squares (MS). 
Table A2.2.3 ANOVA for $G^{\prime \prime}{ }^{.22 .2 .3}$

\begin{tabular}{|c|c|c|c|c|c|c|}
\hline Source & & DF & SS & MS & $\mathbf{F}$ & p \\
\hline \multicolumn{7}{|l|}{ Model } \\
\hline & Replicate & 2 & $2.14 \mathrm{E}-01$ & 1.07E-01 & 9.77 & $<0.0001$ \\
\hline & $\omega$ & 20 & $1.91 \mathrm{E}+00$ & 9.54E-02 & 8.71 & $<0.0001$ \\
\hline & oil & 1 & $5.24 \mathrm{E}+01$ & $5.24 \mathrm{E}+01$ & 4784.17 & $<0.0001$ \\
\hline & $o i l \times \zeta$ & 1 & $2.77 \mathrm{E}+00$ & $2.77 \mathrm{E}+00$ & 253.31 & $<0.0001$ \\
\hline & $o i l \times \Delta T$ & 2 & $6.04 \mathrm{E}+00$ & $3.02 \mathrm{E}+00$ & 275.99 & $<0.0001$ \\
\hline & $o i l \times t$ & 4 & $1.37 \mathrm{E}+01$ & $3.43 \mathrm{E}+00$ & 313.27 & $<0.0001$ \\
\hline & $o i l \times \zeta \times \Delta T$ & 2 & $1.95 \mathrm{E}+00$ & $9.75 \mathrm{E}-01$ & 89.05 & $<0.0001$ \\
\hline & $o i l \times \zeta \times t$ & 4 & $1.06 \mathrm{E}+00$ & $2.65 \mathrm{E}-01$ & 24.15 & $<0.0001$ \\
\hline & oil $\times \Delta T \times t$ & 8 & $2.51 \mathrm{E}+00$ & $3.14 \mathrm{E}-01$ & 28.63 & $<0.0001$ \\
\hline & oil $\times \zeta \times \Delta T \times t$ & 8 & $2.88 \mathrm{E}+00$ & $3.60 \mathrm{E}-01$ & 32.90 & $<0.0001$ \\
\hline & $\zeta$ & 1 & $1.76 \mathrm{E}+01$ & $1.76 \mathrm{E}+01$ & 1607.79 & $<0.0001$ \\
\hline & $\zeta \times \Delta T$ & 2 & $1.84 \mathrm{E}+00$ & $9.21 \mathrm{E}-01$ & 84.12 & $<0.0001$ \\
\hline & $\zeta \times t$ & 4 & $4.25 \mathrm{E}+00$ & $1.06 \mathrm{E}+00$ & 96.96 & $<0.0001$ \\
\hline & $\zeta \times \Delta T \times t$ & 8 & $3.69 \mathrm{E}+00$ & $4.61 \mathrm{E}-01$ & 42.08 & $<0.0001$ \\
\hline & $\Delta T$ & 2 & $6.96 \mathrm{E}+00$ & $3.48 \mathrm{E}+00$ & 317.83 & $<0.0001$ \\
\hline & $\Delta T \times t$ & 8 & $2.30 \mathrm{E}+00$ & $2.88 \mathrm{E}-01$ & 26.27 & $<0.0001$ \\
\hline & $t$ & 4 & $4.11 \mathrm{E}+01$ & $1.03 \mathrm{E}+01$ & 938.03 & $<0.0001$ \\
\hline Error & & 3682 & $4.03 \mathrm{E}+01$ & $1.10 \mathrm{E}-02$ & & \\
\hline Total & & 3763 & $2.04 \mathrm{E}+02$ & & & \\
\hline
\end{tabular}

A2.2.3 Degrees of freedom (DF); sum of squares (SS); mean squares (MS). 
Table A2.2.4 ANOVA for $F .^{A 2.2 .4}$

\begin{tabular}{ccccccc}
\hline Source & & DF & SS & MS & F & p \\
\hline Model & & & & & & \\
& Replicate & 2 & $8.60 \mathrm{E}+05$ & $4.30 \mathrm{E}+05$ & 5.62 & 0.0044 \\
& oil & 1 & $2.27 \mathrm{E}+07$ & $2.27 \mathrm{E}+07$ & 297.14 & $<0.0001$ \\
& oil $\times t$ & 4 & $5.29 \mathrm{E}+06$ & $1.32 \mathrm{E}+06$ & 17.27 & $<0.0001$ \\
& oil $\times \zeta \times t$ & 5 & $5.94 \mathrm{E}+06$ & $1.19 \mathrm{E}+06$ & 15.51 & $<0.0001$ \\
& $\zeta$ & 1 & $6.51 \mathrm{E}+06$ & $6.51 \mathrm{E}+06$ & 85.11 & $<0.0001$ \\
& $\zeta \times t$ & 4 & $4.08 \mathrm{E}+06$ & $1.02 \mathrm{E}+06$ & 13.31 & $<0.0001$ \\
& $\Delta T$ & 2 & $3.36 \mathrm{E}+06$ & $1.68 \mathrm{E}+06$ & 21.96 & $<0.0001$ \\
& $t$ & 4 & $3.20 \mathrm{E}+07$ & $8.00 \mathrm{E}+06$ & 104.53 & $<0.0001$ \\
Error & & 156 & $1.19 \mathrm{E}+07$ & $7.65 \mathrm{E}+04$ & & \\
\hline
\end{tabular}

A2.2.4 Degrees of freedom (DF); sum of squares (SS); mean squares (MS). 


\section{A2.3 Chapter 4}

Table A2.3.1 ANOVA for $\triangle H_{T .}{ }^{A 2.3 .1}$

\begin{tabular}{|c|c|c|c|c|c|c|}
\hline Source & & DF & SS & MS & $\mathbf{F}$ & p \\
\hline \multicolumn{7}{|l|}{ Model } \\
\hline & Replicate & 2 & $3.08 \mathrm{E}+01$ & $1.54 \mathrm{E}+01$ & 5.44 & 0.0047 \\
\hline & oil & 1 & $3.55 \mathrm{E}+03$ & $3.55 \mathrm{E}+03$ & 1256.56 & $<0.0001$ \\
\hline & oil $\times \zeta$ & 1 & $2.43 \mathrm{E}+02$ & $2.43 \mathrm{E}+02$ & 85.82 & $<0.0001$ \\
\hline & $o i l \times q$ & 2 & $4.68 \mathrm{E}+02$ & $2.34 \mathrm{E}+02$ & 82.69 & $<0.0001$ \\
\hline & oil $\times \Delta T$ & 2 & $7.43 \mathrm{E}+01$ & $3.72 \mathrm{E}+01$ & 13.15 & $<0.0001$ \\
\hline & $o i l \times t$ & 4 & $1.14 \mathrm{E}+03$ & $2.85 \mathrm{E}+02$ & 100.72 & $<0.0001$ \\
\hline & $o i l \times \zeta \times q$ & 2 & $8.15 \mathrm{E}+01$ & $4.08 \mathrm{E}+01$ & 14.42 & $<0.0001$ \\
\hline & oil $\times \zeta \times \Delta T$ & 2 & $2.51 \mathrm{E}+02$ & $1.25 \mathrm{E}+02$ & 44.38 & $<0.0001$ \\
\hline & $o i l \times \zeta \times t$ & 4 & $2.92 \mathrm{E}+01$ & $7.30 \mathrm{E}+00$ & 2.58 & 0.0371 \\
\hline & oil $\times q \times \Delta T$ & 4 & $3.00 \mathrm{E}+02$ & $7.50 \mathrm{E}+01$ & 26.53 & $<0.0001$ \\
\hline & $o i l \times q \times t$ & 8 & $1.67 \mathrm{E}+02$ & $2.08 \mathrm{E}+01$ & 7.36 & $<0.0001$ \\
\hline & $o i l \times \Delta T \times t$ & 8 & $1.56 \mathrm{E}+02$ & $1.96 \mathrm{E}+01$ & 6.92 & $<0.0001$ \\
\hline & $o i l \times \zeta \times q \times \Delta T$ & 4 & $3.25 \mathrm{E}+01$ & $8.12 \mathrm{E}+00$ & 2.87 & 0.0229 \\
\hline & $o i l \times \zeta \times \Delta T \times t$ & 8 & $1.37 \mathrm{E}+02$ & $1.71 \mathrm{E}+01$ & 6.04 & $<0.0001$ \\
\hline & oil $\times q \times \Delta T \times t$ & 16 & $1.00 \mathrm{E}+02$ & $6.26 \mathrm{E}+00$ & 2.22 & 0.0047 \\
\hline & $o i l \times \zeta \times q \times \Delta T \times t$ & 24 & $1.29 \mathrm{E}+02$ & $5.35 \mathrm{E}+00$ & 1.89 & 0.0075 \\
\hline & $\zeta$ & 1 & $3.01 \mathrm{E}+02$ & $3.01 \mathrm{E}+02$ & 106.55 & $<0.0001$ \\
\hline & $\zeta \times q$ & 2 & $3.45 \mathrm{E}+01$ & $1.73 \mathrm{E}+01$ & 6.11 & 0.0025 \\
\hline & $\zeta \times \Delta T$ & 2 & $9.92 \mathrm{E}+02$ & $4.96 \mathrm{E}+02$ & 175.51 & $<0.0001$ \\
\hline & $\zeta \times t$ & 4 & $1.18 \mathrm{E}+02$ & $2.95 \mathrm{E}+01$ & 10.44 & $<0.0001$ \\
\hline & $\zeta \times q \times \Delta T$ & 4 & $1.63 \mathrm{E}+02$ & $4.06 \mathrm{E}+01$ & 14.37 & $<0.0001$ \\
\hline & $\zeta \times q \times t$ & 8 & $8.58 \mathrm{E}+01$ & $1.07 \mathrm{E}+01$ & 3.79 & 0.0003 \\
\hline & $\zeta \times \Delta T \times t$ & 8 & $2.07 \mathrm{E}+02$ & $2.59 \mathrm{E}+01$ & 9.15 & $<0.0001$ \\
\hline & $\zeta \times q \times \Delta T \times t$ & 16 & $1.86 \mathrm{E}+02$ & $1.16 \mathrm{E}+01$ & 4.11 & $<0.0001$ \\
\hline & $q$ & 2 & $5.51 \mathrm{E}+03$ & $2.75 \mathrm{E}+03$ & 973.97 & $<0.0001$ \\
\hline & $q \times \Delta T$ & 4 & $1.18 \mathrm{E}+03$ & $2.96 \mathrm{E}+02$ & 104.64 & $<0.0001$ \\
\hline & $q \times t$ & 8 & $9.68 \mathrm{E}+02$ & $1.21 \mathrm{E}+02$ & 42.81 & $<0.0001$ \\
\hline & $q \times \Delta T \times t$ & 16 & $2.95 \mathrm{E}+02$ & $1.84 \mathrm{E}+01$ & 6.51 & $<0.0001$ \\
\hline & $\Delta T$ & 2 & $6.41 \mathrm{E}+02$ & $3.21 \mathrm{E}+02$ & 113.41 & $<0.0001$ \\
\hline & $\Delta T \times t$ & 8 & $1.12 \mathrm{E}+02$ & $1.40 \mathrm{E}+01$ & 4.96 & $<0.0001$ \\
\hline & $t$ & 4 & $1.77 \mathrm{E}+04$ & $4.41 \mathrm{E}+03$ & 1560.80 & $<0.0001$ \\
\hline Error & & 358 & $1.01 \mathrm{E}+03$ & $2.83 \mathrm{E}+00$ & & \\
\hline Total & & 539 & $3.63 \mathrm{E}+04$ & & & \\
\hline
\end{tabular}

A2.3.1 Degrees of freedom (DF); sum of squares (SS); mean squares (MS). 
Table A2.3.2 ANOVA for $G^{\prime}{ }^{A 2.3 .2}$

\begin{tabular}{|c|c|c|c|c|c|c|}
\hline Source & & DF & SS & MS & $\mathbf{F}$ & $\mathbf{p}$ \\
\hline \multicolumn{7}{|l|}{ Model } \\
\hline & Replicate & 2 & $4.05 \mathrm{E}+01$ & $2.03 \mathrm{E}+01$ & 33.47 & $<0.0001$ \\
\hline & $\omega$ & 20 & $6.45 \mathrm{E}+02$ & $3.23 \mathrm{E}+01$ & 53.33 & $<0.0001$ \\
\hline & oil & 1 & $7.77 \mathrm{E}+03$ & $7.77 \mathrm{E}+03$ & 12840.90 & $<0.0001$ \\
\hline & $o i l \times \zeta$ & 1 & $2.21 \mathrm{E}+02$ & $2.21 \mathrm{E}+02$ & 364.59 & $<0.0001$ \\
\hline & oil $\times q$ & 2 & $6.03 \mathrm{E}+02$ & $3.01 \mathrm{E}+02$ & 498.06 & $<0.0001$ \\
\hline & $o i l \times \Delta T$ & 2 & $4.53 \mathrm{E}+02$ & $2.26 \mathrm{E}+02$ & 374.10 & $<0.0001$ \\
\hline & $o i l \times t$ & 4 & $2.24 \mathrm{E}+03$ & $5.60 \mathrm{E}+02$ & 925.85 & $<0.0001$ \\
\hline & oil $\times \zeta \times q$ & 2 & $2.51 \mathrm{E}+02$ & $1.25 \mathrm{E}+02$ & 207.28 & $<0.0001$ \\
\hline & oil $\times \zeta \times \Delta T$ & 2 & $3.22 \mathrm{E}+02$ & $1.61 \mathrm{E}+02$ & 266.01 & $<0.0001$ \\
\hline & $o i l \times \zeta \times t$ & 4 & $5.81 \mathrm{E}+01$ & $1.45 \mathrm{E}+01$ & 24.00 & $<0.0001$ \\
\hline & oil $\times q \times \Delta T$ & 4 & $4.28 \mathrm{E}+02$ & $1.07 \mathrm{E}+02$ & 176.84 & $<0.0001$ \\
\hline & oil $\times q \times t$ & 8 & $3.61 \mathrm{E}+02$ & $4.52 \mathrm{E}+01$ & 74.62 & $<0.0001$ \\
\hline & $o i l \times \Delta T \times t$ & 8 & $1.79 \mathrm{E}+02$ & $2.23 \mathrm{E}+01$ & 36.87 & $<0.0001$ \\
\hline & $o i l \times \zeta \times q \times \Delta T$ & 4 & $4.10 \mathrm{E}+01$ & $1.03 \mathrm{E}+01$ & 16.94 & $<0.0001$ \\
\hline & $o i l \times \zeta \times q \times t$ & 8 & $1.61 \mathrm{E}+02$ & $2.01 \mathrm{E}+01$ & 33.15 & $<0.0001$ \\
\hline & oil $\times \zeta \times \Delta T \times t$ & 8 & $1.34 \mathrm{E}+02$ & $1.68 \mathrm{E}+01$ & 27.77 & $<0.0001$ \\
\hline & oil $\times q \times \Delta T \times t$ & 16 & $2.82 \mathrm{E}+02$ & $1.76 \mathrm{E}+01$ & 29.13 & $<0.0001$ \\
\hline & $o i l \times \zeta \times q \times \Delta T \times t$ & 16 & $5.34 \mathrm{E}+02$ & $3.34 \mathrm{E}+01$ & 55.17 & $<0.0001$ \\
\hline & $\zeta$ & 1 & $1.11 \mathrm{E}+04$ & $1.11 \mathrm{E}+04$ & 18321.90 & $<0.0001$ \\
\hline & $\zeta \times q$ & 2 & $7.93 \mathrm{E}+02$ & $3.97 \mathrm{E}+02$ & 655.33 & $<0.0001$ \\
\hline & $\zeta \times \Delta T$ & 2 & $2.20 \mathrm{E}+02$ & $1.10 \mathrm{E}+02$ & 181.58 & $<0.0001$ \\
\hline & $\zeta \times t$ & 4 & $3.92 \mathrm{E}+02$ & $9.79 \mathrm{E}+01$ & 161.85 & $<0.0001$ \\
\hline & $\zeta \times q \times \Delta T$ & 4 & $6.34 \mathrm{E}+02$ & $1.59 \mathrm{E}+02$ & 262.05 & $<0.0001$ \\
\hline & $\zeta \times q \times t$ & 8 & $4.34 \mathrm{E}+02$ & $5.42 \mathrm{E}+01$ & 89.55 & $<0.0001$ \\
\hline & $\zeta \times \Delta T \times t$ & 8 & $1.26 \mathrm{E}+03$ & $1.58 \mathrm{E}+02$ & 260.65 & $<0.0001$ \\
\hline & $\zeta \times q \times \Delta T \times t$ & 16 & $7.41 \mathrm{E}+02$ & $4.63 \mathrm{E}+01$ & 76.57 & $<0.0001$ \\
\hline & $q$ & 2 & $4.40 \mathrm{E}+03$ & $2.20 \mathrm{E}+03$ & 3632.40 & $<0.0001$ \\
\hline & $q \times \Delta T$ & 4 & $2.99 \mathrm{E}+02$ & $7.47 \mathrm{E}+01$ & 123.41 & $<0.0001$ \\
\hline & $q \times t$ & 8 & $5.73 \mathrm{E}+02$ & $7.16 \mathrm{E}+01$ & 118.34 & $<0.0001$ \\
\hline & $q \times \Delta T \times t$ & 16 & $9.73 \mathrm{E}+02$ & $6.08 \mathrm{E}+01$ & 100.51 & $<0.0001$ \\
\hline & $\Delta T$ & 2 & $1.08 \mathrm{E}+03$ & $5.42 \mathrm{E}+02$ & 895.01 & $<0.0001$ \\
\hline & $\Delta T \times t$ & 8 & $1.16 \mathrm{E}+03$ & $1.45 \mathrm{E}+02$ & 238.96 & $<0.0001$ \\
\hline & $t$ & 4 & $9.90 \mathrm{E}+03$ & $2.48 \mathrm{E}+03$ & 4091.35 & $<0.0001$ \\
\hline Error & & 11060 & $6.69 \mathrm{E}+03$ & $6.05 \mathrm{E}-01$ & & \\
\hline Total & & 11261 & $5.51 \mathrm{E}+04$ & & & \\
\hline
\end{tabular}

A2.3.2 Degrees of freedom (DF); sum of squares (SS); mean squares (MS). 
Table A2.3.3 ANOVA for $F^{A 2.3 .3}$

\begin{tabular}{|c|c|c|c|c|c|c|}
\hline Source & & DF & SS & MS & $\mathbf{F}$ & p \\
\hline \multicolumn{7}{|l|}{ Model } \\
\hline & Replicate & 2 & $6.71 \mathrm{E}+05$ & $3.35 \mathrm{E}+05$ & 6.30 & 0.0020 \\
\hline & oil & 1 & $5.14 \mathrm{E}+07$ & $5.14 \mathrm{E}+07$ & 965.55 & $<0.0001$ \\
\hline & $o i l \times \zeta$ & 1 & $2.33 \mathrm{E}+06$ & $2.33 \mathrm{E}+06$ & 43.71 & $<0.0001$ \\
\hline & $o i l \times q$ & 2 & $1.11 \mathrm{E}+06$ & $5.55 \mathrm{E}+05$ & 10.42 & $<0.0001$ \\
\hline & $o i l \times t$ & 4 & $1.06 \mathrm{E}+07$ & $2.64 \mathrm{E}+06$ & 49.57 & $<0.0001$ \\
\hline & oil $\times \zeta \times q$ & 2 & $1.55 \mathrm{E}+06$ & $7.73 \mathrm{E}+05$ & 14.51 & $<0.0001$ \\
\hline & $o i l \times \zeta \times t$ & 4 & $1.24 \mathrm{E}+06$ & $3.09 \mathrm{E}+05$ & 5.81 & 0.0001 \\
\hline & $o i l \times \zeta \times q \times \Delta T$ & 12 & $1.35 \mathrm{E}+06$ & $1.12 \mathrm{E}+05$ & 2.11 & 0.0152 \\
\hline & oil $\times \zeta \times q \times t$ & 16 & $2.33 \mathrm{E}+06$ & $1.45 \mathrm{E}+05$ & 2.73 & 0.0004 \\
\hline & $\zeta$ & 1 & $2.76 \mathrm{E}+07$ & $2.76 \mathrm{E}+07$ & 519.14 & $<0.0001$ \\
\hline & $\zeta \times q$ & 2 & $6.59 \mathrm{E}+05$ & $3.30 \mathrm{E}+05$ & 6.19 & 0.0020 \\
\hline & $\zeta \times \Delta T$ & 2 & $3.00 \mathrm{E}+06$ & $1.50 \mathrm{E}+06$ & 28.21 & $<0.0001$ \\
\hline & $\zeta \times t$ & 4 & $6.12 \mathrm{E}+06$ & $1.53 \mathrm{E}+06$ & 28.73 & $<0.0001$ \\
\hline & $\zeta \times q \times \Delta T$ & 4 & $1.30 \mathrm{E}+06$ & $3.25 \mathrm{E}+05$ & 6.11 & $<0.0001$ \\
\hline & $\zeta \times q \times t$ & 8 & $1.91 \mathrm{E}+06$ & $2.39 \mathrm{E}+05$ & 4.48 & $<0.0001$ \\
\hline & $q$ & 2 & $1.48 \mathrm{E}+07$ & $7.40 \mathrm{E}+06$ & 138.98 & $<0.0001$ \\
\hline & $q \times \Delta T$ & 4 & $1.41 \mathrm{E}+06$ & $3.52 \mathrm{E}+05$ & 6.61 & $<0.0001$ \\
\hline & $q \times t$ & 8 & $1.71 \mathrm{E}+06$ & $2.14 \mathrm{E}+05$ & 4.02 & 0.0001 \\
\hline & $q \times \Delta T \times t$ & 16 & $1.86 \mathrm{E}+06$ & $1.16 \mathrm{E}+05$ & 2.18 & 0.0052 \\
\hline & $\Delta T$ & 2 & $3.12 \mathrm{E}+06$ & $1.56 \mathrm{E}+06$ & 29.35 & $<0.0001$ \\
\hline & $\Delta T \times t$ & 8 & $8.46 \mathrm{E}+05$ & $1.06 \mathrm{E}+05$ & 1.99 & 0.0467 \\
\hline & $t$ & 4 & $1.14 \mathrm{E}+08$ & $2.85 \mathrm{E}+07$ & 534.93 & $<0.0001$ \\
\hline Error & & 430 & $2.29 \mathrm{E}+07$ & $5.32 \mathrm{E}+04$ & & \\
\hline Total & & 539 & $2.74 \mathrm{E}+08$ & & & \\
\hline
\end{tabular}

A2.3.3 Degrees of freedom (DF); sum of squares (SS); mean squares (MS). 


\section{A3 Multiple Regression Analysis}

\section{A3.1 Sample Calculation}

The models generated within this thesis were third-order as it was determined that the unexplained variance associated with second-order models was too large. Additionally, little gain in this variance was to be had as the models increased from third- to fourth-order.

The process of reducing a full model into a subset model using Mallows' $C_{P}$ statistic as a guide is purely computational as thousands of subset permutations may be compared, depending on the total number of parameters in the original full model (Mallows, 1973). Therefore, this sample calculation begins with the subset model and follows the formulas provided in Snedecor and Cochran (1989). A basic knowledge of matrix notation and algebra is also required to understand how partial regression coefficients of these subset models are derived.

Subset model 3.9b from Chapter 3 which predicted firmness in PMF was used for this sample calculation simply for its brevity compared to the other models. The associated temporary model of this subset, before coefficient derivation, is:

$$
\hat{\mathrm{y}}=\beta_{0}+\beta_{1} t+\beta_{2} t^{2}+\beta_{3} t^{3}+\beta_{4} \Delta T t+\beta_{5} \Delta T t^{2}+\beta_{6} \zeta t
$$

where $\beta$ values are partial regression coefficients. This model was composed from the dataset provided in Table A3.1.1 with a total $(n)$ of 90 observations.

To estimate the $\beta$ values, the principle of least squares was employed. The least squares equation was written as the matrix notation: 
Table A3.1.1 Firmness dataset for PMF (Chapter 3). ${ }^{A 3.1 .1}$

\begin{tabular}{|c|c|c|c|c|c|}
\hline$\zeta$ & $\Delta T\left({ }^{\circ} \mathrm{C}\right)$ & $t$ (week) & & F (kg) & \\
\hline \multirow{15}{*}{0} & \multirow{5}{*}{-5} & 0 & 0.002 & 0.002 & 0.002 \\
\hline & & 1 & 0.194 & 0.177 & 0.253 \\
\hline & & 2 & 0.365 & 0.328 & 0.319 \\
\hline & & 3 & 0.474 & 0.417 & 0.406 \\
\hline & & 4 & 0.592 & 0.483 & 0.494 \\
\hline & \multirow{5}{*}{0} & 0 & 0.015 & 0.006 & 0.009 \\
\hline & & 1 & 0.513 & 0.427 & 0.437 \\
\hline & & 2 & 0.666 & 0.457 & 0.597 \\
\hline & & 3 & 0.624 & 0.562 & 0.673 \\
\hline & & 4 & 0.506 & 0.532 & 0.585 \\
\hline & \multirow{5}{*}{8} & 0 & 0.034 & 0.010 & 0.030 \\
\hline & & 1 & 0.562 & 0.552 & 0.642 \\
\hline & & 2 & 0.558 & 0.635 & 0.704 \\
\hline & & 3 & 0.863 & 0.729 & 0.729 \\
\hline & & 4 & 0.748 & 0.838 & 0.812 \\
\hline \multirow{15}{*}{1} & \multirow{5}{*}{-5} & 0 & 0.007 & 0.005 & 0.008 \\
\hline & & 1 & 0.170 & 0.500 & 0.269 \\
\hline & & 2 & 0.396 & 0.637 & 0.390 \\
\hline & & 3 & 0.340 & 0.618 & 0.535 \\
\hline & & 4 & 0.611 & 0.750 & 0.645 \\
\hline & \multirow{5}{*}{0} & 0 & 0.004 & 0.004 & 0.005 \\
\hline & & 1 & 0.661 & 0.258 & 0.392 \\
\hline & & 2 & 0.936 & 0.449 & 0.554 \\
\hline & & 3 & 0.959 & 0.552 & 0.788 \\
\hline & & 4 & 1.126 & 0.654 & 0.878 \\
\hline & \multirow{5}{*}{8} & 0 & 0.032 & 0.055 & 0.075 \\
\hline & & 1 & 0.470 & 0.880 & 0.373 \\
\hline & & 2 & 0.764 & 0.968 & 0.530 \\
\hline & & 3 & 0.996 & 1.324 & 0.603 \\
\hline & & 4 & 0.895 & 1.310 & 0.592 \\
\hline
\end{tabular}

A3.1.1 Confectioner's sugar ( $\zeta)$; cooling endpoint deviation $(\Delta T)$; storage time $(t)$, firmness (F). 


$$
\left(X^{T} \cdot X\right) b=\left(X^{T} \cdot Y\right)
$$

where $\left(X^{T} \cdot X\right)$ is the symmetric matrix further defined as:

$$
\begin{aligned}
& \left(\mathrm{X}^{\mathrm{T}} \cdot \mathrm{X}\right)=\left|\begin{array}{ccccccc}
\mathrm{n} & \Sigma \mathrm{t} & \Sigma \mathrm{t}^{2} & \Sigma \mathrm{t}^{3} & \Sigma \Delta \mathrm{Tt} & \Sigma \Delta \mathrm{Tt}^{2} & \Sigma \zeta \mathrm{t} \\
\Sigma \mathrm{t} & \Sigma \mathrm{t}^{2} & \Sigma \mathrm{t}^{3} & \Sigma \mathrm{t}^{4} & \Sigma \Delta \mathrm{Tt}^{2} & \Sigma \Delta \mathrm{Tt}^{3} & \Sigma \zeta \mathrm{t}^{2} \\
\Sigma \mathrm{t}^{2} & \Sigma \mathrm{t}^{3} & \Sigma \mathrm{t}^{4} & \Sigma \mathrm{t}^{5} & \Sigma \Delta \mathrm{Tt}^{3} & \Sigma \Delta \mathrm{Tt}^{4} & \Sigma \zeta \mathrm{t}^{3} \\
\Sigma \mathrm{t}^{3} & \Sigma \mathrm{t}^{4} & \Sigma \mathrm{t}^{5} & \Sigma \mathrm{t}^{6} & \Sigma \Delta \mathrm{Tt}^{4} & \Sigma \Delta \mathrm{Tt}^{5} & \Sigma \zeta \mathrm{t}^{4} \\
\Sigma \Delta \mathrm{Tt} & \Sigma \Delta \mathrm{Tt}^{2} & \Sigma \Delta \mathrm{Tt}^{3} & \Sigma \Delta \mathrm{Tt}^{4} & \Sigma \Delta \mathrm{T}^{2} \mathrm{t}^{2} & \Sigma \Delta \mathrm{T}^{2} \mathrm{t}^{3} & \Sigma \zeta \Delta \mathrm{Tt}^{2} \\
\Sigma \Delta \mathrm{Tt}^{2} & \Sigma \Delta \mathrm{Tt}^{3} & \Sigma \Delta \mathrm{Tt}^{4} & \Sigma \Delta \mathrm{Tt}^{5} & \Sigma \Delta \mathrm{T}^{2} \mathrm{t}^{3} & \Sigma \Delta \mathrm{T}^{2} \mathrm{t}^{4} & \Sigma \zeta \Delta \mathrm{Tt}^{3} \\
\Sigma \zeta \mathrm{t} & \Sigma \zeta \mathrm{t}^{2} & \Sigma \zeta \mathrm{t}^{3} & \Sigma \zeta \mathrm{t}^{4} & \Sigma \zeta \Delta \mathrm{Tt}^{2} & \Sigma \zeta \Delta \mathrm{Tt}^{3} & \Sigma \zeta^{2} \mathrm{t}^{2}
\end{array}\right| \\
& \left(\mathrm{X}^{\mathrm{T}} \cdot \mathrm{X}\right)=\left|\begin{array}{ccccccc}
90 & 180 & 540 & 1800 & 180 & 540 & 90 \\
180 & 540 & 1800 & 6372 & 540 & 1800 & 270 \\
540 & 1800 & 6372 & 23400 & 1800 & 6372 & 900 \\
1800 & 6372 & 23400 & 88020 & 6372 & 23400 & 3186 \\
180 & 540 & 1800 & 6372 & 16020 & 53400 & 270 \\
540 & 1800 & 6372 & 23400 & 53400 & 189036 & 900 \\
90 & 270 & 900 & 3186 & 270 & 900 & 270
\end{array}\right|
\end{aligned}
$$

and $\left(X^{T} \cdot Y\right)$ is further defined as:

$$
\begin{gathered}
\left(\mathrm{X}^{\mathrm{T}} \cdot \mathrm{Y}\right)=\left|\begin{array}{c}
\Sigma \mathrm{y} \\
\Sigma \mathrm{ty} \\
\Sigma \mathrm{t}^{2} \mathrm{y} \\
\Sigma \mathrm{t}^{3} \mathrm{y} \\
\Sigma \Delta \mathrm{Tty} \\
\Sigma \Delta \mathrm{Tt}^{2} \mathrm{y} \\
\Sigma \zeta \mathrm{ty}
\end{array}\right| \\
\left(\mathrm{X}^{\mathrm{T}} \cdot \mathrm{Y}\right)=\left|\begin{array}{c}
43.526 \\
117.012 \\
367.280 \\
1254.191 \\
240.981 \\
735.498 \\
65.213
\end{array}\right|
\end{gathered}
$$


To estimate the partial regression coefficients for model $3.9 \mathrm{~b}$, the least squares equation was solved for its corresponding matrix $(b)$ :

$$
\mathrm{b}=\left(\mathrm{X}^{\mathrm{T}} \cdot \mathrm{X}\right)^{-1}\left(\mathrm{X}^{\mathrm{T}} \cdot \mathrm{Y}\right)=\left|\begin{array}{c}
\mathrm{b}_{0} \\
\mathrm{~b}_{1} \\
\mathrm{~b}_{2} \\
\mathrm{~b}_{3} \\
\mathrm{~b}_{4} \\
\mathrm{~b}_{5} \\
\mathrm{~b}_{6}
\end{array}\right|
$$

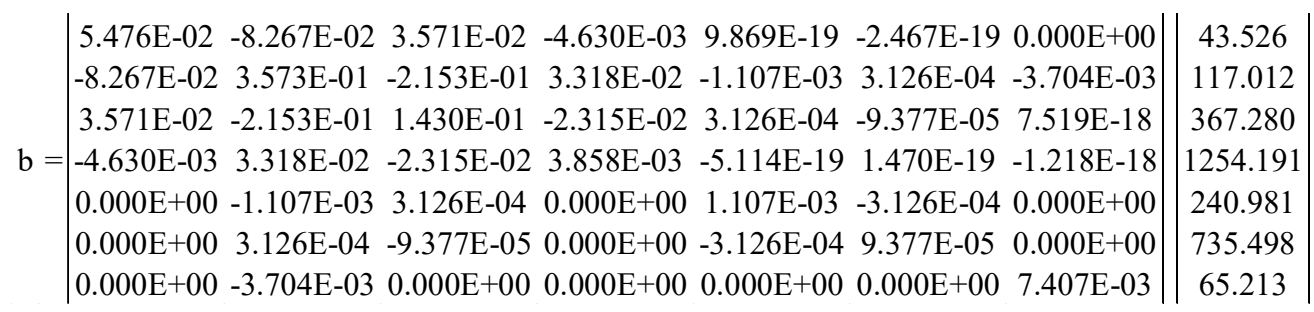

$\mathrm{b}=\left|\begin{array}{c}0.0206 \\ 0.4894 \\ -0.1563 \\ 0.0177 \\ 0.0221 \\ -0.0042 \\ 0.0497\end{array}\right|$




\section{A3.2 Confidence Limits}

Continuing with model $3.9 \mathrm{~b}$ as an example, its variance of error $\left(\sigma^{2}\right)$ was first determined to later predict the confidence limits. This began with the determination of its total sum of squares as shown in Table A3.2.1 where y and ŷ represent the observed and predicted (based on model 3.9b) firmness for PMF.

Table A3.2.1 Sum of squares for PMF (Chapter 3).

\begin{tabular}{|c|c|c|c|}
\hline Observation & y (kg) & $\hat{\mathbf{y}} \mathbf{( k g )}$ & $(y-\hat{y})^{2}\left(k^{2}\right)$ \\
\hline 1 & 0.0020 & 0.0206 & $3.470 \mathrm{E}-04$ \\
\hline 2 & 0.0017 & 0.0206 & $3.583 \mathrm{E}-04$ \\
\hline 3 & 0.0017 & 0.0206 & $3.583 \mathrm{E}-04$ \\
\hline 4 & 0.1940 & 0.2821 & $7.758 \mathrm{E}-03$ \\
\hline 5 & 0.1773 & 0.2821 & $1.098 \mathrm{E}-02$ \\
\hline 6 & 0.2530 & 0.2821 & $8.456 \mathrm{E}-04$ \\
\hline 7 & 0.3646 & 0.3792 & $2.125 \mathrm{E}-04$ \\
\hline 8 & 0.3276 & 0.3792 & $2.660 \mathrm{E}-03$ \\
\hline 9 & 0.3187 & 0.3792 & $3.658 \mathrm{E}-03$ \\
\hline 10 & 0.4741 & 0.4181 & $3.141 \mathrm{E}-03$ \\
\hline$\vdots$ & $\vdots$ & $\vdots$ & $\vdots$ \\
\hline 88 & 0.8949 & 0.9737 & $6.206 \mathrm{E}-03$ \\
\hline 89 & 1.3097 & 0.9737 & $1.129 \mathrm{E}-01$ \\
\hline 90 & 0.5920 & 0.9737 & $1.457 \mathrm{E}-01$ \\
\hline Total & & & $1.620 \mathrm{E}+00$ \\
\hline
\end{tabular}

The total degrees of freedom for the dataset was 89 (e.g., $n-1)$ whereas the degrees of freedom for the model was 6 (e.g., total parameters minus one). Consequently, the degrees of freedom that was associated with error was 83 . With this information, $\sigma^{2}$ was determined to be:

$$
\sigma^{2}=\frac{\sum(y-\hat{y})^{2}}{d f}=\frac{1.620}{83}=0.01952
$$


To estimate the confidence limits around an arbitrary point (e.g., $\zeta=0, q=10^{\circ} \mathrm{C} \cdot \mathrm{min}^{-1}$, $\Delta T=0{ }^{\circ} \mathrm{C}$, and $t=4$ weeks), $\hat{y}$ was first determined from the subset model:

$$
\begin{gathered}
\hat{y}=0.0206+0.4894(4)-0.1563(4)^{2}+0.0177(4)^{3}+0.0221(0)(4) \\
-0.0042(0)(4)^{2}+(0)[0.0497(0)] \\
\hat{y}=0.610
\end{gathered}
$$

Next, the variance associated with this arbitrary point was determined:

$$
\operatorname{Var}(\hat{y})=c^{T}\left(X^{T} \cdot X\right)^{-1} c \sigma^{2}
$$

where $c^{T}$ was further defined as:

$$
\mathrm{c}^{\mathrm{T}}=\left|1 \mathrm{t} \mathrm{t}^{2} \mathrm{t}^{3} \Delta \mathrm{Tt} \Delta \mathrm{Tt}^{2} \zeta \mathrm{t}\right|
$$

Therefore:

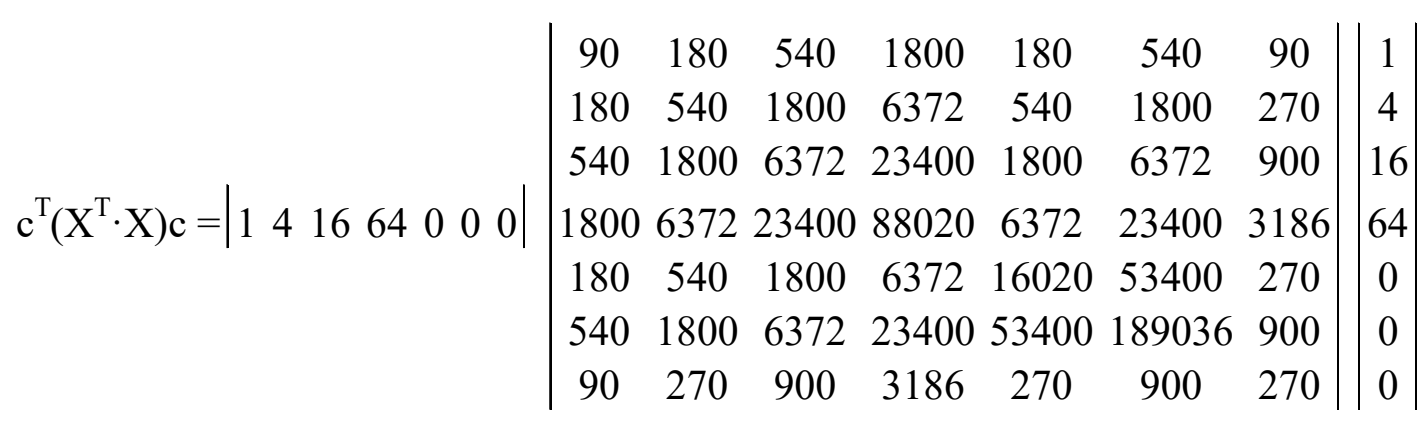

$$
\begin{aligned}
& c^{T}\left(X^{T} \cdot X\right)^{-1} c=0.08609 \\
& \operatorname{Var}(\hat{y})=c^{T}\left(X^{T} \cdot X\right)^{-1} c \sigma^{2}=(0.08609)(0.01952)=0.00168
\end{aligned}
$$

With $\hat{y}$ and $\operatorname{Var}(\hat{y})$ solved, the confidence limits were determined for a two-tailed test $(\alpha=$ $0.05)$ according to: 


$$
\begin{gathered}
(L C L, U C L)=\hat{\mathrm{y}} \pm t_{d f, \frac{\alpha}{2}} \times \sqrt{\operatorname{Var}(\hat{\mathrm{y}})} \\
(L C L, U C L)=0.610 \pm(1.989)(\sqrt{0.00168})=(0.607,0.614)
\end{gathered}
$$




\section{A4 Crystal Morphology of Palm Mid-Fraction}
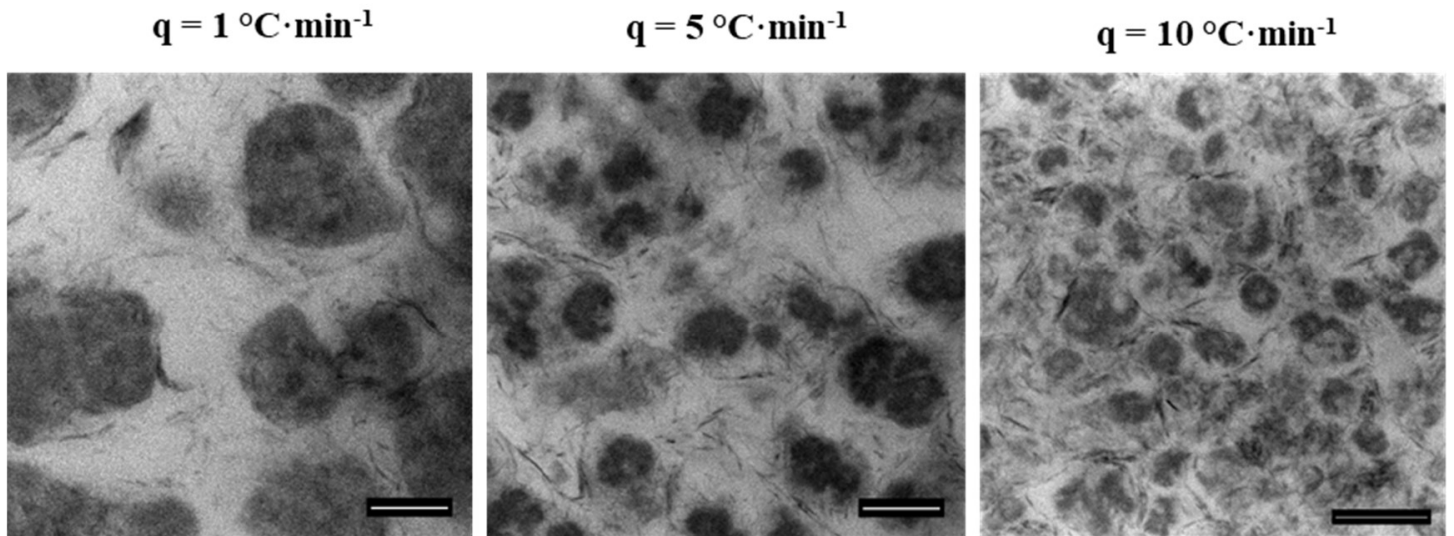

II
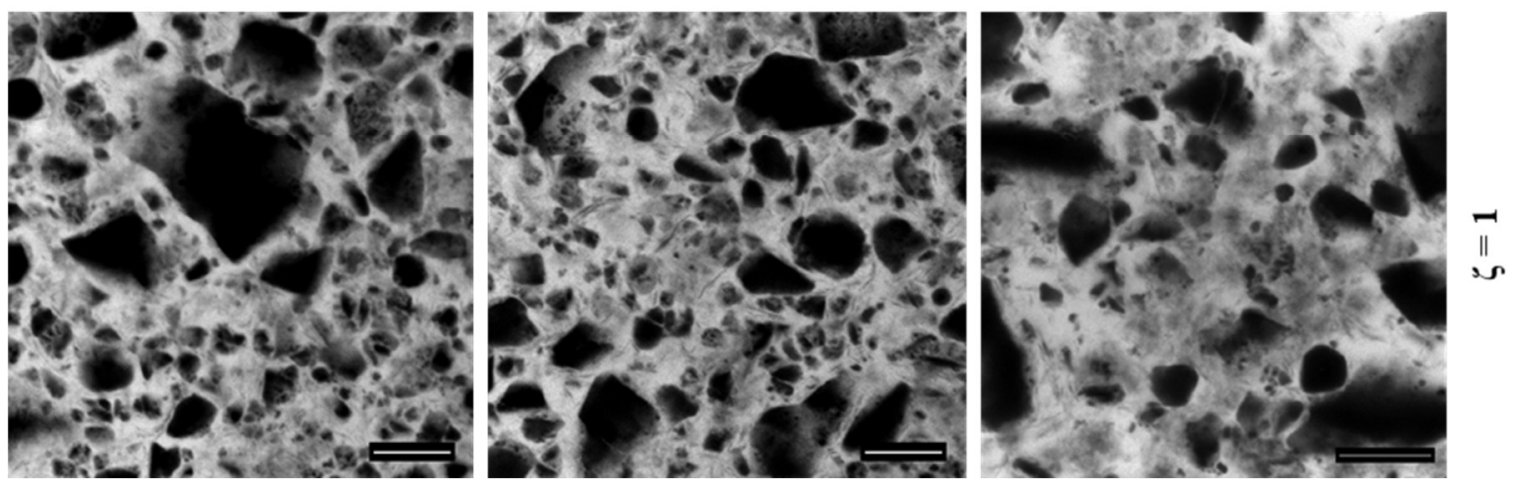

Figure A4.1 Effects of sugar and cooling rate on final PMF crystal morphology (e.g., week 4) cooled to $20^{\circ} \mathrm{C}(\mathrm{bar}=20 \mu \mathrm{m})$. 


\section{References}

Ab Latip, R., Lee, Y.-Y., Tang, T.-K., Phuah, E.-T., Lee, C.-M., Tan, C.-P., and Lai, O.-M. (2013). Palm-based diacylglycerol fat dry fractionation: Effect of crystallisation temperature, cooling rate, and agitation speed on physical and chemical properties of fractions. PeerJ. 1:e72.

Acevedo, N.C. and Marangoni, A.G. (2010). Characterization of the nanoscale in triacylglycerol crystal networks. Cryst. Growth Des. 10(8):3327-3333.

Acevedo, N.C., Peyronel, F., and Marangoni, A.G. (2011). Nanoscale structure intercrystalline interactions in fat crystal networks. Curr. Opin. Colloid Interface Sci. 16:374-383.

Afoakwa, E.O., Paterson, A., and Fowler, M. (2007). Factors influencing rheological and textural qualities in chocolate: A review. Trends Food Sci. Technol. 18(6):290-298.

Afoakwa, E.O., Paterson, A., Fowler, M., and Veira, J. (2008a). Characterization of melting properties in dark chocolate from varying particle size distribution and composition using differential scanning calorimetry. Food Res. Int. 41(7):751-757.

Afoakwa, E.O., Paterson, A., and Fowler, M. (2008b). Effects of particle size distribution and composition on rheological properties of dark chocolate. Eur. Food Res. Technol. 226(2):1259-1268.

Afoakwa, E.O., Paterson, A., Fowler, M., and Ryan, A. (2008c). Flavor formation and character in cocoa and chocolate: A critical review. Crit. Rev. Food Sci. Nutr. 48(9):1-18.

Afoakwa, E.O., Paterson, A., Fowler, M., and Vieira, J. (2008d). Effects of tempering and fat crystallization behaviours on microstructure, mechanical properties, and appearance in dark chocolate systems. J. Food Eng. 89(2):128-136.

Afoakwa, E.O., Paterson, A., Fowler, M., and Vieira, J. (2009). Influence of tempering and fat crystallization behaviours on microstructural and melting properties in dark chocolate systems. Food Res. Int. 42(1):200-209. 
Aftab, A.K., Sherazi, S.T.H., Rubina, S., Razia, S., Ambrat, and Arfa, Y. (2013). Consequence of fatty acids profile including trans fat in chocolate and pastry samples. Int. Food Res. J. 20(2):601-605.

Aguilera, J.M., Michel, M., and Mayor, G. (2004). Fat migration in chocolate: Diffusion or capillary flow in a particulate solid? - A hypothesis paper. J. Food Sci. 69(7):169-174.

Aguilera, J.M. and Stanley, D.W. (1999). Microstructural Principles of Food Processing and Engineering. Aspen Publishers, Gaithersburg, Maryland, U.S.A.

Ahmed, J. and Ramaswamy, H.S. (2006). Viscoelastic properties of sweet potato puree infant food. J. Food Eng. 74(3):376-382.

Aidoo, H., Sakyi-Dawson, E., Abbey, L., Tano-Debrah, K., and Saalia, F.K. (2012). Optimisation of chocolate formulation using dehydrated peanut-cowpea milk to replace dairy milk. J. Sci. Food Agric. 92(2):224-231.

Aidoo, R.P., Depypere, F., Afoakwa, E.O., and Dewettinck, K. (2013). Industrial manufacture of sugar-free chocolates: Applicability of alternative sweeteners and carbohydrate polymers as raw materials in product development. Trends Food Sci. Technol. 32(2):84-96.

Ali, A., Selamat, J., Che Man, Y.B., and Suria, A.M. (2001). Effect of storage temperature on texture, polymorphic structure, bloom formation and sensory attributes of filled dark chocolate. Food Chem. 72(4):491-497.

Allen, S.L. (2002). In the Devil's Garden: A Sinful History of Forbidden Food. Random House, New York City, New York, U.S.A.

Altimiras, P., Pyle, L., and Bouchon, P. (2007). Structure-fat migration relationships during storage of cocoa butter model bars: Bloom development and possible mechanisms. J. Food Eng. 80(2):600-610.

Ascherio, A., Katan, M.B., Zock, P.L., Stampfer, M.J., and Willett, W.C. (1999). Trans fatty acids and coronary heart disease. N. Eng. J. Med. 340(25):1994-1998. 
Awua, P.K. (2002). Cocoa Processing and Chocolate Manufacture in Ghana. David Jamieson and Associates Press, Essex, U.K.

Baixauli, R., Sanz, T., Salvador, A., and Fiszman, S.M. (2007). Influence of the dosing process on the rheological and microstructural properties of a bakery product. Food Hydrocolloid. 21(2):230-236.

Baldino, N., Gabriele, D., and Migliori, M. (2010). The influence of formulation and cooling rate on the rheological properties of chocolate. Eur. Food Res. Technol. 231(6):821-828.

Barron, I. F. (1977). The expansion of wafer and its relation to the cracking of chocolate and 'bakers' chocolate coatings. Int. J. Food Sci. Technol. 12(1):73-84.

Basiron, Y. (2001). New palm-based products. J. Oleo Sci. 50(5):295-303.

Basso, R., Ribeiro, A., Masuchi, M., Gioielli, L., Gonçalves, L., Cardoso, L., and Grimaldi, R. (2010). Tripalmitin and monoacylglycerols as modifiers in the crystallisation of palm oil. Food Chem. 122(4):1185-1192.

Beckett, S.T. (1994). Control of particle size reduction during chocolate grinding. In: Proceedings of the Pennsylvania Manufacturing Confectioners' Association Production Conference, pp. 136-143. PMCA, Center Valley, Pennsylvania, U.S.A.

Beckett, S.T. (1999). Preparation of Chocolate with Limonene to Reduce Fat Content. United States Patent 6,200,625.

Beckett, S.T. (2000). The Science of Chocolate. Royal Society of Chemistry, Cambridge, U.K.

Beckett, S.T. (2008). Industrial Chocolate Manufacture and Use. Blackwell Science, Oxford, U.K.

Bennett, A., Hamilton, D., Maradudin, A., Miller, R., and Murphy, J. (1965). Crystals: Perfect and Imperfect. Walker and Co., New York City, New York, U.S.A.

Bigalli, G.L. (1988). Practical aspects of the eutectic effect on confectionery fats and their mixtures. Manuf. Confect. 68:65-80. 
Binnig, G., Quate, C.F., and Gerber, C. (1986). Atomic force microscope. Phys. Rev. Lett. 56(9):930-933.

Blanchette, C.D., Loui, A., and Ratto, T.V. (2008). Tip functionalization: Applications to chemical force spectroscopy. In: Handbook of Molecular Force Spectroscopy, pp. 185-203. Noy, A., Ed., Springer Science+Business Media, New York City, New York, U.S.A.

Bomba, P.C. (1993). Shelf life of chocolate confectionery products. In: Shelf Life Studies of Foods and Beverages, pp. 341-351. Charalambous, G., Ed., Elsevier, London, U.K.

Bourne, M. (2002). Food Texture and Viscosity: Concepts and Measurements. Academic Press, San Diego, California, U.S.A.

Bouzas, J. and Brown, B.D. (1995). Interacting affecting microstructure, texture, and rheology of chocolate confectionery products. In: Ingredient Interactions: Effects on Food Quality, pp. 451-528. Gaonkar, A. G., Ed., Marcel and Dekker, New York City, New York, U.S.A.

Bowser, A. (2006). Crystallization of cocoa butter. Manuf. Confect. 86:115-118.

Bresson, S., El Marssi, M., and Khelifa, B. (2005). Raman spectroscopy investigation of various saturated monoacid triglycerides. Chem. Phys. Lipids 134(2):119-129.

Bresson, S., El Marssi, M., and Khelifa, B. (2006). Conformational influences of the polymorphic forms on the $\mathrm{CO}$ and $\mathrm{C}-\mathrm{H}$ stretching modes of five saturated monoacid triglycerides studied by Raman spectroscopy at various temperatures. Vib. Spectrosc. 40(2):263-269.

Bresson, S., Rousseau, D., Ghosh, S., El Marssi, M., and Faivre, V. (2011). Raman spectroscopy of the polymorphic forms and liquid state of cocoa butter. Eur. J. Lipid Sci. Technol. 113(8):992-1004.

Bricknell, J. and Hartel, R.W. (1998). Relation of fat bloom in chocolate to polymorphic transition of cocoa butter. J. Am. Oil Chem. Soc. 75(11):1609-1615. 
Briones, V. and Aguilera, J.M. (2005). Image analysis of changes in surface colour of chocolate. Food Res. Int. 38(1):87-94.

Brown, C.A., Charles, P.D., Johnsen, W.A., and Chesters, S. (1993). Fractal analysis of topographic data by the patchwork method. Wear 161(1-2):61-67.

Bustamante, C., Smith, S.B., Liphardt, J., and Smith, D. (2000). Single-molecule studies of DNA mechanics. Curr. Opin. Struct. Biol. 10(3):279-285.

Cacciuto, A., Auer, S., and Frenkel, D. (2004). Onset of heterogeneous crystal nucleation in colloidal suspensions. Nature 428(6981):404-406.

Campbell, L. (1967). Fat bloom on Chocolate and Its Control by Tempering. British Food Manufacturing Industries Research Association, Leatherhead, U.K.

Campos, R., Narine, S.S., and Marangoni, A.G. (2002). Effect of cooling rate on the structure and mechanical properties of milk fat and lard. Food Res. Int. 35(10):971-981.

Cappella, B. and Dietler, G. (1999). Force-distance curves by atomic force microscopy. Surf. Sci. Rep. 34(1-2):1-104.

Carrion-Vazquez, M., Li, H., Lu, H., Marszalek, P.E., Oberhauser, A.F., and Fernandez, J.M. (2003). The mechanical stability of ubiquitin is linkage dependent. Nat. Struct. Biol. 10(9):738-743.

Carrion-Vazquez, M., Marszalek, P.E., Oberhauser, A.F., and Fernandez, J.M. (1999). Atomic force microscopy captures length phenotypes in single proteins. Proc. Natl. Acad. Sci. U.S.A. 96(20):11288-11292.

Carvalho-da-Silva, A.M., Van Damme, I., Taylor, W., Hort, J., and Wolf, B. (2013). Oral processing of two milk chocolate samples. Food Funct. 4(3):461-469.

Carvalho-da-Silva, A.M., Van Damme, I., Wolf, B., and Hort, J. (2011). Characterisation of chocolate eating behaviour. Physiol. Behav. 104(5):929-933.

Cebula, D.J. and Ziegleder, G. (1993). Studies of bloom formation using x-ray diffraction from chocolates after long-term storage. Eur. J. Lipid Sci. Technol. 95(9):340-343. 
Che Man, Y.B., Haryati, T., Ghazali, H.M., and Asbi, B.A. (1999). Composition and thermal profile of crude palm oil and its products. J. Am. Oil Chem. Soc. 76(2):237-242.

Chen, C.E., Lai, O.M., Ghazali, H.M., and Chong, C.L. (2002). Isothermal crystallization kinetics of refined palm oil. J. Am. Oil Chem. Soc. 79(4):403-410.

Chevalley, J. (1999). Chocolate flow properties. In: Industrial Chocolate Manufacture and Use, pp. 182-200. Beckett, S.T., Ed., Blackwell Science, Oxford, U.K.

Chhabra, R.P. (2006). Bubbles, Drops, and Particles in Non Newtonian Fluids. CRC Press, Boca Raton, Louisiana, U.S.A.

Chong, C.L. and Sambanthamurthi, R. (1993). Effects of mesocarp bruising on the rate of free fatty acid release in oil palm fruits. Int. Biodeterior. Biodegradation 31(1):65-70.

Clayton, P. and Conn, H. (2005). Carbohydrate Substitutes. International Patent Cooperation Treaty, Number WO 2005/006891 A1.

Cook, L.R. (1964). Chocolate Production and Use. Harcourt Brace, New York City, New York, U.S.A.

Costales-Rodríguez, R., Gibon, V., Verhé, R., and De Greyt, W. (2009). Chemical and enzymatic interesterification of a blend of palm stearin:soybean oil for low trans-margarine formulation. J. Am. Oil Chem. Soc. 86(7):681-697.

Cottrell, R.C. (1991). Nutritional aspects of palm oil. Am. J. Clin. Nutr. 53(4 Suppl):989S1009S.

Coutinho, J.A.P., Lopes da Silva, J.A., Ferreira, A., Soares, M.R., and Daridon, J.-L. (2003). Evidence for the aging of wax deposits in crude oils by Ostwald ripening. Pet. Sci. Technol. 21(3-4):381-391.

Couzens, P.J. and Wille, H.J. (1997). Fat migration in composite confectionery products. Manuf. Confect. 77:45-47.

Cox, W.P. and Merz, E.H. (1958). Correlation of dynamic and steady flow viscosities. $J$. Polym. Sci. 28(118):619-622. 
D’Souza, V., DeMan, J.M., and DeMan, L. (1990). Short spacings and polymorphic forms of natural and commercial solid fats: A review. J. Am. Oil Chem. Soc. 67(11):835-843.

Da Silva, E., Bresson, S., and Rousseau, D. (2009). Characterization of the three major polymorphic forms and liquid state of tristearin by Raman spectroscopy. Chem. Phys. Lipids 157(2):113-119.

Dahlenborg, H. (2014). Fat Bloom on Chocolate Confectionery Systems. Lund University, Lund, Sweden.

Dahlenborg, H., Millqvist-Fureby, A., and Bergenståhl, B. (2015). Effect of particle size in chocolate shell on oil migration and fat bloom development. J. Food Eng. 146:172-181.

Dahlenborg, H., Millqvist-Fureby, A., Bergenståhl, B., and Kalnin, D.J.E. (2011). Investigation of chocolate surfaces using profilometry and low vacuum scanning electron microscopy. J. Am. Oil Chem. Soc. 88(6):773-783.

Dahlenborg, H., Millqvist-Fureby, A., Brandner, B.D., and Bergenståhl, B. (2012). Study of the porous structure of white chocolate by confocal Raman microscopy. Eur. J. Lipid Sci. Technol. 114(8):919-926.

De Cock, N. (2011). Structure Development in Confectionery Products: Importance of Triacylglycerol Composition. Ghent University, Ghent, Belgium.

De Graef, V., Depypere, F., Minnaert, M., and Dewettinck, K. (2011). Chocolate yield stress as measured by oscillatory rheology. Food Res. Int. 44(9):2660-2665.

De Graef, V., Dewettinck, K., Verbeken, D., and Foubert, I. (2006). Rheological behaviour of crystallizing palm oil. Eur. J. Lipid Sci. Technol. 108(10):864-870.

Debaste, F., Kegelaers, Y., Liégeois, S., Ben Amor, H., and Halloin, V. (2008). Contribution to the modelling of chocolate tempering process. J. Food Eng. 88(4):568-575.

DeMan, J. M. (1992). X-ray diffraction spectroscopy in the study of fat polymorphism. Food Res. Int. 25(6):471-476. 
Denker, M., Parat-Wilhelms, M., Drichelt, G., Paucke, J., Luger, A., Borcherding, K., Hottman, W., and Steinhart, H. (2006). Investigation of the retronasal flavour release during the consumption of coffee with additions of milk constituents by 'Oral Breath Sampling.' Food Chem. 98(2):201-208.

Dhonsi, D. and Stapley, A.G.F. (2006). The effect of shear rate, temperature, sugar, and emulsifier on the tempering of cocoa butter. J. Food Eng. 77(4):936-942.

Dian, N.L., Sundram, K., and Idris, N.A. (2006). DSC study on the melting properties of palm oil, sunflower oil, and palm kernel olein blends before and after chemical interesterification. J. Am. Oil Chem. Soc. 83(8):739-745.

Dibildox-Alvarado, E., Rodrigues, J.N., Gioielli, L.A., Toro-Vazquez, J.F., and Marangoni, A.G. (2004). Effects of crystalline microstructure on oil migration in a semisolid fat matrix. Cryst. Growth Des. 4(4):731-736.

Dibildox-Alvarado, E., Laredo, T., Toro-Vazquez, J.F., and Marangoni, A. G. (2010). Prenucleation structuring of TAG melts revealed by fluorescence polarization spectroscopy and molecular mechanics simulations. J. Am. Oil Chem. Soc. 87(10):1115-1125.

Dietz, H., Berkemeier, F., Bertz, M., and Rief, M. (2006). Anisotropic deformation response of single protein molecules. Proc. Natl. Acad. Sci. U.S.A. 103(34):12724-12728.

Do, T.-A.L., Hargreaves, J.M., Wolf, B., Hort, J., and Mitchell, J.R. (2007). Impact of particle size distribution on rheological and textural properties of chocolate models with reduced fat content. J. Food Sci. 72(9):E541-E552.

Do, T.-A. L., Vieira, J., Hargreaves, J.M., Wolf, B., and Mitchell, J.R. (2008). Impact of limonene on the physical properties of reduced fat chocolate. J. Am. Oil Chem. Soc. 85(10):911-920.

Dóka, O., Prágai, E., Bicanic, D., Kylcsár, R., and Ajtony, Z. (2013). Colorimetry and photoacoustic spectroscopy as a suitable tool for determination of fat-free cocoa solids in dark chocolates. Eur. Food Res. Technol. 236(6):963-968. 
Du, H., Kim, C., Corradini, M.G., Ludescher, R.D., and Rogers, M.A. (2014). Microviscosity of liquid oil confined in colloidal fat crystal networks. Soft Matter 10(43):86528658.

Ducker, W.A., Senden, T.J., and Pashley, R.M. (1991). Direct measurement of colloidal forces using an atomic force microscope. Nature 353(6341):239-241.

Ebner, A., Wildling, L., Kamruzzahan, A.S., Rankl, C., Wruss, J., Hahn, C.D., Hölzl, M., Zhu, R., Kienberger, F., Blaas, D., Hinterdorfer, P., and Gruber, H.J. (2007). A new, simple method for linking of antibodies to atomic force microscopy tips. Bioconj. Chem. 18(4):1176-1184.

Eckel, R., Ros, R., Ros, A., Wilking, S.D., Sewald, N., and Anselmetti, D. (2003). Identification of binding mechanisms in single molecule-DNA complexes. Biophys. J. 85(3):1968-1973.

Edem, D.O. (2002). Palm oil: Biochemical, physiological, nutritional, hematological, and toxicological aspects: A review. Plant Foods Hum. Nutr. 57(3-4):319-341.

Engelen, L., De Wijk, R.A., Van Der Bilt, A., Prinz, J.F., Janssen, A.M., and Bosman, F. (2005). Relating particles and texture perception. Physiol. Behav. 86(1-2):111-117.

Engelen, L. and Van Der Bilt, A. (2008). Oral physiology and texture perception of semisolids. J. Texture Stud. 39(1):83-113.

Eremina, T.A., Kuznetsov, V.A., Eremin, N.N., Okhrimenko, T.M., Furmanova, N.G., Efremova, E.P., and Rak, M. (2005). On the mechanism of impurity influence on growth kinetics and surface morphology of KDP crystals. II. Experimental study of influence of bivalent and trivalent impurity ions on growth kinetics and surface morphology of KDP crystals. J. Cryst. Growth 273(3-4):586-593.

Fahy, E., Subramaniam, S., Murphy, R., Nishijima, M., Raetz, C., Shimizu, T., Spener, F., van Meer, G., Wakelam, M., and Dennis, E. (2009). Update of the LIPID MAPS comprehensive classification system for lipids. J. Lipid Res. 50(Suppl):S9-S14. 
Fennema, O.R., Powrie, W.D., and Marth, E.H. (1973). Low-Temperature Preservation of Foods and Living Matter. Marcel Dekker, New York City, New York, U.S.A.

Fernandes, V.A., Müller, A.J., and Sandoval, A.J. (2013). Thermal, structural and rheological characteristics of dark chocolate with different compositions. J. Food Eng. 116(1):97-108.

Fessas, D., Signorelli, M., and Schiraldi, A. (2005). Polymorphous transitions in cocoa butter: A quantitative DSC study. J. Therm. Anal. Calorim. 82(3):691-702.

Firestone, D. (1998). Official methods and recommended practices of the American Oil Chemists'Society. AOCS Press, Champaign, Illinois, U.S.A.

Flint, O. (1994). Food Microscopy. Bios Scientific, Oxford, U.K.

Florin, E.L., Moy, V.T., and Gaub, H.E. (1994). Adhesion forces between individual ligandreceptor pairs. Science 264(5157):415-417.

Foubert, I., Dewettinck, K., Janssen, G., and Vanrolleghem, P.A. (2006). Modelling twostep isothermal fat crystallization. J. Food Eng. 75(4):551-559.

Foubert, I., Fredrick, E., Vereecken, J., Sichien, M., and Dewettinck, K. (2008). Stop-andreturn DSC method to study fat crystallization. Thermochim. Acta 471(1-2):7-13.

Frisbie, C.D., Rozsnyai, L.F., Noy, A., Wrighton, M.S., and Lieber, C.M. (1994). Functional group imaging by chemical force microscopy. Science 265(5181):2071-2074.

Fritsche, J. and Steinhart, H. (1998). Analysis, occurrence, and physiological properties of trans fatty acids (TFA) with particular emphasis on conjugated linoleic acid isomers (CLA): A review. Eur. J. Lipid Sci. Technol. 100(6):190-210.

Galdámez, J.R., Szlachetka, K., Duda, J.L., and Ziegler, G.R. (2009). Oil migration in chocolate: A case of non-Fickian diffusion. J. Food Eng. 92(3):261-268.

Gao, Y., Sirinakis, G., and Zhang, Y. (2011). Highly anisotropic stability and folding kinetics of a single coiled coil protein under mechanical tension. J. Am. Chem. Soc. 133(32):12749-12757. 
Garside, J. (1987). General principles of crystallization. In: Food Structure and Behaviour, pp. 35-49. Blanshard, J.M.V. and Lillford, P., Eds., Academic Press, London, U.K.

Gee, P.T. (2007). Analytical characteristics of crude and refined palm oil and fractions. Eur. J. Lipid Sci. Technol. 109(4):373-379.

Ghorbel, D., Saïdi, I., Ben Slema, M., and Gharsallah, M. (2011). Adjusting flow properties of molten milk chocolate by means of emulsifiers and fat. J. Food Process Eng. 34(2):317331.

Ghosh, V., Ziegler, G.R., and Anantheswaran, R.C. (2002). Fat, moisture, and ethanol migration through chocolates and confectionary coatings. Crit. Rev. Food Sci. Nutr. 42(6):583-626.

Gibon, V., De Greyt, W., and Kellens, M. (2007). Palm oil refining. Eur. J. Lipid Sci. Technol. 109(4):315-335.

Glicerina, V., Balestra, F., Dalla Rosa, M., and Romani, S. (2013). Rheological, textural, and calorimetric modifications of dark chocolate during process. J. Food Eng. 119(1):173179.

Goh, S.H., Choo, Y.M., and Ong, S.H. (1985). Minor carotenoids of palm oil. J. Am. Oil Chem. Soc. 62(6):237-240.

Götz, J., Balzer, H., and Hinrichs, R. (2005). Characterisation of the structure and flow behaviour of model chocolate systems by means of NMR and rheology. Appl. Rheol. 15(2):98-111.

Graham, T.G.W. and Best, R.B. (2011). Force-induced change in protein unfolding mechanism: Discrete or continuous switch? J. Phys. Chem. B 115(6):1546-1561.

Gratzer, W. (2005). Terrors of the Table: The Curious History of Nutrition. Oxford University Press, New York City, New York, U.S.A.

Gresti, J., Bugaut, M., Maniongui, C., and Bezard, J. (1993). Composition of molecular species of triacylglycerols in bovine milk fat. J. Dairy Sci. 76(7):1850-1869. 
Gunstone, F.D. and Norris, F.A. (1983). Lipids in Foods: Chemistry, Biochemistry, and Technology. Pergamon Press, Oxford, U.K.

Hachiya, I., Koyano, T., and Sato, K. (1989). Seeding effects on solidification behavior of cocoa butter and dark chocolate. II. Physical properties of dark chocolate. J. Am. Oil Chem. Soc. 66(12):1763-1770.

Han, W. and Serry, F.M. (2008). Force Spectroscopy with the Atomic Force Microscope. Agilent Technologies, U.S.A.

Hartel, R.W. (1996). Applications of milk-fat fractions in confectionery products. J. Am. Oil Chem. Soc. 73(8):945-953.

Hartel, R.W. (1998). Mechanisms and kinetics of recrystallization in ice cream. In: Properties of Water in Foods: ISOPOW VI, pp. 287-319. Reid, D., Ed., Blackie, London, U.K.

Hartel, R.W. (1999). Chocolate: Fat bloom during storage. Manuf. Confect. 79:89-99.

Hartel, R.W. (2001). Crystallization in Foods. Aspen Publishers, Gaithersburg, Maryland, U.S.A.

Hartel, R.W. (2013). Advances in food crystallization. Annu. Rev. Food Sci. Technol. 4:277292.

Hayakawa, M. and DeMan, J.M. (1982). Interpretation of cone penetrometer consistency measurements of fats. J. Texture Stud. 13(2):201-210.

Haylock, S.J. and Dodds, T.M. (1999). Ingredients from milk. In: Industrial Chocolate Manufacture and Use, pp. 57-77. Beckett, S.T., Ed., Blackwell Science, Oxford, U.K.

Hernqvist, L. (1984). On the structure of triglycerides in the liquid state and fat crystallization. Fett. Wiss. Technol. 86(8):297-300.

Hernqvist, L. (1988a). Crystal structure of fats and fatty acids. In: Crystallization and Polymorphism of Fats and Fatty Acids, pp. 97-138. Garti, N. and Sato, K., Eds., Marcel Dekker, New York City, New York, U.S.A. 
Hernqvist, L. (1988b). Chocolate temper. In: Industrial Chocolate Manufacture and Use, pp. 159-171. Beckett, S.T., Ed., Blackie and Son, Glasgow, U.K.

Herrera, M.L. and Hartel, R.W. (2000). Effect of processing conditions on the crystallization kinetics of fats. J. Am. Oil Chem. Soc. 77(11):1177-1187.

Hettich, A. (1966). Experimental basis for the definition of 'proper' chocolate temper. Manuf. Confect. 46:29-36.

Himawan, C., Starov, V.M., and Stapley, A. F. (2006). Thermodynamic and kinetic aspects of fat crystallization. Adv. Colloid Interface Sci. 122(1-3):3-33.

Hinterdorfer, P., Baumgartner, W., Gruber, H.J., Schlicher, K., and Schindler, H. (1996). Detection and localization of individual antibody-antigen recognition events by atomic force microscopy. Proc. Natl. Acad. Sci. U.S.A. 93(8):3477-3481.

Hodge, S.M. and Rousseau, D. (2002). Fat bloom formation and characterization in milk chocolate observed by atomic force microscopy. J. Am. Oil Chem. Soc. 79(11):1115-1121.

Hruby, A. and Hu, F.B. (2016). Saturated fat and heart disease: The latest evidence. Lipid Technol. 28(1):7-12.

Hu, Y., Liang, J.K., Myerson, A.S., and Taylor, L.S. (2005). Crystallization monitoring by Raman spectroscopy: Simultaneous measurement of desupersaturation profile and polymorphic form in flufenamic acid systems. Ind. Eng. Chem. Res. 44(5):1233-1240.

Huyghebaert, A. and Hendrickx, H. (1971). Polymorphism of cocoa butter shown by differential scanning calorimetry. Lebensm. Wiss. Technol. 79:59-63.

Jagannathan, B., Elms, P.J., Bustamante, C., and Marqusee, S. (2012). Direct observation of a force-induced switch in the anisotropic mechanical unfolding pathway of a protein. Proc. Natl. Acad. Sci. U.S.A. 109(44):17820-17825.

James, B.J. and Smith, B.G. (2009). Surface structure and composition of fresh and bloomed chocolate analysed using $\mathrm{x}$-ray photoelectron spectroscopy, cryo-scanning electron 
microscopy, and environmental scanning electron microscopy. Lebensm. Wiss. Technol. 42(5):929-937.

James, P.F. (1982). Nucleation in glass-forming systems: A review. In: Nucleation and Crystallization in Glasses, pp. 1-48. Simmons, J.H., Uhlmann, D.R. and Beall, G.H., Eds., American Ceramic Society, Columbus, Ohio, U.S.A.

Jamieson, P. (2008). The sugarfree toolbox: Bulk ingredients and intense sweeteners. Manuf. Confect. 88:33-46.

Janshoff, A., Neitzert, M., Obersörfer, Y., and Fuchs, H. (2000). Force spectroscopy of molecular systems: Single molecule spectroscopy of polymers and biomolecules. Angew. Chem. Int. Ed. 39(18):3212-3237.

Jeffery, M.S. (1993). Key functional properties of sucrose in chocolate and sugar confectionery. Food Technol. 47:141-144.

Jewell, G.G. (1972). Some observations on bloom on chocolate. Int. Choc. Rev. 27(6):161162.

Johansson, D. and Bergenståhl, B. (1992). The influence of food emulsifiers on fat and sugar dispersions in oils. I. Adsorption, sedimentation. J. Am. Oil Chem. Soc. 69(8):705-717.

Kaiser, J.M., Gestel, A.V., and Vercauteren, J. (1998). Reduced-Fat Confectioneries Comprising Emulsifying Agent Combinations and Preparation Thereof. Patent WO 1999045790 A1.

Karupaiah, T. and Sundram, K. (2007). Effects of stereospecific positioning of fatty acids in triacylglycerol structures in native and randomized fats: A review of their nutritional implications. Nutr. Metab. 4:16.

Katzmarzyk, P.T. and Ardern, C.I. (2004). Overweight and obesity mortality trends in Canada 1985-2000. Can. J. Public Health 95(1):16-20.

Kellens, M., Gibon, V., Hendrix, M., and De Greyt, W. (2007). Palm oil fractionation. Eur. J. Lipid Sci. Technol. 109(4):336-349. 
Khan, R.S. and Rousseau, D. (2006). Hazelnut oil migration in dark chocolate: Kinetic, thermodynamic and structural considerations. Eur. J. Lipid Sci. Technol. 108(5):434-443.

Kheiri, S.A. (1987). End uses of palm oil: Human food. In: Palm Oil, pp. 71-83. Gunstone, F.D., Ed., John Wiley and Sons, Chichester, U.K.

Kilcast, D. and Clegg, S. (2002). Sensory perception of creaminess and its relationship with food structure. Food Qual. Prefer. 13(7-8):609-623.

Kleinert, J. (1961). Studies on the formation of fat bloom and methods of delaying it. Rev. Int. Choc. 16:201-219.

Kobayashi, M. (1988). Vibrational spectroscopic aspects of polymorphism and phase transition of fats and fatty acids. In: Crystallization and Polymorphism of Fats and Fatty Acids, pp. 139-187. Garti, N. and Sato, K., Eds., Marcel Dekker, New York City, New York, U.S.A.

Krog, N. (2001). Crystallization properties and lyotropic phase behaviour of food emulsifiers. In: Crystallization and Polymorphism of Fats and Fatty Acids, pp. 505-526. Garti, N. and Sato, K., Eds., Marcel Dekker, New York City, New York, U.S.A.

Kroger, M., Meister, K., and Kava, R. (2006). Low-calorie sweeteners and other sugar substitutes: A review of the safety issues. Compr. Rev. Food Sci. Food Saf. 5(2):35-47.

Kronenberger, A. (2006). Atomic force microscopy: Basics and Applications. Jacobs University Bremen, Bremen, Germany.

Krüger, C. (1999). Sugar and bulk sweetener. In: Industrial Chocolate Manufacture and Use, pp. 36-56. Beckett, S.T., Ed., Blackwell Science, Oxford, U.K.

Kuczynski, G.C. (1989). Towards the understanding of the process of sintering. In: Sintering'85, pp. 3-16. Kuczynski, G.C., Uskokovic, D.P., and Palmour III, H., Eds., Plenum Press, New York City, New York, U.S.A. 
Kulozik, U., Tolkach, A., Bulca, S., and Hinrichs, J. (2003). The role of processing and matrix design in development and control of microstructures in dairy food production: A survey. Int. Dairy J. 13(8):621-630.

L'Abbé, M.R., Stender, S., Skeaff, C.M., Ghafoorunissa, and Tevella, M. (2009). Approaches to removing trans fats from the food supply in industrialized and developing countries. Eur. J. Clin. Nutr. 63:S50-S67.

Land, T.A., Martin, T.L., and Potapenko, S. (1999). Recovery of surfaces from impurity poisoning during crystal growth. Nature 399(6735):442-445.

Larson, M.A. (1981). Secondary nucleation: An analysis. Chem. Eng. Commun. 12:161-169.

Larsson, K. (1966). Classification of glyceride crystal forms. Acta Chem. Scand. 20(8):2255-2260.

Larsson, K. (1994). Lipids: Molecular Organization, Physical Functions, and Technical Application. The Oily Press, Dundee, U.K.

Larsson, K., Quinn, P., Sato, K., and Tiberg, F. (2006). Lipids: Structure, Physical Properties, and Functionality. The Oily Press, Bridgewater, U.K.

Larumbe, A., González, H.H.L., Resnik, S.L., and Chirife, J. (1991). Moisture migration and mold growth in a composite chocolate product. Lebensm. Wiss. Technol. 24(4):307-309.

Lee, S., Heuberger, M., Rousset, P., and Spencer, N.D. (2002). Chocolate at a sliding interface. J. Food Sci. 67(7):2712-2717.

Lee, S.-W. (2004). Relaxation characteristics of poly(vinylidene fluoride) and ethylenechlorotrifluoroethylene in the transient uptake of aromatic solvents. Korean J. Chem. Eng. 21(6):1119-1125.

Lee, W.E. and Pangborn, R.M. (1986). Time-intensity: The temporal aspects of sensory perception. Food Technol. 40:71-82. 
Li, H.B., Rief, M., Oesterhelt, F., Gaub, H.E., Zhang, X., and Shen, J.C. (1999). Singlemolecule force spectroscopy on polysaccharides by AFM-nanomechanical fingerprint of alpha-(1,4)-linked polysaccharides. Chem. Phys. Lett. 305(3-4):197-201.

Liang, B. and Hartel, R.W. (2004). Effects of milk powders in milk chocolate. J. Dairy Sci. 87(1):20-31.

Litwinenko, J.W., Rojas, A.M., Gerschenson, L.N., and Marangoni, A.G. (2002). Relationship between crystallization behaviour, microstructure, and mechanical properties in a palm oil-based shortening. J. Am. Oil Chem. Soc. 79(7):647-654.

Liu, Y., Meng, Z., Zhang, F., Shan, L., and Wang, X. (2010). Influence of lipid composition, crystallization behaviour and microstructure on hardness of palm oil-based margarines. Eur. Food Res. Technol. 230(5):759-767.

Lohman, M.H. and Hartel, R.W. (1994). Effect of milk fat fractions on fat bloom in dark chocolate. J. Am. Oil Chem. Soc. 71(3):267-276.

Loisel, C., Keller, G., Lecq, G., Bourgaux, C., and Ollivon, M. (1998). Phase transitions and polymorphism of cocoa butter. J. Am. Oil Chem. Soc. 75(4):425-439.

Loisel, C., Lecq, G., Ponchel, G., Keller, G., and Ollivon, M. (1997). Fat bloom and chocolate structure studied by mercury porosimetry. J. Food Sci. 62(4):781-788.

Lonchampt, P. and Hartel, R.W. (2004). Fat bloom in chocolate and compound coatings. Eur. J. Lipid Sci. Technol. 106(4):241-274.

Lovegren, N.V., Gray, M.S., and Fuege, R.O. (1976). Effect of liquid fat on melting point and polymorphic behaviour of cocoa butter and a cocoa butter fraction. J. Am. Oil Chem. Soc. 53(3):108-112.

Maache-Rezzoug, Z., Bouvier, J.M., Allaf, K., and Patras, C. (1998). Study of mixing in connection with the rheological properties of biscuit dough and dimensional characteristics of biscuits. J. Food Eng. 35(1):43-56. 
Maleky, F. and Marangoni, A.G. (2011). Nanoscale effects on oil migration through triacylglycerol polycrystalline colloidal networks. Soft Matter 7(13):6012-6024.

Mallows, C.L. (1973). Some comments on CP. Technometrics 15(4):661-675.

Marangoni, A.G. (2005). Fat Crystal Networks. Marcel Dekker, New York City, New York, U.S.A.

Marangoni, A.G. (2012). Chocolate Compositions Containing Ethylcellulose. United States Patent 20120183651 A1.

Marangoni, A.G. and Wesdorp, L.H. (2013). Structure and Properties of Fat Crystal Networks. CRC Press, Boca Raton, Florida, U.S.A.

Marty, S., Baker, K., Dibildox-Alvarado, E., Rodrigues, J.N., and Marangoni, A.G. (2005). Monitoring and quantifying of oil migration in cocoa butter using a flatbed scanner and fluorescence light microscopy. Food Res. Int. 38(10):1189-1197.

Marty, S. and Marangoni, A.G. (2009). Effects of cocoa butter origin, tempering procedure, and structure on oil migration kinetics. Cryst. Growth Des. 9(10):4415-4423.

Mate, C.M., McClelland, G.M., Erlandsson, R., and Chiang, S. (1987). Atomic-scale friction of a tungsten tip on a graphite surface. Phys. Rev. Lett. 59(17):1942-1946.

Mathlouthi, M. and Genotelle, J. (1995). Rheological properties of sucrose solutions and suspensions. In: Sucrose, Properties and Applications, pp. 126-154. Mathlouthi, M. and Reiser, P., Eds., Blackie, Glasgow, U.K.

Mazzanti, G., Guthrie, S.E., Sirota, E.B., Marangoni, A.G., and Idziak, S.H.J. (2003). Orientation and phase transitions of fat crystals under shear. Cryst. Growth Des. 3(5):721725.

McNamara, D.J. (2010). Palm oil and health: A case of manipulated perception and misuse of science. J. Am. Coll. Nutr. 29(3 Suppl):240S-244S.

Merkel, R., Nassoy, P., Leung, A., Ritchie, K., and Evans, E. (1999). Energy landscapes of receptor-ligand bonds explored with dynamic force spectroscopy. Nature 397(6714):50-53. 
Metin, S. (1997). Crystallization Behaviour and Kinetics of Blends of Cocoa Butter and Milk Fat or Milk Fat Fractions. University of Wisconsin-Madison, Madison, Wisconsin, U.S.A. Metin, S. and Hartel, R.W. (2005). Crystallization of fats and oils. In: Bailey's Industrial Oil and Fat Products, pp. 45-76. Shahidi, F., Ed., John Wiley and Sons, Chichester, U.K.

Meyer, G. and Amer, N.M. (1988). Novel optical approach to atomic force microscopy. Appl. Phys. Lett. 53(12):1045-1047.

Middendorf, D., Juadjur, A., Bindrich, U., and Mischnick, P. (2015). AFM approach to study the function of PGPR's emulsifying properties in cocoa butter based suspensions. Food Struct. 4:16-26.

Middendorf, D., Bindrich, U., Mischnick, P., Juadjur, A., Franke, K., and Heinz, V. (2016). Atomic force microscopy study on the effect of different lecithins in cocoa butter based suspensions. Colloids Surf., A 499:60-68.

Miles, M.J. and McMaster, T.J. (1995). Scanning probe microscope in food-related systems. In: New Physicochemical Techniques for the Characterization of Complex Food Systems, pp. 69-85. Dickinson, E., Ed., Blackie, Glasgow, U.K.

Minifie, B.W. (1989). Chocolate, Cocoa, and Confectionery: Science and Technology. Chapman and Hall, London, U.K.

Minson, E. (1990). Cookie confectionery combinations. Manuf. Confect. 70:121-126.

Miquel, M.E., Carli, S., Couzens, P.J., Wille, H.-J., and Hall, L.D. (2001). Kinetics of the migration of lipids in composite chocolate measured by magnetic resonance imaging. Food Res. Int. 34(9):773-781.

Miquel, M.E. and Hall, L.D. (2002). Measurement by MRI of storage changes in commercial chocolate confectionery products. Food Res. Int. 35(10):993-998.

Mondal, K. and Murty, B.S. (2006). Prediction of maximum homogeneous nucleation temperature for crystallization of metallic glasses. J. Non-Cryst. Solids 352(50-51):52575264. 
Mongia, G. and Ziegler, G.R. (2000). The role of particle size distribution of suspended solids in defining the flow properties of milk chocolate. Int. J. Food Prop. 3(1):137-147.

Morris, V. J. (2007). Atomic force microscopy techniques for characterising food structure. In: Understanding and Controlling the Microstructure of Complex Foods, pp. 209-231. McClements, D.J., Ed., CRC Press, Boca Raton, Florida, U.S.A.

Mullin, J.W. (1993). Crystallization. Butterworth, London, U.K.

Murphy, D.J. (2007). Future prospects for oil palm in the 21st century: Biological and related challenges. Eur. J. Lipid Sci. Technol. 109(4):296-306.

Neaton, J.D. and Wentworth, D. (1992). Serum cholesterol, blood pressure, cigarette smoking, and death from coronary heart disease. Overall findings and differences by age for 316,099 white men. Multiple Risk Factor Intervention Trial Research Group. Arch. Intern. Med. 152(1):56-64.

Nightingale, L.M., Cadwallader, K.R., and Engeseth, N.J. (2012). Changes in dark chocolate volatiles during storage. J. Agric. Food Chem. 60(18):4500-4507.

Nopens, I., Foubert, I., De Graef, V., Van Laere, D., Dewettinck, K., and Vanrolleghem, P. (2008). Automated image analysis tool for migration fat bloom evaluation of chocolate coated food products. Lebensm. Wiss. Technol. 41(10):1884-1891.

Nováková, H., Čopíková, J., Maixner, J., and Maryška, M. (2002). The production of clusters in milk chocolate. Int. J. Food Sci. Technol. 37(5):485-495.

Noy, A. and Friddle, R. W. (2013). Practical single molecule force spectroscopy: How to determine fundamental thermodynamic parameters of intermolecular bonds with an atomic force microscope. Methods 60(2):142-150.

Noy, A., Frisbie, C.D., Rozsnyai, L.F., Wrighton, M.S., and Lieber, C.M. (1995). Chemical force microscopy: Exploiting chemically-modified tips to quantify adhesion, friction, and functional group distributions in molecular assemblies. J. Am. Chem. Soc. 117(30):79437951. 
Noy, A., Vezenov, D.V., and Lieber, C.M. (2008). Chemical force microscopy nanoscale probing of fundamental chemical interactions. In: Handbook of Molecular Force Spectroscopy, pp. 97-122. Noy, A., Ed., Springer Science+Business Media, New York City, New York, U.S.A.

Nusantoro, B.P. (2009). Isothermal crystallization properties of RBD-palm oil as measured by DSC and oscillatory rheometry. AGRITECH 29:177-182.

Okiy, D.A. and Oke, O.L. (1984). A study of the effects of salt and pepper on palm oil used as a frying medium. Food Chem. 14(4):251-262.

Olinger, P.M. (1994). New options for sucrose-free chocolate. Manuf. Confect. 74:77-84.

Omar, Z., Let, C.C., Seng, C.C., and Rashid, N.A. (2005). Crystallisation and rheological properties of hydrogenated palm oil and palm oil blends in relation to crystal networking. Eur. J. Lipid Sci. Technol. 107(9):634-640.

Ortiz, C. and Hadziioannou, G. (1999). Entropic elasticity of single polymer chains of poly(methacrylic acid) measured by atomic force microscopy. Macromolecules 32(3):780787.

Osim, E.E., Owu, D.U., Isong, E., and Umoh, I.B. (1992). Influence of chromic consumption of thermoxidized palm oil on platelet aggregation in the rats. Discov. Innov. 4(1):83-87.

Osim, E.E., Owu, D.U., Isong, E., and Umoh, I.B. (1994). Influence of chronic consumption of thermoxidized and fresh palm oil diets on basal metabolic rate, body weight, and morphology of tissues in rats. Discov. Innov. 6(4):389-396.

Owen, R. (2004). A Practical Guide to AFM Force Spectroscopy and Data Analysis. JPK Instruments Technical Report.

Pajin, B. and Jovanovic, O. (2005). Influence of high-melting milk fat fraction on quality and fat bloom stability of chocolate. Eur. Food Res. Technol. 220(3):389-394. 
Pérez-Martínez, D., Alvarez-Salas, C., Charó-Alonso, M., Dibildox-Alvarado, E., and ToroVazquez, J.F. (2007). The cooling rate effect on the microstructure and rheological properties of blends of cocoa butter with vegetable oils. Food Res. Int. 40(1):47-62.

Pictet, A. and Vogel, H. (1928). Saccharose B. Helv. Chim. Acta 11:901-905.

Pink, D.A., Quinn, B., Peyronel, F., and Marangoni, A.G. (2013). Edible oil structures at low and intermediate concentrations. I. Modeling, computer simulation, and predictions for x-ray scattering. J. Appl. Phys. 114(23):234901.

Purseglove, J.W. (1983). Tropical Crops Monocotyledons, Volumes 1 and 2 Combined, English Language Book Society and Longmans Group, Essex, U.K.

Quevedo, R., Valencia, E., Alvarado, F., Ronceros, B., and Bastias, J.M. (2013). Comparison of whiteness index vs. fractal Fourier in the determination of bloom chocolate using image analysis. Food Bioprocess Technol. 6(7):1878-1884.

Rak, M., Eremin, N.N., Eremina, T.A., Kuznetsov, V.A., Okhrimenko, T.M., Furmanova, N.G., and Efremova, E.P. (2005). On the mechanism of impurity influence on growth kinetics and surface morphology of KDP crystals - I: Defect centres formed by bivalent and trivalent impurity ions incorporated in KDP structure - Theoretical study. J. Cryst. Growth 273(3-4):577-585.

Randolph, A.D. and Larson, M.A. (1988). Theory of Particulate Processes. Academic Press, New York City, New York, U.S.A.

Rao, M.A. (2007). Influence of food microstructure on food rheology. In: Understand and Controlling the Microstructures of Complex Foods, pp. 411-424. McClements, D. J., Ed., CRC Press, Boca Raton, Florida, U.S.A.

Rector, D. (2000). Chocolate-controlling the flow. Benefits of polyglycerol polyricinoleic acid. Manuf. Confect. 80:63-70.

Rief, M. (1997). Reversible unfolding of individual titin immunoglobulin domains by AFM. Science 276(5315):1109-1112. 
Rief, M., Oesterhelt, F., Heymann, B., and Gaub, H.E. (1997). Single molecule force spectroscopy on polysaccharides by atomic force microscopy. Science 275(5304):12951297.

Rössner, S. (1997). Chocolate: Divine food, fattening junk or nutritious supplementation? Eur. J. Clin. Nutr. 51(6):341-345.

Rousseau, D. (2006). On the porous mesostructure of milk chocolate viewed with atomic force microscopy. Lebensm. Wiss. Technol. 39(8):852-860.

Rousseau, D. (2007). The microstructure of chocolate. In: Understanding and Controlling the Microstructure of Complex Foods, pp. 648-690. McClements, D.J., Ed., CRC Press, Boca Raton, Florida, U.S.A.

Rousseau, D., Hodge, S.M., Nickerson, M.T., and Paulson, A.T. (2005). Regulating the $\beta^{\prime} \rightarrow \beta$ polymorphic transition in food fats. J. Am. Oil Chem. Soc. 82(1):7-12.

Rousseau, D. and Marangoni, A.G. (1999). The effects of interesterification on physical and sensory attributes of butterfat and butterfat-canola oil spreads. Food Res. Int. 31(5):381-388.

Rousseau, D. and Sonwai, S. (2008). Influence of the dispersed particulate in chocolate on cocoa butter microstructure and fat crystal growth during storage. Food Biophys. 3(2):273278.

Rousseau, D., Sonwai, S., and Khan, R. (2010). Microscale surface roughening of chocolate viewed with optical profilometry. J. Am. Oil Chem. Soc. 87(10):1127-1136.

Rousset, Ph., Sellappan, P. and Daoud, P. (2002). Effect of emulsifiers on surface properties of sucrose by inverse gas chromatography. J. Chromatogr. A 969(1-2):97-101.

Russell, A.B., Cheney, P.E., and Wantling, S.D. (1999). Influence of freezing conditions on ice crystallization in ice cream. J. Food Eng. 39(2):179-191.

Saberi, A.H., Lai, O.-M., and Miskandar, M.S. (2012). Melting and solidification properties of palm-based diacylglycerol, palm kernel olein, and sunflower oil in the preparation of 
palm-based diacylglycerol-enriched soft tub margarine. Food Bioprocess Technol. 5(5):1674-1685.

Sambanthamurthi, R., Sundram, K., and Tan, Y. (2000). Chemistry and biochemistry of palm oil. Prog. Lipid Res. 39(6):507-558.

Santos, J.C.O., Santos, I.M.G., and Souza, A.G. (2005). Effect of heating and cooling on rheological parameters of edible vegetable oils. J. Food Eng. 67(4):401-405.

Sato, K. (1988). Crystallization of fats and fatty acids. In: Crystallization and Polymorphism of Fats and Fatty Acids, pp. 227-266. Garti, N. and Sato, K., Eds., Marcel Dekker, New York City, New York, U.S.A.

Sato, K. (1993). Polymorphic transformations in crystal growth. J. Phys. D: Appl. Phys. 26(8B):B77-B84.

Sato, K. (2001). Crystallization behaviour of fats and lipids: A review. Chem. Eng. Sci. 56(7):2255-2265.

Sato, K., Arishima, T., Wang, Z.H., Ojima, K., Sagi, N., and Mori, H. (1989). Polymorphism of POP and SOS. I. Occurrence and polymorphic transformation. J. Am. Oil Chem. Soc. 66(5):664-674.

Savage, C.M. and Dimick, P.S. (1995). Influence of phospholipids during crystallization of hard and soft cocoa butters. Manuf. Confect. 75:127-132.

Schantz, B., Linke, L., and Rohm, H. (2003). Effects of different emulsifiers on rheological and physical properties of chocolate. In: Proceedings of the 3rd International Symposium on Food Rheology and Structure, pp. 329-333. Fischer, P., Marti, I., and Windhab, E.J., Eds., ETH - Laboratory of Food Process Engineering, Zurich, Switzerland.

Schantz, B. and Rohm, H. (2005). Influence of lecithin-PGPR blends on the rheological properties of chocolate. Lebensm. Wiss. Technol. 38(1):41-45.

Schenk, H. and Peschar, R. (2004). Understanding the structure of chocolate. Radiat. Phys. Chem. 71(3-4):829-835. 
Schlichter-Aronhime, J. and Garti, N. (1988). Solidification and polymorphism in cocoa butter and the blooming problems. In: Crystallization and Polymorphism of Fats and Fatty Acids, pp. 363-393. Garti, N. and Sato, K., Eds., Marcel Dekker, New York City, New York, U.S.A.

Schoukens, G. and De Clerck, K. (2005). Thermal analysis and Raman spectroscopic studies of crystallization in poly(ethylene 2,6-naphthalate). Polymer 46(3):845-857.

Seguine, E.S. (1991). Tempering: The inside story. Manuf. Confect. 5:117-125.

Sen, C.K., Khanna, S., and Roy, S. (2007). Tocotrienols in health and disease: The other half of the natural vitamin E family. Mol. Aspects Med. 28(5-6):692-728.

Seo, Y. and Jhe, W. (2008). Atomic force microscopy and spectroscopy. Rep. Prog. Phys. 71(1):1-23.

Servais, C., Jones, R., and Roberts, I. (2002). The influence of particle size distribution on the processing of food. J. Food Eng. 51(3):201-208.

Servais, C., Ranc, H., and Roberts, I.D. (2004). Determination of chocolate viscosity. $J$. Texture Stud. 34(5-6):467-497.

Shah, A.B., Jones, G.P., and Vasiljevic, T. (2010). Sucrose-free chocolate sweetened with Stevia rebaudiana extract and containing different bulking agents: Effects on physicochemical and sensory properties. Int. J. Food Sci. Technol. 45(7):1426-1435.

Sherazi, S.T.H., Kandhro, A., Mahesar, S.A., Bhanger, M.I., Talpur, M.Y., and Arain, S. (2009). Application of transmission FT-IR spectroscopy for the trans fat determination in the industrially processed edible oils. Food Chem. 114(1):323-327.

Siew, W.L. and Ng, W.L. (1995). Partition coefficient of diglycerides in crystallization of palm oil. J. Am. Oil Chem. Soc. 72(5):591-595.

Siew, W.-L., and Ng, W.-L. (1996a). Effect of digylcerides on the crystallisation of palm oleins. J. Sci. Food Agric. 71(4):496-500. 
Siew, W.L. and Ng, W.L. (1996b). Characterisation of crystals in palm olein. J. Sci. Food Agric. 70(2):212-216.

Siew, W.L. and Ng, W.L. (1999). Influence of diglycerides on crystallisation of palm oil. $J$. Sci. Food Agric. 79(5):722-726.

Simecek-Beatty, D., Lehr, W.J., and Lankford, J.F. (2001). Leaking Tank Experiments With Orimulsion ${ }^{\mathrm{TM}}$ and Canola Oil. In: NOAA Technical Memorandum NOS OR\&R 6. NOAA, Seattle, Washington, U.S.A.

Skeaff, C.M. (2009). Feasibility of recommending certain replacement or alternative fats. Eur. J. Clin. Nutr. 63(Suppl 2):S34-S49.

Smith, K.W. (2001). Crystallization of palm oil and its fractions. In: Crystallization Processes in Fats and Lipid Systems, pp. 357-381. Garti, N. and Sato, K., Eds., Marcel Dekker, New York City, New York, U.S.A.

Smith, K.W., Bhaggan, K., Talbot, G., and Van Malssen, K.F. (2011). Crystallization of fats: Influence of minor components and additives. J. Am. Oil Chem. Soc. 88(8):1085-1101.

Smith, K.W., Cain, F.W., and Talbot, G. (2007). Effect of nut oil migration on polymorphic transformation in a model system. Food Chem. 102(3):656-663.

Smith, P.R. and Dahlman, A. (2005). The use of atomic force microscopy to measure the formation and development of chocolate bloom in pralines. J. Am. Oil Chem. Soc. 82(3):165-168.

Snedecor, G.W. and Cochran, W.G. (1989). Statistical Methods. Iowa State University Press, Ames, USA.

Sokmen, A. and Gunes, G. (2006). Influence of some bulk sweeteners on rheological properties of chocolate. Lebensm. Wiss. Technol. 39(10):1053-1058.

Sonoda, T., Takata, Y., Ueno, S., and Sato, K. (2004). DSC and synchrotron-radiation x-ray diffraction studies on crystallization and polymorphic behavior of palm stearin in bulk and oil-in-water emulsion states. J. Am. Oil Chem. Soc. 81(4):365-373. 
Sonwai, S. and Rousseau, D. (2008). Fat crystal growth and microstructural evolution in milk chocolate. Cryst. Growth Des. 8(9):3165-3174.

Sonwai, S. and Rousseau, D. (2010). Controlling fat bloom formation in chocolate: Impact of milk fat on microstructure and fat phase crystallisation. Food Chem. 119(1):286-297.

Spearing, N. (2007). The Effect of Fat on the Texture of Milk Chocolate. University of Nottingham, Nottingham, U.K.

Stanley, D.W., Aguilera, J.M., Baker, K.W., and Jackman, R.L. (1998). Structure/property relationships of foods affected by processing and storage. In: Phase/State Transitions in Foods, pp. 1-56. Rao, M. A. and Hartel, R. W., Eds., Marcel Dekker, New York City, New York, U.S.A.

Stanley, J.C. (2008). The nutritional reputation of palm oil. Lipid Technol. 20(5):112-114.

Stapley, A.G.F., Tewkesbury, H., and Fryer, P.J. (1999). The effects of shear and temperature history on the crystallization of chocolate. J. Am. Oil Chem. Soc. 76(6):677685.

Statistics Canada. (2012). Canada Health Measures Survey - Body composition of Canadian adults, 2009-2011. Retrieved February 12, 2018, from http://www.statcan.gc.ca/pub/82-625-x/2012001/article/11708-eng.htm

Subramaniam, P.J. (2000). Confectionery products. In: The Stability and Shelf Life of Food, pp. 221-248. Kilcast, D. and Subramaniam, P.J., Eds., Woodhead Publishing, Cambridge, U.K.

Sundram, K. and Top, A.G.M. (1994). Vitamin E from palm oil: Its extraction and nutritional properties. Palmas 15(1):77-82.

Svanberg, L., Ahrné, L., Lorén, N., and Windhab, E. (2011a). Effect of sugar, cocoa particles and lecithin on cocoa butter crystallisation in seeded and non-seeded chocolate model systems. J. Food Eng. 104(1):70-80. 
Svanberg, L., Ahrné, L., Lorén, N., and Windhab, E. (2011b). Effect of pre-crystallization process and solid particle addition on microstructure in chocolate model systems. Food Res. Int. 44(5):1339-1350.

Svanberg, L., Ahrné, L., Lorén, N., and Windhab, E. (2013). Impact of pre-crystallization process on structure and product properties in dark chocolate. J. Food Eng. 114(1):90-98.

Svanberg, L., Lorén, N., and Ahrné, L. (2012). Chocolate swelling during storage caused by fat or moisture migration. J. Food Sci. 77(11):E328-E334.

Talbot, G. (1994). Chocolate temper. In: Industrial Chocolate Manufacture and Use, pp. 156-166. Beckett, S.T., Ed., Blackie, Oxford, U.K.

Talbot, G. (1999). Vegetable fats. In: Industrial Chocolate Manufacture and Use, pp. 307322. Beckett, S.T., Ed., Blackie, Oxford, U.K.

Tang, D. and Marangoni, A. G. (2007). Structure and function of fat crystals and their role in microstructure formation in complex foods. In: Understanding and Controlling the Microstructure of Complex Foods, pp. 67-88. McClements, D.J., Ed., CRC Press, Boca Raton, Florida, U.S.A.

Tang, J., Ebner, A., Ilk, N., Lichtblau, H., Huber, C., Zhu, R., Pum, D., Leitner, M., Pastushenko, V., Gruber, H.J., Sleytr, U.B., and Hinterdorfer, P. (2008). High-affinity tags fused to s-layer proteins probed by atomic force microscopy. Langmuir 24(4):1324-1329.

Tarabukina, E., Jego, F., Haudin, J.M., Navard, P., and Peuvrel-Disdier, E. (2009). Effect of shear on the rheology and crystallization of palm oil. J. Food Sci. 74(8):405-416.

Tarrago-Trani, M.T., Phillips, K.M., Lemar, L.E., and Holden, J.M. (2006). New and existing oils and fats used in products with reduced trans-fatty acid content. J. Am. Diet. Assoc. 106(6):867-880.

Tavare, N.S. (1995). Industrial Crystallization: Process Simulation Analysis and Design. Plenum Press, New York City, New York, U.S.A. 
Tietz, R.A. and Hartel, R.W. (2000). Effects of minor lipids on crystallization of milk fatcocoa butter blends and bloom formation in chocolate. J. Am. Oil Chem. Soc. 77(7):763771.

Timms, R. E. (1984). Phase behaviour of fats and their mixtures. Prog. Lipid Res. 23(1):138.

Timms, R. E. (1991). Crystallisation of fats. Chemistry and Industry 10:342-345.

Timms, R.E. (2003). Confectionery Fat Handbook: Properties, Production, and Applications. The Oily Press, London, U.K.

Toro-Vazquez, J., Herrera-Coronado, V., Dibildox-Alvarado, E., Charo-Alonso, M., and Gomez-Aldapa, C. (2002). Induction time of crystallization in vegetable oils, comparative measurements by differential scanning calorimetry and diffusive light scattering. J. Food Sci. 67(3):1057-1065.

Tscheuschner, H.D. (1994). Rheological and processing properties of fluid chocolate. Appl. Rheol. 94:83-88.

Tscheuschner, H.D. and Wünsche, D. (1979). Rheological properties of chocolate masses and the influence of some factors. In: Food Texture and Rheology, pp. 355-368. Sherman, P., Ed., Academic Press, New York City, New York, U.S.A.

Tshiamala, I.T. (2013). Influence of a Commercial Monoglyceride on the Microstructural Development of Palm Oil. Ghent University, Ghent, Belgium.

Turnbull, D. and Fisher, J.C. (1949). Rate of nucleation in condensed systems. J. Chem. Phys. 17:71-73.

Vaeck, S.V. (1960). Cocoa butter and fat bloom. Manuf. Confect. 40:35-46 and 71-74.

Van Den Tempel, M. (1979). Rheology of concentrated suspensions. J. Colloid Interface Sci. 71(1):18-20. 
Van Der Vaart, K., Depypere, F., De Graef, V., Schall, P., Fall, A., Bonn, D., and Dewettinck, K. (2013). Dark chocolate's compositional effects revealed by oscillatory rheology. Eur. Food Res. Technol. 236(6):931-942.

Van Langevelde, A.J., Driessen, R., Molleman, W., Peschar, R., and Schenk, H. (2001a). Cocoa butter long spacings and the memory effect. J. Am. Oil Chem. Soc. 78(9):911-918.

Van Langevelde, A.J., Van Malssen, K., Peschar, R., and Schenk, H. (2001b). Effect of temperature on re-crystallization behaviour of cocoa butter. J. Am. Oil Chem. Soc. 78(9):919-925.

Van Malssen, K.F., Peschar, R., and Schenk, H. (1994). Geometrical aspects of real-time powder diffraction using a normal generator and a linear diode-array detector. J. Appl. Crystallogr. 27(3):302-315.

Van Malssen, K.F., Van Langevelde, A.J., Peschar, R., and Schenk, H. (1999). Phase behaviour and extended phase scheme of static cocoa butter investigated with real-time xray powder diffraction. J. Am. Oil Chem. Soc. 76(6):669-676.

Vernier, F.C. (1998). Influence of Emulsifiers on the Rheology of Chocolate and Suspensions of Cocoa or Sugar Particles in Oil. University of Reading, Reading, U.K.

Verstringe, S., Danthine, S., Blecker, C., Depypere, F., and Dewettinck, K. (2012). Influence of monopalmitin on the isothermal crystallization mechanism of palm oil. Food Res. Int. 51(1):344-353.

Vivar-Vera, G., Torrestiana-Sanchez, B., Monroy-Rivera, J.A., and Brito-De La Fuente, E. (2008). Conching: Rheological and structural changes of chocolate mass. Dtsch. Lebensmitt. Rundsch. 104(8):376-382.

Voon, P.T., Ng, T.K.W., Lee, V.K.M., and Nesaretnam, K. (2011). Diets high in palmitic acid (16:0), lauric and myristic acids $(12: 0+14: 0)$, or oleic acid (18:1) do not alter postprandial or fasting plasma homocysteine and inflammatory markers in healthy Malaysian adults. Am. J. Clin. Nutr. 94(6):1451-1457. 
Walker, J.H. (2009). Bulk chocolate handling. In: Industrial Chocolate Manufacture and Use, pp. 169-188. Beckett, S.T., Ed., Blackwell Publishing, Chichester, U.K.

Walstra, P. (2003). Physical Chemistry of Foods. Marcel Dekker, New York City, New York, U.S.A.

Walstra, P., Kloek, W., and van Vliet, T. (2001). Fat crystal networks. In: Crystallization Processes in Fats and Lipid Systems, pp. 289-328. Garti, N. and Sato, K., Eds., Marcel Dekker, New York City, New York, U.S.A.

Walter, P. and Cornillon, P. (2002). Lipid in two-phase chocolate systems investigated by NMR and DSC. Food Res. Int. 35(8):761-767.

Walton, A.G. (1969). Nucleation in liquids and solutions. In: Nucleation, pp. 225-308. Zettlemoyer, A.C., Ed., Marcel Dekker, New York City, New York, U.S.A.

Wang, C., Shi, W.Q., Zhang, W.K., Zhang, X., Katsumoto, Y., and Ozaki, Y. (2002). Force spectroscopy study on poly(acrylamide) derivatives: Effects of substitutes and buffers on single-chain elasticity. Nano Lett. 2(10):1169-1172.

Wang, F., Liu, Y., Shan, L., Jin, Q., Wang, X., and Li, L. (2010). Blooming in cocoa butter substitutes based compound chocolate: Investigations on composition, morphology and melting behavior. J. Am. Oil Chem. Soc. 87(10):1137-1143.

Ward, I.M. and Sweeney, J. (2012). Mechanical Properties of Solid Polymers, John Wiley and Sons, Chichester, U.K.

West, R. and Rousseau, D. (2016). Crystallization and rheology of palm oil in the presence of sugar. Food Res. Int. 85:224-234.

West, R. and Rousseau, D. (2018). The role of non-fat ingredients on confectionery fat crystallization. Crit. Rev. Food Sci. Nutr. 58(11):1917-1936.

West, R. and Rousseau, D. (2017). Modelling sugar, processing, and storage effects on palm oil crystallization and rheology. LWT - Food Sci. Technol. 83:201-212. 
Wille, R.L. and Lutton, E.S. (1966). Polymorphism of cocoa butter. J. Am. Oil Chem. Soc. 43(8):491-496.

Windhab, E.J. (1999). New developments in crystallization processing. J. Therm. Anal. Calorim. 57(1):171-180.

Yoshikawa, S., Kida, H., and Sato, K. (2014). Promotional effects of new types of additives on fat crystallization. J. Oleo Sci. 63(4):333-345.

Yucel, U. and Coupland, J.N. (2011). Ultrasonic attenuation measurements of the mixing, agglomeration, and sedimentation of sucrose crystals suspended in oil. J. Am. Oil Chem. Soc. 88(1):33-38.

Zarić, D.B., Pajin, B.S., Lončarević, I.S., Šoronja Simović, D.M., and Šereš, Z.I. (2012). The impact of the manufacturing process on the hardness and sensory properties of milk chocolate. Acta Period. Technol. 43:139-148.

Zeng, Y. (2000). Seed and Shear Crystallization of Chocolate. ETH Zurich, Zurich, Switzerland.

Zettlemoyer, A.C., Ed. (1969). Nucleation. Marcel Dekker, New York City, New York, U.S.A.

Zhang, Q. and Marszalek, P. E. (2006). Identification of sugar isomers by single-molecule force spectroscopy. J. Am. Chem. Soc. 128(17):5596-5597.

Zhang, W. and Zhang, Y. (2003). Single molecule mechanochemistry of macromolecules. Prog. Polym. Sci. 28(8):1271-1295.

Zheng, P., Chou, C.-C., Guo, Y., Wang, Y., and Li, H. (2013). Single molecule force spectroscopy reveals the molecular mechanical anisotropy of the $\mathrm{FeS}_{4}$ metal center in rubredoxin. J. Am. Chem. Soc. 135(47):17783-17792.

Ziegleder, G. (1997). Fat bloom and migration. Manuf. Confect. 77:43-44. 
Ziegleder, G. (2009). Flavour development in cocoa and chocolate. In: Industrial Chocolate Manufacture and Use, pp. 169-188. Beckett, S.T., Ed., Blackwell Publishing, Chichester, U.K.

Ziegleder, G., Moser, C. and Geier Greguska, J. (1996). Kinetics of fat migration within chocolate products. Part 1. Principles and analytics. Eur. J. Lipid Sci. Technol. 98(6):196199.

Ziegleder, G. and Schwingshandl, I. (1998). Kinetics of fat migration within chocolate products. Part 3: Fat bloom. Eur. J. Lipid Sci. Technol. 100(9):411-415.

Ziegler, G.R., Mongia, G., and Hollender, R. (2001). The role of particle size distribution of suspended solids in defining the sensory properties of milk chocolate. Int. J. Food Prop. 4(2):353-370.

Zumbé, A. and Brinkworth, R. (1993). Product and Process for Producing Milk Chocolate. United States Patent US 5238698 A. 